\title{
Improving the outcome of liver surgery : towards scarless treatment of liver lesions
}

Citation for published version (APA):

Stoot, J. H. M. B. (2012). Improving the outcome of liver surgery : towards scarless treatment of liver lesions. [Doctoral Thesis, Maastricht University]. Datawyse / Universitaire Pers Maastricht. https://doi.org/10.26481/dis.20121221js

Document status and date:

Published: 01/01/2012

DOI:

10.26481/dis.20121221js

Document Version:

Publisher's PDF, also known as Version of record

\section{Please check the document version of this publication:}

- A submitted manuscript is the version of the article upon submission and before peer-review. There can be important differences between the submitted version and the official published version of record.

People interested in the research are advised to contact the author for the final version of the publication, or visit the DOI to the publisher's website.

- The final author version and the galley proof are versions of the publication after peer review.

- The final published version features the final layout of the paper including the volume, issue and page numbers.

Link to publication

\footnotetext{
General rights rights.

- You may freely distribute the URL identifying the publication in the public portal. please follow below link for the End User Agreement:

www.umlib.nl/taverne-license

Take down policy

If you believe that this document breaches copyright please contact us at:

repository@maastrichtuniversity.nl

providing details and we will investigate your claim.
}

Copyright and moral rights for the publications made accessible in the public portal are retained by the authors and/or other copyright owners and it is a condition of accessing publications that users recognise and abide by the legal requirements associated with these

- Users may download and print one copy of any publication from the public portal for the purpose of private study or research.

- You may not further distribute the material or use it for any profit-making activity or commercial gain

If the publication is distributed under the terms of Article $25 \mathrm{fa}$ of the Dutch Copyright Act, indicated by the "Taverne" license above, 


\section{Improving the outcome of liver surgery}

Towards scarless treatment of liver lesions 
ISBN 9789461591944

Image on cover: 'The liver', Artwork by Philip (9), Anthony (7) and Emilie (4)

Production: Datawyse | Universitaire Pers Maastricht

Publication of this thesis was financially supported by: Covidien B.V., Johnson \& Johnson Medical B.V., Krijnen Medical Innovations B.V., Olympus Nederland B.V., Takeda Nederland B.V., Pfizer B.V., B.Braun Medical B.V.

(C) 2012 J.H.M.B. Stoot

All rights reserved. No part of this thesis may be reproduced or transmitted in any form, by any means, electronic or mechanical, without the prior permission of the author, or where appropriate, of the publisher of the articles. 


\title{
Improving the outcome of liver surgery Towards scarless treatment of liver lesions
}

\author{
PROEFSCHRIFT \\ ter verkrijging van de graad van doctor aan de Universiteit Maastricht, \\ op gezag van Rector Magnificus prof. dr. L.L.G. Soete, \\ volgens het besluit van het College van Decanen,
} in het openbaar te verdedigen op vrijdag 21 december 2012 om 14:00 uur

door

Jan Henri Marie Basile Stoot

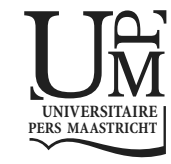




\section{PROMOTOR}

Prof. dr. C.H.C. Dejong

\section{BEOORDELINGSCOMMISSIE}

Prof. dr. C.A. Bruggeman (voorzitter)

Prof. dr. I.H.M. Borel Rinkes, Universitair Medisch Centrum Utrecht

Prof. dr. A.P. de Bruïne

Prof. dr. O.J. Garden, Royal Infirmary of Edinburgh, University of Edinburgh, Edinburgh, UK

Prof. dr. M.W. de Haan 
Aan mijn ouders Voor Patricia, Philip, Anthony \& Emilie 



\section{Contents}

$\begin{array}{lll}\text { Part } 1 \text { General introduction } & 11\end{array}$

$\begin{array}{lll}1.1 & \text { Introduction } & 13\end{array}$

$\begin{array}{lll}1.2 & \text { Liver surgery } & 17\end{array}$

$\begin{array}{lll}1.3 & \text { Malignant lesions } & 18\end{array}$

$\begin{array}{lll}1.4 & \text { Benign liver lesions } & 19\end{array}$

1.5 Benign cystic liver lesions: hydatid disease 22

1.6 Minimally invasive surgery 26

1.7 Enhanced Recovery After Surgery (ERAS) or fast-track liver surgery 28

$\begin{array}{lll}1.8 & \text { Future perspectives } & 31\end{array}$

1.9 Outline of this thesis 34

Part 2 Surgical management of benign cystic liver lesions: hydatid 45 disease

$\begin{array}{ll}\text { Introduction } & 47\end{array}$

2 The first locally-acquired human infection of Echinococcus 49 multilocularis in the Netherlands

J Clin Microbiol. 2012;50(5):1818-20

3 Over 25 years of surgical treatment of hydatid cysts in a nonendemic area using the 'frozen seal' method World J Surg. 2010;34:106-13

4 Hepatic echinococcosis in a non-endemic region: Trends in incidence, diagnosis and treatment submitted

Part 3 Surgical management of benign solid liver lesions: hepatocellular adenomas

Introduction

5 Life threatening haemorrhage from adenomas in the liver during pregnancy

Dig Surg 2006; 23:155 
6 Necrosis of a large hepatic tumour after haemorrhage and subsequent selective arterial embolisation

World J Gastroenterol 2006; 12: 6059-61

$7 \quad$ Life saving therapy for haemorrhaging liver adenomas using selective arterial embolisation BJS 2007; 94:1249-53 (Schoemaker Award 2007)

8 Malignant transformation of hepatocellular adenomas into hepatocellular carcinomas: a systematic review including more than 1600 adenoma cases

HPB 2010;12:509-22

Part 4 Reducing the impact of surgical trauma: ERAS and laparoscopic 143 surgery

Introduction

9 The introduction of a laparoscopic liver surgery programme: A cost analysis of initial experience in a university hospital Scand J Surg 2012;101:32-7

10 The effect of a multimodal 'fast track' programme on outcomes in laparoscopic liver surgery: A multicentre pilot study HPB 2009; 11: 140-44

11 Laparoscopic liver resection in the Netherlands: How far are we? Dig Surg, 2012;29(1):70-8

12 A survey in the HPB community on ways to enhance patient 189 recovery $H B P$ (in press)

Part 5 Future perspectives

Introduction

13 Prospective volumetric assessment of the liver on surgeon's a personal computer by nonradiologists prior to partial hepatectomy World J Surg 2011;35:386-92

14 Selective arterial embolisation versus resection of hepatocellular adenomas (SILVER-trial):

Study protocol for a randomised controlled trial 
15 Open versus laparoscopic left lateral hepatic sectionectomy within an Enhanced Recovery After Surgery (ERAS ${ }^{\circledR}$ ) programme

(ORANGE II Trial): Study protocol for a randomised controlled trial Trials 2012 May 6;13:54

Part 6 General discussion

16 General discussion and summary 269

17 Summary (in Dutch)

Acknowledgements

Curriculum Vitae

List of publications

295 



\section{Chapter 1}

\section{General Introduction}

J.H.M.B. Stoot, R.J.S. Coelen, J. van Vugt, C.H.C. Dejong

Adapted from 'Advances in Hepatic Surgery' in: Hepatic Surgery (in press) 


\section{ABSTRACT}

Resection of part of the liver is nowadays performed for malignant and benign liver tumours. Before the 1980's this type of surgery was still associated with a high morbidity and operative mortality. With better knowledge of the segmental anatomy, it was shown that parenchyma-sparing segmental resections were equally effective as classic lobar resections. Also, anaesthetic care and liver transection techniques were improved over time. Current data show mortality rates less than $5 \%$ in high volume centres. The 5 -year survival rates have increased from $20 \%$ in the beginning to as high as $67 \%$ in selected patients.

The development of the laparoscopic approach resulted in longer operation time in some studies. Comparing the laparoscopic technique with open liver resections, it proved to be superior with regard to blood loss, portal clamp time, overall and liver specific complications, length of stay. No difference was found for oncologic outcomes between the laparoscopic and open surgical techniques. Furthermore, multimodal enhanced recovery programmes proved to be beneficial in liver surgery with significant reduction of length of hospital stay.

The perioperative outcome after liver resection has improved over time, due to increased knowledge of liver anatomy and function, improvement of operating techniques and advances in perioperative care.

In this chapter, the improvements of liver surgery are described in historical perspective. 


\subsection{INTRODUCTION}

Hepatic resection is a commonly performed procedure for a variety of malignant and benign hepatic tumours. ${ }^{1,2}$ Historically, liver resection, irrespective of the indication, was associated with a high morbidity and mortality. ${ }^{2-4}$ During the last decades however, perioperative outcome after hepatic resection has improved, due to increased knowledge of liver anatomy and function, improvement of operating techniques and advances in anaesthesia and post-operative care. ${ }^{1,3,4}$

Hepatic resectional surgery is possible since the liver has the ability to regenerate. Although it is doubtful whether the ancient Greeks already appreciated this unique quality of the liver, it was first described in the myth of Prometheus

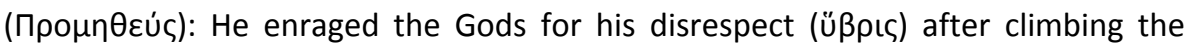
Olympus Mountain and stealing the torch in order to give fire to the humans. He was punished by Zeus, and chained to a rock in the Kaukasus Mountains. Every couple of days, an eagle came and ate part of his liver. As the liver regenerated every time, the eagle returned again and again to eat the liver and thereby torture poor Prometheus (Figure 1). With this ancient knowledge it was considered possible to take parts of the liver, as this organ has enough capacity to work with a smaller part and can regenerate.

Apart from the eagle, no human dared to remove a part of the liver. In the Ancient period of the Assyrian and Babylonian cultures of $2000-3000$ BC the liver played an important role to predict the future by reading the surface of sacrificed animals. ${ }^{5}$ This was also common in the Etruscan society, where the haruspices predicted the future from sheep livers. Hippocrates (460-377 BD), one of the founding fathers of ancient medicine, produced not only an oath with ethical rules, which is still used in modern times for all doctors. His careful observations also led to the recommendation to incise and drain abscesses of the liver with a knife. ${ }^{5}$ Celsus documented the treatment of exposed liver in war wounds. Although he was not a physician, he described his observations in the first century AD from the Alexandrian school led by Herophilus of Chalcedon and Erisastratus of Chios. ${ }^{5}$ In the same era, the Greek Galen became one of the emperors physicians in Rome and wrote reports about the dissection of many species of animals, including primates. He described the central role of the liver in absorption and digestion and his work remained of great importance for the coming centuries. ${ }^{5}$ In the centuries thereafter many reports were produced describing the treatment of war or trauma wounds.

Glisson performed extensive investigations of the vascular anatomy in $1654 .{ }^{6} \mathrm{It}$ took more than two centuries before his work was rediscovered and further clarified by Rex (1888) in Germany and Cantlie (1897) in England., These contributions led to the division of the liver in a left and right lobe. ${ }^{5}$ 


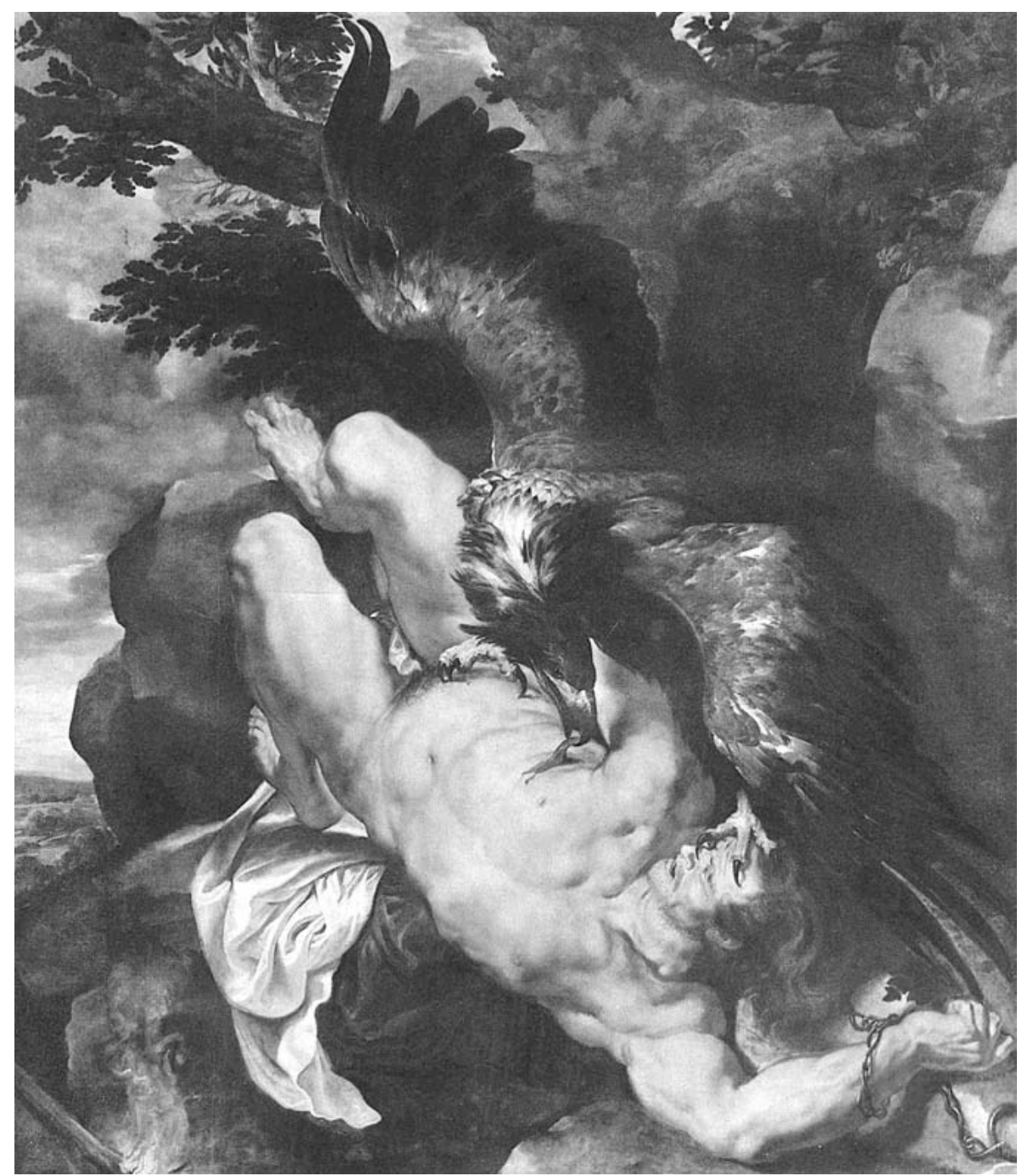

Figure 1. Prometheus chained $(243$ x 210 cm), Peter Paul Rubens, ca. 1611-1618, Philadelphia, Philadelphia Museum of Art

\section{History of liver surgery}

It still took 17 centuries before Hildanus successfully performed the first partial liver resection for trauma. ${ }^{8}$ The introduction of ether anaesthesia (1846) and the growing knowledge of antisepsis (1867) made successful elective abdominal operations possible (table 1$)^{5}$ Langenbuch was the first to perform a successful elective liver resection in 1887 and Wendel did the first hemihepatectomy in 1911. ${ }^{8}$ The principles of liver haemostasis and regeneration were determined in the period 
$1880-1900 .^{8}$ The knowledge of the principle of inflow and outflow of the liver and vascular control was one of the major advancements. Before that, wedge resections and mattress sutures were mostly used. This insight of inflow and outflow reduction was marked by the publication of J. Hogart Pringle of Glasgow, Scotland. ${ }^{9}$ He described the idea of digital control of the hilar ligament to reduce liver haemorrhage. In his famous report (1908) on liver haemorrhage after trauma, eight patients were included: three died before the operation, one refused the operation and all four operated patients died: two died during the operation, two shortly thereafter. ${ }^{5,9}$ However, his idea of digital vascular control of the hilum was more successful in the laboratory setting, where he operated three rabbits with better results, which led to his publication. Now, more than a century later, the 'Pringle manoeuvre' or 'Pringle's pinch' is still used worldwide in hepatic resectional surgery and taught all young surgeons to control haemorrhage of the liver.

Table 1. Advances in the beginning of surgery ${ }^{5}$

\begin{tabular}{lll}
\hline 1846 & Introduction of Ether anaesthesia & \\
1863 & Bacterial fermentation of wine & Pasteur \\
1867 & Antisepsis & Lister \\
1870 & First successful excision of section of the liver & Bruns \\
1880 & Discovery of Streptococci, staphylococci and pneumococci & Pasteur \\
1881 & First successful gastrectomy & Billroth \\
1882 & First successful cholecystectomy & Langenbuch \\
1883 & First human colon anastomosis & Billroth and Senn \\
1884 & Pancreas excised for cancer & Billroth \\
1886 & Report on appendicitis & Fitz \\
& Introduction of sterilisation by steam & Von Bergmann \\
1887 & First elective liver resection for adenoma & Lius \\
1887 & Successful packing of stabwound of liver & Langenbuch \\
1888 & First successful laparotomy for traumatic liver injury & Burckhardt \\
\hline
\end{tabular}

Liver surgery became gradually more popular as a better understanding of anatomic segments was established after the work of Couinaud. ${ }^{10}$ The classic morphological (outside) anatomy with two main lobes (left and right) was extended by the internal hepatic anatomy with several independent functional segments (figure 2). Each hepatic segment consists of liver parenchyma with an efferent hepatic vein branch and a portal triad; a hepatic artery branch, an afferent portal vein, and an efferent bile duct. The classic right lobe consists of four segments, the left lobe consists of three segments and the caudate lobe is segment 1. 


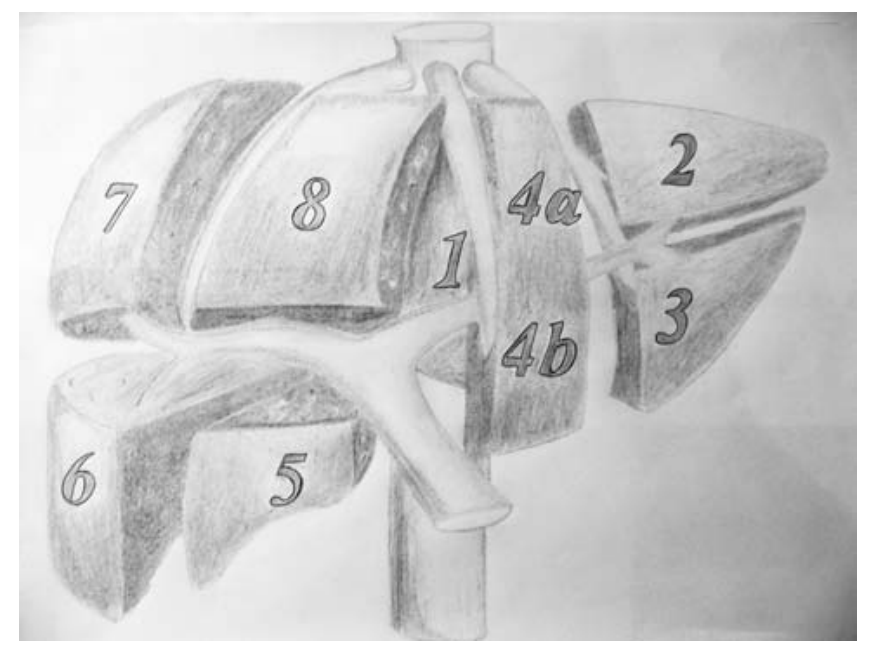

Figure 2. The anatomy of the liver with separate segments following Couinaud's classification. In this drawing only major venous vessels are displayed (portal vein, caval vein and hepatic veins).

With knowledge of the segmental anatomy of the liver, a safe transection plane could be chosen for resection without excessive blood loss and without necrosis of remnant liver. This specific anatomy of independent functional segments made it possible to resect parts of the liver without compromising the hepatic function of remnant segments. Moreover, as already described by the myth of Prometheus, the liver has regeneration capacity in contrast to other human organs. In other words after partial resections, the liver can recover its mass and function. The term ' function of the liver' is actually a collective term for a range of functions including amongst others ammonia detoxification and urea synthesis, bile synthesis and secretion, protein synthesis, gluconeogenesis and clearance or detoxification of drugs, bacterial toxins and bacteria. ${ }^{11}$ As the liver is the main detoxifying organ in humans, adaptation of its function is crucial to survive. Regeneration however, takes time. After liver surgery with a reduction of the hepatic cell mass, a 'survival programme' may start for vital liver functions. ${ }^{12}$ Some of these functions are increased rapidly in the remnant liver after resection. ${ }^{13}$ In the light of major hepatic resections, it is conceivable that too little functional liver remnant may lead to liver failure, a lethal complication of liver surgery. 


\subsection{LIVER SURGERY}

Hepatobiliary surgery incorporates a wide range of indications for surgical treatment of the liver, varying from biopsy and resection to liver transplantation. The most important indications for surgical treatment are liver lesions: these comprise a wide range of both benign and malignant lesions, which can be either primary tumours (hepatocellular carcinoma) or secondary tumours (i.e. metastases). Also, some infectious diseases of the liver (such as echinococcosis) may be an indication for surgery. Irreversible liver dysfunction caused by acute or chronic liver diseases, may be an indication for transplantation of the liver. Other benign diseases of the liver such as symptomatic simple cysts and Polycystic Liver Disease (PCLD) may also warrant surgical treatment. Other reasons for surgery of the liver may be after severe injury or trauma of the liver. The latter indications and transplantation are beyond the scope of this chapter. Since hepatic lesions form the main surgical indication for hepatic diseases, we will focus on the resectional type of liver surgery.

\section{History of liver surgery for malignant lesions}

The report of the first anatomical right hepatectomy for cancer by Lortat-Jacob in 1952 marked a new era in liver surgery. ${ }^{14}$ In the beginning, however, blood loss and mortality were considerable. A multicentre analysis in 1977 of more than 600 hepatic resections for various indications showed an operative mortality of $13 \%$, which rose to $20 \%$ for major resections. ${ }^{15}$ Despite this, pioneers in liver surgery continued the quest for improving this challenging field of expertise and gradually mortality decreased to $5.6 \%{ }^{16}$ The 5 year survival rates have increased from $20 \%$ in the beginning ${ }^{16,17}$ to as high as $67 \%$ in selected patients. ${ }^{18}$ Earlier developments in liver surgery have been marked by major contributions of Starzl (USA), Bismuth (France) and Ton That Tung (Vietnam). ${ }^{19-22}$ With better knowledge of the segmental anatomy, it was shown that parenchyma-sparing segmental resections were equally effective as classic lobar resections, and in this way more functional remnant liver was preserved. ${ }^{3,23,24}$ Also, anaesthetic care and liver transection techniques were modernized and improved over time. ${ }^{1,3,4,25,26}$ For instance, low central venous pressure during major hepatic resections reduced perioperative blood loss and had considerable impact on perioperative outcome. ${ }^{27}$

Over the last decades, it was shown in several large series that perioperative results became more encouraging, with operative mortality rates less than $5 \%$ in high volume centres. ${ }^{3,24,28}$ Due to these improvements in liver surgery which not only proved to prolong life but also to be a potentially curative treatment option for primary and metastatic cancers, ${ }^{29,30}$ liver surgery became standard of care for selected patients with primary and secondary hepatobiliary malignancies. Moreover, 
with the increasing improvements in the safety of hepatic resections, this evolved to the most effective treatment for some benign diseases. ${ }^{31}$

It is hard to pinpoint one discriminating factor that made the improvements in outcome possible. ${ }^{3}$ Many factors contribute to the gradually improved outcome. Most important factors in this regard are probably the better knowledge of hepatic anatomy and thus anatomically based resections, better patient selection, general improvements in operative and anaesthetic care and the development of hepatobiliary surgery as a distinct area of specialisation. ${ }^{3}$

\subsection{MALIGNANT LESIONS}

The liver has an important function as a detoxifying organ and due to the anatomical position in the abdomen; most gastrointestinal organs drain their venous blood to the liver. This makes the liver a frequent location of metastases from a variety of intra-abdominal and sometimes even extra-peritoneal primary cancers. Also, primary cancers can arise in the liver. Of these the hepatocellular carcinoma is the most common malignancy. With a normal functioning liver, resection is the treatment of choice for most of these malignant lesions.

Metastases of colorectal origin are the most frequent malignant lesions in the liver. With nearly one million new cases diagnosed each year and around half a million deaths annually, colorectal cancer is one of the most common causes of cancer related death worldwide. ${ }^{32}$ Over half of the patients with colorectal cancer will develop liver metastases. ${ }^{33}$ Moreover, up to $25 \%$ of these patients present with liver metastases at the same time of the primary diagnosis. ${ }^{34}$ Colorectal liver metastases may therefore be regarded as a major health problem. ${ }^{33}$

The only chance of long-term survival in patients with liver metastases is provided by the resection of these liver metastases, with 5 -year survival rates around $30-40 \% .{ }^{35}$ Until recently, however, few patients with malignant liver lesions were considered for partial hepatic resection. Due to the restricted resection criteria, only $10-20 \%$ of the patients with malignant lesions were selected. Palliative chemotherapy was offered for the remaining proportion of the patients, resulting in a median survival of 6-12months. ${ }^{8,36}$ Due to the increased safety of liver surgery, liver resection is currently also used for other metastases such as neuroendocrine tumours, ${ }^{37}$ sarcoma's, ${ }^{38}$ melanoma, ${ }^{39-41}$ gastric cancer ${ }^{42-44}$ and breast cancer. ${ }^{42,45,46}$

The selection criteria for liver resections were initially fairly strict: unilobar distribution, less than four metastases, maximum tumour size of $5 \mathrm{~cm}$ and tumour free margin of $1 \mathrm{~cm}$. These resection criteria have been evaluated over time and have gradually been abandoned, as these appeared to be not as important as previously assumed. ${ }^{47-49}$ Even in elderly patients and poor prognostic groups, complete tumour resection results in a good long-term survival. ${ }^{50-52}$ 
In the treatment of malignant liver disease, many improvements have been developed in recent years: new surgical strategies for safer resection (including two stage hepatectomy and portal vein embolisation), more effective chemotherapy, and additional techniques such as local ablation therapies to increase possible curative treatment. $^{53-58}$ The combination of these developments has led to an important progress and has resulted in more patients being considered suitable for liver resection to almost $30 \%{ }^{56}$ Better survival of patients with primary or metastatic liver cancer has been reported in recent years and liver resection is currently the only potentially curative treatment option.

\subsection{BENIGN LIVER LESIONS}

In case of malignant hepatic disease, surgical resection is currently felt justified despite a morbidity and mortality, which may be as high as $42 \%$ and $6.5 \%$ respectively. ${ }^{1,3,59-61}$ In case of benign hepatic disease, however, this decision remains more difficult. Due to the widespread use of imaging modalities such as ultrasonography, computed tomography (CT), and magnetic resonance imaging (MRI), benign hepatic masses are increasingly being identified. However, not all benign hepatic tumours require resection. Careful diagnosis with contrast enhanced CT or MRI needs to be performed first. Benign lesions can grossly be divided in solid and nonsolid lesions (table 2).

\section{History of hepatic surgery for benign lesions}

The first case of surgical resection for a presumably benign liver tumour was described in 1886 by Antonio Lius in Italy. ${ }^{62}$ Lius was the assistant of Theodore Escher who excised a pedunculated adenoma with the size of a child's head $(15.5 \mathrm{~cm}$ in greatest diameter) from the left liver lobe of 67-year-old women. An uncontrollable bleeding was encountered during the operation and the patient died several hours following surgery. The German surgeon Von Langenbuch was the first to perform a successful resection of a benign solid pedicled liver mass weighing 370 gram of the left liver in a 30-year-old woman who complained of abdominal discomfort in the years following her first child's birth in $1887 .{ }^{63}$ Post-operatively, secondary hemorrhage occurred due to a bleeding hilar vessel. This was managed at re-exploration and the patient survived. The course of symptoms and events in the latter case suggests the tumour was most likely a hepatocellular adenoma. 
Table 2. Most important benign liver lesions, divided in solid and non-solid lesions

\begin{tabular}{|c|c|c|}
\hline Solid lesions & Symptoms & Treatment \\
\hline Hepatocellular adenoma & $\begin{array}{l}\text { Variable: from incidental finding to severe } \\
\text { abdominal pain and shock in case of rupture }\end{array}$ & $\begin{array}{l}<5 \mathrm{~cm} \text { watchful waiting, } \\
\text { stop oral contraceptives } \\
>5 \mathrm{~cm} \text { resection to prevent } \\
\text { rupture and malignant } \\
\text { degeneration }\end{array}$ \\
\hline Focal Nodular Hyperplasia & Mostly incidental finding & Surgery rarely indicated \\
\hline Angiomyolipoma & Mostly incidental finding & Surgery rarely indicated \\
\hline $\begin{array}{l}\text { Nodular regenerative } \\
\text { hyperplasia }\end{array}$ & $\begin{array}{l}\text { Mostly asymptomatic, should be considered } \\
\text { in patients with clinical signs of portal hyper- } \\
\text { tension without evidence of cirrhosis }\end{array}$ & No proven treatment \\
\hline \multicolumn{3}{|l|}{ Non-solid lesions } \\
\hline Simple hepatic cyst & $\begin{array}{l}\text { Variable: from incidental finding to abdomi- } \\
\text { nal pain }\end{array}$ & $\begin{array}{l}\text { Surgery indicated only in } \\
\text { case of symptoms }\end{array}$ \\
\hline Biliary cystadenoma & $\begin{array}{l}\text { Variable: from incidental finding to abdomi- } \\
\text { nal pain }\end{array}$ & $\begin{array}{l}\text { Surgery may be indicated } \\
\text { (malignant degeneration) }\end{array}$ \\
\hline Biliary hamartoma & None & Surgery not indicated \\
\hline Cavernous haemangioma & Variable, depending on size & Surgery rarely indicated \\
\hline Hydatid disease & $\begin{array}{l}\text { Variable: from incidental finding to severe } \\
\text { abdominal pain and shock }\end{array}$ & $\begin{array}{l}\text { Surgery indicated to relieve } \\
\text { symptoms and to prevent } \\
\text { rupture }\end{array}$ \\
\hline
\end{tabular}

It is nowadays well established that small benign lesions compatible with a diagnosis of haemangioma, focal nodular hyperplasia (FNH) or hepatocelular adenomas (HCAs) are no indication for liver resection. Hepatocellular adenomas are considered the most important, albeit uncommon, benign tumours of the liver that mostly occur in women. They are known for their increased risk of haemorrhage and malignant transformation into hepatocellular carcinomas (HCC) if size exceeds 5 $\mathrm{cm}$. Therefore, surgical resection of HCAs is recommended for larger lesions. ${ }^{66}$ Focal nodular hyperplasia and haemangiomas have not been regarded as potentially premalignant lesions.

The first case report of malignant transformation of a HCA was published in 1981 by Tesluk and Lawrie. ${ }^{64}$ The patient was a 34 year old female with a large HCA measuring $16 \mathrm{~cm}$ in diameter. She first presented with tumour haemorrhage after which her oral contraceptive use was discontinued and the tumour subsequently shrank to a stable $5 \mathrm{~cm}$. Three years later a partial hepatectomy was performed when the tumour had reverted to its size at first presentation. Histological analysis revealed a well-differentiated HCC. The patient died of sepsis five weeks postoperatively. 
Foster and Berman were the first in 1994 to report an estimated risk of malignant transformation, as they found a frequency of $13 \%$ in their series of 13 patients. ${ }^{65}$ More recently, a systematic review of the literature of the past 40 years containing more than 1600 HCAs worldwide has been performed to investigate the actual rate of malignant degeneration of these benign tumours. ${ }^{66}$ Nowadays several other risk factors for malignant potential of HCAs apart from size have been identified. ${ }^{67-78}$ These are listed in table 3.

Table 3. Risk factors for malignant transformation of hepatocellular adenomas

Tumour size $>5 \mathrm{~cm}$

Presence of $\beta$-catenin activating mutation

Presence of liver cell dysplasia within HCA

Patients with glycogen storage disease

History of androgen or anabolic steroid intake

Male sex

Obesity/overweight

\section{Surgical treatment of hepatocellular adenomas}

The identification of several risk factors for malignant potential of HCAs in recent years provides better indications for surgical treatment of these presumably benign tumours. Also, the Bordeaux adenoma tumour markers (table 4) have greatly contributed to the subtype classification of HCAs and have given clearer insights into the pathological mechanism of malignant evolvement. ${ }^{73}$ More recently, MR imaging techniques have been shown to be of value in identifying premalignant HCAs. ${ }^{79,80}$ These advances in risk factor stratification together with tumour subtyping prior to hepatic surgery might aid in selecting HCAs at high risk of malignant evolvement for surgical resection. Unfortunately, routine performance of biopsy of an HCA has not been implemented yet owing to the risk of sampling error, bleeding, needle-track tumour seeding and the difficult interpretation of $\beta$-catenin staining. However, a change towards a more stringent selection process in the near future is inevitable and may imply a major reduction of the number of liver resections and thus morbidity and even mortality in a selected group of predominantly young patients. 
Table 4. Types of HCAs and their immunohistochemical markers

\begin{tabular}{llll}
\hline HCA type & Frequency (\%) & Malignant transformation & Markers \\
$\beta$-catenin activated & $10-15$ & Yes & $\beta$-catenin+/GS+ \\
HNF1 $\alpha$ inactivated & $30-50$ & Rarely & LFABP- \\
Inflammatory & 35 & No & SAA+/CRP+ \\
Unclassified & $5-10$ & No & None \\
\hline
\end{tabular}

CRP, C-reactive protein; GS, glutamine synthetase; HCA, hepatocellular adenoma; HNF1a, hepatocyte nuclear factor 1a; LFABP, liver-fatty acid binding protein; SAA, serum amyloid A; +, positive; -, negative. Table adapted with permission from Stoot et al. $2010 .^{66}$

\subsection{BENIGN CYSTIC LIVER LESIONS: HYDATID DISEASE}

Surgical treatment may also be indicated for infectious diseases of the liver such as benign lesions caused by the parasitic infection called Echinococcosis. Human echinococcosis is a zoonosis caused by larval forms (metacestodes) of Echinococcus (E.) tapeworms found in the small intestine of carnivores. Two species are of clinical importance - E. granulosus and E. multilocularis - causing cystic echinococcosis (CE) and alveolar echinococcosis (AE) in humans, respectively. ${ }^{81}$ Echinococcosis is endemic worldwide in large sheep-raising areas including Africa, the Mediterranean region of Europe, the Middle East, Asia, South America, Australia and New Zealand. $^{82-89}$ Human cystic echinococcosis is one of the most neglected parasitic diseases in the world. In many endemic regions most infected patients suffer considerably from this disease, usually because of the lack of treatment possibilities due to poor infrastructure and shortage of equipment and drugs. ${ }^{90,91}$ The incidence of hydatid disease in Western industrial nations is relatively low. ${ }^{86,87,92}$ Migration and travelling has led to an increase of the prevalence of this disease in Northern parts of Europe and North America. ${ }^{89,93}$ The diagnosis of hepatic echinococcosis can be made with a combination of patients' symptoms, liver imaging findings and the detection of Echinococcis-specific antibodies and microscopic or molecular examination of cyst fluid. The most frequent site for cystic lesions is the liver ( $60 \%$ of patients), followed by the lungs in about $20 \%$ of patients. ${ }^{87,94}$ The remaining lesions are found throughout the body. ${ }^{85,87,92,94,95}$

The natural course of this infection can be extremely variable. ${ }^{87,94}$ The hepatic cysts can spontaneously collapse, calcify or even disappear. These patients can remain symptom-free for years. It is not uncommon that the cysts are detected when abdominal imaging is performed for a different reason. On the other hand, the cysts can also steadily grow about $1-3 \mathrm{~cm}$ in diameter per year. ${ }^{89,92}$ They do not tend to grow infiltratively or destructively, but pressure or mass effects of the cysts can displace healthy tissue and organs. Thus, most patients present with symptoms from mechanical effects on other organs or structures, which can lead to pain in 
the upper right quadrant, hepatomegaly and jaundice, depending on the location and nature of the cysts. ${ }^{84,89,92,94}$ Infection of the cysts can result in sepsis and/or the formation of liver abscesses. A feared complication is rupture of hepatic hydatid cysts into the peritoneal cavity. This can result in serious anaphylaxis, sepsis and/or peritoneal dissemination. The content of the ruptured cyst can disseminate into the biliary tract leading to cholangitis or cholestasis, but also to the pleurae or lungs leading to pleural hydatidosis or bronchial fistula, respectively..$^{84,85,95}$

\section{History of hepatic surgery for hydatid disease}

Hydatid disease was already recognized by Hippocrates more than two millennia ago. This benign disease has been shown to act as a malignant disease as it has the tendency to disseminate to other organs and to cause a devastating disease sometimes even leading to death. The serious effects of this disease were known in the late 1880s, when Loretta performed the first left lateral liver resection for echinococosis in Bologna. ${ }^{8}$ In the recent years many developments have improved the course of hydatid disease, better medical therapy, improved surgical procedures and the development of minimally invasive techniques.

From a historical perspective, the main treatment option of hepatic hydatid disease was the open surgical approach with side packing and several radical or more conservative surgical techniques. ${ }^{89,92}$ This terminology in literature might be confusing. Conservative surgery means that tissue-sparing techniques are used; the hydatid cyst is evacuated and the pericyst is left in situ, while in radical procedures both the cyst and the pericyst are removed. The most common conservative techniques include simple tube drainage, marsupialization, capitonnage, deroofing, partial cystectomy or open or closed total cystectomy with or without omentoplasty. Conservative operations have good results regarding blood loss and length of hospital stay. ${ }^{96,97}$ In contrast, the cyst content and the entire pericystic membrane are removed in radical procedures; a total pericystectomy or liver resection (hemihepatectomy or lobectomy) is performed. ${ }^{83,87}$

Surgical interventions of hepatic hydatid cysts should completely remove the parasite. Also, prevention of intraoperative spilling of cyst content and saving healthy hepatic issue is of utmost importance. ${ }^{91,93,96}$ Spilling could not only lead to recurrence of hydatid disease, but before the introduction of the antihelmintic drugs, it could also lead to anaphylactic shock. Therefore, surgeons need to perform procedures with a focus on safe and complete exposure of the cyst, safe decompression of the cyst, safe evacuation of the cyst contents, sterilization of the cyst, treatment of biliary complications and management of the remaining cyst cavity. Especially in non-endemic areas where the number of operations is low, the technique needs to be safe and easily reproducible, with a low complication rate. In the former century, hydatid disease was operated with a high risk of morbidity and 
recurrence, possibly due to the spilling of cyst content during the operation. In the 1970s, Siadi developed a special cone, which was frozen to the cyst in order to reduce the risk of spilling cyst contents. This cone also simplified the disinfection of the cyst cavity (figure 3). ${ }^{98}$ Apart from the 'frozen-seal method', surgical treatment options may vary from conservative treatment (cystectomy) to radical treatment (complete open resection) to laparoscopic techniques. The debate on best surgical treatment is still ongoing: should this be conservative surgery or radical surgery in which the cyst is totally removed including the pericyst by total pericystectomy or partial hepatectomy or should it be the open or laparoscopic approach. ${ }^{96,97}$

Especially in non-endemic areas where the number of operations is low, it is of importance to perform a safe, reproducible technique with a low complication rate. In the Harbour Hospital in Rotterdam the 'frozen seal method' as surgical treatment for hydatid disease was used since 1981 (figure 3).

\section{Percutaneous treatments}

With the introduction of antihelmintic drugs, new possibilities for treatment arose. By using this medication, the risk of anaphylaxis became smaller and percutaneous treatments were developed. One of these treatments for hydatid disease is PAIR: Percutaneous Aspiration, Injection and Re-aspiration. In a recent meta-analysis of operative versus non-operative treatment (PAIR) of hepatic echinococcosis PAIR plus chemotherapy proved to be superior compared to surgery. The meta-analysis showed that PAIR was associated with improved efficacy, lower rates of morbidity, mortality, disease recurrence and also shorter hospital stay. ${ }^{86}$ 
(1)

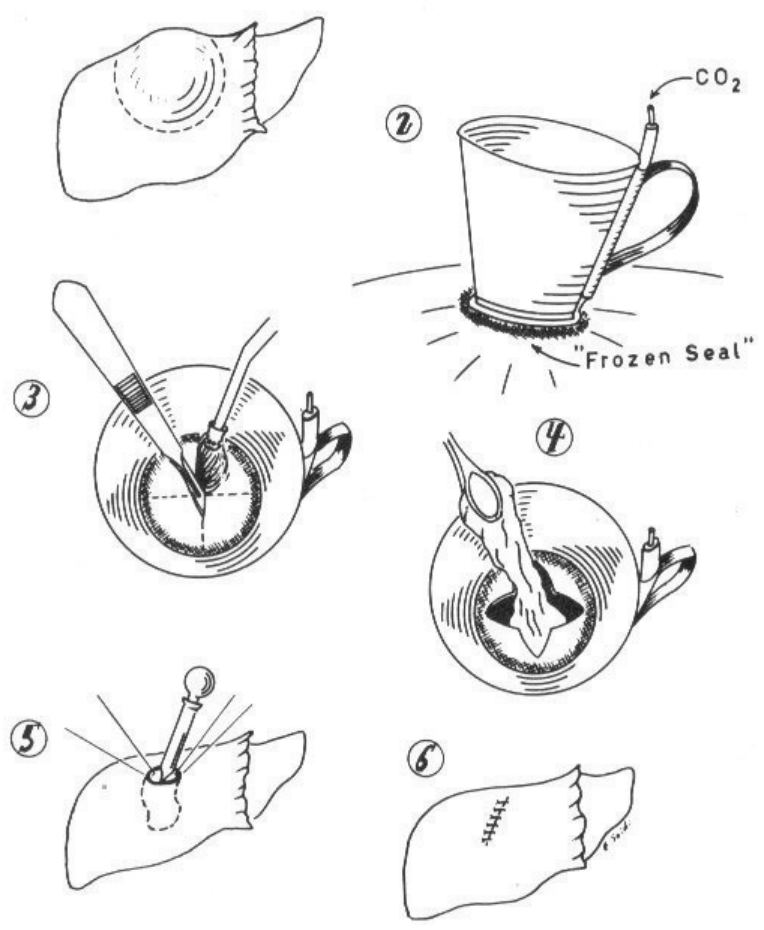

Figure 3, the 'frozen seal' technique for open surgery of hepatic hydatid disease. A metal cone is frozen on the surface of the liver producing a watertight frozen seal (2), the cyst wall is incised and the contents can be removed through the cone without the risk of uncontrolled rupture or spilling (3), the membrane together with all daughter cysts are excised (4) and the residual space is flushed for 2 minutes with a freshly prepared $0.5 \%$ silver nitrate solution to kill any remaining scolices (5), then the cavity is closed (6)

Reproduced with permission from Saidi ${ }^{98}$, Copyright Massachusetts Medical Society.

In conclusion, the main treatment options for hepatic cystic echinococcosis are threefold: medical therapy, surgery and percutaneous drainage (Puncture Aspiration Injection and Reaspiration, also known as PAIR) or a combination of these therapies. ${ }^{91,92}$ In the last revision of the WHO IWGE it was stated that surgery remains the cornerstone of treatment of hydatid disease, since it has the potential to remove the hydatid cyst and lead to complete cure. ${ }^{95}$ However, it is advised to evaluate surgical treatment carefully against other less invasive options such as percutaneous interventions. 


\subsection{MINIMALLY INVASIVE SURGERY}

To minimize the damage of treatment, laparoscopic surgery was introduced to avoid large incisions for many gastrointestinal operations in the previous century. After the first laparoscopic cholecystectomy, ${ }^{99,100}$ the number of indications for this minimally invasive approach increased. The outcome has encouraged surgeons to develop a laparoscopic technique for many procedures including liver resections. ${ }^{101}$ Although this type of surgery is technically more demanding and thereby timeconsuming $^{102,103}$ it proved to be beneficial for patients, with less pain and better recovery, compared to open liver surgery. ${ }^{104-106}$

\section{The history of laparoscopic surgery}

The fundamentals of laparoscopic surgery were laid down in the early twentieth century when the German surgeon Kelling reported in 1902 on the endoscopic visualization of the peritoneal cavity in an anesthetized dog using a Nitze cystoscope (1887). ${ }^{107}$ Following the introduction of endoscopic inspection of the abdominal contents in an animal model, fellow countryman Jacobeus started experimenting with laparoscopy in human cadavers as well as in living humans. In 1911 he reported on 80 laparoscopic examinations of the abdominal cavity. ${ }^{108,109}$ In the years thereafter the laparoscopic approach was enhanced with the introduction of illumination techniques, advancement in lens systems, the use of more than a single trocar and induction of pneumoperitoneum (Goetze and Veress). The era of therapeutic laparoscopy was then born, making it possible to minimize damage of treatment and avoid large incisions for many gastrointestinal operations. However, it was not until 1988 that the first experiences with laparoscopic cholecystectomy were published. ${ }^{106}$

At first, liver surgery was thought to be unsuitable for laparoscopic techniques since it might impose the risk of gas embolisms and major blood loss during transection of the liver. Also, sceptici pointed out the suspected risk of trocar site metastases in skin incisions. Gradually, as some expert centres progressively reported feasibility and safety, it became more popular.

This novel approach for liver resections was introduced during the Nineties of the past century. At first the procedure was only used for diagnostic laparoscopies and liver biopsies, later indications were extended to fenestration of liver cysts and anatomic liver resections. In 1992, Gagner et al. reported the first laparoscopic wedge resection of the liver. Only three years later, Cuesta and collegues were the first to perform two cases of limited laparoscopic liver surgery of segment II and IV in the Netherlands. ${ }^{110}$ The first laparoscopic left lateral bisegmentectomy of the liver was performed by the group of Azagra. ${ }^{111}$ Since then, several studies have 
reported the feasibility and safety of laparoscopic resections for liver tumours in centres with extensive experience in both hepatobiliary surgery and laparoscopic surgery. ${ }^{101,102,112-115}$

However, after its introduction, laparoscopic liver resection remained challenging because of the difficulties concerning safe mobilization and exposure of this fragile and heavy organ. Therefore, in the beginning only superficial and peripheral lesions in anterolateral segments were selected for the laparosopic approach. In recent times, centres with extensive experience in laparoscopy and hepatic surgery have also performed laparoscopic major hepatic resections with satisfactory outcomes. Importantly, no evidence of a compromised oncological clearance in laparoscopic liver resection has hitherto been found. ${ }^{105}$

\section{Advantages of laparoscopy}

The laparoscopic approach is said to have shifted the pain of the patient to the surgeon, as the latter had to obtain new operative skills and more demanding techniques. In fact laparoscopic surgery is a totally different concept of surgery. The conventional 3 dimensional field is inherently 2 dimensional, and the tactile feedback is impaired as compared to open surgery. Moreover, a full ambidexterity is required, as well as the skills to manipulate fragile structures with long instruments under minimal tactile feedback. Also, the surgeon becomes even more dependent on his team and instruments, as he will need experienced assistance for traction and camerawork and needs to trust the material even more compared to open surgery. For patients the most important presumed advantages of the laparoscopic procedure are reduced blood loss, ${ }^{104,105}$ less postoperative pain, ${ }^{103,112,116}$ earlier functional recovery, ${ }^{112,115}$ shorter postoperative hospital stay ${ }^{103,105,106,112,115-}$ 117 and improved cosmetic aspects. ${ }^{112,115}$ Reoperations are reported to be easier due to reduced adhesions. ${ }^{112,115-117}$ Also, open-close procedures with large incisions can be avoided when peritoneal metastases are detected at laparoscopy.

However, up till now no randomised controlled trials comparing the open and laparoscopic liver resection technique have been reported. This may well be one of the reasons why many surgeons remained reluctant to incorporate this new laparoscopic approach. The currently available evidence is primarily based on caseseries and identifies a technique that is reproducible with limited morbidity and mortality. In a consensus statement on laparoscopic liver resections, Buell $\mathrm{J}$ et $a l^{118}$ concluded that resection of segments 2 and 3 by the laparoscopic approach should be the standard of care.

However, implementation of new techniques mostly coincides with expanding costs, especially laparoscopic surgery, where expensive, disposable tools are required and more operation time is needed. ${ }^{119-121}$ Worldwide, both governmental organisations and health insurance companies have a major influence in cost regu- 
lation issues in health care. Hospital managements are increasingly strict concerning Operation Room (OR) cost. In daily practice, surgeons are required to make business plans and need to negotiate with management before introducing new techniques. In contrast to many feasibility studies on laparoscopic liver surgery, the data concerning the cost analysis of this surgical approach is limited. Still these techniques are being implemented worldwide without cost-analysis studies.

\subsection{ENHANCED RECOVERY AFTER SURGERY (ERAS) OR FAST-TRACK LIVER SURGERY}

Another recent development in elective liver surgery is the introduction of enhanced recovery programmes after surgery (ERAS), also referred to as fast track perioperative care. These multimodal enhanced recovery programmes proved to be beneficial in open colonic and liver surgery. ${ }^{122,123}$ The multimodal recovery programme is evidence based and combines several interventions in perioperative care to reduce the stress response and organ dysfunction with a focus on enhancing recovery. ${ }^{123,124}$ In patients undergoing colorectal surgery, the ERAS ${ }^{\circledR}$ programme enabled earlier recovery and consequently shorter length of hospital stay. ${ }^{123-126}$ Also, reduction of postoperative morbidity in patients undergoing intestinal resection was reported. ${ }^{127-130}$ In other fields of elective surgery similar programmes have also shown a reduction in hospital stay of several days. ${ }^{131,132}$

One of the pioneers of the fast track colonic surgery is the Danish surgeon Henrik Kehlet. He treated 60 consecutive patients with colonic resection in a fast track surgery programme and reported a median postoperative hospital stay of 2 days. At that time, patients undergoing a colonic resection usually required 5-10 days postoperative hospital stay. ${ }^{133,134}$ Previously, he stressed the importance of a multimodal approach in order to improve rehabilitation after surgery (figure 4). ${ }^{135}$ This rehabilitation programme after surgery combined a number of interventions to reduce stress of the surgical intervention, risk of organ dysfunction and loss of functional capacity. Stress induced organ dysfunction, pain, nausea and vomiting, ileus, hypoxemia and sleep disturbances, immobilisation and semistarvation had to be reduced. 


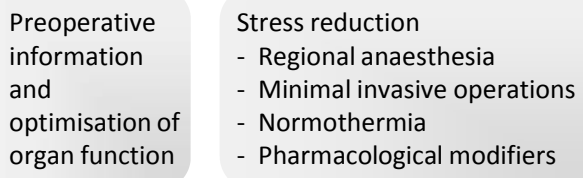

- Regional anaesth

- Minimal invasive operations

- Normothermia

- Pharmacological modifiers

Effective pain relief and prophylaxis of nausea and vomiting
Modification of perioperative care

- Early mobilisation

- Minimal use of tubes and drains

- Oral nutrition

Figure 4. Multimodal interventions may lead to a reduction in postoperative morbidity and improved recovery. ${ }^{135}$ Figure adapted with kind permission from Kehlet et al. 1997

Factors were identified that contribute to postoperative functional deterioration. These were actually traditional postoperative care principles such as use of drains, nasogastric tubes, fasting regimes and bed rest. Kehlet initiated a multimodal programme that abandoned the traditional care principles and introduced innovations such as carbohydrate loading before surgery, regional anaesthetic techniques, maintenance of normal temperature during surgery, minimally invasive or laparoscopic surgical techniques, optimal treatment of postoperative pain and prophylaxis of nausea and vomiting. ${ }^{125,136}$ This programme improved postoperative recovery, physical performance and pulmonary function and reduced hospital length of stay. $^{128}$

In collaboration with Kehlet, the Enhanced Recovery After Surgery (ERAS) group was initiated to investigate the perioperative care in four other hospitals (Royal Infirmary, Edinburgh, UK, The Karolinska Institutet at Ersta Hospital, Stockholm, Sweden, the University Hospital of Nothern Norway, Tromso, Noway and Maastricht University Medical Centre). ${ }^{137}$ Thus, with Kehlet's programme as a starting point, a new evidence based programme was developed incorporating different aspects leading to faster recovery. Preoperative counselling, perioperative intravenous fluid restriction, optimal pain relief preferably without the use of opioid analgesia, early oral nutrition, enforced mobilisation, no nasogastric tubes and no drains are the key elements of this protocol (figure 5). Since the colonic programme showed improvements in recovery, the liver surgeons of the ERAS ${ }^{\circledR}$ group (Maastricht, Edinburgh and Tromso) set up an ERAS-programme for every patient undergoing open liver resection ${ }^{122}$ (www.erassociety.org). 


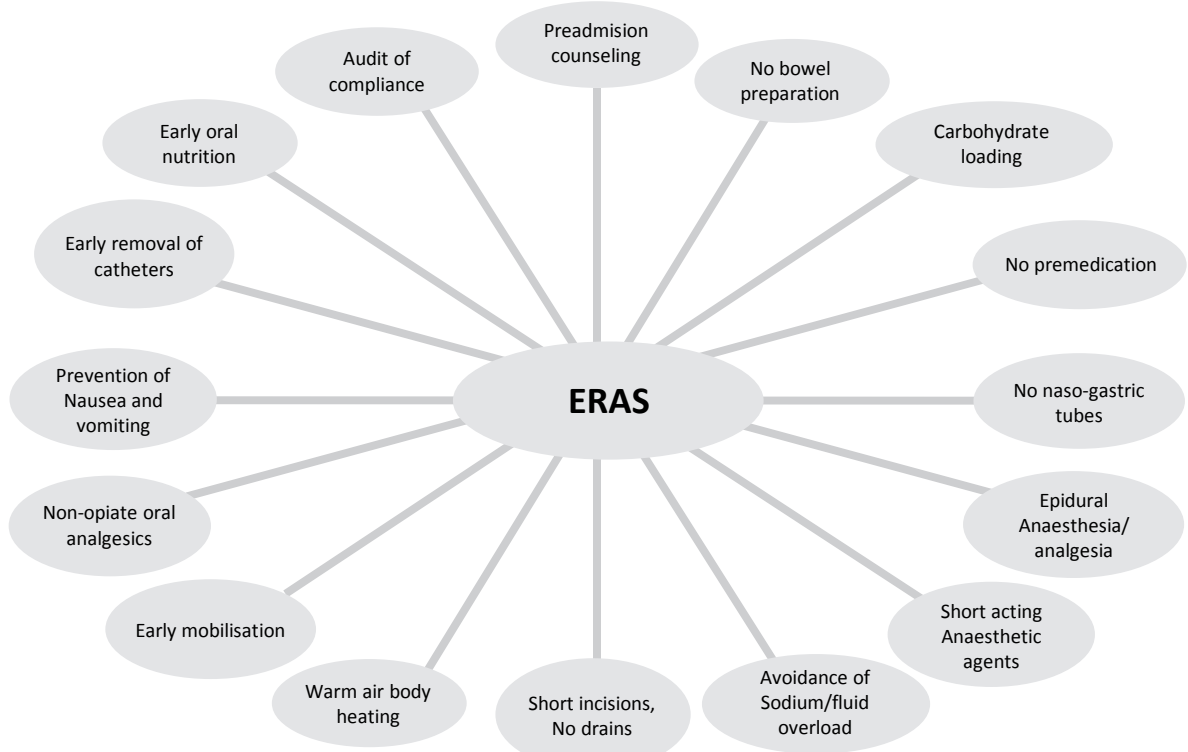

Figure 5. Important elements of the Enhanced Recovery After Surgery programme. ${ }^{124}$ Figure adapted with kind permission from Fearon et al. 2005

So far, the ERAS programmes have shown promising results with respect to improved recovery and outcome in open elective colorectal and liver surgery. ${ }^{122,123}$ One of the first studies on ERAS for liver surgery showed that the majority of patients treated within this multimodal enhanced recovery programme tolerated fluid within four hours of surgery and a normal diet on day 1 after surgery. As an effect of the accelerated functional recovery, these patients were discharged two days earlier than the patients treated with traditional care without significant differences in readmission, morbidity and mortality rates. ${ }^{122}$

These results were confirmed in a recent systematic review including seven studies on fast-track programmes for hepatopancreatic resections, incorporating more than 550 patients treated in fast track setting. ${ }^{138}$ This study showed that the primary hospital stay was reduced significantly after the introduction of a multimodal perioperative care programme for open liver surgery. ${ }^{138}$ Moreover, there were no significant differences in rates of readmission, morbidity and mortality.

For solid tumours in the liver, the open approach for resection is gradually replaced by the laparoscopic technique in many expert centres worldwide. However, the added value of a fast track ERAS programme in laparoscopic liver surgery specifically has hitherto not been elucidated. 


\subsection{FUTURE PERSPECTIVES}

As for the recent developments in the treatment of liver diseases, these can be mainly divided into surgical and non-surgical treatment modalities. Developments in surgical treatment can be divided in true surgical and perioperative care improvements. The focus will be on the surgical treatments in this chapter, but some thoughts will also be spent on the non-surgical treatment modalities, an interesting and expanding field of expertise.

For malignant liver tumours, the majority of which are colorectal liver metastases, the main concern is the resectability when colorectal cancer is diagnosed. Colorectal cancer is one of the most common causes of cancer related death worldwide $^{32}$ and more than half of patients with colorectal cancer will develop liver metastases. $^{33}$ Unfortunately, only $20 \%$ of the patients can be treated with surgical resection of these liver metastases. ${ }^{139}$ The remaining $80 \%$ of the patients present with lesions, which are not suitable for a safe resection. This can be caused by large diameters of the lesions, location of the lesion near vascular and biliary structures and extrahepatic disease. Also, the number of lesions can be the cause of nonresectability: resection can only be carried out safely if $25-30 \%$ of functioning liver remains after resection. ${ }^{140}$ The non-surgical treatment by means of chemotherapy for the patients with unresected liver metastases has proven very successful in decreasing the size and number of liver lesions. It was shown that new chemotherapy regimens could change the previously unresectable liver metastases into resectable liver disease. ${ }^{141}$ With neo-adjuvant chemotherapy more patients with colorectal liver metastases can be offered a treatment with curative intent. ${ }^{141}$ It was concluded that neoadjuvant chemotherapy enables liver resection in some patients with initially unresectable colorectal metastases. Long-term survival proved to be similar to that reported for a priori surgical candidates. ${ }^{50}$ As for the future perspective of chemotherapy, neoadjuvant treatment will improve curability and long-term survival for selected patients.

Other non-surgical therapies for malignant liver disease are external irradiation (whole liver irradiation), ${ }^{142,143}$ stereotactic liver irradiation ${ }^{144-147}$ and injectable small radioactive particles that irradiate the tumours within the liver (e.g. Yttrium-90 $\left({ }^{90} \mathrm{Y}\right.$ ) radio-embolisation, ${ }^{148,149}$ radioactive holmium microspheres ${ }^{150,151}$ ). These modalities may have curative potential but future studies have to be awaited. Another attractive field of development are the thermal ablative therapies for unresectable liver metastases. These ablative thermal therapies can be used either percutaneously or in adjunct with surgery and have shown to decrease focal liver lesions. ${ }^{152-}$ 155 Microwave ablation is a tumour destruction method to treat patients with unresectable liver lesions. ${ }^{154}$ It can be used with a single insertion of the probe and it was shown to be a safe and effective method for treating unresectable hepatic 
tumours, with a low rate of local recurrence. ${ }^{155}$ Overall survival is comparable to alternative ablation modalities. ${ }^{154}$

As for surgical treatments, different treatment strategies have been developed to increase the number of patients suitable for surgery as described earlier. Current research has focussed on improving resectability in terms of the quantity of resected liver tissue, but at the same time studies focussed on reducing perioperative distress in patients undergoing liver resections by multimodal perioperative treatment protocols and minimally invasive surgery. Since the introduction of laparoscopic liver surgery in 1992, more liver resections have been performed with this minimally invasive approach for primary and secondary malignant liver lesions. ${ }^{114,156,157}$ For future perspectives, some gain might be expected from even less invasive modalities as the first reports on single incision laparoscopic resections have been presented. ${ }^{158-160}$ Also, a two-stage laparoscopic approach for malignant liver disease and the robotic approach for liver resections have been published. ${ }^{161-}$ 163

\section{Improvements in preoperative planning}

An important way to improve the outcome in liver surgery is to prevent liver resection related complications. One of the main feared complications in liver surgery remains postresectional liver failure. This major complication may occur if the extent of tumour involvement requires major liver resection (3 or more segments), leaving a small postoperative remnant liver. ${ }^{3,164,165}$ Due to impaired liver function this may even result in mortality. Obviously, limiting the liver resection, in order to leave enough liver remnant volume for proper function of the liver, can prevent this. However, major hepatectomies are performed increasingly more often, mainly because indications for liver resection are continuously being extended. Former contraindications such as bilobar disease, number of metastases and even extrahepatic disease have been abandoned gradually and compromised liver function may be expected after aggressive induction chemotherapy. Consequently, postoperative remnant liver volume and function have become the main determinants of resectability. ${ }^{166-168}$ In order to improve outcome in extended resections and thus to prevent postoperative liver failure after liver resection, a reliable volumetric assessment of the part of the liver to be resected as well as future residual liver volume should be a critical part of preoperative evaluation particularly. The safety of liver resection may increase if an estimate of minimal remnant liver volume is obtained via CT-volumetry. ${ }^{164,169}$

The utility of existing professional image-processing software is often limited by costs, lack of flexibility and specific hardware requirements such as coupling to a CT-scanner. In addition, the intended operation should be known to the investiga- 
tor to predict the remnant liver volume accurately and requires the expertise of a liver surgeon. Therefore, CT-volumetry has hitherto been a multidisciplinary modality requiring the efforts of dedicated surgeons and radiologists and expensive software. Prospective CT-volumetric analysis of the liver on a Personal Computer performed by the operating surgeon in patients undergoing major liver would greatly enhance this preoperative assessment. ImageJ is a free, open-source Java-based image processing software programme developed by the National Institute of Health (NIH) and may be used for this purpose. ${ }^{170}$ Osirix ${ }^{\circledR}$ is Apple's version for image analysis and has been tested for CT volumetry of the liver. ${ }^{171}$ It is also a freely available, user-friendly software system, which can be used for virtual liver resections and volumetric analysis. ${ }^{171}$

As more major liver resections are performed, it is becoming more important to perform liver volumetry.

\section{Future studies}

As discussed previously in this chapter, the recent developments in liver surgery include the introduction of laparoscopic surgery and enhanced recovery programmes, which focus on improvement of postoperative recovery and/or shorter hospital length of stay. A significantly accelerated recovery after open liver resection was previously reported when patients were managed within a multimodal ERAS protocol. Median hospital length of stay was reduced from 8 to 6 days $(25 \%) .{ }^{122}$ Moreover, since there was a delay between recovery and discharge of the patients a further reduction of stay should be possible. Regarding the results of previous, non-randomised studies and case series, it seems that laparoscopic left lateral liver sectionectomy is associated with shorter hospital length of stay, less post-operative pain, better quality of life and a faster recovery. ${ }^{172}$ In most trials aiming at a reduction of hospital length of stay, surgery and/or perioperative management are not standardised. No randomised trials have hitherto been reported to study the added value of ERAS and/or laparoscopy for liver surgery. There is a need for a randomised controlled trial covering these aspects of improving the recovery and outcome of liver surgery.

Especially for benign lesions the hepatic resectional therapy has to be questioned or at least alternative therapies have to be explored as the surgical treatment still carries a certain risk of morbidity and even mortality. Hepatocellular adenomas (HCAs) are rare benign tumours mostly occurring mainly in young women. For $H_{C A}$ 's larger than $5 \mathrm{~cm}$, resection is suggested mainly because of the risk of rupture and malignant transformation. Previous results showed that selective arterial embolisation of haemorrhaging and non-haemorrhaging HCAs was effective as acute treatment to stop life-threatening hemorrhage but might also have its effect on the 
size of the HCAs. This minimally invasive method for acute treatment and its possible effect of downsizing hepatic adenomas warrant future studies.

\subsection{OUTLINE OF THIS THESIS}

The scope of the current thesis was to investigate ways to reduce patient harm in the surgical treatment for benign liver disease. 'Primum non nocere' for liver surgery by means of reducing surgical trauma by using:

- novel techniques for life threatening haemorraging of liver lesions,

- minimally invasive liver surgery,

- improved perioperative care protocols.

\section{Part 2}

A challenge that may be encountered during differential diagnosis of a focal liver lesion is the distinction between metastatic disease and other focal lesions (Chapter 2). As described previously, echinococcosis or hydatid disease of the liver remains endemic in the world and is an imported disease in the Netherlands. In chapter 3 , treatment and outcome of patients treated surgically for hydatid disease in a single centre in the Netherlands is evaluated. Since a decrease in number of operations was noticed for this rare disease of the liver, a study was initiated to investigate the true incidence of hepatic echinococcosis in a non-endemic area as the Netherlands. Since this disease carries no legal obligation to report, there are no reliable incidence and prevalence numbers available. Therefore, collaboration was established with the Dutch organisation that maintains the pathologic database (PALGA) in the Netherlands and also with the several institutes with distinct serology databases (chapter 4). To investigate treatment options for this rare disease, a survey was conducted to explore the opinions of experts concerning treatment of echinococcosis (chapter 4).

\section{Part 3}

This part is built up of 4 chapters and deals with the management of hepatocellular adenomas. After the introduction, the presentation of a case with life threatening haemorrhage is described from diagnosis to treatment. This report is remarkable as it is the first case reported in literature of a liver rupture where selective arterial embolisation is performed successfully in a young female patient just after delivery (chapter 5). This publication notes the transition in selective operative treatment of liver rupture towards scarless treatment and marks the beginning of this thesis. ${ }^{173}$ Chapter 6 deals with the debate whether routine resection of hepatocellular ade- 
nomas after haemorrhage and consequent selective arterial embolisation is useful. ${ }^{174}$ Having shown no viable adenoma tissue in resected specimen after resection, the rationale for follow-up instead of resection of ruptured liver lesions is discussed.

In chapter 7 selective arterial embolisation as emergency treatment for ruptured hepatocellular adenomas in patients with life threatening haemorrhage is evaluated. ${ }^{175}$ In Leiden University Medical Centre (LUMC), this was used as an initial therapy to stabilise hemodynamically unstable patients with haemorrhaging ruptured hepatocellular adenomas. Success and complications of this minimally invasive tool are discussed and long-term effect on the size of the adenomas is displayed. This less invasive approach might reduce morbidity and mortality, as the acute resection accounts for mortality up to $10 \% .{ }^{176,177}$ The effect of selective arterial embolisation on the size and growth of these adenomas is also investigated.

The risk of malignant transformation of hepatocellular adenomas (HCA) into hepatocellular carcinomas ( $\mathrm{HCC}$ ) is discussed in chapter 8 . This has been reported repeatedly and is considered one of the main reasons for resection. However, its actual risk is currently unknown. The literature was systematically reviewed the literature to estimate the frequency of malignant transformation of HCA's and to discuss its clinical implications. ${ }^{66}$

\section{Part 4}

Different aspects of the minimally invasive or laparoscopic approach for liver resections are evaluated in part 4. Before implementing any new technique, most important aspects such as feasibility, safety and cost-effectiveness should be evaluated. In the era of expanding costs of healthcare, a single centre study was conducted to assess the feasibility, safety and cost analysis of introducing a laparoscopic liver surgery programme for left sided liver lesions (chapter 9). Notably, a study where both minimally invasive surgery and a fast track programme were investigated has hitherto not been reported. Therefore, in a multicentre pilot study, the added value of an Enhanced Recovery After Surgery (ERAS) programme for laparoscopic liver resections for solid tumours was evaluated (chapter 10 ).

In chapter 11 the initial experience with laparoscopic liver resections in the Netherlands is reported. More specifically, the laparoscopic approach was compared in a case-control study with the open approach of a fairly standardised hepatectomy, in order to provide the second best level of evidence. Moreover, the aim was to identify independent factors determining length of hospital stay in laparoscopic liver surgery. Feasibility, safety and outcome of these procedures were also assessed. Lastly, the need for a randomised trial for the worldwide adoption of laparoscopic liver surgery was investigated by means of an international survey among the hepatic surgical community (chapter 12). 


\section{Part 5}

In this chapter the preoperative planning for major liver resections with the software packages, ImageJ and Osirix ${ }^{\circledR}$ in chapter 13 was evaluated. These have not yet been validated for CT-volumetry of the liver resection specimen prospectively prior to surgery. Therefore, the objective of this study was to compare ImageJ and OsiriX $^{\circledR}$ in performing prospective CT-volumetric analysis of the liver on a Personal Computer in patients undergoing major liver resection. In addition, the present research also aimed to compare the prospective measurements of ImageJ and Osirix ${ }^{\circledR}$ to $\mathrm{CT}$-volumetry performed by a radiologist using classical radiological software (iNtuition ${ }^{\circledR}$ ) linked to a CT-scanner system.

In chapter 14 an outline for a randomised trial is proposed in order to investigate the proposed algorithm of the systematic review (chapter 8) in a more structured manner.

Since there is no evidence of the added value of ERAS and laparoscopic liver surgery in a randomised controlled setting, a study was initiated to investigate the added value of laparoscopy compared to open liver surgery in left lateral liver sectionectomy within an ERAS programme in terms of functional recovery, hospital length of stay and postoperative morbidity (chapter 15).

\section{REFERENCES}

1. Poon RT, Fan ST, Lo CM, et al. Improving perioperative outcome expands the role of hepatectomy in management of benign and malignant hepatobiliary diseases: analysis of 1222 consecutive patients from a prospective database. Ann Surg 2004; 240: 698-708; discussion 708-610.

2. Cescon M, Vetrone G, Grazi GL, et al. Trends in perioperative outcome after hepatic resection: analysis of 1500 consecutive unselected cases over 20 years. Ann Surg 2009; 249: 995-1002.

3. Jarnagin WR, Gonen M, Fong Y, et al. Improvement in perioperative outcome after hepatic resection: analysis of 1,803 consecutive cases over the past decade. Ann Surg 2002; 236: 397-406; discussion 406-397.

4. Tsao JI, Loftus JP, Nagorney DM, et al. Trends in morbidity and mortality of hepatic resection for malignancy. A matched comparative analysis. Ann Surg 1994; 220: 199-205.

5. Foster JH. History of liver surgery. Arch Surg 1991; 126: 381-387.

6. Glisson F. Anatomia Hepatis. 1654; London, England.

7. Cantlie J. On a new arrangement of the right and left lobes of the liver. J. Anat. Physiol. 1898; 32: 49.

8. Hardy KJ. Liver surgery: the past 2000 years. Aust N Z J Surg 1990; 60: 811-817.

9. Pringle JH. V. Notes on the Arrest of Hepatic Hemorrhage Due to Trauma. Ann Surg 1908; 48: 541549.

10. Couinaud C. Le Foie. Etudes anatomiques et chirurgicales. 1957. Paris: Masson.

11. Guyton A, Hall J. The liver as an organ. Textbook of Medical Physiology, Philadelphia; WB Saunders 1996; 883-888.

12. Taub R. Liver regeneration: from myth to mechanism. Nat Rev Mol Cell Biol 2004; 5: 836-847.

13. van de Poll MC, Wigmore SJ, Redhead DN, et al. Effect of major liver resection on hepatic ureagenesis in humans. Am J Physiol Gastrointest Liver Physiol 2007; 293: G956-962. 
14. Lortat-Jacob JL, Robert HG, Henry C. [Excision of the right lobe of the liver for a malignant secondary tumor]. Arch Mal Appar Dig Mal Nutr 1952; 41: 662-667.

15. Foster JH, Berman MM. Solid liver tumors. Major Probl Clin Surg 1977; 22: 1-342.

16. Ekberg $\mathrm{H}$, Tranberg KG, Andersson $\mathrm{R}$, et al. Determinants of survival in liver resection for colorectal secondaries. Br J Surg 1986; 73: 727-731.

17. Adson MA, van Heerden JA, Adson $\mathrm{MH}$, et al. Resection of hepatic metastases from colorectal cancer. Arch Surg 1984; 119: 647-651.

18. Simmonds PC, Primrose JN, Colquitt JL, et al. Surgical resection of hepatic metastases from colorectal cancer: a systematic review of published studies. Br J Cancer 2006; 94: 982-999.

19. Iwatsuki S, Shaw BW, Jr., Starzl TE. Experience with 150 liver resections. Ann Surg 1983; 197: 247253.

20. Bismuth $\mathrm{H}$, Houssin D, Castaing D. Major and minor segmentectomies "reglees" in liver surgery. World J Surg 1982; 6: 10-24.

21. Bismuth H. Surgical anatomy and anatomical surgery of the liver. World J Surg 1982; 6: 3-9.

22. Tung TT. Les resections majeures et mineures du foie. 1979. Paris: Masson.

23. Billingsley KG, Jarnagin WR, Fong $Y$, et al. Segment-oriented hepatic resection in the management of malignant neoplasms of the liver. J Am Coll Surg 1998; 187: 471-481.

24. Fan ST, Lo CM, Liu CL, et al. Hepatectomy for hepatocellular carcinoma: toward zero hospital deaths. Ann Surg 1999; 229: 322-330.

25. Farid H, O'Connell T. Hepatic resections: changing mortality and morbidity. Am Surg 1994; 60: 748752.

26. Poon RT. Recent advances in techniques of liver resection. Surg Technol Int 2004; 13: 71-77.

27. Melendez JA, Arslan V, Fischer ME, et al. Perioperative outcomes of major hepatic resections under low central venous pressure anesthesia: blood loss, blood transfusion, and the risk of postoperative renal dysfunction. J Am Coll Surg 1998; 187: 620-625.

28. Fong $\mathrm{Y}$, Fortner J, Sun RL, et al. Clinical score for predicting recurrence after hepatic resection for metastatic colorectal cancer: analysis of 1001 consecutive cases. Ann Surg 1999; 230: 309-318; discussion 318-321.

29. Adam R, Wicherts DA, de Haas RJ, et al. Patients with initially unresectable colorectal liver metastases: is there a possibility of cure? J Clin Oncol 2009; 27: 1829-1835.

30. Tomlinson JS, Jarnagin WR, DeMatteo RP, et al. Actual 10-year survival after resection of colorectal liver metastases defines cure. J Clin Oncol 2007; 25: 4575-4580.

31. Charny CK, Jarnagin WR, Schwartz LH, et al. Management of 155 patients with benign liver tumours. Br J Surg 2001; 88: 808-813.

32. Boyle P, Leon ME. Epidemiology of colorectal cancer. Br Med Bull 2002; 64: 1-25.

33. Steele G, Jr., Ravikumar TS. Resection of hepatic metastases from colorectal cancer. Biologic perspective. Ann Surg 1989; 210: 127-138.

34. Manfredi S, Lepage C, Hatem C, et al. Epidemiology and management of liver metastases from colorectal cancer. Ann Surg 2006; 244: 254-259.

35. Scheele J, Stang R, Altendorf-Hofmann A, et al. Resection of colorectal liver metastases. World J Surg 1995; 19: 59-71.

36. Thirion $\mathrm{P}$, Michiels S, Pignon JP, et al. Modulation of fluorouracil by leucovorin in patients with advanced colorectal cancer: an updated meta-analysis. J Clin Oncol 2004; 22: 3766-3775.

37. Mayo SC, de Jong MC, Pulitano C, et al. Surgical management of hepatic neuroendocrine tumor metastases: results from an international multi-institutional analysis. Ann Surg Oncol 2010; 17: 3129-3136.

38. Rehders $\mathrm{A}$, Peiper $\mathrm{M}$, Stoecklein $\mathrm{NH}$, et al. Hepatic metastasectomy for soft-tissue sarcomas: is it justified? World J Surg 2009; 33: 111-117.

39. Mondragon-Sanchez R, Barrera-Franco JL, Cordoba-Gutierrez $\mathrm{H}$, et al. Repeat hepatic resection for recurrent metastatic melanoma. Hepatogastroenterology 1999; 46: 459-461. 


\section{Chapter 1}

40. Pawlik TM, Zorzi D, Abdalla EK, et al. Hepatic resection for metastatic melanoma: distinct patterns of recurrence and prognosis for ocular versus cutaneous disease. Ann Surg Oncol 2006; 13: 712720.

41. Frenkel S, Nir I, Hendler K, et al. Long-term survival of uveal melanoma patients after surgery for liver metastases. Br J Ophthalmol 2009; 93: 1042-1046.

42. Karavias DD, Tepetes K, Karatzas $\mathrm{T}$, et al. Liver resection for metastatic non-colorectal nonneuroendocrine hepatic neoplasms. Eur J Surg Oncol 2002; 28: 135-139.

43. Hirai I, Kimura W, Fuse A, et al. Surgical management for metastatic liver tumors. Hepatogastroenterology 2006; 53: 757-763.

44. Makino $\mathrm{H}$, Kunisaki $\mathrm{C}$, Izumisawa $\mathrm{Y}$, et al. Indication for hepatic resection in the treatment of liver metastases from gastric cancer. Anticancer Res 2010; 30: 2367-2376.

45. Lermite $E$, Marzano $E$, Chereau $E$, et al. Surgical resection of liver metastases from breast cancer. Surg Oncol 2009; 19: 79-84.

46. Sakamoto $\mathrm{Y}$, Yamamoto J, Yoshimoto $\mathrm{M}$, et al. Hepatic resection for metastatic breast cancer: prognostic analysis of 34 patients. World J Surg 2005; 29: 524-527.

47. Figueras J, Burdio F, Ramos E, et al. Effect of subcentimeter nonpositive resection margin on hepatic recurrence in patients undergoing hepatectomy for colorectal liver metastases. Evidences from 663 liver resections. Ann Oncol 2007; 18: 1190-1195.

48. Figueras J, Torras J, Valls C, et al. Surgical resection of colorectal liver metastases in patients with expanded indications: a single-centre experience with 501 patients. Dis Colon Rectum 2007; 50: 478-488.

49. Khatri VP, Petrelli NJ, Belghiti J. Extending the frontiers of surgical therapy for hepatic colorectal metastases: is there a limit? J Clin Oncol 2005; 23: 8490-8499.

50. Adam R, Avisar E, Ariche A, et al. Five-year survival following hepatic resection after neoadjuvant therapy for nonresectable colorectal. Ann Surg Oncol 2001; 8: 347-353.

51. Figueras J, Ramos E, Lopez-Ben S, et al. Surgical treatment of liver metastases from colorectal carcinoma in elderly patients. When is it worthwhile? Clin Transl Oncol 2007; 9: 392-400.

52. Adam R, Frilling A, Elias $\mathrm{D}$, et al. Liver resection of colorectal metastases in elderly patients. $\mathrm{Br} \mathrm{J}$ Surg 2010; 97: 366-376.

53. de Haas RJ, Wicherts DA, Adam R. Resection of colorectal liver metastases with extrahepatic disease. Dig Surg 2008; 25: 461-466.

54. Adam R, de Haas RJ, Wicherts DA, et al. Is hepatic resection justified after chemotherapy in patients with colorectal liver metastases and lymph node involvement? J Clin Oncol 2008; 26: 36723680.

55. Wicherts DA, de Haas RJ, Andreani P, et al. Impact of portal vein embolization on long-term survival of patients with primarily unresectable colorectal liver metastases. Br J Surg 2010; 97: 240-250.

56. Choti MA, Sitzmann JV, Tiburi MF, et al. Trends in long-term survival following liver resection for hepatic colorectal metastases. Ann Surg 2002; 235: 759-766.

57. de Haas RJ, Wicherts DA, Flores $\mathrm{E}$, et al. R1 resection by necessity for colorectal liver metastases: is it still a contraindication to surgery? Ann Surg 2008; 248: 626-637.

58. Wicherts DA, Miller R, de Haas RJ, et al. Long-term results of two-stage hepatectomy for irresectable colorectal cancer liver metastases. Ann Surg 2008; 248: 994-1005.

59. Virani S, Michaelson JS, Hutter MM, et al. Morbidity and mortality after liver resection: results of the patient safety in surgery study. J Am Coll Surg 2007; 204: 1284-1292.

60. Dixon E, Schneeweiss S, Pasieka JL, et al. Mortality following liver resection in US medicare patients: does the presence of a liver transplant programme affect outcome? J Surg Oncol 2007; 95: 194-200.

61. Fong Y, Blumgart LH, Cohen AM. Surgical treatment of colorectal metastases to the liver. CA Cancer J Clin 1995; 45: 50-62.

62. Lius A. Di un adenoma del fegato. Gazz delle cliniche 1886; 23. 
63. Langenbuch C. Ein Fall von Resektion eines linksseitigen Schnurlappens der Leber. Berl Klin Woschenschr 1888; 25: 37-38.

64. Tesluk H, Lawrie J. Hepatocellular adenoma. Its transformation to carcinoma in a user of oral contraceptives. Arch Pathol Lab Med 1981; 105: 296-299.

65. Foster JH, Berman MM. The malignant transformation of liver cell adenomas. Arch Surg 1994; 129 : 712-717.

66. Stoot JH, Coelen RJ, De Jong MC, et al. Malignant transformation of hepatocellular adenomas into hepatocellular carcinomas: a systematic review including more than 1600 adenoma cases. HPB (Oxford) 2010; 12: 509-522.

67. Bioulac-Sage P, Taouji S, Possenti L, et al. Hepatocellular adenoma subtypes: the impact of overweight and obesity. Liver Int; 2012.

68. Dokmak S, Paradis V, Vilgrain V, et al. A Single Centre Surgical Experience of 122 Patients with Single and Multiple Hepatocellular Adenomas. Gastroenterology 2009.

69. Franco LM, Krishnamurthy V, Bali D, et al. Hepatocellular carcinoma in glycogen storage disease type la: a case series. J Inherit Metab Dis 2005; 28: 153-162.

70. Gorayski $\mathrm{P}$, Thompson $\mathrm{CH}$, Subhash HS, et al. Hepatocellular carcinoma associated with recreational anabolic steroid use. Br J Sports Med 2008; 42: 74-75; discussion 75.

71. Labrune $P$, Trioche $P$, Duvaltier I, et al. Hepatocellular adenomas in glycogen storage disease type I and III: a series of 43 patients and review of the literature. J Pediatr Gastroenterol Nutr 1997; 24: 276-279.

72. Velazquez I, Alter BP. Androgens and liver tumors: Fanconi's anemia and non-Fanconi's conditions. Am J Hematol 2004; 77: 257-267.

73. Zucman-Rossi J, Jeannot E, Nhieu JT, et al. Genotype-phenotype correlation in hepatocellular adenoma: new classification and relationship with HCC. Hepatology 2006; 43: 515-524.

74. Anthony PP, Vogel CL, Barker LF. Liver cell dysplasia: a premalignant condition. J Clin Pathol 1973; 26: 217-223.

75. Ho JC, Wu PC, Mak TK. Liver cell dysplasia in association with hepatocellular carcinoma, cirrhosis and hepatitis B surface antigen in Hong Kong. Int J Cancer 1981; 28: 571-574.

76. Lee RG, Tsamandas AC, Demetris AJ. Large cell change (liver cell dysplasia) and hepatocellular carcinoma in cirrhosis: matched case-control study, pathological analysis, and pathogenetic hypothesis. Hepatology 1997; 26: 1415-1422.

77. Su Q, Benner A, Hofmann WJ, et al. Human hepatic preneoplasia: phenotypes and proliferation kinetics of foci and nodules of altered hepatocytes and their relationship to liver cell dysplasia. Virchows Arch 1997; 431: 391-406.

78. Tao LC. Oral contraceptive-associated liver cell adenoma and hepatocellular carcinoma. Cytomorphology and mechanism of malignant transformation. Cancer 1991; 68: 341-347.

79. van Aalten SM, Thomeer MG, Terkivatan $\mathrm{T}$, et al. Hepatocellular adenomas: correlation of MR imaging findings with pathologic subtype classification. Radiology 2011; 261: 172-181.

80. Laumonier H, Bioulac-Sage $\mathrm{P}$, Laurent $\mathrm{C}$, et al. Hepatocellular adenomas: magnetic resonance imaging features as a function of molecular pathological classification. Hepatology 2008; 48: 808818.

81. Brunetti E, Kern P, Vuitton DA. Expert consensus for the diagnosis and treatment of cystic and alveolar echinococcosis in humans. Acta Trop 2010; 114: 1-16.

82. Ammann RW, Eckert J. Cestodes. Echinococcus. Gastroenterol Clin North Am 1996; 25: 655-689.

83. Dziri C, Haouet K, Fingerhut A. Treatment of hydatid cyst of the liver: where is the evidence? World J Surg 2004; 28: 731-736.

84. Gourgiotis $S$, Stratopoulos $C$, Moustafellos $P$, et al. Surgical techniques and treatment for hepatic hydatid cysts. Surg Today 2007; 37: 389-395.

85. Khuroo MS, Wani NA, Javid G, et al. Percutaneous drainage compared with surgery for hepatic hydatid cysts. N Engl J Med 1997; 337: 881-887. 


\section{Chapter 1}

86. Smego RA, Jr., Bhatti S, Khaliq AA, et al. Percutaneous aspiration-injection-reaspiration drainage plus albendazole or mebendazole for hepatic cystic echinococcosis: a meta-analysis. Clin Infect Dis 2003; 37: 1073-1083.

87. Smego RA, Jr., Sebanego P. Treatment options for hepatic cystic echinococcosis. Int J Infect Dis 2005; 9: 69-76.

88. Yagci G, Ustunsoz B, Kaymakcioglu N, et al. Results of surgical, laparoscopic, and percutaneous treatment for hydatid disease of the liver: 10 years experience with 355 patients. World J Surg 2005; 29: 1670-1679.

89. Sayek I, Tirnaksiz MB, Dogan R. Cystic hydatid disease: current trends in diagnosis and management. Surg Today 2004; 34: 987-996.

90. Seimenis A. Overview of the epidemiological situation on echinococcosis in the Mediterranean region. Acta Trop 2003; 85: 191-195.

91. Menezes da Silva AM. Human echinococcosis: a neglected disease. Gastroenterol Res Pract 2010; pii: 583297.

92. Buttenschoen K, Carli Buttenschoen D. Echinococcus granulosus infection: the challenge of surgical treatment. Langenbecks Arch Surg 2003; 388: 218-230.

93. Khuroo MS, Dar MY, Yattoo GN, et al. Percutaneous drainage versus albendazole therapy in hepatic hydatidosis: a prospective, randomized study. Gastroenterology 1993; 104: 1452-1459.

94. Dervenis C, Delis S, Avgerinos C, et al. Changing concepts in the management of liver hydatid disease. J Gastrointest Surg 2005; 9: 869-877.

95. Guidelines for treatment of cystic and alveolar echinococcosis in humans. WHO Informal Working Group on Echinococcosis. Bull World Health Organ 1996; 74: 231-242.

96. Mueller L, Broering DC, Vashist $Y$, et al. A retrospective study comparing the different surgical procedures for the treatment of hydatid disease of the liver. Dig Surg 2003; 20: 279-284.

97. Stoot JH, Jongsma CK, Limantoro I, et al. More than 25 years of surgical treatment of hydatid cysts in a nonendemic area using the 'frozen seal' method. World J Surg 2010; 34: 106-113.

98. Saidi F, Nazarian I. Surgical treatment of hydatid cysts by freezing of cyst wall and instillation of 0.5 per cent silver nitrate solution. N Engl J Med 1971; 284: 1346-1350.

99. Reddick EJ, Olsen DO. Laparoscopic laser cholecystectomy. A comparison with mini-lap cholecystectomy. Surg Endosc 1989; 3: 131-133.

100. Dubois F, Berthelot G, Levard H. Laparoscopic cholecystectomy: historic perspective and personal experience. Surg Laparosc Endosc 1991; 1: 52-57.

101. Dagher I, Proske JM, Carloni A, et al. Laparoscopic liver resection: results for 70 patients. Surg Endosc 2007; 21: 619-624.

102. Descottes B, Glineur D, Lachachi F, et al. Laparoscopic liver resection of benign liver tumors. Surg Endosc 2003; 17: 23-30.

103. Farges $\mathrm{O}$, Jagot $\mathrm{P}$, Kirstetter $\mathrm{P}$, et al. Prospective assessment of the safety and benefit of laparoscopic liver resections. J Hepatobiliary Pancreat Surg 2002; 9: 242-248.

104. Morino M, Morra I, Rosso E, et al. Laparoscopic vs open hepatic resection: a comparative study. Surg Endosc 2003; 17: 1914-1918.

105. Simillis C, Constantinides VA, Tekkis PP, et al. Laparoscopic versus open hepatic resections for benign and malignant neoplasms--a meta-analysis. Surgery 2007; 141: 203-211.

106. Dubois F, Icard P, Berthelot G, Levard H. Coelioscopic cholecystectomy. Preliminary report of 36 cases. Ann Surg 1990; 211:60-62

107. Kelling G. Ueber Oesophagoskopie, Gastroskopie und Kölioskopie. Münch Med Wochenschr 1902; 49: 21-24.

108. Jacobeus H. Ueber die Möglichkeit die Zystoskopie bei Untersuchung seröser Höhlungen anzuwenden. Münch Med Wochenschr 1910; 57: 2090-2092.

109. Jacobeus H. Kurze Uebersichtüber meine Erfahrungen mit der Laparo-thoraskopie. Münch Med Wochenschr 1911; 58: 2017-2019. 
110. Cuesta MA, Meijer S, Paul MA, et al. Limited laparoscopic liver resection of benign tumors guided by laparoscopic ultrasonography: report of two cases. Surg Laparosc Endosc 1995; 5: 396-401.

111. Azagra JS, Goergen M, Gilbart E, et al. Laparoscopic anatomical (hepatic) left lateral segmentectomy-technical aspects. Surg Endosc 1996; 10: 758-761.

112. Cherqui D, Husson E, Hammoud R, et al. Laparoscopic liver resections: a feasibility study in 30 patients. Ann Surg 2000; 232: 753-762.

113. Cherqui D. Laparoscopic liver resection. Br J Surg 2003; 90: 644-646.

114. Dagher I, Belli G, Fantini C, et al. Laparoscopic hepatectomy for hepatocellular carcinoma: a European experience. J Am Coll Surg 2010; 211: 16-23.

115. Gigot JF, Glineur D, Santiago Azagra J, et al. Laparoscopic liver resection for malignant liver tumors: preliminary results of a multicentre European study. Ann Surg 2002; 236: 90-97.

116. Buell JF, Thomas MJ, Doty TC, et al. An initial experience and evolution of laparoscopic hepatic resectional surgery. Surgery 2004; 136: 804-811.

117. Chang S, Laurent A, Tayar C, et al. Laparoscopy as a routine approach for left lateral sectionectomy. Br J Surg 2007; 94: 58-63.

118. Buell JF, Cherqui D, Geller DA, et al. The international position on laparoscopic liver surgery: The Louisville Statement, 2008. Ann Surg 2009; 250: 825-830.

119. Braga M, Frasson M, Vignali A, et al. Laparoscopic vs. open colectomy in cancer patients: long-term complications, quality of life, and survival. Dis Colon Rectum 2005; 48: 2217-2223.

120. Murray A, Lourenco T, de Verteuil R, et al. Clinical effectiveness and cost-effectiveness of laparoscopic surgery for colorectal cancer: systematic reviews and economic evaluation. Health Technol Assess 2006; 10: 1-141, iii-iv.

121. McCormack K, Scott NW, Go PM, et al. Laparoscopic techniques versus open techniques for inguinal hernia repair. Cochrane Database Syst Rev 2003: CD001785.

122. van Dam RM, Hendry PO, Coolsen MM, et al. Initial experience with a multimodal enhanced recovery programme in patients undergoing liver resection. Br J Surg 2008; 95: 969-975.

123. Wind J, Polle SW, Fung Kon Jin PH, et al. Systematic review of enhanced recovery programmes in colonic surgery. Br J Surg 2006; 93: 800-809.

124. Fearon KC, Ljungqvist $\mathrm{O}$, Von Meyenfeldt $\mathrm{M}$, et al. Enhanced recovery after surgery: a consensus review of clinical care for patients undergoing colonic resection. Clin Nutr 2005; 24: 466-477.

125. Kehlet H, Wilmore DW. Multimodal strategies to improve surgical outcome. Am J Surg 2002; 183 : 630-641.

126. Wilmore DW, Kehlet H. Management of patients in fast track surgery. Bmj 2001; 322: 473-476.

127. Basse L, Madsen JL, Kehlet $\mathrm{H}$. Normal gastrointestinal transit after colonic resection using epidural analgesia, enforced oral nutrition and laxative. Br J Surg 2001; 88: 1498-1500.

128. Basse L, Raskov HH, Hjort Jakobsen $\mathrm{D}$, et al. Accelerated postoperative recovery programme after colonic resection improves physical performance, pulmonary function and body composition. $\mathrm{Br} \mathrm{J}$ Surg 2002; 89: 446-453.

129. Delaney CP, Zutshi M, Senagore AJ, et al. Prospective, randomized, controlled trial between a pathway of controlled rehabilitation with early ambulation and diet and traditional postoperative care after laparotomy and intestinal resection. Dis Colon Rectum 2003; 46: 851-859.

130. Zutshi M, Delaney CP, Senagore AJ, et al. Randomized controlled trial comparing the controlled rehabilitation with early ambulation and diet pathway versus the controlled rehabilitation with early ambulation and diet with preemptive epidural anesthesia/analgesia after laparotomy and intestinal resection. Am J Surg 2005; 189: 268-272.

131. Podore PC, Throop EB. Infrarenal aortic surgery with a 3-day hospital stay: A report on success with a clinical pathway. J Vasc Surg 1999; 29: 787-792.

132. Trondsen E, Mjaland O, Raeder J, et al. Day-case laparoscopic fundoplication for gastrooesophageal reflux disease. Br J Surg 2000; 87: 1708-1711.

133. Basse L, Hjort Jakobsen D, Billesbolle $P$, et al. A clinical pathway to accelerate recovery after colonic resection. Ann Surg 2000; 232: 51-57. 


\section{Chapter 1}

134. Schoetz DJ, Jr., Bockler M, Rosenblatt MS, et al. 'Ideal' length of stay after colectomy: whose ideal? Dis Colon Rectum 1997; 40: 806-810.

135. Kehlet $\mathrm{H}$. Multimodal approach to control postoperative pathophysiology and rehabilitation. $\mathrm{Br} \mathrm{J}$ Anaesth 1997; 78: 606-617.

136. Kehlet H, Dahl JB. Anaesthesia, surgery, and challenges in postoperative recovery. Lancet 2003; 362: 1921-1928.

137. Nygren J, Hausel J, Kehlet $\mathrm{H}$, et al. A comparison in five European Centres of case mix, clinical management and outcomes following either conventional or fast-track perioperative care in colorectal surgery. Clin Nutr 2005; 24: 455-461.

138. Spelt L, Ansari D, Sturesson C, et al. Fast-track programmes for hepatopancreatic resections: where do we stand? HPB (Oxford) 2011; 13: 833-838.

139. Adam R. Chemotherapy and surgery: new perspectives on the treatment of unresectable liver metastases. Ann Oncol 2003; 14 Suppl 2: ii13-16.

140. Abdalla EK, Adam R, Bilchik AJ, et al. Improving resectability of hepatic colorectal metastases: expert consensus statement. Ann Surg Oncol 2006; 13: 1271-1280.

141. Adam R, Delvart V, Pascal G, et al. Rescue surgery for unresectable colorectal liver metastases downstaged by chemotherapy: a model to predict long-term survival. Ann Surg 2004; 240: 644657; discussion 657-648.

142. Yeo SG, Kim DY, Kim TH, et al. Whole-liver radiotherapy for end-stage colorectal cancer patients with massive liver metastases and advanced hepatic dysfunction. Radiat Oncol 2010; 5: 97.

143. Krishnan S, Lin EH, Gunn GB, et al. Conformal radiotherapy of the dominant liver metastases: a viable strategy for treatment of unresectable chemotherapy refractory colorectal cancer liver metastases. Am J Clin Oncol 2006; 29: 562-567.

144. Schefter TE, Kavanagh BD. Radiation therapy for liver metastases. Semin Radiat Oncol 2011; 21: 264-270.

145. Andolino DL, Johnson CS, Maluccio M, et al. Stereotactic body radiotherapy for primary hepatocellular carcinoma. Int J Radiat Oncol Biol Phys 2011; 81: e447-453.

146. Minn AY, Koong AC, Chang DT. Stereotactic body radiation therapy for gastrointestinal malignancies. Front Radiat Ther Oncol 2011; 43: 412-427.

147. Chang DT, Swaminath A, Kozak M, et al. Stereotactic body radiotherapy for colorectal liver metastases: a pooled analysis. Cancer 2011; 117: 4060-4069.

148. Saxena A, Chua TC, Bester L, et al. Factors predicting response and survival after yttrium-90 radioembolization of unresectable neuroendocrine tumor liver metastases: a critical appraisal of 48 cases. Ann Surg 2010; 251: 910-916.

149. Evans KA, Richardson MG, Pavlakis N, et al. Survival outcomes of a salvage patient population after radioembolization of hepatic metastases with yttrium-90 microspheres. J Vasc Interv Radiol 2010; 21: 1521-1526.

150. Jakobs TF, Hoffmann RT, Dehm K, et al. Hepatic yttrium-90 radioembolization of chemotherapyrefractory colorectal cancer liver metastases. J Vasc Interv Radiol 2008; 19: 1187-1195.

151. Smits ML, Nijsen JF, van den Bosch MA, et al. Holmium-166 radioembolization for the treatment of patients with liver metastases: design of the phase I HEPAR trial. J Exp Clin Cancer Res 2010; 29: 70.

152. Mayo SC, Pawlik TM. Thermal ablative therapies for secondary hepatic malignancies. Cancer J 2010; 16: 111-117.

153. Jiao D, Qian L, Zhang Y, et al. Microwave ablation treatment of liver cancer with $2,450-\mathrm{MHz}$ cooledshaft antenna: an experimental and clinical study. J Cancer Res Clin Oncol 2010; 136: 1507-1516.

154. Bhardwaj N, Strickland AD, Ahmad F, et al. Microwave ablation for unresectable hepatic tumours: clinical results using a novel microwave probe and generator. Eur J Surg Oncol 2009; 36: 264-268.

155. Martin RC, Scoggins CR, McMasters KM. Safety and efficacy of microwave ablation of hepatic tumors: a prospective review of a 5-year experience. Ann Surg Oncol 2009; 17: 171-178. 
156. Nguyen KT, Laurent A, Dagher I, et al. Minimally invasive liver resection for metastatic colorectal cancer: a multi-institutional, international report of safety, feasibility, and early outcomes. Ann Surg 2009; 250: 842-848.

157. Stoot JH, van Dam RM, Busch OR, et al. The effect of a multimodal fast-track programme on outcomes in laparoscopic liver surgery: a multicentre pilot study. HPB (Oxford) 2009; 11: 140-144.

158. Kobayashi S, Nagano H, Marubashi S, et al. A single-incision laparoscopic hepatectomy for hepatocellular carcinoma: initial experience in a Japanese patient. Minim Invasive Ther Allied Technol 2010; 19: 367-371.

159. Gaujoux S, Kingham TP, Jarnagin WR, et al. Single-incision laparoscopic liver resection. Surg Endosc 2010; 25: 1489-1494.

160. Patel AG, Belgaumkar AP, James J, et al. Video. Single-incision laparoscopic left lateral segmentectomy of colorectal liver metastases. Surg Endosc 2010; 25: 649-650.

161. Giulianotti PC, Coratti A, Sbrana F, et al. Robotic liver surgery: results for 70 resections. Surgery 2010; 149: 29-39.

162. Jain G, Parmar J, Mohammed MM, et al. "Stretching the limits of laparoscopic surgery": two-stage laparoscopic liver resection. J Laparoendosc Adv Surg Tech A 2010; 20: 51-54.

163. Machado MA, Makdissi FF, Surjan RC, et al. Two-stage laparoscopic liver resection for bilateral colorectal liver metastases. Surg Endosc 2010; 24: 2044-2047.

164. SchindI MJ, Redhead DN, Fearon KC, et al. The value of residual liver volume as a predictor of hepatic dysfunction and infection after major liver resection. Gut 2005; 54: 289-296.

165. Shoup M, Gonen M, D'Angelica M, et al. Volumetric analysis predicts hepatic dysfunction in patients undergoing major liver resection. J Gastrointest Surg 2003; 7: 325-330.

166. Shah SA, Haddad R, Al-Sukhni W, et al. Surgical resection of hepatic and pulmonary metastases from colorectal carcinoma. J Am Coll Surg 2006; 202: 468-475.

167. Fusai G, Davidson BR. Management of colorectal liver metastases. Colorectal Dis 2003; 5: 2-23.

168. Scheele J, Altendorf-Hofmann A, Grube T, et al. [Resection of colorectal liver metastases. What prognostic factors determine patient selection?]. Chirurg 2001; 72: 547-560.

169. Karlo C, Reiner CS, Stolzmann P, et al. CT- and MRI-based volumetry of resected liver specimen: comparison to intraoperative volume and weight measurements and calculation of conversion factors. Eur J Radiol 2010; 75: e107-111.

170. Dello SA, van Dam RM, Slangen JJ, et al. Liver volumetry plug and play: do it yourself with ImageJ. World J Surg 2007; 31: 2215-2221.

171. van der Vorst JR, van Dam RM, van Stiphout RS, et al. Virtual liver resection and volumetric analysis of the future liver remnant using open source image processing software. World J Surg 2010; 34: 2426-2433.

172. Alkari B, Owera A, Ammori BJ. Laparoscopic liver resection: preliminary results from a UK centre. Surg Endosc 2008; 22: 2201-2207.

173. Stoot JH, van Roosmalen J, Terpstra OT, et al. Life-threatening hemorrhage from adenomas in the liver during pregnancy. Dig Surg 2006; 23: 155.

174. Huurman VA, Stoot JH, van der Linden E, et al. Necrosis of a large hepatic tumor after hemorrhage and subsequent selective arterial embolization. World J Gastroenterol 2006; 12: 6059-6061.

175. Stoot JH, van der Linden E, Terpstra OT, et al. Life-saving therapy for haemorrhaging liver adenomas using selective arterial embolization. Br J Surg 2007; 94: 1249-1253.

176. Eckhauser FE, Knol JA, Raper SE, et al. Enucleation combined with hepatic vascular exclusion is a safe and effective alternative to hepatic resection for liver cell adenoma. Am Surg 1994; 60: 466471; discussion 472.

177. Cobey FC, Salem RR. A review of liver masses in pregnancy and a proposed algorithm for their diagnosis and management. Am J Surg 2004; 187: 181-191. 



\section{Part 2}

Surgical management of benign cystic liver lesions: hydatid disease 
Part 2 


\section{INTRODUCTION}

Echinococcosis or hydatid disease of the liver is caused by a zoonotic infection from predominantly the larval forms of the tapeworm Echinococcus granulosus. ${ }^{1}$ Echinococcosis occurs in endemic large, sheep-raising areas. ${ }^{2-7}$ In Western industrial nations, the incidence of hydatid disease is relatively low. ${ }^{5,8,9}$ As immigration and travelling became more common, the prevalence of the disease increased in Europe and North America. ${ }^{3,8,10}$ In the Netherlands it is almost always an imported disease.

The tapeworm is found in the small intestines of dogs, the definitive hosts. The tapeworm eggs are passed in the faeces and thereby contaminate herbage and are consequently ingested by intermediate hosts, including mainly sheep, but also yaks, goats and camels. Humans can be incidentally infected by ingestion of the parasite eggs transmitted through consumption of contaminated unwashed vegetables and contact with infected animals. After hatching in the small intestine and penetrating the gut wall, the eggs reach the organs after passage through the portal vein. In these organs they develop into a hydatid cyst. The most frequent site for cystic lesions is the liver, followed by the lungs. In fact, infection can affect all tissues in the human body. ${ }^{4,6-9,11,12}$

To investigate the presentation, treatment and outcome of surgically treated patients in the Netherlands, first a single centre study from the Harbour hospital, centre for Tropical diseases, was performed.

Interestingly, an extremely rare case was noted in the south of the Netherlands, which strengthen the quest for answers for this rare, benign disease.

After performing the study in Rotterdam, a decreasing number in operative procedures for hydatid disease of the liver was observed. Therefore, it was decided to investigate whether the treatment shifted from open resection towards less invasive procedures (PAIR), or was caused by better medication, or simply less infected patients?

In this part the following questions were investigated:

1. Is a technique invented in the seventies of the former century still effective and safe in non-endemic areas in the minimal invasive era?

2. How is this rare disease treated and is there any treatment trend towards less invasive procedures?

3. Is the incidence of hydatid disease of the liver in the Netherlands decreasing? 


\section{REFERENCES}

1. Brunetti E, Kern P, Vuitton DA. Expert consensus for the diagnosis and treatment of cystic and alveolar echinococcosis in humans Acta Trop 2010; 114: 1-16.

2. Dziri $C$, Haouet $K$, Fingerhut A. Treatment of hydatid cyst of the liver: where is the evidence? World J Surg 2004; 28: 731-736.

3. Sayek I, Tirnaksiz MB, Dogan R. Cystic hydatid disease: current trends in diagnosis and management Surg Today 2004: 34: 987-996.

4. Yagci G, Ustunsoz B, Kaymakcioglu N, et al. Results of surgical, laparoscopic, and percutaneous treatment for hydatid disease of the liver: 10 years experience with 355 patients World J Surg 2005; 29: 1670-1679.

5. Smego RA, Jr., Bhatti S, Khaliq AA, et al. Percutaneous aspiration-injection-reaspiration drainage plus albendazole or mebendazole for hepatic cystic echinococcosis: a meta-analysis Clin Infect Dis 2003; 37: 1073-1083.

6. Khuroo MS, Wani NA, Javid G, et al. Percutaneous drainage compared with surgery for hepatic hydatid cysts N Engl J Med 1997; 337: 881-887.

7. Gourgiotis S, Stratopoulos C, Moustafellos P, et al. Surgical techniques and treatment for hepatic hydatid cysts Surg Today 2007; 37: 389-395.

8. Buttenschoen K, Carli Buttenschoen D. Echinococcus granulosus infection: the challenge of surgical treatment Langenbecks Arch Surg 2003; 388: 218-230.

9. Smego RA, Jr., Sebanego P. Treatment options for hepatic cystic echinococcosis Int J Infect Dis 2005; 9: 69-76.

10. Khuroo MS, Dar MY, Yattoo GN, et al. Percutaneous drainage versus albendazole therapy in hepatic hydatidosis: a prospective, randomized study Gastroenterology 1993; 104: 1452-1459.

11. Dervenis C, Delis S, Avgerinos C, et al. Changing concepts in the management of liver hydatid disease J Gastrointest Surg 2005; 9: 869-877.

12. Guidelines for treatment of cystic and alveolar echinococcosis in humans. WHO Informal Working Group on Echinococcosis Bull World Health Organ 1996; 74: 231-242. 


\section{Chapter 2}

The first locally-acquired human infection of Echinococcus multilocularis in the Netherlands

L. van Dommelen, J.H.M.B. Stoot, V.C. Cappendijk, M.A. Abdul Hamid, F.F. Stelma, L.M. Kortbeek, J. van der Giessen and A.M.L. Oude Lashof

Adapted from J Clin Microbiol. 2012;50(5):1818-20

Presented to the European Society of Clinical Microbiology and Infectious Diseases in Vienna, Austria, April 2010. 
Chapter 2

\section{ABSTRACT}

In the northern part of Western Europe, Echinococcus multilocularis is primarily detected and spreading in foxes. The present case marks E. multilocularis as an emerging pathogen for humans, as it describes the first human case of probably locally acquired E. multilocularis in the Netherlands, with various interesting clinical aspects. 


\section{CASE REPORT}

A 55-year-old female presented at our hospital with cervical pain. She had a history of myxoid liposarcoma in the left upper leg (1993) and metastases in the spinal column (2001 and 2007). As new metastases in the cervical spinal column were suspected, diagnostic work-up was initiated. Fluorodeoxyglucose positron emission tomography (FDG-PET) was negative, but computed tomography (CT), simultaneously performed with FDG-PET, and magnetic resonance imaging (MRI) showed seven lesions in the liver, with a maximum size of $1.7 \mathrm{~cm}$ (Figure 1). These lesions were not present in an abdominal CT performed 6 months earlier. The patients' laboratory results were as follows: alkaline phosphatase $89 \mathrm{U} / \mathrm{l}$, gamma-glutamyl transferase $28 \mathrm{U} / \mathrm{I}$, aspartate aminotransferase $19 \mathrm{U} / \mathrm{I}$, alanine aminotransferase 23 $\mathrm{U} / \mathrm{I}$, lactate dehydrogenase $346 \mathrm{U} / \mathrm{l}$, bilirubin $10.1 \mathrm{micromol} / \mathrm{I}$ and white blood cells $4.9 \times 10^{9} / \mathrm{L}$ with $62 \%$ neutrophils, $22 \%$ lymfocytes, $15 \%$ monocytes, $1 \%$ eosinophils and $0 \%$ basophils.

Without pathological confirmation of these presumed metastases of her myxoid lipsarcoma, neo-adjuvant chemotherapy was started. As this was bilobar liver disease, radical surgery with curative intent was only possible when performing two separate operations in order to prevent liver failure. Therefore, after two chemotherapy cycles, partial left hepatectomy and contra-lateral portal ligation was performed, to allow liver regeneration, leaving four lesions in situ in the right liver lobe. These were to be operated after the enlargement of the liver remnant.

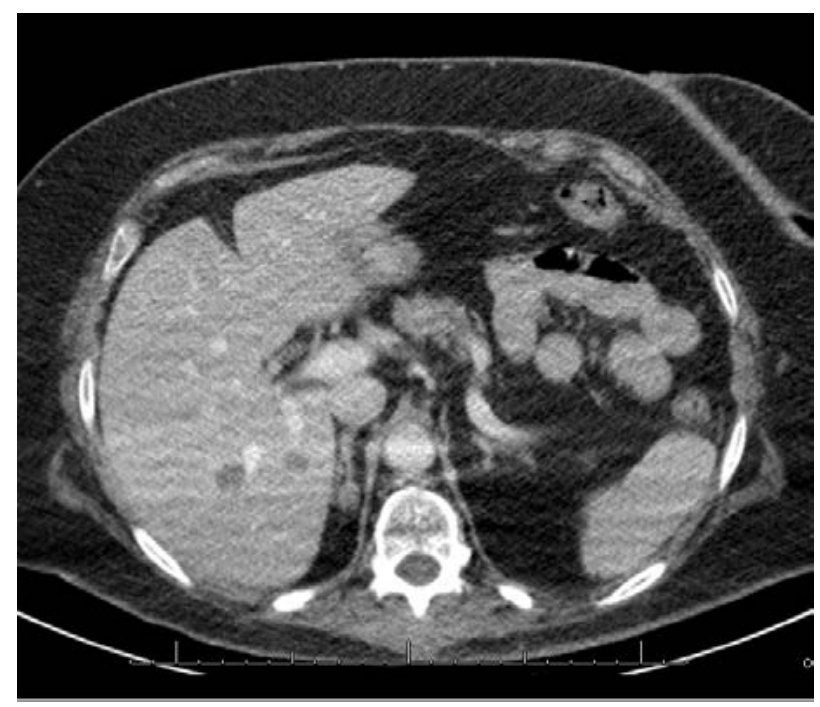

Figure 1. Computed tomogram of the liver post intravenously iodine containing contrast, scanned in portovenous phase. In this transverse CT image of the liver, two hypodens lesions are seen, suspect for metastases 
Pathologic examination of the liver tissue revealed three circumscribed intact nodules with central necrosis and a peripheral wall of histiocytes with focal giant cells. Central cysts and budding daughtercysts with a trilayered membrane wall were seen, suiting echinococcosis. No protoscolices, hooklets or calcareous corpuscles were noticed. Protoscolices, however, are often absent in E. multilocularis lesions. ${ }^{1}$ Hence the findings were compatible with echinococcosis (Figure 2 and 3). There was no evidence of metastases of the liposarcoma. Subsequently, serum of this patient was sent to a Dutch reference centre and Echinococcus granulosus serology came back weakly positive (IgG 1:80 (in-house enzyme-linked immunosorbent assay, cut-of 1:40), immunoblot IgG1 positive, IgG4 negative (in-house immunoblot). ${ }^{2}$ The result of the specific Echinococcus multilocularis ELISA (Em2plus) was negative.

To finally confirm the diagnosis, isolated DNA from unpreserved liver material was sent to the Centre for Infectious Disease Control (Bilthoven, Netherlands). DNA from our patients' lesions was compared with DNA isolated from E. multilocularis samples from infected foxes originating from Limburg province (The Netherlands); this was the province where the patient lived. A polymerase chain reaction (PCR) amplifying DNA sequences of two mitochondrial targets was used: cytochrome $c$ oxidase subunit 1 (COX 1) and NADH dehydrogenase (nad1) to identify E. multilocularis and to distinguish the organism from Echinococcus granulosus and other taeniid tapeworms. ${ }^{3,4}$ The DNA sequences were compared to DNA sequences from the same target from other E. multilocularis and E. granulosus strains in Genbank. DNA sequencing of the PCR amplicons of the nad1 (520bp) and cox1 (420bp) genes showed a $99-100 \%$ similarity with the European E. multilocularis strains, including four E. multilocularis fox strains, and only a $86-93 \%$ similarity with E. granulosus. After this diagnosis, our patient started with albendazole $400 \mathrm{mg}$ twice daily. Subsequent abdominal CT-scans, 4 and 7 months post-resection, revealed no progression of the lesions nor a change in aspect. Informed consent was obtained and this report was approved by the human investigations committee of the Maastricht University Medical Centre. 


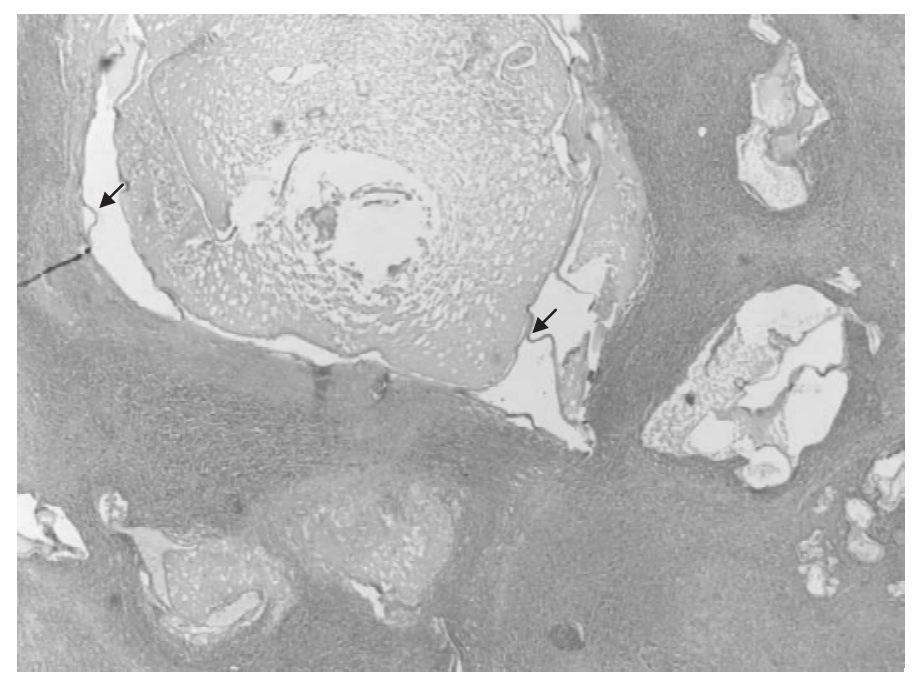

Figure 2. Histopathology of liver lesion (100x, H\&E stain). Captured foreign material (arrows) suspect for Echinococcus sp.

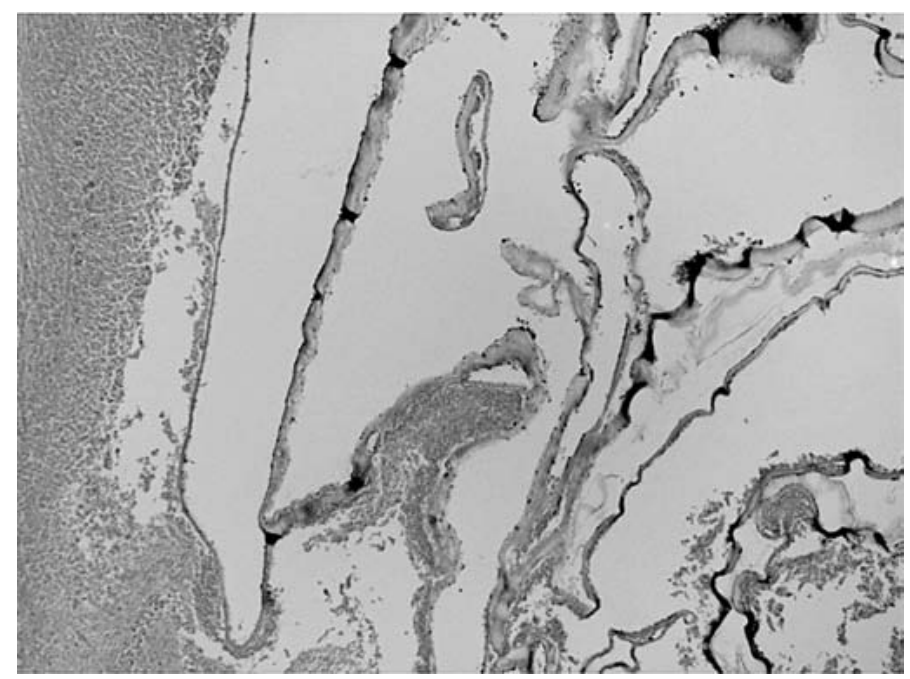

Figure 3. Histopathology of liver lesion (400x, H\&E stain). Separated membranes in a cyst wall with peripheral necrosis.

\section{DISCUSSION}

Four lessons can be learned from this case presentation. Firstly, E. multilocularis lesions can be rapidly progressive: multiple lesions with a maximum size of $1.7 \mathrm{~cm}$ developed within a period of 6 months in our patient. The incubation period after ingestion is estimated to be between 5 to 15 years. ${ }^{1}$ Liver cysts grow up to $1 \mathrm{~cm}$ in 
the first six months and $2-3 \mathrm{~cm}$ per year thereafter, but in our patient one lesion was already $1.7 \mathrm{~cm}$ in less than 6 months time. ${ }^{5}$ When left untreated, the mortality rate is almost $100 \%$ within 15 years after diagnosis. ${ }^{1}$ Recently, a very progressive Echinococcus multilocularis infection has been described in a renal transplant patient who was severely immunocompromised. ${ }^{6}$ Our patient however did not use immunosuppressive drugs in the period before presentation.

Secondly, E. multilocularis lesions can be misinterpreted for metastatic lesions, as E. multilocularis lesions usually present on CT with irregular borders and various densities, although variation exist. ${ }^{7}$ Calcifications are present in $82 \%$ and are hyperdense, while necrotic areas are hypodense. "Hot spots" are often seen with FDG-PET, although lesions can be metabolically inactive as well, as in our case. ${ }^{7-9}$ The FDG-PET-negative, multiple circumscript hypodense lesions in the liver seen at regular CT in our patient were not classical for E. multilocularis and did resemble liver metastases; ${ }^{10}$ the initial presumptive diagnosis therefore seemed much more likely.

Thirdly, E. multilocularis lesions can develop in patients without reported behavior associated with the ingestion of E. multilocularis eggs. ${ }^{11}$ Our patients lifetime travel history revealed three short holidays to endemic areas (Switzerland, Italy and Austria in 2006 and 2007) ${ }^{12}$, but she had never lived in an endemic area. She was not extensively exposed to forest environment, did not hunt, did not consume forest fruits, had no contact with (domestic) animals, did not work in her garden and bought her fruits and vegetables in regular supermarkets.

Lastly, serological results do not necessarily correspond with disease. E. multilocularis is known to cross-react with $E$. granulosus in serological assays ${ }^{1}$ and $E$. granulosus assays are therefore used in the E. multilocularis work-up. Serology results in our patient were weakly positive, illustrating that imaging is essential to exclude $E$. multilocularis infection. We do not have an explanation for the weak serology results; it might be due to a difference in the $E$. multilocularis strain in our patient. There is no serological evidence of widespread human contact with $E$. multilocularis in the Netherlands yet, ${ }^{13}$ but these results should be interpreted with caution. Although E. multilocularis is prevalent in foxes in the southern part of the Netherlands, no human E. multilocularis case acquired in the Netherlands has been described so far. Recently three E. multilocularis cases have been described in a part of Belgium, which borders the southern part of the Netherlands, where our patient resides. ${ }^{14}$ Sequencing results could not define the exact geographical location of acquisition of the $E$. multilocularis strain in our patient but the Netherlands belongs to the possibilities. Although due to the long incubation period (5-15 years), the time and place of infection is hard to determine retrospectively, considering the duration of exposure in this patient, domestically-acquired E. multilocularis seems most likely. 
This patient represents the first reported case of Echinococcus multilocularis acquired in the Netherlands. Surveillance data originating from foxes in the southern part of the Netherlands have shown that E. multilocularis spreads by $2.7 \mathrm{~km}$ per year in a northern direction and based on the spreading of $E$. multilocularis in foxes in this area in the Netherlands ${ }^{14}$ the human risk in Limburg province is estimated to be three human cases by $2018 .{ }^{15}$ We therefore believe that E. multilocularis is an emerging pathogen in Western Europe and clinicians should consider this diagnosis when confronted with (asymptomatic) liver lesions.

\section{REFERENCES}

1. Eckert J, and Deplazes P. Biological, epidemiological, and clinical aspects of echinococcosis, a zoonosis of increasing concern. Clin Microbiol Rev 2004;17: 107-135.

2. Herremans T, Verweij JJ, Schipper HG, Casparie M, van Lieshout L, Pinelli E, et al. [Decline of echinococcosis in the Netherlands; 1997-2008]. Ned Tijdschr Geneeskd 2010; 154: A2297.

3. Bowles J, Blair D, and McManus DP. Genetic variants within the genus Echinococcus identified by mitochondrial DNA sequencing. Mol Biochem Parasitol 1992;54: 165-173.

4. Bowles J, and McManus DP. NADH dehydrogenase 1 gene sequences compared for species and strains of the genus Echinococcus. Int J Parasitol 1993;23: 969-972.

5. Pedrosa I, Saiz A, Arrazola J, Ferreiros J, and Pedrosa CS. Hydatid disease: radiologic and pathologic features and complications. Radiographics 2000;20:795-817.

6. Geyer M, Wilpert J, Wiech T, Theilacker C, Stubanus M, Kramer-Zucker A, et al. Rapidly progressive hepatic alveolar echinococcosis in an ABO-incompatible renal transplant recipient. Transpl Infect Dis. Transpl Infect Dis 2011;13:278-284

7. Ehrhardt AR, Reuter S, Buck AK, Haenle MM, Mason RA, Gabelmann A, et al. Assessment of disease activity in alveolar echinococcosis: a comparison of contrast enhanced ultrasound, three-phase helical CT and [(18)F] fluorodeoxyglucose positron emission tomography. Abdom Imaging 2007;32: 730-736

8. Bresson-Hadni S, Delabrousse E, Blagosklonov O, Bartholomot B, Koch S, Miguet JP, et al. Imaging aspects and non surgical interventional treatment in human alveolar echinococcosis. Parasitol Int 2006;55 Suppl: S267-272.

9. Reuter S, Schirrmeister H, Kratzer W, Dreweck C, Reske SN, and Kern P. Pericystic metabolic activity in alveolar echinococcosis: assessment and follow-up positron emission tomography. Clin Infect Dis 1999;29:1157-1163.

10. Sheah K, Ouellette HA, Torriani M, Nielsen GP, Kattapuram S, and Bredella MA. Metastatic myxoid liposarcomas: imaging and histopathologic findings. Skeletal Radiol 2008;37:251-258.

11. Kern P, Ammon A., Kron M, Sinn G, Sander S, Petersen LR, et al. Risk factors for alveolar echinococcosis in humans. Emerg Infect Dis 2004;10:2088-2093.

12. Romig T, Dinkel A, and Mackenstedt U. The present situation of echinococcosis in Europe. Parasitol Int 2006;55 Suppl:S187-191.

13. Kortbeek LM, Harms M, van Pelt W, van der Giessen J and Pinelli Ortiz E. Echinococcus multilocularis in the Netherlands: what about humans? ECCMID, Milan, Italy, 2011. P859.

14. Takumi K, de Vries A, Chu ML, Mulder J, Teunis P, and van der Giessen J. Evidence for an increasing presence of Echinococcus multilocularis in foxes in The Netherlands. Int J Parasitol 2008;38:571-8.

15. Takumi K, Hegglin D, Deplazes P, Gottstein B, Teunis P, and van der Giessen J. Mapping the increasing risk of human alveolar echinococcosis in Limburg, The Netherlands. Epidemiol Infect 2011; 1-5. 



\section{Chapter 3}

\section{Over 25 years of surgical treatment of hydatid cysts in a non-endemic area using the 'frozen seal' method}

J.H.M.B. Stoot, C.K. Jongsma, I. Limantoro, O.T. Terpstra, P.J. Breslau

Adapted from World J Surg. 2010; 34(1):106-13

Presented to the $8^{\text {th }}$ World Congress of the International Hepato-Pancreatico Biliary Association in Mumbai, India, February 2008. 


\section{ABSTRACT}

Background: Hydatid disease of the liver remains endemic in the world and is an imported disease in the Netherlands. The aim of this study was to evaluate treatment and outcome of surgically treated patients for hydatid disease in a single centre in the Netherlands.

Methods: This retrospective study included 112 consecutive patients surgically treated for hydatid disease between 1981 and 2007. Primary outcome was relapse of disease. Secondary outcomes were infections, complications, reoperations, length of hospital stay and mortality.

Results: In all cases, echinococcosis was diagnosed by computed tomography or ultrasound (US). Serology (ELISA, IMF) confirmed the diagnosis in 92.9\%. Most of the cysts were seen only in the liver (73.5\%). All cysts were operated on with the frozen seal technique. Relapse of disease was seen in $9(8.0 \%)$ cases. Five $(4.5 \%)$ required surgical treatment at a later stage. Twenty $(17.9 \%)$ complications were recorded. Four (3.6\%) needed radiological drainage and three (2.7\%) a reoperation. Follow up was performed with US and/or serology at a mean of 24 months (range 0.5-300 months). All but one complications were seen in the liveroperated group, this proved not to be of statistical significance $(p=0.477)$. Patients with complications stayed significantly longer in hospital than did the patients without complications $(p<0.001)$. No mortality was observed in this study.

Conclusion: The present study suggests that the frozen seal method of surgery for hydatid disease is safe and effective. Future studies are needed to prove its position in the treatment of hydatid disease as new developments show promising results. 


\section{INTRODUCTION}

Hydatid cyst disease, or human cystic echinococcosis, is a zoonotic infection mainly caused by the larval forms of the tapeworm Echinococcus granulosus. ${ }^{1-8}$ Echinococcosis occurs worldwide in endemic large sheep-raising areas including Africa, the Mediterranean region of Europe, the Middle East, Asia, South America, Australia and New Zealand. ${ }^{2,3,5-10}$ The incidence of hydatid disease in Western industrial nations is relatively low. ${ }^{6,8,10-11}$ However, the prevalence of the disease has increased in Europe and North America due to immigration and travelling. ${ }^{5,12}$ In the Netherlands it is almost always an imported disease. ${ }^{13,14}$ The most frequent site for cystic lesions is the liver ( $60 \%$ of cases), followed by the lungs (about $20 \%$ of cases). In fact, infection can affect all tissues in the human body. $1,3,6-8,10,11,15$

There are three treatment options for hepatic cystic echinococcosis: chemotherapy, surgery and percutaneous drainage (puncture, aspiration, injection, reaspiration-also known as PAIR) or a combination of these therapies. ${ }^{6-8}$ Although PAIR has been investigated by several groups, there is still no consensus about the best treatment. Surgery may vary from conservative (cystectomy) to radical (complete open resection) to laparoscopic techniques. The debate on the best surgical approach is still ongoing, whether it is conservative surgery or radical surgery in which the cyst is totally removed including the pericyst by total pericystectomy or partial hepatectomy. ${ }^{16}$

In an area where hydatid disease is non-endemic and therefore not a routine treatment, it is of major importance to perform a safe, reproducible technique with a low complication rate. The frozen seal method as surgical treatment for hydatid disease, described by Saidi and Nazarian during 1970's, has been used in the Harbour Hospital since $1981 .^{17}$

The aim of this study was to investigate the results of the frozen seal method as treatment of echinococcosis in a specialized centre in the Netherlands over the past 25 years.

\section{METHODS}

This study included all consecutive surgically treated patients in the Harbour Hospital in Rotterdam, the Netherlands, between 1981 and 2007. All medical records were reviewed concerning the following parameters: age, sex, country of origin, presentation of symptoms, location and type of cysts diagnosed by radiologic imaging, treatment, hospital stay and outcomes. Radiologic workup included conventional radiology, ultrasonography (US) and computed tomography (CT). Laboratory tests and serology were performed to confirm the diagnosis. Enzyme-linked immunosorbent assay (ELISA), Immunoelectroforesis (IE), and in most cases the comple- 
ment binding reaction (CBR) were used as serologic testing for diagnosis and follow-up.

Primary outcome was relapse of disease. Secondary outcomes were infections, complications, spilling of hydatid cyst content, reoperations, length of stay and mortality.

An echinococcal cyst was diagnosed if any two of the features were present: 1) typical sonographic and CT appearance demonstrating multilocular cystic lesion with daughter cysts, calcification of the cysts walls, or both; 2) positive indirect hemagglutination test or ELISA for echinococcal infection; and 3) histology demonstrating a parasite-derived laminated membrane with or without viable scolices. ${ }^{4,11,18}$ Indications for surgery were the presence of pain, jaundice, fever, dyspnea or cardiac decompensation. The World Health Organisation (WHO) indications for surgery were respected in all cases: large liver cysts with multiple daughter cysts, infected cysts and cysts communicating with the biliary tree. Moreover, surgery was considered indicated in the presence of cysts in the lung, heart, and other organs. $^{11,15}$

Prior to the operation in some cases [ $n=28,25.0 \%$ ] albendazole (400mg twice a day) was administered and continued 3 months after surgery. Before 1988, mebendazole was used in selected cases [ $n=19,17.0 \%]$. From 1990, albendazole was given depending on the patient's clinical situation (severe pain, fever, other clinical signs of echinococcosis infection). From 1995, the regimen was standardized, and almost all patients received preoperative drug treatment (14/15 patients). One patient received Eskazole (albendazole).

\section{Surgical technique}

In this study, the frozen seal technique was used for open surgery in all cases. For hydatid cysts in the lung or myocardium, the thorax was opened via median sternotomy incision. For liver cysts, the abdomen was exposed through a bilateral subcostal incision, abdominal viscera were examined for potential sites of dissemination, and the liver was mobilized. Using this technique, a $7 \mathrm{~cm}$ long metal cone with a large $(8 \mathrm{~cm}$ diameter) upper and $4 \mathrm{~cm}$ lower aperture was used. It has a fine tubing soldered to the rim of the lower opening. A refrigerant is circulated via the inlet of the tubing. In this setting, Freon was used in all cases as refrigerant. The ringshaped tubing of the cone was frozen onto the cyst on the surface of the liver (or lung), producing a watertight frozen seal. The contents of the cysts could then be evacuated through the cone without risk of uncontrolled rupture or spilling. ${ }^{13,17,19}$ Normally the fluid is crystal clear. If the fluid was turbid, a communication with bile ducts or secondary infection was suspected, and material for bacteriologic examination was obtained (and, consequently, antibiotic treatment was started). 
Then the membrane was excised completely and the residual space was meticulously inspected for biliary communication. If there was any suspicion on biliary communication, fistulas were closed with absorbable sutures. If no communication was present, the residual cavity was flushed for 2 min with a freshly prepared $0.5 \%$ silver nitrate solution to kill any remaining scolices. The use of this scolicidal agent was based on the evidence provided by Saidi and Nazarian. ${ }^{17,20}$ Silver nitrate $0.5 \%$ solution proved to be a suitable alternative for formalin in a mouse model with standardized intraperitoneal inoculation techniques. It effectively sterilized scolices within 2 min and, more importantly, completely non-toxic for other tissues. Clinical application of silver nitrate $0,5 \%$ led to effective treatment without any local or systemic effect of the silver nitrate washout in more than 55 surgically treated patients with liver and lung hydatid cysts. ${ }^{20}$

After the use of silver nitrate solution, crypts or recesses were carefully checked for residual cyst material. If present, this was removed and a second treatment with silver nitrate solution was administered.

Again, the residual cavity was meticulously inspected for biliary communication. Any visible fistulas were sutured with absorbable sutures. If the residual space communicated with bile ducts or bronchus, any contact between the silver nitrate solution and these structures was avoided either by compression of the liver or by increasing the end- expiratory pressure of lung ventilation. No residual space should be present after this procedure as it probably enhances the possibility of infection. Therefore, the cavity was filled with hypertonic saline and closed with non-absorbable sutures or, in selected cases, obliteration with greater omentum was performed.

If appropriate, cavities were drained with $18 \mathrm{~F}$ vacuum tubes, and an additional subhepatic drain was placed in selected cases to control any possible biliary leakage. Drains were removed on the fifth to seventh postoperative day. Patients who required prolonged drainage were considered to have biliary fistulas. For patients with symptoms of an obstructed or communicating common bile duct (CBD), intraoperative cholangiography and choledochotomy with T-tube drainage was performed. In selected cases, endoscopic retrograde cholangiopancreatography (ERCP) was performed to drain the CBD.

\section{Follow-up}

Complications were defined as "every unwanted development in the illness of the patient or in the treatment of the patient's illness that occurs in the clinic". ${ }^{21}$ Complications were classified according to the recent grading system of Dindo et al (table 1). ${ }^{22}$ Spilling was considered as an error in technique, that could lead to a complication (infection) or relapse of disease. 


\section{Chapter 3}

Table 1. Classification of Surgical Complications

\begin{tabular}{|c|c|}
\hline Grade $^{a}$ & Definition \\
\hline Grade I & $\begin{array}{l}\text { Any deviation from the normal postoperative course without the need for pharmacological } \\
\text { treatment or surgical, endoscopic, and radiological interventions } \\
\text { Allowed therapeutic regimens are: drugs as antiemetics, antipyretics, analgetics, diuretics, } \\
\text { electrolytes, and physiotherapy. This grade also includes wound infections opened at the } \\
\text { bedside }\end{array}$ \\
\hline Grade II & $\begin{array}{l}\text { Requiring pharmacological treatment with drugs other than such allowed for grade I com- } \\
\text { plications } \\
\text { Blood transfusions and total parenteral nutrition are also included }\end{array}$ \\
\hline Grade III & Requiring surgical, endoscopic or radiological intervention \\
\hline Grade IIla & Intervention not under general anesthesia \\
\hline Grade IIIb & Intervention under general anesthesia \\
\hline Grade IV & Life-threatening complication (including CNS complications) ${ }^{b}$ requiring IC/ICU management \\
\hline Grade IVa & Single organ dysfunction (including dialysis) \\
\hline Grade IVb & Multiorgan dysfunction \\
\hline Grade V & Death of a patient \\
\hline
\end{tabular}

From Dindo et al. ${ }^{22}$

CNS central nervous system, IC intermediate care, ICU intensive care unit

a The suffix " $d$ " (for "disability") is added to the respective grade of complication if the patient suffers from a complication at the time of discharge. It indicates the need for a follow-up to evaluate the complication fully

brain haemorrhage, ischemic stroke, subarachnoidal bleeding, but excluding transient ischemic attacks.

After discharge all patients were followed periodically at the first, third, sixth and twelfth postoperative months during the first year and at least once a year thereafter. Seamen were unable to be followed long term. The follow-up procedure included physical examination, serologic tests, US of the abdomen, and CT scanning in particular cases. Relapse or recurrence of disease was defined as appearance of new extrahepatic disease resulting from procedure- related spilling or reappearance of live cysts at the site of a previously treated cyst. ${ }^{7,23}$

\section{Statistical analysis}

Categoric variables were presented as numbers (percentages) and continuous variables by the median and range. Complications were analyzed using Fisher's exact test. Hospital stay was analyzed using the Mann-Whitney $U$ - test. The analyses did not account for missing data. A value of $P<0.05$ was considered to be statistically significant. Data were analyzed using SPSS ${ }^{\circledR}$ version 13.0 for Windows ${ }^{\circledR}$ (SPSS, Chicago, IL, USA). 


\section{RESULTS}

The study describes a total of 112 consecutive patients who underwent surgery for hydatid disease during the years 1981 - 2007 at the Harbour Hospital in Rotterdam, The Netherlands. All but five patients were from abroad, either immigrants or seamen. Demographic data are presented in Table 2. At clinical presentation, the complaints consisted mainly of abdominal pain [ $n=87(77.7 \%)]$, abdominal mass [ $n$ $=43(38.4 \%)]$, fever $[n=20(17.9 \%)]$, and urticaria $[n=11(9.8 \%)]$ or a combination of these symptoms. Also, acute abdominal complaints due to rupture [ $=3(2.7 \%)$, cardiac decompensation $[n=2(1.8 \%)]$, anaphylaxis $[n=1(0.9 \%)]$ and jaundice $[n=$ $8(7.1 \%)]$ were seen at presentation. In all cases, echinococcosis was diagnosed by US and CT scanning. Serology [ELISA, immunofluorescence (IMF)] confirmed the diagnosis in $92.9 \%$ of the cases. Liver function tests were abnormal only when the hepatic ducts were involved.

Table 2. Demografic data of patients treated for hydatid disease in the Harbour Hospital: $1981-2007$

\begin{tabular}{lc}
\hline Age (median, range) & $38[7-70]$ years \\
Sex (M : F) & $52: 60$ \\
No. of patients, by country of origin & 1 \\
Armenia & 1 \\
India & 2 \\
Italy & 1 \\
Iraq & 1 \\
Lebanon & 1 \\
Libya & 37 \\
Morocco & 5 \\
Netherlands & 3 \\
Spain & 1 \\
Sudan & 52 \\
Turkey & 1 \\
Uruguay & 4 \\
Unknown & 2 \\
Yugoslavia & 112 \\
Total &
\end{tabular}

Most of the cysts were seen only in the liver, although other, more rare locations were found including the myocardium and the adductores muscles in the right leg. The locations are given in Table 3 together with the number of complications per organ. Surgery was performed in all cases [ $n=112]$ with the frozen seal method, and more than 196 cysts were operated in the liver, lungs and abdomen. Primary and secondary outcomes were scored in the clinic and after discharge during a median follow-up of 24 months (0.5 - 300 month) (Table 4). Twelve patients (10.7\%) were 
lost to follow-up. Relapse of disease was observed in nine patients (8.0\%) during a median follow-up period of 33 months(10-300 months). In this group with recurrence $(n=9), 6$ patients were initially treated before and after surgery with medication (mebendazole $n=2$, abendazole $n=4$, no medication $n=3$ ). Regarding further treatment four patients (3.6\%) needed five reoperations $(4.5 \%)$ in a later stage. The remaining five patients were treated successfully with albendazole (400mg twice a day).

Grade I complications ( $n=2)$ consisted of two wound infections. The moderate complications (grade II, $\mathrm{n}=10$ ) were two biliary fistulas, two pneumonias, two urinary tract infections, and four infections of the remnant cavity. More severe complications (grade III) with interventions consisted of fascia dehiscence, pleural effusion, and cavity infections and/or abscesses that required radiological drainage $(n=4)$ or relaparotomy $(n=3)$. One grade IV complication, acute respiratory distress syndrome (ARDS), occurred after percutaneous treatment of a hydatid cyst in the liver in segment 8 due to perforation and rupture to the lung. No complications or abnormal liver function tests were noted related to the use of silver nitrate $0.5 \%$ solution. No mortality was observed in this study.

Table 3. Number, location and largest diameter of hydatid cysts of patients surgically treated in the Harbour Hospital: 1981 - 2007. In some records the diameter and/or the numbers of cysts were not reported, but were regarded as multiple. These are not reported in this table*.

\begin{tabular}{lll}
\hline Organ & Number of cysts & Complications (number of patients) \\
\hline Liver* (no. of patients) & $144(73.5 \%)$ & $19(95.0 \%)$ \\
$\quad$ Grade I $(n=2)$ & & \\
Grade II $(n=10)$ & & \\
Grade IIla ( $n=3)$ & & \\
Grade IIIb ( $n=3)$ & & \\
Grade IV ( $n=1)$ & $26(13.3 \%)$ & $1(5.0 \%)$ \\
Lung* & $5(2.6 \%)$ & \\
Myocardium* (grade IIIa) ( $n=1$ patient) & $4(2.0 \%)$ & \\
Spleen & $3(1.5 \%)$ & \\
Bladder & & \\
Other: & $6(3.1 \%)$ & \\
m.psoas & $1(0.5 \%)$ & \\
m.adductores & $2(1.0 \%)$ & \\
Mesocolon & $2(1.0 \%)$ & \\
Retroperitoneum & $3(1.5 \%)$ & \\
Skin & 196 & \\
Total & $8.6(2-28)$ & \\
Diameter (cm), mean and range & & \\
\hline
\end{tabular}


Table 4. Primary and secondary outcomes of all 112 consecutive patients surgically treated for hydatid disease in the Harbour Hospital: 1981 - 2007.

\begin{tabular}{ll}
\hline Primary outcome & $9(8.0 \%)$ \\
Relapse of disease & $5(4.5 \%)$ \\
Intervention (re-operation) & \\
Secondary outcome & \\
Complications & $2(1.8 \%)$ \\
Grade I & $10(8.9 \%)$ \\
Grade II & $4(3.6 \%)$ \\
Grade IIIa & $3(2.7 \%)$ \\
Grade IIIb & $1(0.9 \%)$ \\
Grade IV(d) & 0 \\
Grade V (death) & $3(2.7 \%)$ \\
Re-operation & $14(2-156)$ days \\
Length of hospital stay (mean, range) & \\
\hline
\end{tabular}

Results are the number and percent of patients unless otherwise indicated

All but one of the complications were observed in the liver-operated group, which proved not to be of statistical significance $(P=0.477)$. Even after dividing the hydatid locations in only two groups -- liver (19/99 patients, or $19.2 \%$ complication rate) and other organs $(1 / 13$, or $7.7 \%$ complication rate) -- no significant difference was found ( $P=0.457)$. Also, no significant difference was seen when the grading of complications was analyzed $(P=0.881)$. However, if a complication occurred, these patients stayed significantly longer in hospital than the patients without complications $(P<0.001)$. The median stay was 20.5 days [10-156 days] in the group with complications $(n=20)$ versus 12 days [2-52 days] in those without complications $(n=87)$.

Of the 112 consecutive patients, 3 were treated with percutaneous technique prior to surgical treatment. This was decided in a multidisciplinary meeting prior to the intervention and it was considered suitable for treatment. The first patient needed a thoracoabdominal exploration because biliary pleuritis persisted after PAIR and required surgical treatment. Another patient was treated by PAIR for a hydatid cyst in the liver and was operated on with the frozen seal technique for the spleen without complication. The third patient was the previously described patient with ARDS (Grade IVd complication).

Spilling of cyst content was reported in four cases, without complications or recurrence in these patients during follow-up of 22.5 months (range 5-39 months). Two ERCPs were performed prior to the liver operation. An ERCP was done in the first patient to analyse the cause of the abnormal liver function tests. This patient was admitted in 1981 to the Department of Internal Medicine. After CT scanning he was referred to the Department of Surgery to be treated surgically. The second 
ERCP was performed 2 years later in a patient to analyze the cause of the persistant high bilirubin levels in the serum. The sonography showed an enlarged biliary tree and a cystic process in the right liver lobe. A parasitic infection was suspected at first, but serology was negative for echinococcosis. ERCP showed a stone in the common bile duct (CBD). Four days later, cholecystectomy with CBD exploration was planned. However, the "stone" seen on the ERCP proved to be a large daughter cyst and was removed. The large hydatid cyst in the liver was also treated, and T-tube drainage was performed. Postoperative medical treatment with mebendazole was started, and the patient was discharged in good condition 1 month later.

\section{DISCUSSION}

The present study showed that the use of a previously described frozen seal technique is safe and effective for the surgical treatment of hydatid disease in a nonendemic area. No mortality was seen in more than 25 years of surgically treated echinococcosis and the recurrence rate and morbidity were low. In fact, the results can be compared favourably to the recent meta-analysis where the clinical outcomes of 769 patients with hepatic echinococcosis treated with the PAIR technique were compared with 952 era-matched historical controls undergoing surgical intervention. ${ }^{8}$ In this meta-analysis of 14 studies on surgical treatment in endemic areas, minor and major complication rates of $33.0 \%$ and $25.1 \%$, respectively, were reported . The mortality rate was $0.7 \%(n=7)$ among the surgically treated patients. In a more recent single-centre study on this matter with 171 patients, a mortality rate of $1 \%$ was reported for 10 years of open surgical treatment. ${ }^{7}$ For this group of open surgery, the investigators observed a morbidity rate of $28 \%$ and recurrence rates of $16.2 \%$.

A drawback of the present study may be the retrospective setting, which could account for a bias for the comparison with reports in literature. However, most of the studies in the field of surgical treatment of hydatid disease are of the same retrospective design. ${ }^{2,7,24-31}$

There are different opinions concerning the optimal treatment for patients with hepatic echinococcal disease. The goal of treatment of hydatid disease is to eliminate the parasite completely and thereby prevent (or treat) complications including secondary infection, rupture of the cyst, anaphylactic reaction, pressure on adjacent structures and penetration into the biliary tract. It is also important to prevent recurrent disease, as it is known that there is a considerable rate of recurrence, possibly caused by intra-operative spilling. ${ }^{5,7,11,32}$

In theory, there are three treatment options for hepatic cystic echinococcosis: chemotherapy, surgery and percutaneous drainage or a combination of these therapies. ${ }^{7,8}$ However, the use of chemotherapeutic agents alone, such as mebendazole 
or albendazole, is controversial because of their limited efficacy. These antiparisitic drugs are often administered as adjuvant therapy during surgery or percutaneous treatment. In selected cases, they can be the primary approach when surgery is not feasible or is unsafe. . $^{8,10,32}$

Percutaneous drainage is another method for treating hepatic hydatid disease. The PAIR technique consists of several sequential steps: After percutaneous aspiration of the hydatid cyst under US or CT guidance, a protoscolicidal agent (20\% sodium chloride solution or $95 \%$ ethanol) is injected into the cyst cavity; the final step is reaspiration of the cyst contents. The advantage of PAIR is that it is less invasive. ${ }^{5,32}$ In the case of uncomplicated hepatic hydatid cysts, percutaneous drainage, in combination with albendazole therapy, is an effective, safe alternative to surgery and requires a shorter hospital stay. $2,3,7,10,31,33,34$ In this study, only three patients were treated with PAIR, with two complicated procedures, and these patients needed surgery thereafter.

The third method for managing cystic echinococcosis of the liver is surgery. The surgical approach remains the cornerstone of the treatment for hydatid disease. ${ }^{1,3,7,10,15,31,32,35}$ Some suggest that a combination of surgery and antiparasitic agents is the preferred treatment as it is associated with low morbidity, mortality and recurrences rates. ${ }^{10,36}$ Surgery of liver hydatid cysts is intended to remove the parasite completely by a procedure that includes safe, complete exposure of the cyst, safe decompression of the cyst, safe evacuation of the cyst contents, sterilization of the cyst, treatment of biliary complications, and management of the remaining cyst cavity. Surgeons must also try to prevent intraoperative spilling and to save healthy issue. $1,5,6,11,36$

The WHO has published a list of indications for surgical treatment: large liver cysts with multiple daughter cysts; single superficially situated liver cysts that may rupture spontaneously or as the result of a trauma; infected cysts; cysts communicating with the biliary tree and/or exerting pressure on adjacent vital organs and cysts in the lung, brain, kidney, bones and other organs. ${ }^{11,15}$

Important complications following surgery for hydatid liver echinococcosis are recurrences with a reported incidence of $1.1 \%$ to $25.0 \%$, which may be caused by spilling the cyst's contents during the previous operations. ${ }^{4,25,26,29,31,32,37}$ In the present study only four cases of intra-operative spilling were reported (3.6\%), without any recurrence in almost 2 years of median follow-up.

Surgical interventions can be divided into conservative, radical and laparoscopic procedures. Conservative, tissue-sparing techniques consist of evacuating the cyst and leaving the pericyst in situ, whereas radical procedures remove both the cyst and the pericyst. The most common conservative techniques include simple tube drainage, marsupialization, capitonnage, deroofing, partial cystectomy, and open or closed total cystectomy with or without omentoplasty. Conservative operations have good results regarding blood loss and length of hospital stay. ${ }^{36}$ 
Radical approaches consist of total pericystectomy or liver resection (hemihepatectomy or lobectomy) in which the cyst content and the entire pericystic membrane are removed. ${ }^{1,2,4-6,11}$ Radical resection means extensive dissection, leading to a large wound area with possibly increased risk of bleeding or bile leaks. ${ }^{2,33,36}$ This technique has also advantages for the treatment of hydatid disease. It is suggested to be a safe, highly effective technique with lower mortality, morbidity and recurrence rates than for conservative surgery. ${ }^{5,735}$ This can be partially explained by the fact that, compared to endocystectomy, excision of the cyst and pericyst minimizes the risk that daughter cysts persist in situ. ${ }^{10,36}$

Recurrences are also often related to spillage of the cyst content. ${ }^{7,33}$ Therefore, Saidi and Nazarian ${ }^{17}$ developed a method to prevent spillage of the cyst content . They devised a cone which simplifies the disinfection of the cyst cavity as used in this study. ${ }^{11,13,17,19}$ This procedure starts with mobilization of the liver so the cone can be centred on the apex of the cyst. After opening the cyst through the cone, the parasite is sucked off entirely, followed by disinfection of the remaining cavity. ${ }^{11,13,17,19}$

The laparoscopic approach has a growing role in the treatment of hepatic hydatid cysts. ${ }^{2,7,32,38}$ Current data suggest that this minimally invasive surgical technique is feasible, safe and effective. ${ }^{1,2,7,39-44}$ Unfortunately, randomized controlled trials are still lacking, which results in continuing debate about the optimal operation technique for hydatid disease. ${ }^{2,4,11,16,36}$ It is of major importance to select the appropriate therapy carefully, remembering the benign nature of this cystic disease. ${ }^{32}$ Careful patient selection is based on size, number and location of the cysts, the patient's characteristics, and the experience of the clinician. ${ }^{4,10}$

\section{Conclusions}

The frozen seal method of surgery from the past century is still safe and effective in the new millennium. This is especially useful in centres in nonendemic areas to facilitate working with a reproducible technique and, more importantly, to obtain high efficacy and low morbidity rates. Future studies are needed to prove its position in the treatment of hydatid disease as new developments show promising results.

Acknowledgements: We thank the surgical staff and Mrs M.E.I. van Nieuwland, Department of Surgery, Harbour Hospital for the cooperation and kind assistance with this study. We also thank Prof. Dr. C.H.C. Dejong for critically reading this manuscript. 


\section{REFERENCES}

1. Dervenis C, Delis S, Avgerinos C, et al. Changing concepts in the management of liver hydatid disease. J Gastrointest Surg. 2005; 9: 869-877.

2. Dziri $C$, Haouet $K$, Fingerhut A. Treatment of hydatid cyst of the liver: where is the evidence? World J Surg. 2004; 28: 731-736.

3. Khuroo MS, Wani NA, Javid G, et al. Percutaneous drainage compared with surgery for hepatic hydatid cysts. N Engl J Med. 1997; 337: 881-887.

4. Menezes dS. Hydatid cyst of the liver-criteria for the selection of appropriate treatment. Acta Trop. 2003; 85: 237-242.

5. Sayek I, Tirnaksiz MB, Dogan R. Cystic hydatid disease: current trends in diagnosis and management. Surg Today. 2004; 34: 987-996.

6. Smego RA, Jr., Sebanego P. Treatment options for hepatic cystic echinococcosis. Int J Infect Dis. 2005; 9: 69-76.

7. Yagci G, Ustunsoz B, Kaymakcioglu N, et al. Results of surgical, laparoscopic, and percutaneous treatment for hydatid disease of the liver: 10 years experience with 355 patients. World J Surg. 2005; 29: 1670-1679.

8. Smego RA, Jr., Bhatti S, Khaliq AA, et al. Percutaneous aspiration-injection-reaspiration drainage plus albendazole or mebendazole for hepatic cystic echinococcosis: a meta-analysis. Clin Infect Dis. 2003; 37: 1073-1083.

9. Ammann RW, Eckert J. Cestodes. Echinococcus. Gastroenterol Clin North Am. 1996; 25: 655-689.

10. Gourgiotis S, Stratopoulos C, Moustafellos $P$, et al. Surgical techniques and treatment for hepatic hydatid cysts. Surg Today. 2007; 37: 389-395.

11. Buttenschoen K, Carli BD. Echinococcus granulosus infection: the challenge of surgical treatment. Langenbecks Arch Surg. 2003; 388: 218-230.

12. Khuroo MS, Dar MY, Yattoo GN, et al. Percutaneous drainage versus albendazole therapy in hepatic hydatidosis: a prospective, randomized study. Gastroenterology. 1993; 104: 1452-1459.

13. Jongsma CK, Stuiver PC. [The surgical treatment of echinococcosis using a freezing method]. Ned Tijdschr Geneeskd. 1984; 128: 391-393.

14. Schipper HG, Kager PA. [Diagnosis and treatment of unilocular hydatid disease (Echinococcus granulosus infection)]. Ned Tijdschr Geneeskd. 1997; 141: 984-989.

15. Guidelines for treatment of cystic and alveolar echinococcosis in humans. WHO Informal Working Group on Echinococcosis. Bull World Health Organ. 1996; 74: 231-242.

16. Franciosi CM, Romano F, Porta G, et al. Surgical treatment of hydatid disease of the liver. An experience from outside the endemic area. Chir Ital. 2002; 54: 667-672.

17. Saidi F, Nazarian I. Surgical treatment of hydatid cysts by freezing of cyst wall and instillation of 0.5 per cent silver nitrate solution. N Engl J Med. 1971; 284: 1346-1350.

18. Regev A, Reddy KR, Berho M, et al. Large cystic lesions of the liver in adults: a 15-year experience in a tertiary centre. J Am Coll Surg. 2001; 193: 36-45.

19. Jongsma CK. The frozen seal technique in the surgical treatment of hydatid disease in the Netherlands. Acta Leiden. 1984; 52: 41-44.

20. Saidi F. A new approach to the surgical treatment of hydatid cyst. Ann R Coll Surg Engl. 1977; 59: 115-118.

21. Vrancken Peeters MP, Vrancken Peeters MJ, Corion LU, et al. Quality control of colorectal surgery with an extensive complication registration system. Dig Surg. 2005; 22: 168-173.

22. Clavien PA, Sanabria JR, Strasberg SM. Proposed classification of complications of surgery with examples of utility in cholecystectomy. Surgery. 1992; 111: 518-526.

23. Sielaff TD, Taylor B, Langer B. Recurrence of hydatid disease. World J Surg. 2001; 25: 83-86.

24. Akcan A, Akyildiz H, Artis T, et al. Peritoneal perforation of liver hydatid cysts: clinical presentation, predisposing factors, and surgical outcome. World J Surg. 2007; 31: 1284-1291. 


\section{Chapter 3}

25. Alfieri S, Doglietto GB, Pacelli F, et al. Radical surgery for liver hydatid disease: a study of 89 consecutive patients. Hepatogastroenterology. 1997; 44: 496-500.

26. Balik AA, Basoglu M, Celebi F, et al. Surgical treatment of hydatid disease of the liver: review of 304 cases. Arch Surg. 1999; 134: 166-169.

27. Chautems R, Buhler LH, Gold B, et al. Surgical management and long-term outcome of complicated liver hydatid cysts caused by Echinococcus granulosus. Surgery. 2005; 137: 312-316.

28. Karavias DD, Vagianos CE, Kakkos SK, et al. Peritoneal echinococcosis. World J Surg. 1996; 20: 337340.

29. Magistrelli P, Masetti R, Coppola R, et al. Surgical treatment of hydatid disease of the liver. A 20year experience. Arch Surg. 1991; 126: 518-522.

30. Sayek I, Yalin R, Sanac Y. Surgical treatment of hydatid disease of the liver. Arch Surg. 1980; 115: 847-850.

31. Yorganci K, Sayek I. Surgical treatment of hydatid cysts of the liver in the era of percutaneous treatment. Am J Surg. 2002; 184: 63-69.

32. Voros D, Katsarelias D, Polymeneas G, et al. Treatment of hydatid liver disease. Surg Infect (Larchmt). 2007; 8: 621-627.

33. Kayaalp C, Sengul N, Akoglu M. Importance of cyst content in hydatid liver surgery. Arch Surg. 2002; 137: 159-163.

34. Ustunsoz B, Akhan O, Kamiloglu MA, et al. Percutaneous treatment of hydatid cysts of the liver: long-term results. AJR Am J Roentgenol. 1999; 172: 91-96.

35. Alonso CO, Moreno GE, Loinaz SC, et al. Results of 22 years of experience in radical surgical treatment of hepatic hydatid cysts. Hepatogastroenterology. 2001; 48: 235-243.

36. Mueller L, Broering DC, Vashist $Y$, et al. A retrospective study comparing the different surgical procedures for the treatment of hydatid disease of the liver. Dig Surg. 2003; 20: 279-284.

37. Vagianos CE, Karavias DD, Kakkos SK, et al. Conservative surgery in the treatment of hepatic hydatidosis. Eur J Surg. 1995; 161: 415-420.

38. Cirenei A, Bertoldi I. Evolution of surgery for liver hydatidosis from 1950 to today: analysis of a personal experience. World J Surg. 2001; 25: 87-92.

39. Altinli E, Saribeyoglu K, Pekmezci S, et al. An effective omentoplasty technique in laparoscopic surgery for hydatid disease of the liver. JSLS. 2002; 6: 323-326.

40. Bickel A, Loberant N, Singer-Jordan J, et al. The laparoscopic approach to abdominal hydatid cysts: a prospective nonselective study using the isolated hypobaric technique. Arch Surg. 2001; 136: 789-795.

41. Chowbey PK, Shah S, Khullar R, et al. Minimal access surgery for hydatid cyst disease: laparoscopic, thoracoscopic, and retroperitoneoscopic approach. J Laparoendosc Adv Surg Tech A. 2003; 13: 159165.

42. Ertem M, Karahasanoglu T, Yavuz N, et al. Laparoscopically treated liver hydatid cysts. Arch Surg. 2002; 137: 1170-1173.

43. Khoury G, Abiad F, Geagea T, et al. Laparoscopic treatment of hydatid cysts of the liver and spleen. Surg Endosc. 2000; 14: 243-245.

44. Manterola C, Fernandez O, Munoz S, et al. Laparoscopic pericystectomy for liver hydatid cysts. Surg Endosc. 2002; 16: 521-524. 


\title{
Chapter 4
}

\section{Hepatic echinococcosis in a non-endemic region:}

\author{
Trends in incidence, diagnosis and treatment
}

J.H.M.B. Stoot, R.J.S. Coelen, T. Herremans, M. Casparie, J.J. Verweij, H. Schipper

L. Kortbeek and C.H.C. Dejong

Contributors: I. Limantoro, A. de Bruïne

In collaboration with the Dutch Liver Surgery Collaborative Group

Submitted

Presented to the 9th World Congress of the International Hepato-Pancreato-Biliary Association in Buenos Aires, Argentina, April 2010. Part of the data was previously presented in Dutch in the online Ned Tijdschr Geneesk. 2010; 154:A2297 


\section{ABSTRACT}

Background: The aim of this study was to investigate (1) the incidence of hepatic echinococcosis in a non-endemic country and to explore the opinions of experts concerning (2) diagnosis and (3) treatment of echinococcosis.

Methods: Data were extracted in three ways. The national database of pathology (PALGA), containing all individual pathology reports, was investigated and compared with results from serological testing from 1997 till 2008. In addition, a questionnaire concerning incidence, diagnosis, therapy and follow-up, was sent to gastrointestinal surgeons in 20 Dutch hepato-pancreato-biliary centres.

Results: A decrease was observed in the number of resected specimens (PALGA) from 2003 onwards. The incidence of echinococcosis based on serology decreased from 2002 onwards to 33 patients per year. 17/20 hospitals responded to the questionnaire. 7/20 centres stated they had treated hydatid disease of the liver. The diagnosis was in most cases based on clinical criteria, computed tomography and serology. The primary treatment strategy varied considerably between hospitals.

Conclusion: The incidence of hepatic hydatid disease in the Netherlands is decreasing in the past decade. Since this remains a rare disease in non-endemic areas and the primary treatment strategy differs between specialised centres, a 'step-up' treatment protocol might help improve uniformity in treatment strategies. 


\section{INTRODUCTION}

Human cystic echinococcosisis is one of the most neglected parasitic diseases in the world. In many endemic regions, infected patients suffer considerably from this disease, usually because of the lack of treatment possibilities due to poor infrastructure and shortage of drugs and equipment. ${ }^{1,2}$ This parasitic infection, also named hydatid disease, is caused by the larval forms of the dog tapeworm Echinococcus granulosus. ${ }^{3-5}$ Worldwide, echinococcosis remains endemic in large sheepraising areas. These areas include the Mediterranean and Eastern parts of Europe, Africa, the Middle East, Asia, South America, Australia and New Zealand. The incidence in Western countries is relatively low. ${ }^{3,6-8}$ However, immigration and travelling has led to an increase of the prevalence of this disease in Northern parts of Europe and North America., ${ }^{9,10}$ Echinococcosis in native Dutch inhabitants occurs rarely since the introduction of extensive screening of livestock after slaughter. Most of the patients identified with echinococcosis are immigrants from countries in the Mediterranean area, where the disease is endemic, especially in Turkey and Morocco. ${ }^{11}$ From 1987-1991, each year 29 to 44 new patients were diagnosed in the Netherlands with echinococcosis based on serology. ${ }^{11}$ The diagnosis of hepatic echinococcosis can be made with a combination of clinical history, liver imaging and serology and parasitological examination of cyst fluid. For the serodiagnosis of echinococcosis infection 'enzyme-linked immunosorbent assay' (ELISA) and 'indirect hemagglutination assay' (IHA) can be used as a screening tool, while immunoelectrophoresis or immunoblotting confirm the diagnosis echinococcosis. ${ }^{5,11} \mathrm{Mi}$ croscopy of the cyst fluid or pathologic examination of the cyst content provide additional confirmation of the diagnosis.

Treatment of hepatic cystic echinococcosis can be either by medical therapy, by surgery or by percutaneous drainage (Puncture Aspiration Injection and Reaspiration, also known as PAIR) or if necessary a combination of these therapies. ${ }^{3,7}$ Until now, there is no consensus about the best treatment. The international WHO IWGE guideline states that surgery is the cornerstone of treatment and percutaneous techniques may constitute an alternative. However, in the era of minimal invasive techniques the question is whether clinicians still follow this guideline. Looking at the surgical approach to hepatic hydatid disease the clinician can choose between liver sparing or 'conservative' treatment (cystectomy), radical treatment (complete open resection) and minimally invasive or laparoscopic techniques. There is still discussion about the best surgical method; whether this is liver sparing or radical surgery, in which the cyst is totally removed including the pericyst by total pericystectomy or partial hepatectomy. ${ }^{12}$ In areas where hydatid disease is non-endemic and consequently surgical treatment is no routine procedure, it is important to perform safe and reproducible operations with a low complication rate. The frozen seal method as a surgical treatment for hydatid disease was re- 
cently reported to be still safe and effective. ${ }^{13}$ However, these authors ${ }^{13}$ observed a gradual decrease in number of surgical hepatic operations. The question arose whether this was due to the change in treatment to less invasive techniques or to a general decrease in incidence of echinococcosis. As in many countries in the world, human cases of echinococcosis is not a notifiable disease in the Netherlands. Consequently, there are no reliable numbers about the incidence and prevalence. ${ }^{11}$ The aim of this study was to investigate the incidence of hepatic echinococcosis in the context of the total echinococcosis incidence in a non-endemic country in Europe and also, to obtain information on diagnosis and current treatments in the surgical community.

\section{METHODS}

Data on the incidence of hepatic echinococcosis in the Netherlands were extracted in three ways. First, the national database of pathology (PALGA), the nationwide network and registry of histo- and cytopathology in the Netherlands, was investigated from 1997 to 2008 . This database incorporates all individual reports of biopsies and resected specimens in the Netherlands and is a complete resource for all pathology specimens in the Netherlands since $1991 .{ }^{14}$ Patient information from this database was provided anonymously. However, data on sex, date of birth and date of specimen retrieval (pathology report) were given after approval of the medical ethics committee.

In addition to this, serology data of patients in the Netherlands were examined by consulting the database of The National Institute for Public Health and the Environment (RIVM), together with the databases of Leiden University Medical Centre (LUMC) and the Academic Medical Centre Amsterdam (AMC) in the study period. A serologic ELISA test was used to screen all retrieved cases. ${ }^{15}$ After a positive result of the IgG ELISA for echinococcosis infection, confirmation tests were done. In the earlier days of the time frame studied, Immuno Electrophoresis (IEP) or Complement Binding Reactions (CBR) were used, whereas more recently IgG1 and IgG4 immunoblot (RIVM) or IgG1, IgG4 and IgE ELISA (LUMC) were used as confirmation tests. Polymerase Chain Reaction (PCR) was performed when cyst content was available. ${ }^{16}$ All patients that had tested positive for serology, were included in this study. Moreover, the yearly numbers of immigrants were obtained from the Central Bureau for Statistics (CBS) in the Netherlands (www.statline.cbs.nl).

Finally, a questionnaire was sent to gastrointestinal surgeons in 20 hepatopancreato-biliary (HPB) centres in hospitals in the Netherlands to get an overview of their opinions on hepatic echinococcosis. The questionnaire included mostly multiple-choice questions about the incidence, diagnosis, therapy and follow-up of 
liver echinococcosis (appendix 1). The questionnaires were sent by email and reminders were sent afterwards by email or personal contact.

\section{Statistics}

Continuous variables are presented as mean +/- SD or median and range, when appropriate. Categorical variables are presented as absolute amount (percentages). Spearman's correlation coefficients were calculated between immigrants and number of patients diagnosed with hydatid disease based on laboratory testing. A p-value $<0.05$ was considered statistically significant.

\section{RESULTS}

\section{Incidence of hepatic echinococcosis based on pathology database}

Histologic examinations were performed in a mean of 8 (2-14) newly diagnosed patients per year. The data were divided into three groups; liver resection specimens, puncture or biopsy specimens and specimens from other organs/sites. A decrease was seen in the number of resected liver specimens from 2003 onwards (figure 1). Since the PALGA data were provided anonymously, no in depth investigation for overlap of newly diagnosed patients between the PALGA and serology database could be made.

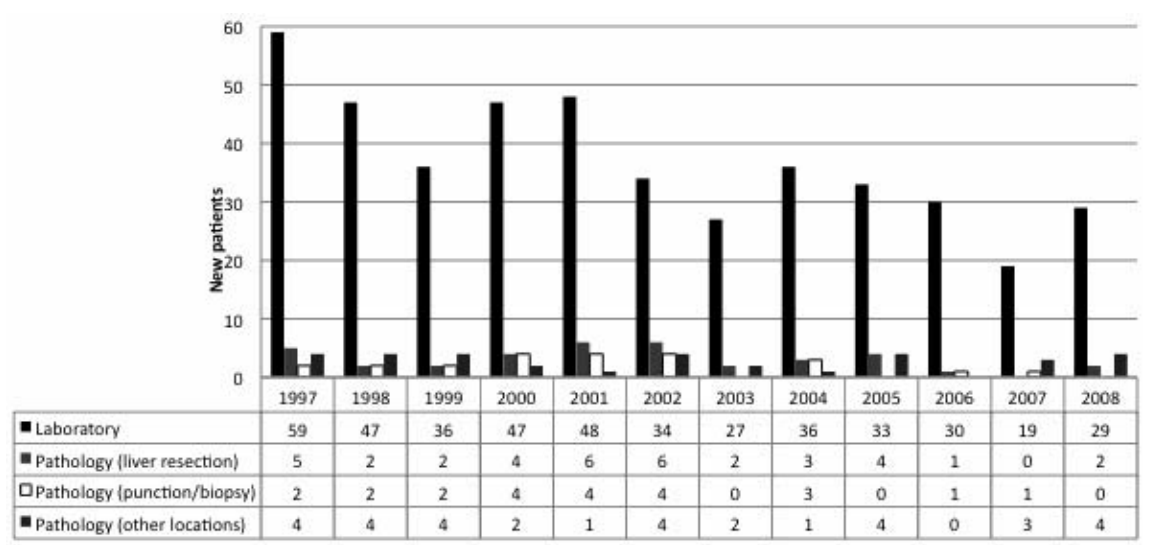

Figure 1. Trends in incidence of echinococcosis in the Netherlands, based on nationwide serology laboratory data and histopathology data. 


\section{Incidence of echinococcosis based on serology databases}

All patients in the Netherlands suspected of hydatid disease were investigated by serology testing. A total of 7314 sera from 5125 patients were investigated for antibodies from 1997 to 2008 . Between 550 to approximately 600 new tests were performed every year. In the study period, 485 patients tested positive on the screening test (IgG ELISA). In 445 of these 485 patients, the confirmation tests were also positive (92\%).

Between 1997 and 2001 the mean incidence was 51 (36-59) patients per year, while from 2002 onwards, a decrease in incidence to 33 (19-36) patients per year was noticed. The number of immigrants in the Netherlands decreased in the same period and followed the same trend as the incidence of new patients with hydatid disease (Figure 2). The number of immigrants failed to attain a significant correlation with the number of patients tested positive for hydatid disease $(p=0.561$, $\mathrm{R}=0.187)$.

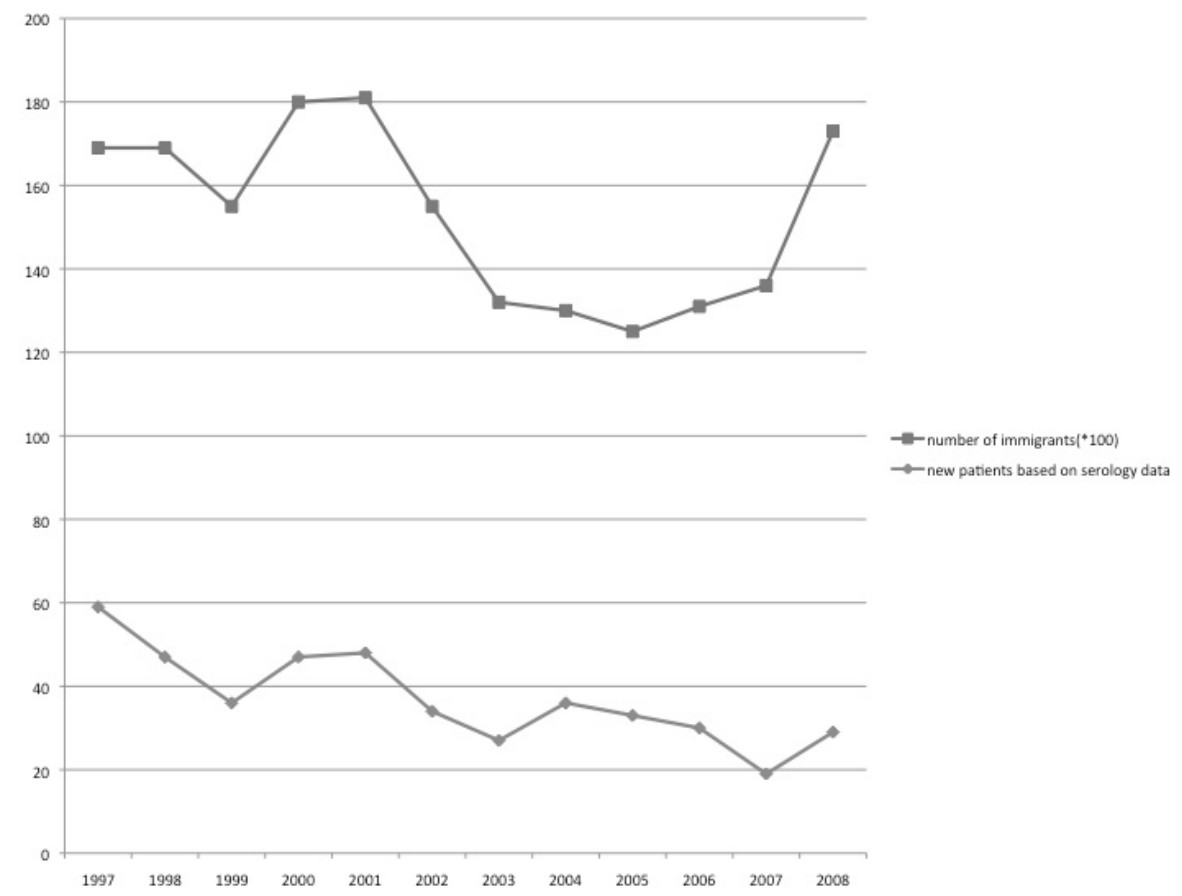

Figure 2. Trends in number of immigrants admitted to the Netherlands and the incidence of echinococcosis based on serology data. Number of immigrants are converted (www.statline.cbs.nl). 


\section{Outcome of the questionnaire}

Seventeen (85\%) of twenty hospitals receiving the questionnaire responded (seven of eight University Hospitals and ten of twelve District General hospitals). Only seven (41\%) centres stated they had treated hydatid disease of the liver. An estimation of the incidence was provided by three of these seven hospitals whereas the other four provided rates based on actual data. Actual data of the two largest centres are presented in table 1.

Table 1. Trends in incidence, diagnosis and treatment of the two largest treatment centres based on actual data from 1990 onwards.

\begin{tabular}{|c|c|c|}
\hline Centres & AMC & Harbour Hospital \\
\hline \multicolumn{3}{|l|}{ Incidence } \\
\hline No. of patients with hepatic hydatidosis & 131 & 48 \\
\hline solitairy hydatid cyst of the liver & n.a. & 34 \\
\hline Multiple cysts of the liver & n.a. & 14 \\
\hline \multicolumn{3}{|l|}{ Diagnosis } \\
\hline radiology(CT/MRI/sonography) and serology & all & all \\
\hline confirmation (ELISA/IHA) & all & all \\
\hline \multicolumn{3}{|l|}{ Treatment } \\
\hline medication & yes & yes \\
\hline Surgery & not primarily & yes \\
\hline - cystectomy & yes & no \\
\hline - pericystectomy & no & no \\
\hline - hemihepatectomy & yes & no \\
\hline - frozen seal method & no & yes \\
\hline PAIR $^{a}$ & yes & rarely \\
\hline PEVAC $^{b}$ & yes & no \\
\hline \multicolumn{3}{|l|}{ Interventions } \\
\hline surgery & 12 & 48 \\
\hline PAIR $^{a}$ & 24 & 1 \\
\hline PEVAC $^{b}$ & 51 & - \\
\hline \multicolumn{3}{|l|}{ Recurrences } \\
\hline surgery & 0 & 5 \\
\hline PAIR $^{a}$ & 5 & 1 \\
\hline PEVAC $^{b}$ & 4 & - \\
\hline
\end{tabular}

${ }^{a}$ PAIR: Puncture Aspiration Injection and Reaspiration

${ }^{b}$ PEVAC: percutaneous evacuation of cyst content

n.a: not available

AMC: Academical Medical Centre, Amsterdam. Harbour Hospital: Centre for Tropical Diseases, Rotterdam

Six (86\%) of seven centres treated one to five patients per year and two of these centres had treated only one patient since 1990. The estimated total number of 
patients treated for hepatic echinococcosis since 1990 was more than 200 and varied from 1 to 131 patients per centre.

Diagnosis. The preoperative diagnosis of hepatic hydatid disease was in all patients made using computed tomography (CT) and serology analysis. In 3 centres, ultrasonography was also used, and in another 3 centres, the diagnosis was also based on clinical criteria. No changes in diagnostic procedures were observed in the study period. Usually, ELISA was used as a screening method and IEP or immunoblotting to confirmation test. Finally, microscopy and parasitology was used to confirm the presumed diagnosis.

Treatment. In four of the seven hospitals (57\%) treatment was protocol based. The treatment strategy was in all centres discussed in a multidisciplinary team, which consisted in five centres of a surgeon, internist and radiologist. Primary treatment strategy varied between the hospitals. Medication (as main treatment) was administered in five (71\%) of seven hospitals. PAIR was used as primary treatment strategy in only two (29\%) of seven centres. In five (71\%) of seven centres, surgery was the first choice treatment in case of severe complaints or when percutaneous treatment could not safely or effectively be performed. In the largest centre (AMC), percutaneous treatment of hydatid cysts was introduced stepwise. PAIR was started in 1996 as the first choice of treatment of unilocular hydatid cysts. Since 1998, the percutaneous evacuation of cyst content (PEVAC) was the preferred treatment of multilocular ("mother and daughter") cysts in this centre. ${ }^{17}$ To reduce procedure related infections, PEVAC was modified extensively in 2007.

Follow-up. Follow-up after surgery varied between the centres. Diagnostics used during follow-up were CT in five (71\%) hospitals, ultrasonography in 2 (29\%), MRI in $1(14 \%)$ and serology in $2(29 \%)$ centres.

\section{DISCUSSION}

This study reports the incidence, diagnosis and treatment strategies of hepatic hydatid disease in a non-endemic country. Data were acquired by consulting the national database of pathology, the databases of the laboratories where serology was tested and by conducting a survey amongst surgeons in HPB centres. To our knowledge, this nationwide multidisciplinary approach is the first such study in a non-endemic country in northern Europe. These data show a decreasing incidence of hepatic hydatidosis in the past decade. After the introduction of percutaneous treatment, a tendency was observed to adopt this less invasive technique as first choice treatment above surgery in the largest centre. 


\section{Trends in Incidence}

Since human echinococcosis is not an a notifiable disease in the majority of countries worldwide, it remains a challenge to estimate its true incidence. ${ }^{18}$ In highly endemic areas, such as parts of China, an incidence of 42/100.000 has been reported. ${ }^{19}$ In some endemic European areas, the incidence is much lower and varies between less than 1 and > 8/100.000. Little is known about the incidence in nonendemic European countries. In the last decade, we observed a decreasing incidence of hepatic hydatidosis in The Netherlands from 51(36-59) to 33(19-36) patients per year. Similar observations were reported from endemic areas in Europe. ${ }^{20-22}$ This contrasts to the highly endemic parts elsewhere in the world. ${ }^{23-25}$ The decreasing incidence of hepatic hydatidosis in the Netherlands might partially be ascribed to the strict policy of the Dutch Government. Since the introduction of the Dutch law on foreigners in 2000, the number of immigrants decreased steadily from 133,404 in 2001 to 92,297 immigrants in 2005 (http://nl.wikipedia.org/wiki/Immigratiebeleid_(Nederland)\#Asielzoekers_2). The trend of decreasing incidence of hepatic hydatidosis seems to follow the trend of immigrants in the Netherlands (figure 2).

\section{Trends in diagnosis}

No changes in diagnostic procedures were observed in the study period.

\section{Trends in treatment}

In the recent revision of the guideline by the WHO IWGE ${ }^{26}$ and other reports ${ }^{8,27}$ it was stated that surgery remains the cornerstone of treatment of human echinococcossis, as it has the potential to remove the hydatid cyst and lead to complete cure. It is advised to evaluate surgery carefully against other less invasive options like percutaneous interventions. Percutaneous treatment such as PAIR, was considered to be an alternative for surgery and indicated for inoperable patients or patients who refuse surgery. ${ }^{28}$ Chemotherapy is indicated for inoperable patients with hydatid liver disease or multiple cysts in two or more organs. It is advised as a treatment to prevent secondary echinococcosis and recommended preoperatively and peri-interventionally for PAIR. ${ }^{26}$

\section{Surgery}

Regarding surgical treatment in the study, only a minority of newly diagnosed patients with hydatid disease were treated with surgery. The actual percentage of surgical cases in relation to the incidence remained stable over the years. Although we found considerable differences in treatment strategies between centres (table 
1), the outcome of the survey shows a trend towards less invasive treatment. This is consistent with the recent WHO guideline, and with more recent publications on this issue where minimally invasive and percutaneous treatments are reported to be safe and effective. ${ }^{3,4,29}$ Upon reviewing the literature, it seems that in recent years a gradual shift from open surgery towards minimally invasive and percutaneous treatment strategies has taken place. A relatively new minimally invasive technique for hydatid cysts in the liver is the laparoscopic approach. This technique can be radical and incorporates the benefits of the laparoscopic approach such as less pain, faster recovery and shorter hospital length of stay, compared to open surgery. $^{30}$

\section{Percutaneous therapy}

This survey among Dutch HPB centres demonstrates that percutaneous treatment of hydatid cysts such as PAIR and PEVAC is mainly used in one single specialised centre and rarely in the other centres. In the largest centre, PAIR is preferred as first choice treatment in patients with unilocular cysts and PEVAC in patients with multilocular cysts. ${ }^{17}$ When these techniques cannot be applied safely or effectively, surgery is indicated. This preference for percutaneous treatment is supported by the meta-analysis by Smego et al. PAIR was shown to be superior in terms of clinical and parasitological efficacy, morbidity, mortality, disease recurrence and hospital stay. ${ }^{3}$ Others have also reported that the percutaneous technique should be considered first in selected patients but only when the expertise is available. ${ }^{31}$

\section{Medical treatment}

Medical therapy consists of benzimidazole carbamates (mebendazole and albendazole). These anthelmintic drugs destroy the parasite by impairing glucose metabolism. ${ }^{31}$ Albendazole is superior to mebendazole due to its better absorption. ${ }^{31}$ In the majority of Dutch treatment centres, albendazole is preventively started before invasive treatment is initiated. Although necessary as adjunct to invasive treatments and successful for prevention of complications, the efficacy of chemotherapy alone as treatment was reported to be overestimated in the past. Fourty percent of the cysts did not respond to medical treatment or relapsed after treatment. $^{32}$

The present survey revealed striking differences in the treatment of hepatic hydatosis between the centres. To improve outcome, consensus on treatment is needed. Therefore, a 'step-up' algorithm for a treatment strategy for non-endemic Western countries is suggested (Figure 3 ), based on this study and recent evidence reported in literature. $3,4,8,29,31-34$ 


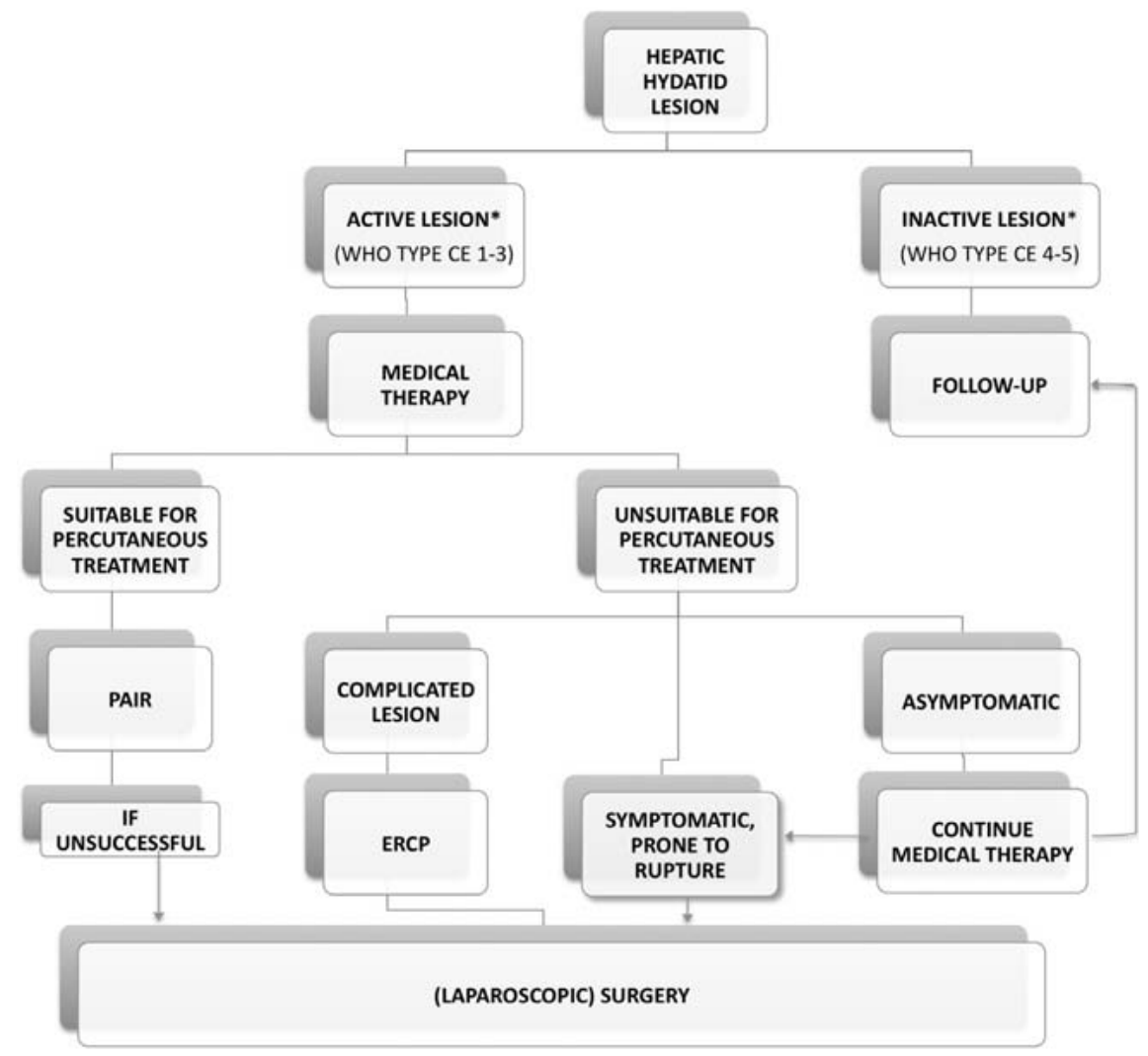

Figure 3. A 'step-up' algorithm for treatment of hydatid lesions based on the recent evidence and results of this current survey. $3,4,8,29,31-34$

The World Health Organization Informal Working Group on Echinococcosis (WHO-IWGE) classification of hydatid liver lesions is based on the Gharbi classification. Five morphologic types in three clinical stages have been described previously: types CE 1 and 2 (cystic echinococcosis) indicate active lesions; type CE 3 indicates transitional lesions which usually contain viable protoscolices; types CE 4 and 5 indicate inactive lesions. The lesions are further classified as small $(<5 \mathrm{~cm})$, medium $(5-10 \mathrm{~cm})$ or large $(>10 \mathrm{~cm})$.

\section{Conclusions}

The combined databases of pathology and serology indicate that the incidence of hydatid disease of the liver in the Netherlands remains rare. The current survey demonstrated that new guidelines are needed to agree on uniform primary treatment strategies. In the Netherlands, only two large centres are focussing on treatment of patients with hydatidosis. Centralization of this rare disease might improve the results as proven in other surgical treatments. Only then, can centres increase their expertise and a gradual shift to less invasive and safer treatment is to be expected. Management of this disease should be multidisciplinary and custom made, 
and a 'step-up' treatment protocol is provided, which may help to standardize treatment.

Acknowledgements: We thank the surgical staff, Dr Jongsma, and Mrs M.E.I. van Nieuwland, secretary of Department of Surgery of the Harbour Hospital in Rotterdam, the Netherlands, for the cooperation and kind assistance for this study. We also would like to thank dr J.W van den Esschert of the Department of Surgery from the Academic Medical Centre in Amsterdam, The Netherlands, for her work and contribution to the survey. Furthermore, we would like to thank our collegues from the Dutch Liver Surgery Group for the participation in the survey. Special thanks to the collaborators: M.A. Cuesta (Free University Medical Centre, Amsterdam), O.T. Terpstra (Leiden University Medical Centre, Leiden), O.R. Busch (Academical Medical Centre, Amsterdam), M.T. De Boer (University Medical Centre Groningen, Groningen), P. Van Duijvendijk (Radboud hospital, Nijmegen), G. Kazemier, C. Verhoef (Erasmus MC, Rotterdam), R. Van Hillegersberg (University Medical Centre Utrecht, Utrecht), A.M. Rijken (Amphia Hospital, Breda), M.S.L. Liem (Deventer Hospital, Deventer), R. Roumen (Medical Maxima Centre, Veldhoven), J. Klaasse (Medical Spectrum Twente, Enschede), J.Pierie (Leeuwarden Medical Centre, Leeuwarden), K. Hulsewe (Orbis Medical Centre, Sittard), G.A. Patijn (Isala klinieken, Zwolle).

\section{REFERENCES}

1. Seimenis A. Overview of the epidemiological situation on echinococcosis in the Mediterranean region. Acta Trop 2003; 85: 191-195.

2. Menezes da Silva AM. Human echinococcosis: a neglected disease. Gastroenterol Res Pract 2010; pii: 583297.

3. Smego RA, Jr., Bhatti S, Khaliq AA, et al. Percutaneous aspiration-injection-reaspiration drainage plus albendazole or mebendazole for hepatic cystic echinococcosis: a meta-analysis. Clin Infect Dis 2003; 37: 1073-1083.

4. Dziri C, Haouet K, Fingerhut A. Treatment of hydatid cyst of the liver: where is the evidence? World J Surg 2004; 28: 731-736.

5. Dervenis C, Delis S, Avgerinos C, et al. Changing concepts in the management of liver hydatid disease. J Gastrointest Surg 2005; 9: 869-877.

6. Gourgiotis S, Stratopoulos C, Moustafellos $P$, et al. Surgical techniques and treatment for hepatic hydatid cysts. Surg Today 2007; 37: 389-395.

7. Smego RA, Jr., Sebanego P. Treatment options for hepatic cystic echinococcosis. Int J Infect Dis 2005; 9: 69-76.

8. Buttenschoen K, Carli Buttenschoen D. Echinococcus granulosus infection: the challenge of surgical treatment. Langenbecks Arch Surg 2003; 388: 218-230.

9. Khuroo MS, Dar MY, Yattoo GN, et al. Percutaneous drainage versus albendazole therapy in hepatic hydatidosis: a prospective, randomized study. Gastroenterology 1993; 104: 1452-1459.

10. Sayek I, Tirnaksiz MB, Dogan R. Cystic hydatid disease: current trends in diagnosis and management. Surg Today 2004; 34: 987-996. 
11. Kortbeek LM, van Knapen F, Polderman AM, et al. [Echinococcosis in The Netherlands, 1987-1991]. Ned Tijdschr Geneeskd 1993; 137: 2715-2716.

12. Franciosi CM, Romano F, Porta G, et al. Surgical treatment of hydatid disease of the liver. An experience from outside the endemic area. Chir Ital 2002; 54: 667-672.

13. Stoot $\mathrm{JH}$, Jongsma CK, Limantoro I, et al. More than 25 years of surgical treatment of hydatid cysts in a nonendemic area using the 'frozen seal' method. World J Surg 2010; 34: 106-113.

14. Casparie M, Tiebosch AT, Burger G, et al. Pathology databanking and biobanking in The Netherlands, a central role for PALGA, the nationwide histopathology and cytopathology data network and archive. Cell Oncol 2007; 29: 19-24.

15. Force L, Torres JM, Carrillo A, et al. Evaluation of eight serological tests in the diagnosis of human echinococcosis and follow-up. Clin Infect Dis 1992; 15: 473-480.

16. Gasser RB, Zhu X, McManus DP. Display of sequence variation in PCR-amplified mitochondrial DNA regions of Echinococcus by single-strand conformation polymorphism. Acta Trop 1998; 71: 107-115.

17. Schipper HG, Lameris JS, van Delden OM, et al. Percutaneous evacuation (PEVAC) of multivesicular echinococcal cysts with or without cystobiliary fistulas which contain non-drainable material: first results of a modified PAIR method. Gut 2002; 50: 718-723.

18. Dakkak A. Echinococcosis/hydatidosis: a severe threat in Mediterranean countries. Vet Parasitol 2010; 174: 2-11.

19. Eckert J, Deplazes P. Biological, epidemiological, and clinical aspects of echinococcosis, a zoonosis of increasing concern. Clin Microbiol Rev 2004; 17: 107-135.

20. Gabriele F, Bortoletti G, Conchedda M, et al. [Human cystic hydatidosis in Italy: a public health emergency? Past to present]. Parassitologia 2004; 46: 39-43.

21. Benner C, Carabin H, Sanchez-Serrano LP, et al. Analysis of the economic impact of cystic echinococcosis in Spain. Bull World Health Organ 88: 49-57.

22. Sotiraki S, Himonas C, Korkoliakou P. Hydatidosis-echinococcosis in Greece. Acta Trop 2003; 85: 197-201.

23. Gavidia CM, Gonzalez AE, Zhang W, et al. Diagnosis of cystic echinococcosis, central Peruvian Highlands. Emerg Infect Dis 2008; 14: 260-266.

24. Torgerson PR, Karaeva RR, Corkeri N, et al. Human cystic echinococcosis in Kyrgystan: an epidemiological study. Acta Trop 2003; 85: 51-61.

25. Torgerson PR, Shaikenov BS, Baitursinov KK, et al. The emerging epidemic of echinococcosis in Kazakhstan. Trans R Soc Trop Med Hyg 2002; 96: 124-128.

26. Brunetti E, Kern P, Vuitton DA. Expert consensus for the diagnosis and treatment of cystic and alveolar echinococcosis in humans. Acta Trop 2010; 114: 1-16.

27. Filippou D, Tselepis D, Filippou G, et al. Advances in liver echinococcosis: diagnosis and treatment. Clin Gastroenterol Hepatol 2007; 5: 152-159.

28. Guidelines for treatment of cystic and alveolar echinococcosis in humans. WHO Informal Working Group on Echinococcosis. Bull World Health Organ 1996; 74: 231-242.

29. Yagci G, Ustunsoz B, Kaymakcioglu N, et al. Results of surgical, laparoscopic, and percutaneous treatment for hydatid disease of the liver: 10 years experience with 355 patients. World J Surg 2005; 29: 1670-1679.

30. Manterola C, Fernandez O, Munoz S, et al. Laparoscopic pericystectomy for liver hydatid cysts. Surg Endosc 2002; 16: 521-524.

31. Schipper HG, Kager PA. Diagnosis and treatment of hepatic echinococcosis: an overview. Scand J Gastroenterol Suppl 2004; 241: 50-55.

32. Stojkovic M, Zwahlen M, Teggi A, et al. Treatment response of cystic echinococcosis to benzimidazoles: a systematic review. PLoS Negl Trop Dis 2009; 3: e524.

33. Dziri C, Haouet K, Fingerhut A, et al. Management of cystic echinococcosis complications and dissemination: where is the evidence? World J Surg 2009; 33: 1266-1273.

34. Rozanes I, Guven K, Acunas B, et al. Cystic echinococcal liver disease: new insights into an old disease and an algorithm for therapy planning. Cardiovasc Intervent Radiol 2007; 30: 1112-1116. 
Chapter 4

\section{APPENDIX 1. QUESTIONNAIRE}

Incidence

1. How many patients with hydatid disease of the liver are treated in your hospital each year?

a 0

口 1-5

6-10

$\square>10$

2. How were patients referred to your hospital?

no referral

$\square$ first line (general practitioner, medical immigration service)

$\square$ second line (other hospitals)

$\square$ other, please specify: ...

\section{Diagnosis}

3. Which method is used to diagnose echinococcosis of the liver.

$\square$ clinically

$\square$ ultrasonography

CT scan

$\square \mathrm{MRI}$

serology

$\square$ other, please specify: ...

4. How is the diagnosis confirmed?

no confirmation

ELISA (enzyme-linked immunosorbent assay)

I IHA (indirect hemagglutination)

\section{Treatment}

5. Is there a protocol for the treatment of hydatid cysts?

no

$\square$ yes

6. Is there a multidisciplinary meeting before treatment

$\square$ yes

a no

With which specialities:

$\square$ surgeons

internists/gastroenterologists

口 (interventional) radiologists

7. What is the indication for treatment

$\square$ abdominal pain

$\square$ jaundice

$\square$ dyspnea 
$\square$ anorexia

$\square$ other, please specify: . . .

8. What is the primary treatment strategy in your hospital?

$\square$ medication

PAIR(Percutaneous Aspiration Injection Reaspiration)

$\square$ surgery

$\square$ only surgery by severe complaints

$\square$ other, please specify: ...

9. If applicable, what medication and what dosage is used?

$\square$ albendazol dosage:

$\square$ mebendazol dosage:

$\square$ praziquentel dosage:

$\square$ other, please specify: ...

10. If surgery is primary treatment, is medication administered preoperatively?

$\square$ no

$\square$ yes if applicable, when $\square 1$ day

$\square 1$ week

$\square$ other, please specify: ...

11. If surgery is primary treatment, what type of surgery is preferred

$\square$ (subtotal) cystectomy

$\square$ pericystectomy

$\square$ hemi-hepatectomy

$\square$ frozen seal method

$\square$ other, please specify: . . .

12. Approximately how many patients with hydatid liver cysts treated with PAIR since 1990 ?

13. Approximately how many patients with hydatid liver cysts were operated since 1990 ?

\section{Follow-up}

14. Is there a follow-up programme after medical treatment?

$\square$ no

$\square$ yes if applicable, how often ...

15. Is there a follow-up programme after PAIR?

$\square$ no

$\square$ yes if applicable, how often ...

16. Is there a follow-up programme after surgery?

$\square$ no

$\square$ yes if applicable, how often ...

17. Approximately what is the percentage of follow-up programme after surgery?

18. What methods are used for follow-up?

$\square$ none 


\title{
Chapter 4
}

\author{
$\square$ ultrasonography \\ $\square$ CT scan \\ $\square \mathrm{MRI}$ \\ $\square$ serology
}

19. How many recurrences have occurred since 1990

$\square$ after PAIR ... recurrences

$\square$ after surgery ... recurrence

20. Are these numbers presented in the survey:

$\square$ estimated numbers

$\square$ actual numbers

\section{Evidence}

22. Based on the recent evidence, surgery remains primary treatment of choice

$\square$ yes

$\square$ no

$\square$ only for complicated cysts

\section{Incidence in your clinic}

23. In order to estimate the incidence per region, we would like to ask you the following questions:

a) Total treated patients with echinococcosis since 1990

b) Mean number of patients treated per year

c) Number of patients with solitary cysts per year

d) Number of patients with multiple cysts per year 


\section{Part 3}

Surgical management of benign solid liver lesions: hepatocellular adenomas 
Part 3 


\section{INTRODUCTION}

Hepatocellular adenomas are rare benign tumours with a prevalence of 3 per 100.000. ${ }^{1,2}$ These liverlesions are predominantly found in young females in their third or fourth decades taking oral contraceptives. The incidental finding of these tumours increased after the wide usage of oral contraceptive in the late seventies of the past century ${ }^{1,3}$. Not only was this benign lesion incidentally found by the increased use of the abdominal imaging. These tumours are also known because of the spontaneous rupture in the liver leading to life threatening haemorrhage ${ }^{4,5}$. At first, the treatment consisted of acute resection of the tumour with, however, a mortality of $5-10 \%{ }^{6}$. Gradually this aggressive therapy changed towards initial resuscitation and acute laparotomy and gauze packing without resection ${ }^{7}$. Some reports were noted of the embolisation of ruptures liver lesions ${ }^{4,5,8}$. Therefore, these life-threatening haemorrhages were treated in Leiden with heamodynamic support followed by acute selective arterial embolisation (chapter 7). The hypothesis was investigated whether this novel technique was safe and effective as first treatment.

Another complication of these benign liver adenomas is the malignant transformation. This is reported with an incidence of $10 \%$, however, proper evidence is lacking ${ }^{9}$. Therefore, systematic search for evidence was needed (chapter 8 ).

In this part the following questions were investigated:

1. Can selective arterial embolisation avoid invasive surgery when a ruptured adenoma is discovered during pregnancy?

2. Is selective arterial embolisation a good first approach in the treatment of life threatening haemorrhage of ruptured liver adenomas?

3. What is the reported incidence of malignant degeneration of hepatocellular adenomas?

\section{REFERENCES}

1. Rooks JB, Ory HW, Ishak KG, et al. Epidemiology of hepatocellular adenoma. The role of oral contraceptive use Jama 1979; 242: 644-648.

2. Reddy KR, Schiff ER. Approach to a liver mass Semin Liver Dis 1993; 13: 423-435.

3. Baum JK, Bookstein JJ, Holtz F, et al. Possible association between benign hepatomas and oral contraceptives Lancet 1973; 2: 926-929.

4. Ault GT, Wren SM, Ralls PW, et al. Selective management of hepatic adenomas Am Surg 1996; 62: 825-829.

5. Leese $\mathrm{T}$, Farges $\mathrm{O}$. Bismuth $\mathrm{H}$ Liver cell adenomas. A 12-year surgical experience from a specialist hepato-biliary unit Ann Surg 1988; 208: 558-564. 
Part 3

6. Eckhauser FE, Knol JA, Raper SE, et al. Enucleation combined with hepatic vascular exclusion is a safe and effective alternative to hepatic resection for liver cell adenoma Am Surg 1994; 60: 466471; discussion 472.

7. Terkivatan T, de Wilt JH, de Man RA, et al. Treatment of ruptured hepatocellular adenoma Br J Surg 2001; 88: 207-209.

8. Wheeler PG, Melia W, Dubbins $P$, et al. Non-operative arterial embolisation in primary liver tumours Br Med J 1979; 2: 242-244.

9. Barthelmes L, Tait IS. Liver cell adenoma and liver cell adenomatosis HPB (Oxford) 2005; 7: 186196. 


\section{Chapter 5}

\section{Life threatening haemorrhage from adenomas in the liver during pregnancy}

J.H.M.B. Stoot, J. van Roosmalen, O.T. Terpstra, A.F.M. Schaapherder

Adapted from Dig Surg 2006;23:155 
Chapter 5

\section{ABSTRACT}

An at term pregnant woman was admitted to a peripheral hospital with sudden right upper abdominal pain and diagnosed as having pre-eclampsia. Labour was induced and she was referred to our hospital because of increasing pain and decreasing haemoglobin levels. At admission ultrasound examination showed a possible liver haematoma and abdominal fluid. After delivery she remained hemodynamically instable and a contrast enhanced CT scan revealed four lesions resembling adenomas and free peritoneal fluid. The patient could not be stabilized with conservative treatment. A selective embolisation of the right hepatic artery was performed successfully. 


\section{INTRODUCTION}

Hepatocellular adenomas are rare benign liver tumours. They usually occur in young women and are often diagnosed after rupture. The reported increase in incidence after the introduction of oral contraceptives in the late seventies suggested that there might be a hormonal basis for the development of hepatocellular adenomas. ${ }^{1}$ This was strengthened by the reported reduction or complete regression of adenomas after cessation of estrogen use. ${ }^{2,3}$ During pregnancy, these tumours have been known to grow and due to increased vasculature and elevated levels of steroids, they seem more likely to rupture. ${ }^{4,5}$

We report a case of a pregnant woman with right upper quadrant pain and hemodynamic instability due to bleeding adenomas of the liver. The approach to diagnosis and treatment of this entity is discussed.

\section{Case report}

A healthy 30-year old primigravid woman with a term pregnancy was seen by her consulting gynaecologist complaining of progressive abdominal pain. Prior to admission, pregnancy had progressed without any complications. Physical examination showed right upper quadrant pain and a diastolic blood pressure of $95 \mathrm{mmHg}$. Her urine specimen was positive for protein. Ultrasound examination demonstrated a liver haematoma of $8 \mathrm{~cm}$ in diameter and a small amount of abdominal fluid. The patient was diagnosed as having pre-eclampsia and labour was induced by oxytocin infusion. She was referred to our hospital because of increasing pain and decreasing haemoglobin. Her laboratory tests showed low haemoglobin levels $(4.9 \mathrm{mmol} / \mathrm{L})$, normal platelets count $\left(220 \times 10^{9} / \mathrm{L}\right)$ and elevated liver enzymes (AST $158 \mathrm{U} / \mathrm{L}, \mathrm{ALT} 42 \mathrm{U} / \mathrm{L})$. One hour after admission, a healthy girl of 3875 gram was born by vacuum extraction.

Directly after birth, the patients systolic blood pressure dropped to $80 \mathrm{mmHg}$ with a pulse rate of $140 / \mathrm{min}$. Resuscitation was initiated, which included a blood transfusion of two packed cells (PC). Shortly after, the patient complained of progressive pain in the right upper abdomen and back despite analgesics. Physical examination of the abdomen showed tenderness in the right upper quadrant and muscular rigidity. Repeated ultrasound showed free peritoneal fluid and a tumorous process in the liver of $11.5 \mathrm{~cm}$. There was no fluid around the liver. An emergency contrast enhanced computed tomography (CT) scan showed four large lesions in the liver, most likely hepatocellular adenomas (Fig 1). The largest adenoma in segment $\mathrm{V}$ measured $10 \times 12 \mathrm{~cm}$ with signs of recent haemorrhaging. Other adenomas were seen in segment VI and VII. One lesion was suspect for bleeding into the abdominal cavity. The patient could not be stabilized non-operatively. A selective embolisation of the right arteria hepatica was successfully conducted in order 
to stabilize the patient (Fig 2). She recovered swiftly and both mother and daughter were discharged two weeks later. Since it is commonly accepted that estrogen promotes adenoma growth, she was advised to refrain from oral contraconceptives. An intra-uterine device was placed at her postnatal check-up. Follow-up CT scan 2 months after embolisation showed complete necrosis of the large lesion in the right lobe and resorption of the haematoma. Eleven months after admission, the patients CT scan showed a progressive decrease in size of all tumours. The largest tumour was reduced to $7 \times 9.5 \mathrm{~cm}$. In this case, we will conduct further radiological follow up to observe the size of the adenomas. If one of the lesions will enlarge, we will perform an elective resection to prevent malignant degeneration.

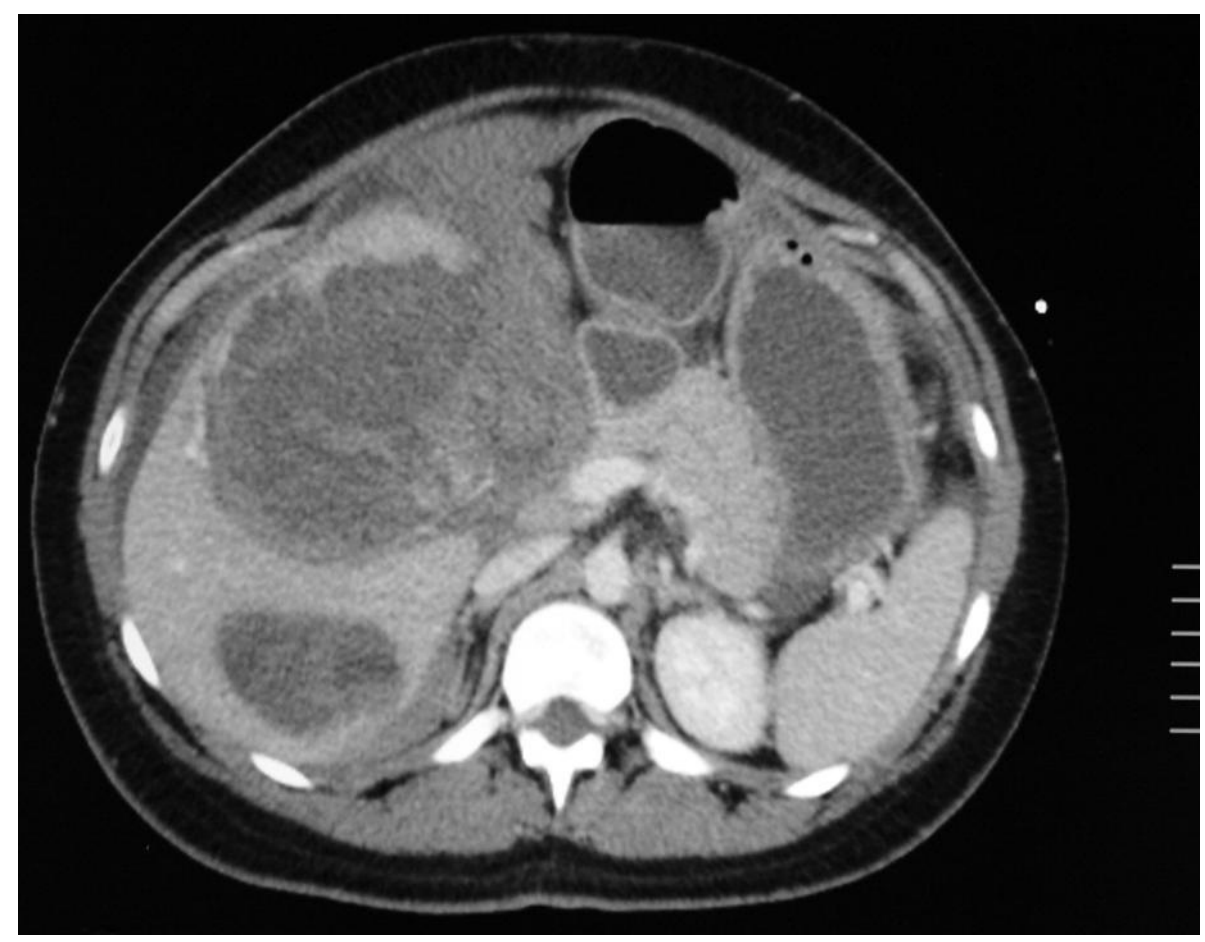

Fig. 1. Contrast enhanced computed tomography scan of the liver showing several adenomas; the largest in segment $\mathrm{V}(10 \mathrm{~cm})$, with signs of recent bleeding. The other adenomas are in segment $\mathrm{VI}$ and VII, one suspected for recent bleeding into the abdominal cavity. 


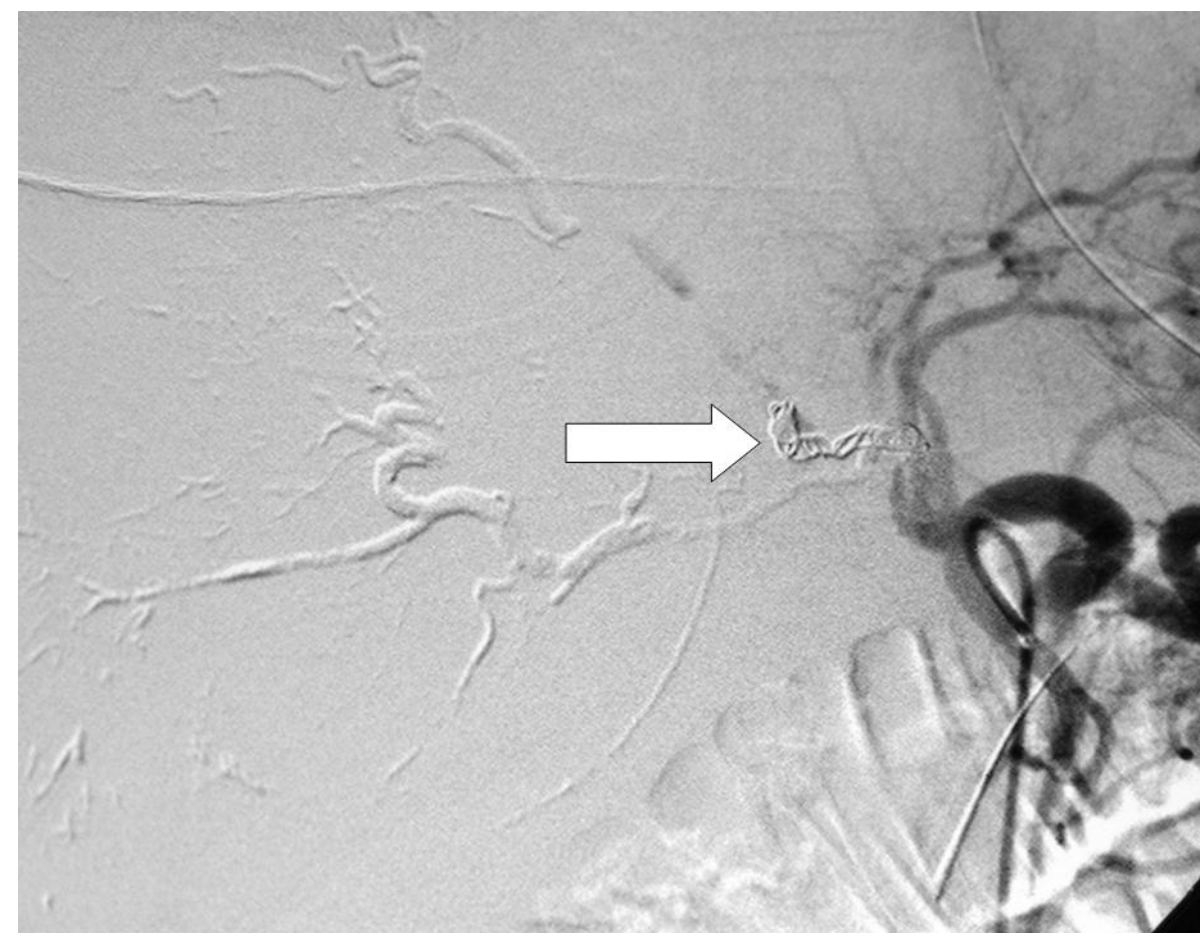

Fig. 2. Angiography of the liver after selective embolisation of the right hepatic artery. Contrast fills the common hepatic artery and the left hepatic artery. Note the used coils in the origin of the right hepatic artery (arrow).

\section{DISCUSSION}

Liver rupture during childbirth is a serious and potential mortality risk for pregnant women and is predominantly associated with either atypical pre-eclampsia or HELLP syndrome. Haemorrhage in pregnancy from liver adenomas is rare and therefore easily misdiagnosed. ${ }^{6}$ Presentation may be with severe pain localised to the epigastrium or right upper quadrant with radiation to the back or right shoulder. These symptoms might be misinterpreted as dyspepsia, cholecystitis or anxiety. Delay in diagnosing rupture of the liver will contribute to high mortality rates. Liver rupture due to HELLP syndrome and bleeding from liver adenomas are different entities and require different therapeutic strategies. In order to visualize adenomas and potential bleeding, it is essential to perform a CT scan.

Controversy remains upon emergency management for ruptured adenomas. Acute surgical resection is advised as preferred treatment by many authors, ${ }^{6}$ despite an associated mortality rate up to $8 \% .^{7}$ In contrast, some authors have suggested a more conservative approach with primarily hemodynamical stabilization, 
followed by preventive partial liver resection for tumours exceeding five $\mathrm{cm}$ because of potential malignant degeneration. ${ }^{5}$ Arterial embolisation was proposed before as selective treatment for bleeding adenomas. ${ }^{2}$ To the best of our knowledge, no series of patients with bleeding adenomas and selective embolisation have been reported until now. In this report we show that selective embolisation might be a valuable tool to control severe bleeding from ruptured adenomas in the liver. Also, this case emphasizes the need for a high degree of suspicion for liver adenomas when women present with right upper abdominal pain during pregnancy.

\section{REFERENCES}

1. Klatskin G. Hepatic tumors: possible relationship to use of oral contraceptives. Gastroenterology 1977; 73: 386-94.

2. Ault GT, Wren SM, Ralls PW, et al. Selective management of hepatic adenomas. Am Surg 1996; 62: 825-829.

3. Nagorney DM. Benign hepatic tumors: focal nodular hyperplasia and hepatocellular adenoma. World J Surg 1995; 19: 13-8.

4. Kent DR, Nissen ED, Nissen SE, et al. Effect of pregnancy on liver tumor associated with oral contraceptives. Obstet Gynecol 1978; 51: 148-151.

5. Terkivatan T, de Wilt JH, De Man RA, et al. Management of hepatocellular adenoma during pregnancy. Liver 2000; 20: 186-187.

6. Cobey FC, Salem RR. A review of liver masses in pregnancy and a proposed algorithm for their diagnosis and management. Am J Surg. 2004; 187: 181-191.

7. Eckhauser FE, Knol JA, Raper SE, et al: Enucleation combined with hepatic vascular exclusion is a safe and effective alternative to hepatic resection for liver cell adenoma. Am.Surg 1994; 60: 46671. 


\section{Chapter 6}

\section{Necrosis of a large hepatic tumour after haemorrhage and subsequent selective arterial embolisation}

V.A.L. Huurman, J.H.M.B. Stoot, E. van der Linden, O.T.Terpstra,

A.F.M. Schaapherder

Adapted from World J Gastroenterol, 2006;12[37], 6059-6061 
Chapter 6

\section{ABSTRACT}

This case report describes a young female patient presenting with acute intraabdominal hemorrhage originating from a large tumor in the liver, most likely a hepatocellular adenoma. The haemorrhage was stopped by selective embolisation of a right hepatic artery branch. Subsequently, partial hepatectomy was performed after 6 months. Macro- and microscopic examination showed complete necrosis and absence of tumorous tissue. The patient was discharged without complications, and subsequent follow-up until 22 months after resection did not reveal any new lesions in the liver. This case emphasizes the significance of selective arterial embolisation in the management of haemorrhaging liver tumours, and questions the need for (partial) hepatectomy after this procedure in selective cases. 


\section{INTRODUCTION}

Hepatocellular adenomas are uncommon benign liver tumours that are present mainly in women of reproductive age. ${ }^{1}$ Their existence is associated with the use of oral contraceptives. ${ }^{2}$ The first clinical presentation of the tumour can consist of abdominal pain, hepatomegaly, or hepatic (subcapsular) haemorrhage and shock $^{1}$ For the latter presentation, emergency surgery by partial hepatectomy is the current treatment of choice. However, this treatment is associated with high morbidity and mortality rates. ${ }^{3,4}$ Therefore, several groups suggest a role for initial conservative treatment ${ }^{1,5}$ Selective arterial embolisation is a novel method for the management of intra-abdominal haemorrhage without the need for major surgery, and our hospital has gained considerable experience with this procedure. However, the use of this elegant method when coping with liverhaemorrhage has only been described incidentally. ${ }^{1,5,6}$

After any initial emergency treatment, secondary partial hepatectomy is generally recommended in patients with lesions $\geq 5 \mathrm{~cm}$, because of increased risk of rupture as well as malignant degeneration. ${ }^{1,5}$ The case we present, however, suggests a role of selective arterial embolisation not only in the primary emergency situation, but also as a therapeutic tool to achieve necrosis of an arterially vascularized liver tumour.

\section{CASE REPORT}

A 35-year-old woman was referred to our hospital with a two-day history of right abdominal pain without any previous trauma. The patient had no medical history, her medication consisted of oral anticonceptive treatment only (30 $\mu \mathrm{g}$ ethinylestradiol, $150 \mu \mathrm{g}$ levonorgestrel, taken during eleven years). Despite initial fluid suppletion, her heart rate was elevated $(110 / \mathrm{min})$ with normal blood pressure. Further physical examination showed tenderness of the right upper abdomen. Hemoglobin levels were low (4.9 mMol/I). INR was 1.0, APTT $21.5 \mathrm{~s}$, PTT $12.5 \mathrm{~s}$ and thrombocytes $213 \times 10^{9} / \mathrm{L}$. Serum liver enzyme levels were elevated (ASAT $550 \mathrm{U} / \mathrm{I}$, ALAT 607 U/I, Alk.Fos 75 U/I, yGT 109 U/I, LDH 758 U/I). She was transfused with 2 units of red blood cells. Computed tomography (CT) scan of the abdomen revealed a large subcapsular haematoma surrounding a tumorous mass in segment IV-VII of the liver, as well as intraperitoneal fluid. Arteriography confirmed haemorrhage originating from the right hepatic artery, which was also responsible for the vascularisation of the tumour.

Subsequently, selective arterial embolisation of the majority of right hepatic artery branches was performed in order to stop the haemorrhage, using both polyvinyl alcohol (PVA) particles and a mixture of enbucrilate (Histoacryl) and iodized 
poppy-seed oil (Lipiodol). Contrast-enhanced CT scan 6 days after embolisation clearly showed a circumscribed tumorous mass $(\varnothing 7 \mathrm{~cm})$ in segment $V$ of the liver, most likely a hepatocellular adenoma (Figure 1).. Further hospital stay was unremarkable and the patient was discharged 11 days after admission.

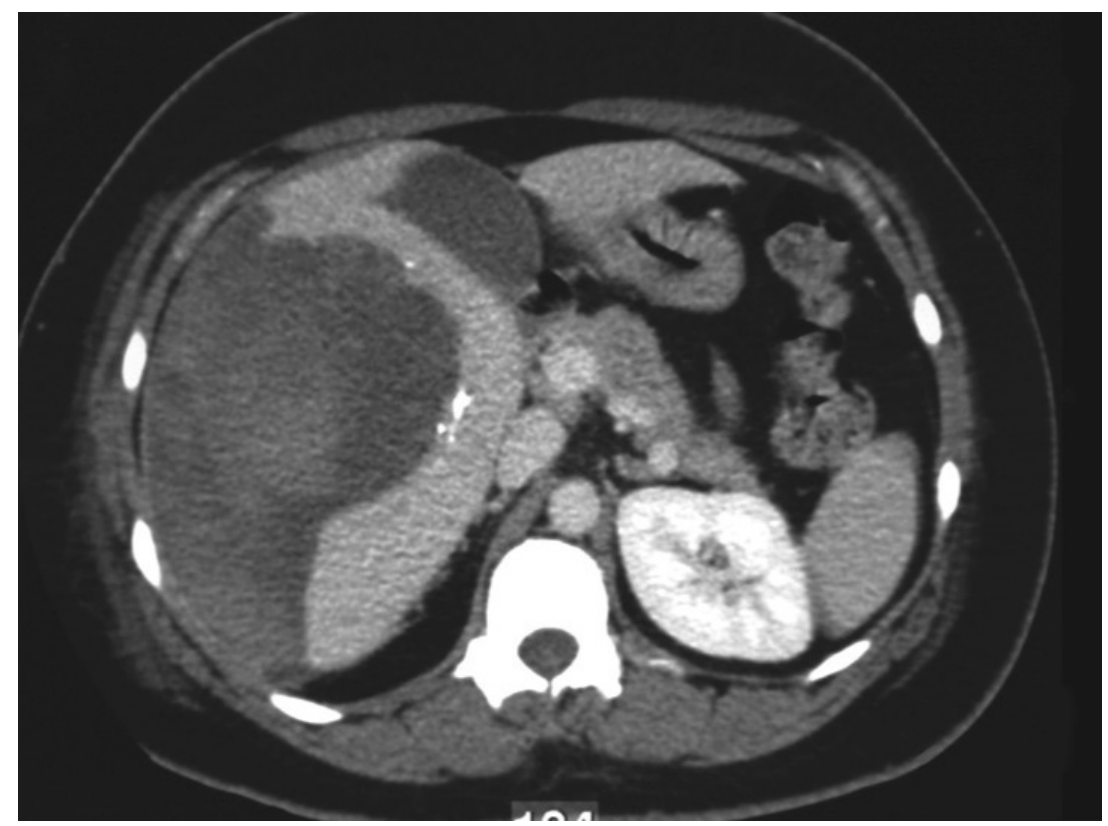

Figure 1. Contrast-enhanced computed tomography (CT) scan of the abdomen 6 days after selective arterial embolisation, showing liver haemorrhage with a circumscribed mass as its conceivable origin.

Following our department's protocol on liver adenomas with a diameter $\geq 5 \mathrm{~cm}$, elective resection of segment $\mathrm{V}$ was performed six months later. Preoperative contrast-enhanced CT scan showed the remains of both the hematoma and the known circumscript mass (Figure 2). Arterial perfusion of segment $V$ of the liver was absent whereas portal perfusion was intact. The resected tumor consisted completely of necrotic material (Figure 3). No clear tumour classification could be made by histological examination due to absence of tumorous material, but the macroscopic signs of necrosis were confirmed. 


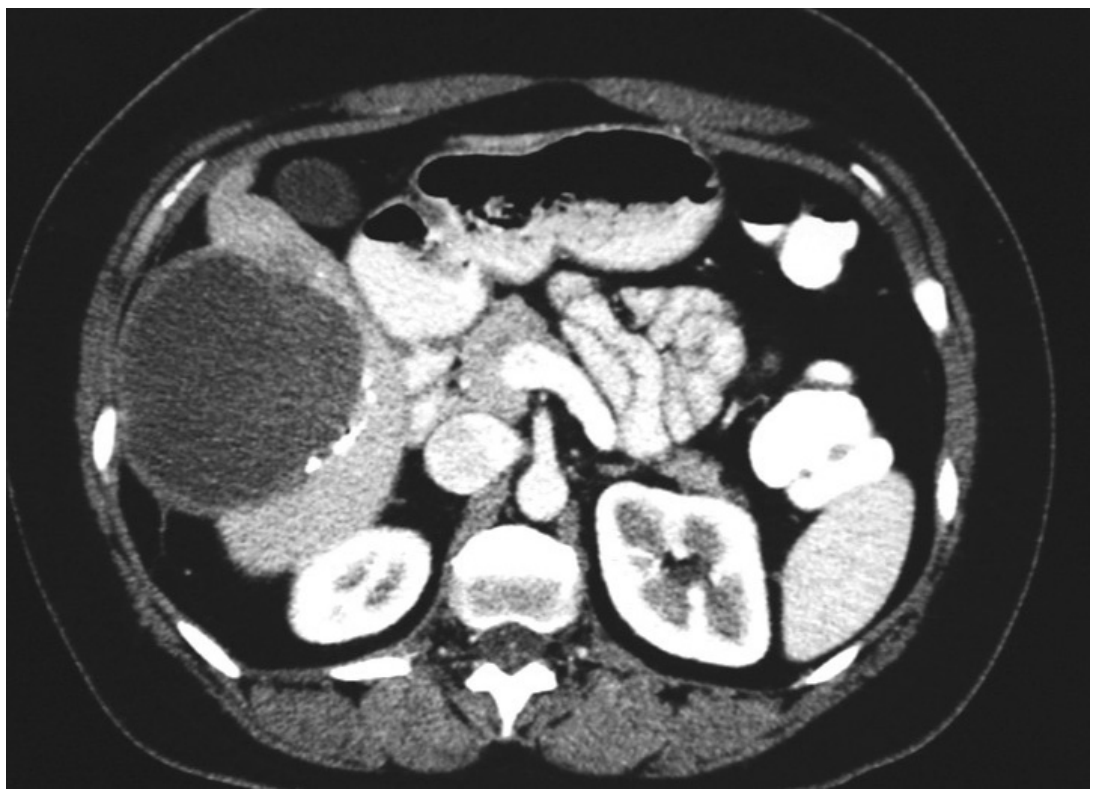

Figure 2. Arterial phase CT scan of the abdomen before partial hepatectomy, showing remains of the hematoma and the known circumscribed mass, which is not vascularised arterially. Note the arterial deposition of Histoacryl en Lipiodol along the tumour as a result of the selective embolisation.

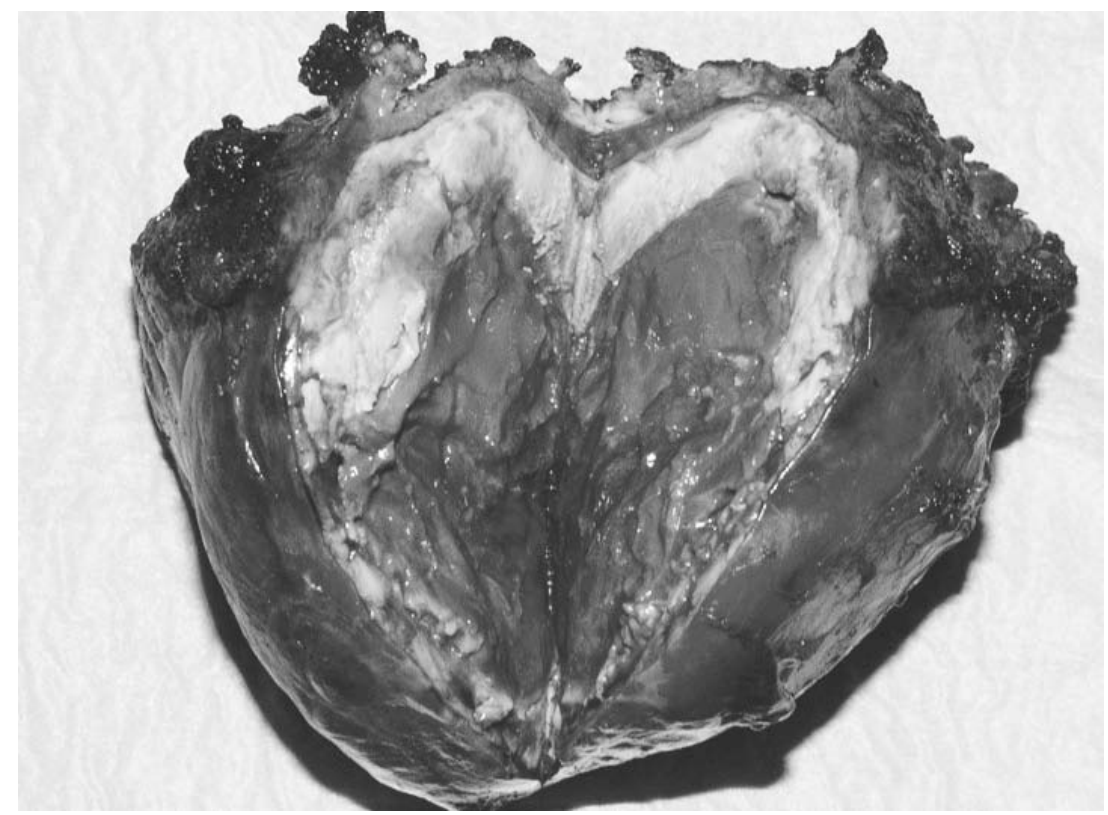

Figure 3. Macroscopic view of the resected tumour $(\varnothing 7 \mathrm{~cm})$, consisting entirely of necrotic material. 
Tumour markers (CEA, CA 19.9 and $\alpha$-foetoprotein) were in the normal range. The patient was discharged without complications, and subsequent follow-up until 22 months after resection did not reveal any new lesions in the liver. She was advised to refrain from oral contraceptive medication.

\section{DISCUSSION}

The increased chance of rupture of large hepatocellular adenomas makes the presence of such a tumour a considerable potential hazard. Management of this condition still needs improvement. The presence of a ruptured hepatocellular adenoma cannot be proven by CT scan alone, but is strongly suggested by our patient's clinical presentation. Although hepatocellular carcinoma and focal nodular hyperplasia lesions have also been described as causes of liver haemorrhage, ${ }^{7}$ their presence is unlikely in this patient. The presence of liver adenomas in young women is associated with the use of oral contraceptives. In this case no specific histologic tumourclassification could be made, since cessation of arterial blood flow by embolisation caused necrosis of the tumor. This corresponds with the fact that hepatocellular adenomas are only vascularized arterially. It has to be noted that regression of liver adenomas is possible not only after ischemia, but also after hormone withdrawal, ${ }^{8}$ hemodialysis ${ }^{9}$ and dietary therapy for glycogen storage disease. ${ }^{10}$

Embolisation is safe and successful in stopping the haemorrhage. The necrosis of the tumour may point to a future role of selective arterial embolisation in the management of either haemorrhaging or non-haemorrhaging hepatocellular adenomas. Furthermore, this case argues in favour of conservative follow-up after embolisation, regardless of the initial size of the adenoma. This would further limit the indications for surgery in a selected group of patients, resulting in reduction of patient morbidity and mortality.

\section{REFERENCES}

1. Ault GT, Wren SM, Ralls PW, et al. Selective management of hepatic adenomas. Am Surg 1996; 62: 825-829.

2. Rooks JB, Ory HW, Ishak KG, et al. Epidemiology of hepatocellular adenoma. The role of oral contraceptive use. JAMA 1979; 242: 644-648.

3. Shortell CK, Schwartz SI. Hepatic adenoma and focal nodular hyperplasia. Surg Gynecol Obstet 1991; 173: 426-431.

4. Leese $\mathrm{T}$, Farges $\mathrm{O}$, Bismuth $\mathrm{H}$. Liver cell adenomas. A 12-year surgical experience from a specialist hepato-biliary unit. Ann Surg 1988; 208: 558-564.

5. Terkivatan T, de Wilt JH, de Man RA, et al. Indications and long-term outcome of treatment for benign hepatic tumors: a critical appraisal. Arch Surg 2001; 136: 1033-1038. 
6. Wheeler PG, Melia W, Dubbins P, et al. Non-operative arterial embolisation in primary liver tumours. Br Med J 1979; 2: 242-244.

7. Kleespies A, Settmacher U, Neuhaus P. Spontaneous rupture of hepatic focal nodular hyperplasia-a rare cause of acute intraabdominal bleeding. Zentralbl Chir 2002; 127: 326-328.

8. Aseni P, Sansalone CV, Sammartino C, et al. Rapid disappearance of hepatic adenoma after contraceptive withdrawal. J Clin Gastroenterol 2001; 33: 234-236.

9. lijima H, Moriwaki $\mathrm{Y}$, Yamamoto $\mathrm{T}$, et al. Spontaneous regression of hepatic adenoma in a patient with glycogen storage disease type I after hemodialysis: ultrasonographic and CT findings. Intern Med 2001; 40: 891-895.

10. Parker P, Burr I, Slonim A, et al. Regression of hepatic adenomas in type la glycogen storage disease with dietary therapy. Gastroenterology 1981; 81: 534-536. 



\section{Chapter 7}

\section{Life saving therapy for haemorrhaging liver adenomas using selective arterial embolisation}

J.H.M.B. Stoot, E. van der Linden, O.T. Terpstra, A.F.M. Schaapherder

Adapted from British Journal of Surgery, 2007; 94:1249-53.

(Schoemaker Award 2007)

Presented to the Annual Scientific Meeting of the Association of Surgeons of Great Britain and Ireland, Edinburgh, Scotland, May 2006, and presented to the $7^{\text {th }}$ World Congress of the International Hepato-Pancreato Biliary Association, Edinburgh, Scotland, September 2006. 


\section{ABSTRACT}

Background: Emergency treatment for patients with a ruptured hepatocellular adenoma is controversial. The aim of this study was to evaluate the management of ruptured adenomas with selective arterial embolisation.

Methods: The study included 11 consecutive patients treated for ruptured hepatocellular adenomas in the period 2001-2006. After initial hemodynamic support, all patients received selective embolisation of branches of the hepatic artery. The primary outcome was effectiveness in stopping the haemorrhage. Secondary outcomes were complications and changes in tumour size after embolisation.

Results: A single embolisation brought haemorrhaging under control in ten patients; one patient needed three embolisations. None of the patients required emergency surgery. In the follow-up of 19 months (range 7-49 months) no general complications or hepatobiliary complications were observed. All 25 adenomas, including those without signs of haemorrhaging in the same liver lobe were either smaller or not detectable on computed tomography or magnetic resonance imaging after embolization, with the median diameter decreasing from $7.0 \mathrm{~cm}$ to $2.5 \mathrm{~cm}$ $(P<0.001)$.

Conclusions: Selective embolisation of the hepatic artery is a save and adequate first approach in the management of patients with haemorrhaging hepatocellular adenomas. Furthermore, arterial embolisation reduces the size of adenomas in the liver. 


\section{INTRODUCTION}

Although emergency surgery for ruptured hepatocellular adenomas of the liver is associated with high mortality and morbidity rates, it remains the treatment of choice. ${ }^{1,2}$ Emergency resection of ruptured adenomas has mortality rates between 5 and 10 per cent, whereas elective surgery has a mortality rate of less than 1 per cent. ${ }^{3-5}$ A recent study, showed that conservative management of ruptured hepatocellular adenoma may be a good alternative treatment, as none of the 12 patients died. ${ }^{6}$ Unfortunately, three of those patients required urgent laparotomy and gauze packing because of life-threatening haemorrhaging. Selective arterial embolisation for ruptured hepatocellular adenomas may be a valuable alternative, although reports of this are rare. ${ }^{7-10}$ In Leiden University Medical Centre, selective embolisation of hepatic artery branches is performed as initial therapy to stabilise hemodynamically unstable patients with haemorrhaging ruptured hepatocellular adenoma.

The aim of this study was to evaluate the management of ruptured hepatocellular adenomas using selective arterial embolisation.

\section{METHODS}

The study included 11 consecutive patients were treated for ruptured hepatocellular adenoma (ten women, one man; mean age 33.5 (range: 18-43 years) between January 2001 and January 2006). Nine of the women had a history of oral anticonceptive use during several years. Seven patients had more than one ruptured adenoma. In all but one patient, the adenomas were located in the right hepatic lobe (median diameter $7 \mathrm{~cm}$, range 0.7-15 cm). Laboratory tests on serum CA19-9, carcinoembryonic antigen and $\alpha$-fetoprotein were normal in all patients. Distinction of the liver lesion was made by ultrasonography, computed tomography (CT) scanning and/or gadolinium and superparamagnetic iron oxide (SPIO)-enhanced magnetic resonance imaging (MRI). ${ }^{11}$ Selective embolisation was performed with polyvinyl alcohol particles and coils or using a mixture of enbucrilate (Histoacry| ${ }^{\circledR}$; B.Braun Aesculap, Tuttlingen, Germany) and iodized poppy-seed oil (Lipiodol). Contrastenhanced CT showed characteristics of the adenomas: hepatic tumours with arterial perfusion starting at the periphery with centripetal progression. Gadolinium and SPIO-enhanced MRI were used also during follow-up. In one patient there was some doubt about the diagnosis, and a liver biopsy was performed. Histopathological examination confirmed hepatocellular adenoma.

The primary outcome was defined as the success of embolisation therapy: hemodynamic stabilisation and bleeding stopped. Failure of the initial therapy was defined as hemodynamically instability after embolisation warranting emergency 
surgery. Secondary outcomes were morbidity (minor and major complications) and changes in size of haemorrhaging and non-haemorrhaging adenomas after selective embolization. Complications were defined as minor (generally non-lifethreatening or nosocomial) or major (intra-abdominal abscess, sepsis, major haemorrhaging requiring new interventions or death).

Changes in adenoma size were measured during radiological follow-up and compared with initial measurements. Measurements before and after embolization were compared using the paired Student's $t$ test. $P<0.050$ was considered significant.

\section{RESULTS}

In all 11 patients, haemorrhaging was the first presentation of hepatocellular adenoma. In total, 23 units of red blood cells were required to stabilize these patients (mean haemoglobin level 5.8(range 3.6-8.9) $\mathrm{mmol} / \mathrm{l}$,). Four units were given after embolization for the whole group. Eight patients presented with hemodynamic shock (class I-III). Seven patients were referred to the authors' centre after initial hemodynamic support and three were stable at admission. All but two of the patients haemorrhaged into the abdominal cavity. After initial haemodynamic support, all 11 patients had selective embolisation of one ore more branches of the hepatic artery: the right hepatic artery in seven patients, the left hepatic artery in one, the combined right and left hepatic artery in two and the common hepatic artery in one (because of dissection of this artery during the procedure). None of the patients required secondary emergency surgery. One patient required two additional embolisations because of a false aneurysm close to the necrosis caused by the primary embolisations.

In four patients, only minor complications were observed, consisting of fever without other clinical symptoms of infection. One patient developed pleural effusion, which was aspirated. This procedure was complicated by a pneumothorax. No major complications arose from the embolisation itself.

In the first two patients, elective surgical segmental liver resection was carried out because of adenoma size $(7$ and $15 \mathrm{~cm}$ ), in accordance with the guidelines for patients with large adenomas. Histopathological examination showed complete necrosis of these adenomas, consistent with follow-up CT. so, the decision was taken not to resect tumours larger than $5 \mathrm{~cm}$ after embolisation but to ensure frequent radiological follow-up. In the remaining nine patients, follow-up CT or MRI consistently showed tumour necrosis and resorption after embolisation. Moreover, both haemorrhaging and non-haemorrhaging hepatocellular adenomas shrank during follow-up in all patients (Fig. 1). The median decrease in size was $5.0 \mathrm{~cm}(P<$ 
0.001) (Figure 2); the difference in size was significant even when haemorrhging and non-haemorrhaging adenomas were analysed separately (Table 1).

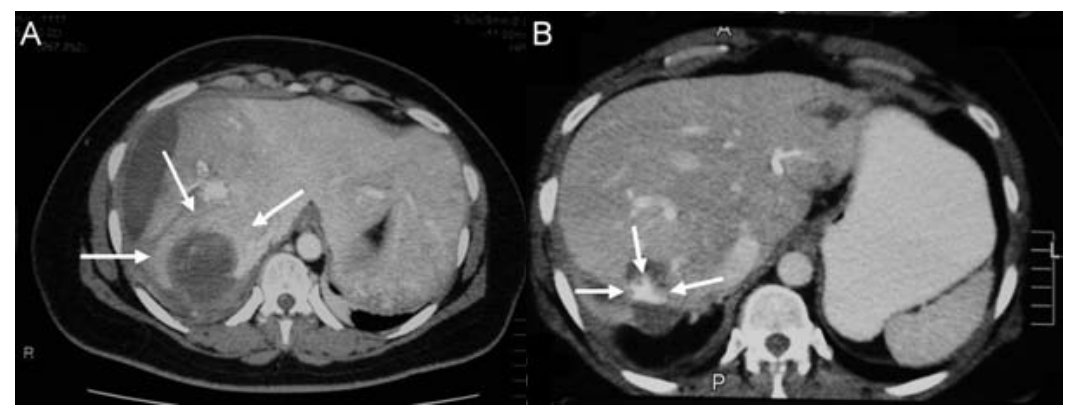

A. Before embolisation

B. After embolisation (17months)

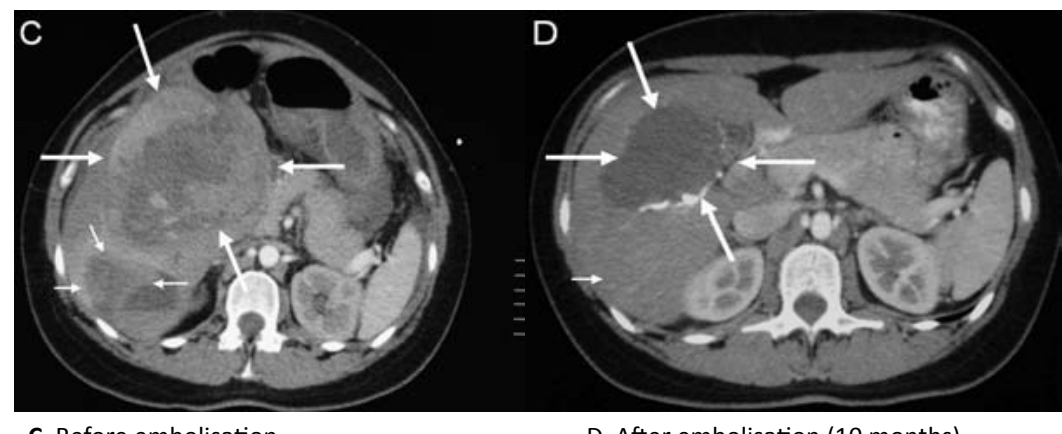

C. Before embolisation

D. After embolisation (10 months)

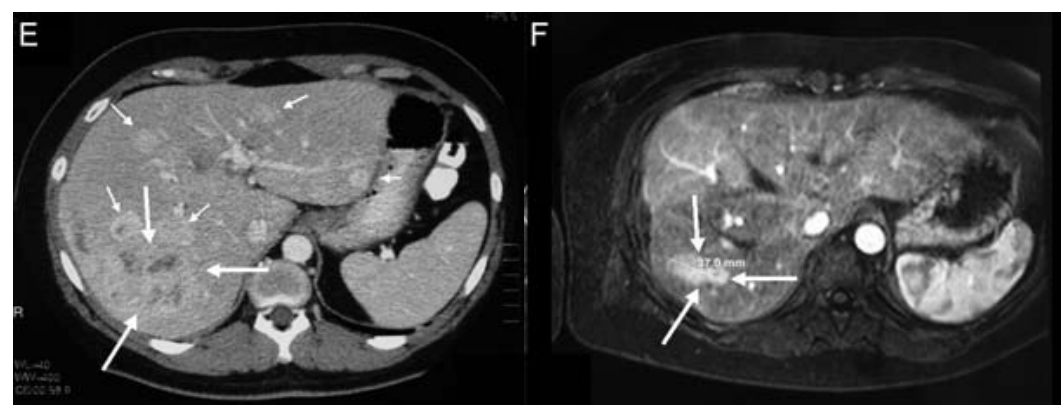

E. Before embolisation

F. After embolisation (36 months)

Figure 1. Typical examples of hepatocellular adenomas during radiological follow-up. (A) Contrast enhanced computed tomography (CT) scan of the liver showing one large adenoma $(7 \mathrm{~cm})$ with signs of bleeding. (B) CT scan 17 months after embolisation; the adenoma measures $2 \mathrm{~cm}$. (C) CT scan in a patient with several adenomas; the largest in segment $\mathrm{V}$ (large arrows), measuring $10 \mathrm{~cm}$, and showed signs of recent bleeding. The other adenomas are in segment VI and VII, one suspected for recent bleeding into the abdominal cavity (small arrows). (D) CT scan 10 months after embolisation; the largest adenoma measures $5 \mathrm{~cm}$. (E) CT scan showing several adenomas in a patient with adenomatosis (small arrows) and a large bleeding adenoma in segment VII-VIII (large arrow). (F) Follow-up Magnetic Resonance Imaging scan 35 months after embolisation; most of the adenomas were no longer detectable. 


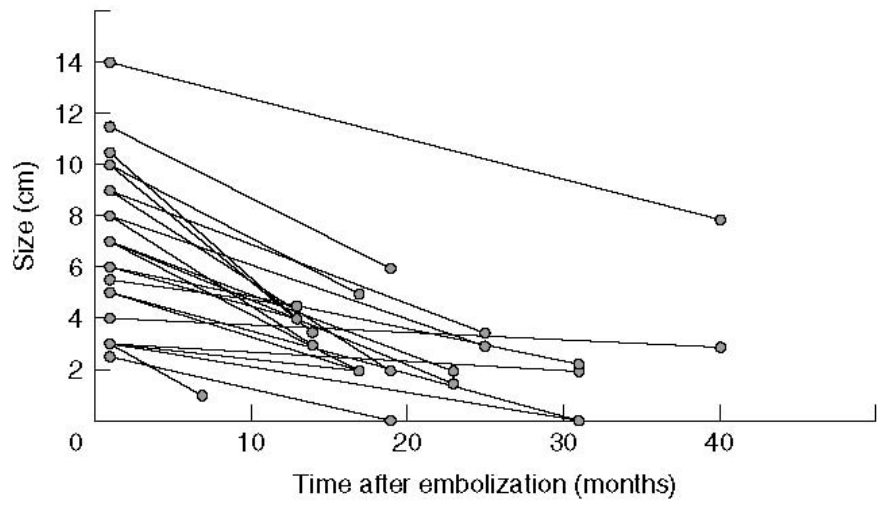

Figure 2. Changes in size of hepatocellular adenomas after selective arterial embolisation as measured during radiological follow-up. All adenomas decreased in size (median decrease in size was 5.0 (range 7.0-1.0) $\mathrm{cm}(P<0.001$, paired Student's $t$ test)

Table 1 The decrease in size of haemorrhaging and non-haemorrhaging hepatocellular adenomas after selective arterial embolisation.

\begin{tabular}{llll}
\hline Adenomas & $\begin{array}{l}\text { Size before embolisation } \\
(\mathrm{cm})\end{array}$ & Size after embolisation $(\mathrm{cm})$ & $P$-value* \\
\hline Haemorrhaging $(\mathrm{n}=17)$ & 8.0 & 3.0 & $<0.001$ \\
non-haemorrhaging $(\mathrm{n}=8)$ & 3.5 & 2.0 & $<0.004$ \\
All adenomas $(\mathrm{n}=25)$ & 7.0 & 2.5 & $<0.001$ \\
\hline
\end{tabular}

All values are medians. *Paired Student's $t$ test.

No general or hepatobiliairy complications, such as bile leakage or ischaemic strictures of bile ducts, were observed during follow-up (median 19 (range 7-49) months).

\section{DISCUSSION}

This study found selective arterial embolisation for ruptured and haemorrhaging hepatocellular adenomas to be a safe and effective method of haemodynamic stabilization. None of the 11 patients required surgery and only one required additional embolisations. There were a few minor complications including fever, probably caused by tissue necrosis after embolisation. Although the authors had anticipated biliairy complications, especially after dissection of the common hepatic artery, to date no biliairy stenosis or leakage has been observed in these patients. 
Size of hepatocellular adenomas is an established risk factor of malignancy ${ }^{7,9,12}$. Surgical resection has been recommended for adenomas with an initial diameter of $5 \mathrm{~cm}$ or more or in symptomatic patients, even during pregnancy ${ }^{6,13}$. The resection of large adenomas has also been recommended because the risk of haemorrhaging is relatively high. For smaller and asymptomatic adenomas, an expectant approach is advocated ${ }^{14}$.

The results of this study show that selective embolisation prevents growth of hepatocellular adenomas, including non-ruptured adenomas in patients with multiple adenomas. Hepatic adenoma regression has been reported before, but only after discontinuation of oral contraceptives ${ }^{15-17}$, and some reports have shown no change in the size of hepatocellular adenomas after oral contraceptives withdrawal $^{18,19}$.

The effect of embolization on a hepatocellular adenoma, an extensive proliferation of blood-filled sinusoids is understandable in the light of its morphological arterial blood supply, as observed with different imaging techniques ${ }^{20,21}$. This might also explain the disappearance of adenoma tissue after embolisation. Although the present study was not set up to evaluate the effect of embolisation on hepatocellular adenomas growth, all the lesions regressed after embolisation, even in the two patients who had not had oral contraceptives. Some adenomas were no longer detectable at all after embolisation in patients with multiple adenomas. However, initial measurements could have been clouded by the haematoma and consequent resorption in some cases. Selective embolisation might therefore be an option for large or symptomatic adenomas in a selected group of patients. As it avoids invasive surgery, it would also be suitable for pregnant women ${ }^{22}$.

Long-term follow-up of these patients is necessary, with repeated contrastenhanced CT- or MRI to discriminate between residual haematoma, cystic formation and residual liver tumour. Increase in tumour size should lead to a more aggressive approach including biopsy, ablation or surgical resection.

\section{Conclusions}

Selective hepatic artery embolisation is a valuable tool in the treatment of haemorrhaging hepatic adenomas. It might be considered as the first treatment of choice, avoiding emergency surgery, thus reducing the risk of surgeryrelated morbidity and mortality. 


\section{REFERENCES}

1. Cobey FC, Salem RR. A review of liver masses in pregnancy and a proposed algorithm for their diagnosis and management. Am J Surg 2004; 187: 181-191.

2. Eckhauser FE, Knol JA, Raper SE, Thompson NW. Enucleation combined with hepatic vascular exclusion is a safe and effective alternative to hepatic resection for liver cell adenoma. Am Surg 1994; 60: 466-471.

3. Belghiti J, Pateron D, Panis Y, Vilgrain V, Flejou JF, Benhamou JP et al. Resection of presumed benign liver tumours. Br J Surg 1993; 80: 380-383.

4. Flowers BF, McBurney RP, Vera SR. Ruptured hepatic adenoma. A spectrum of presentation and treatment. Am Surg 1990; 56: 380-383.

5. Shortell CK, Schwartz SI. Hepatic adenoma and focal nodular hyperplasia. Surg Gynecol Obstet 1991; 173: 426-431.

6. Terkivatan T, de Wilt JH, de Man RA, van Rijn RR, Tilanus HW, IJzermans JN. Treatment of ruptured hepatocellular adenoma. Br J Surg 2001; 88: 207-209.

7. Ault GT, Wren SM, Ralls PW, Reynolds TB, Stain SC. Selective management of hepatic adenomas. Am Surg 1996; 62: 825-829.

8. Huurman VA, Stoot JH, van der Linden E, Terpstra OT, Schaapherder AF. Necrosis of a large hepatic tumor after hemorrhage and subsequent selective arterial embolization. World J Gastroenterol 2006; 12: 6059-6061.

9. Leese $\mathrm{T}$, Farges $\mathrm{O}$, Bismuth $\mathrm{H}$. Liver cell adenomas. A 12-year surgical experience from a specialist hepato-biliary unit. Ann Surg 1988; 208: 558-564.

10. Wheeler PG, Melia W, Dubbins P, Jones, B, Nunnerley H, Johnson P et al. Non-operative arterial embolisation in primary liver tumours. Br Med J 1979; 2: 242-244.

11. Hussain SM, van den Bos I, Dwarkasing RS, Kuiper JW, den Hollander J. Hepatocellular adenoma: findings at state-of-the-art magnetic resonance imaging, ultrasound, computed tomography and pathologic analysis. Eur Radiol 2006; 16: 1873-1886.

12. Weimann A, Ringe B, Klempnauer J, Lamesch P, Gratz KF, Prokop M et al. Benign liver tumors: differential diagnosis and indications for surgery. World J Surg 1997; 21: 983-990.

13. Terkivatan T, de Wilt JH, de Man RA, IJzermans JN. Management of hepatocellular adenoma during pregnancy. Liver 2000; 20: 186-187.

14. Terkivatan T, de Wilt JH, de Man RA, van Rijn RR, Zondervan PE, Tilanus HW et al. Indications and long-term outcome of treatment for benign hepatic tumors: a critical appraisal. Arch Surg 2001; 136: 1033-1038.

15. Aseni P, Sansalone CV, Sammartino C, Benedetto FD, Carrafiello G, Giacomoni A et al. Rapid disappearance of hepatic adenoma after contraceptive withdrawal. J Clin Gastroenterol 2001; 33: 234236.

16. Buhler H, Pirovino M, Akobiantz A, Altorfer J, Weitzel M, Maranta E et al. Regression of liver cell adenoma. A follow-up study of three consecutive patients after discontinuation of oral contraceptive use. Gastroenterology 1982; 82: 775-782.

17. Kawakatsu M, Vilgrain V, Erlinger S, Nahum H. Disappearance of liver cell adenoma: CT and MR imaging. Abdom Imaging 1997; 22: 274-276.

18. Mariani AF, Livingstone AS, Pereiras RV, Jr., van Zuiden PE, Schiff ER. Progressive enlargement of an hepatic cell adenoma. Gastroenterology 1979; 77: 1319-1325.

19. Marks WH, Thompson N, Appleman H. Failure of hepatic adenomas (HCA) to regress after discontinuance of oral contraceptives. An association with focal nodular hyperplasia (FNH) and uterine leiomyoma. Ann Surg 1988; 208: 190-195.

20. Van Hoe L, Baert AL, Gryspeerdt S, Vandenbosh G, Nevens F, Van Steenbergen W et al. Dual-phase helical CT of the liver: value of an early-phase acquisition in the differential diagnosis of noncystic focal lesions. AJR Am J Roentgenol 1997; 168: 1185-1192. 
21. Welch TJ, Sheedy PF, Johnson CM, Stephens DH, Charboneau JW, Brown ML et al. Focal nodular hyperplasia and hepatic adenoma: comparison of angiography, CT, US, and scintigraphy. Radiology 1985; 156: 593-595.

22. Stoot JH, van Roosmalen J, Terpstra OT, Schaapherder AF. Life-Threatening Hemorrhage from Adenomas in the Liver during Pregnancy. Dig Surg 2006; 23: 155. 



\section{Chapter 8}

\section{Malignant transformation of hepatocellular adenomas into hepatocellular carcinomas: a systematic review including more than 1600 adenoma cases}

J.H.M.B. Stoot, R.J.S. Coelen, M.C. de Jong, C.H.C. Dejong

Adapted from HPB. 2010;12(8):509-22

Presented to the 9th World Congress of the International Hepato-Pancreato-Biliary Association in Buenos Aires, Argentina, April 2010. 


\section{ABSTRACT}

Background: Malignant transformation of hepatocellular adenomas (HCAs) into hepatocellular carcinomas (HCCs) has been reported repeatedly and is considered one of the main reasons for surgical treatment. However, its actual risk is currently unknown. Aim of the study was to provide an estimation of the frequency of malignant transformation of HCAs and to discuss its clinical implications.

Methods: A systematic literature search was conducted using the following databases: The Cochrane Hepatobiliary Group Controlled Trials Register, The Cochrane Central Register of Controlled Trials (CENTRAL), PubMed, MEDLINE and EMBASE.

Results: 157 relevant series and 17 case reports (a total of 1635 HCAs) were retrieved, reporting an overall frequency of malignant transformation of $4.2 \%$. Only three cases (4.4\%) of malignant alteration were reported in a tumour smaller than $5 \mathrm{~cm}$ in diameter.

Conclusion: Malignant transformation of HCAs into HCCs remains a rare phenomenon with a reported frequency of $4.2 \%$. A better selection of exactly those patients presenting with an HCA with an amplified risk of malignant degeneration is advocated in order to reduce the number of liver resections and thus reducing the operative risk for these predominantly young patients. The Bordeaux adenoma tumour markers are a promising method of identifying these high-risk adenomas. 


\section{INTRODUCTION}

Hepatocellular adenomas (HCAs) are uncommon and essentially benign tumours in the liver that occur predominantly, but not exclusively, in young women taking oral contraceptives (OCs). ${ }^{1,2}$ HCAs are caused by benign proliferation of hepatocytes with high glycogen and fat content but lack normal hepatic architecture. They usually present as a solitary nodule that may reach up to $30 \mathrm{~cm}$ in diameter.

Clinical manifestations range from asymptomatic presentation or abdominal pain localized to the epigastric region or right upper quadrant to a palpable liver mass or even life-threatening haemorrhage in the case of rupture. ${ }^{3,4}$ However, these tumours are most often encountered as an incidental finding during imaging for unrelated pathology.

Although the exact pathogenetic mechanism of the development of HCAs remains unknown, an association between formation of HCAs and the use of OCs or androgen-containing anabolic steroids is assumed. ${ }^{5-7}$ Studies from the past century suggest that long-term use of OCs increases the annual incidence of HCAs from 1 per million to 3 to 4 per $100,000 .^{1,8}$ In addition, OCs and androgen-containing steroid anabolics have also been suggested of increasing the number and size of these adenomas. Conversely, HCAs may shown signs of regression on discontinuance of OC use. ${ }^{9,10}$ HCAs are further reported to be associated with type I and type III glycogen storage disease (GSD) and, furthermore, are more likely to be multifocal or to become malignant in these patients. ${ }^{11}$

The most important complications of HCAs are haemorrhage and malignant transformation into hepatocellular carcinomas (HCCs). Thus, these are the two main reasons for surgical treatment. However, little is known about the true incidence, associated risk factors and the aetiology of malignant alteration of HCAs. The aim of the current systematic review is to provide an estimation of the frequency of this phenomenon by means of a systematic literature search and, moreover, to discuss the clinical implications.

\section{METHODS}

\section{Search strategy}

A search of all literature up to February 2010 was performed independently by two investigators (JHMBS and RJSC) employing all relevant databases including the MEDLINE, PubMed and EMBASE databases, The Cochrane Hepatobiliary Group Controlled Trials Register and The Cochrane Central Register of Controlled Trials (CENTRAL). Keywords were "hepatocellular adenoma", "benign liver tumours", "malignant transformation", and "liver resection". The search was limited to stud- 
ies restricted to humans and articles published from 1970 onwards. This time period was chosen since the number of reports on hepatocellular adenomas began rising in the seventies. All titles and abstracts were screened and relevant articles were selected.

\section{Study selection criteria}

Studies were included if they described a series of patients with HCAs undergoing surgery, embolisation or other (conservative) treatment. Case reports and imaging studies of these benign lesions were also included. Only those studies containing a definite histological diagnosis of the tumours were included. Studies concerning HCAs in patients with GSD were excluded since these patients carry a higher risk of developing these lesions and, moreover, are thought to have an increased risk of malignant degeneration. Furthermore, patients with adenomatosis (more than 10 HCAs) were excluded, as this is considered as a different entity. ${ }^{12,13}$ No further formal quality assessment or selection criteria were employed.

\section{Data extraction}

The reference lists of retrieved articles were reviewed for potentially relevant studies and case reports were also reviewed. When the full text of an article was not available, an Inter Library Loan account was used to retrieve these articles from national libraries. All data of selected articles were screened for duplicate adenoma cases that had already been reported in prior studies. In the case of overlapping series, only the most recent or complete publication was included. The corresponding author of relevant studies identified from the initial search, together with experts in the field, were contacted for any information on unpublished articles and in case of need for clarification.

\section{Outcome measures and statistical analysis}

The main outcome measure was the rate of malignant transformation. In addition, the numbers of (resected) HCAs, the number of females, mean age at presentation, mean diameter of the lesion, $\mathrm{OC}$ use and presence of haemorrhage were all assessed. All reported adenoma cases were listed in a table. All data were presented as mean or median values and percentages. 


\section{RESULTS}

A total of 3935 articles were identified through the electronic searches of PubMed ( $n=120$ ), The Cochrane Hepatobiliary Group Controlled Trials Register and CENTRAL in the Cochrane Library $(n=12)$ and a combined Ovid MEDLINE and EMBASE search ( $n=3803$ ). Through reading titles and abstracts 1196 duplicates as well as 2658 noticeably irrelevant articles and eight articles on GSD or adenomatosis were excluded. Furthermore, four articles were excluded since they contained a series of adenomas that had been previously reported. Altogether, 69 articles were selected for more detailed evaluation. From this analysis, a further 108 studies were included through cross-referencing and three studies were excluded because of previously reported series of adenomas. In total, 174 articles (157 series and 17 case reports) on liver surgery and imaging, including case series of HCA, were retrieved (figure 1).

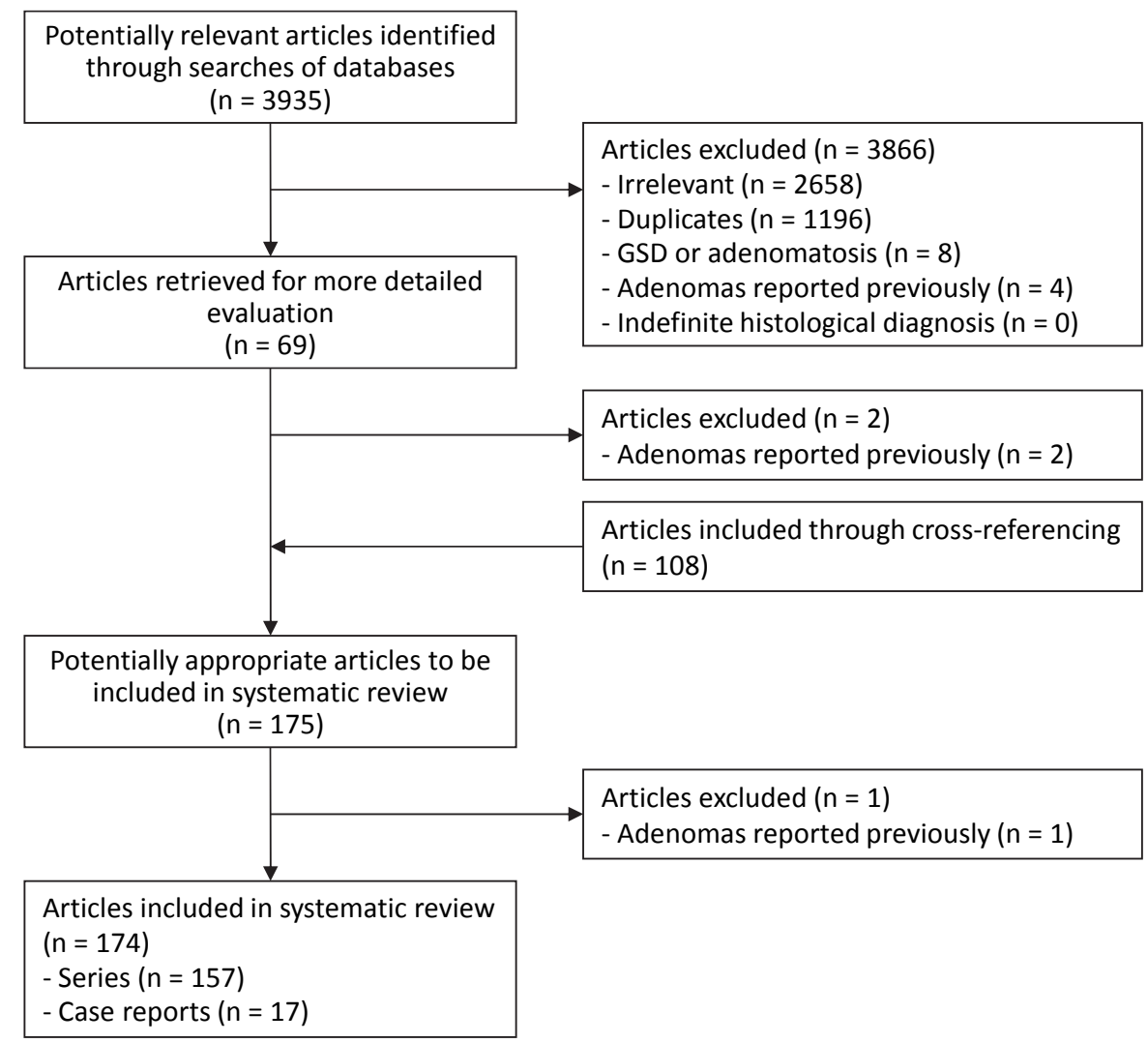

Figure 1. Flowchart literature search. GSD, glycogen storage disease 


\section{Hepatocellular adenoma series in the literature}

The 157 series contained a total of 1617 HCAs worldwide, of which 1445 HCAs $(89 \%)$ were resected (table 1). Thirty-six patients with adenomatosis and 14 patients with GSD were excluded from a total of eight studies. There were 51 series that contained only a single patient with HCA. Most of these reports were published in the seventies of the former century when individual cases of HCA in women using OCs were regularly reported. The three largest series contained $91,{ }^{14} 124^{15}$ and $128^{16}$ HCAs respectively (patients having adenomatosis or GSD not included).

\section{Case reports on malignant transformation of hepatocellular adenomas}

The literature search identified 17 case reports concerning malignant transformation (a total of 19 cases), which are presented in table 2 . The mean age of these patients at the time of surgery was 41 years (range, 19 to 70). Five of these patients (26\%) were men, of whom one had a history of oral prednisolone use and another had a history of anabolic steroid use. ${ }^{17,18}$ Twelve of the 14 women reported to have malignant alteration of an HCA presented with a history of OC usage. The mean time elapsed between commencement of OC therapy and diagnosis of HCA was 14 years. Most cases of malignant transformation of HCAs were seen the time of the diagnosis of HCA. Furthermore, only three cases (16\%) among these 19 cases of malignant degeneration presented with multiple $\mathrm{HCAs}^{19-21}$ and six cases (32\%) were complicated by haemorrhage. ${ }^{21-26}$

Although some authors noted that HCAs may regress on discontinuation of oral contraceptive use, ${ }^{9,10,22}$ three case reports suggested that even after discontinuation of OC use, HCC can still develop irrespective of the occurrence of regression of the $\mathrm{HCA}^{20,22,26}$. Moreover, the reports by Chuang et al. ${ }^{17}$ and Colovic et al. ${ }^{27}$ showed that malignant transformation of HCAs can occur in patients without a history of OC use. 


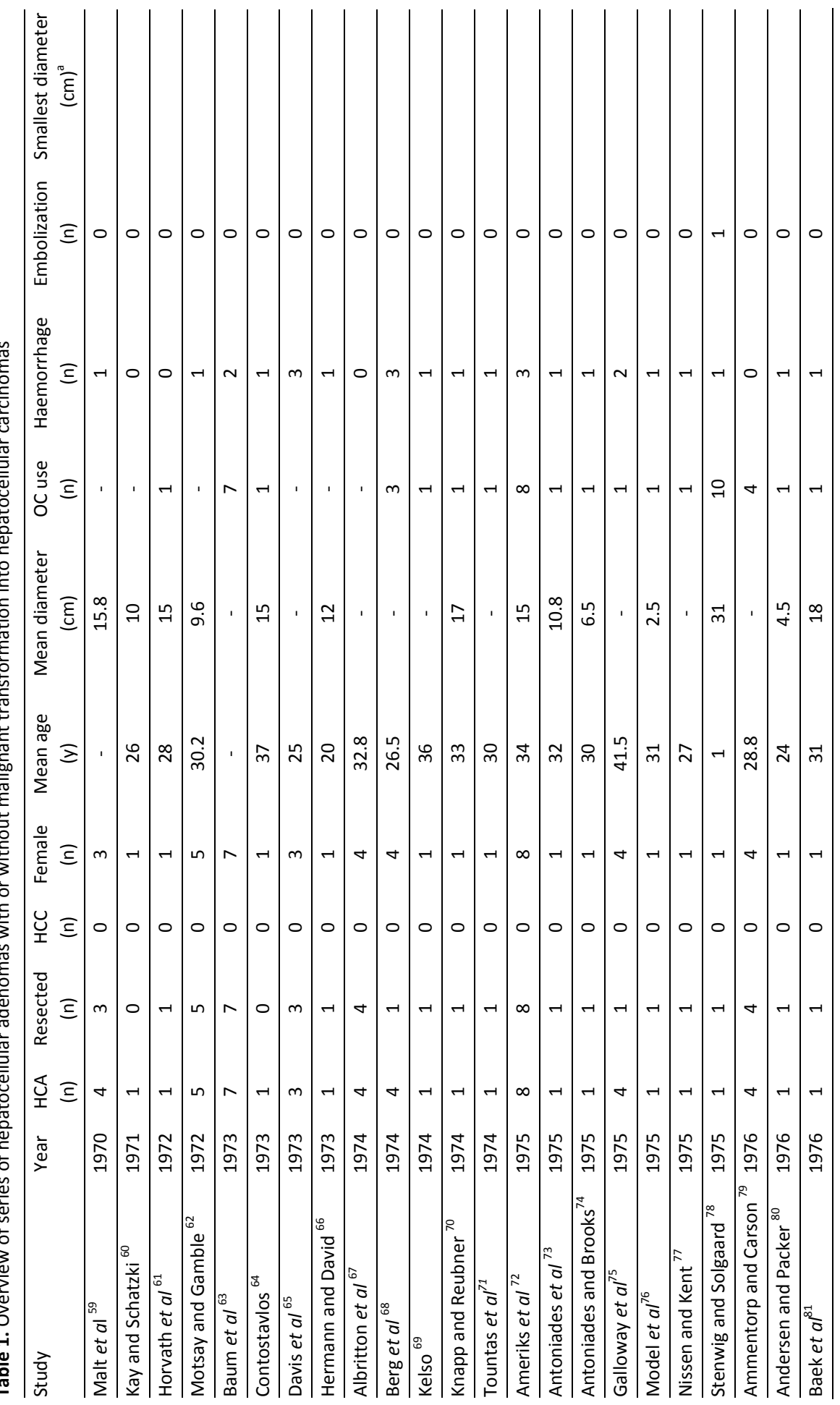




\section{Chapter 8}
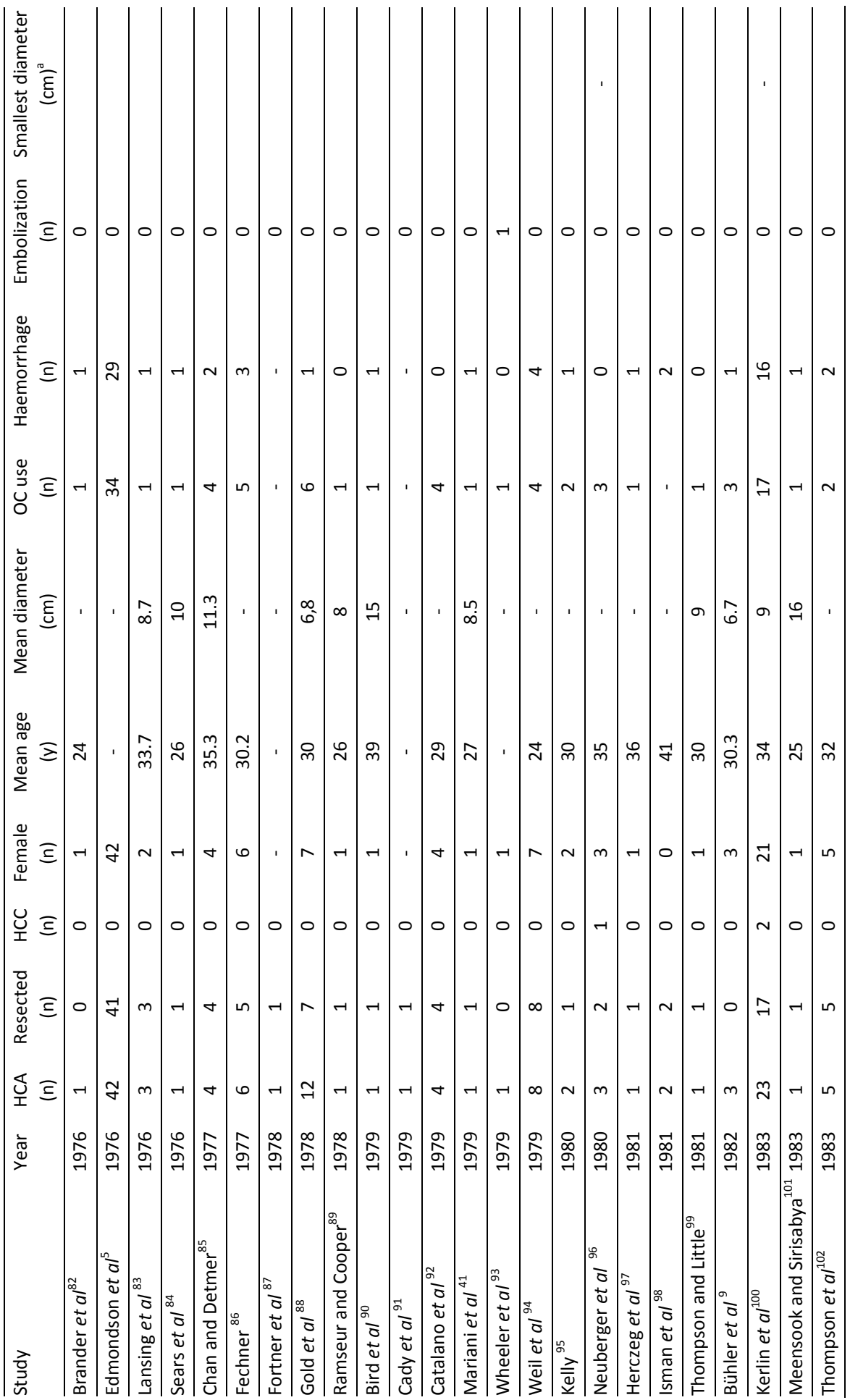


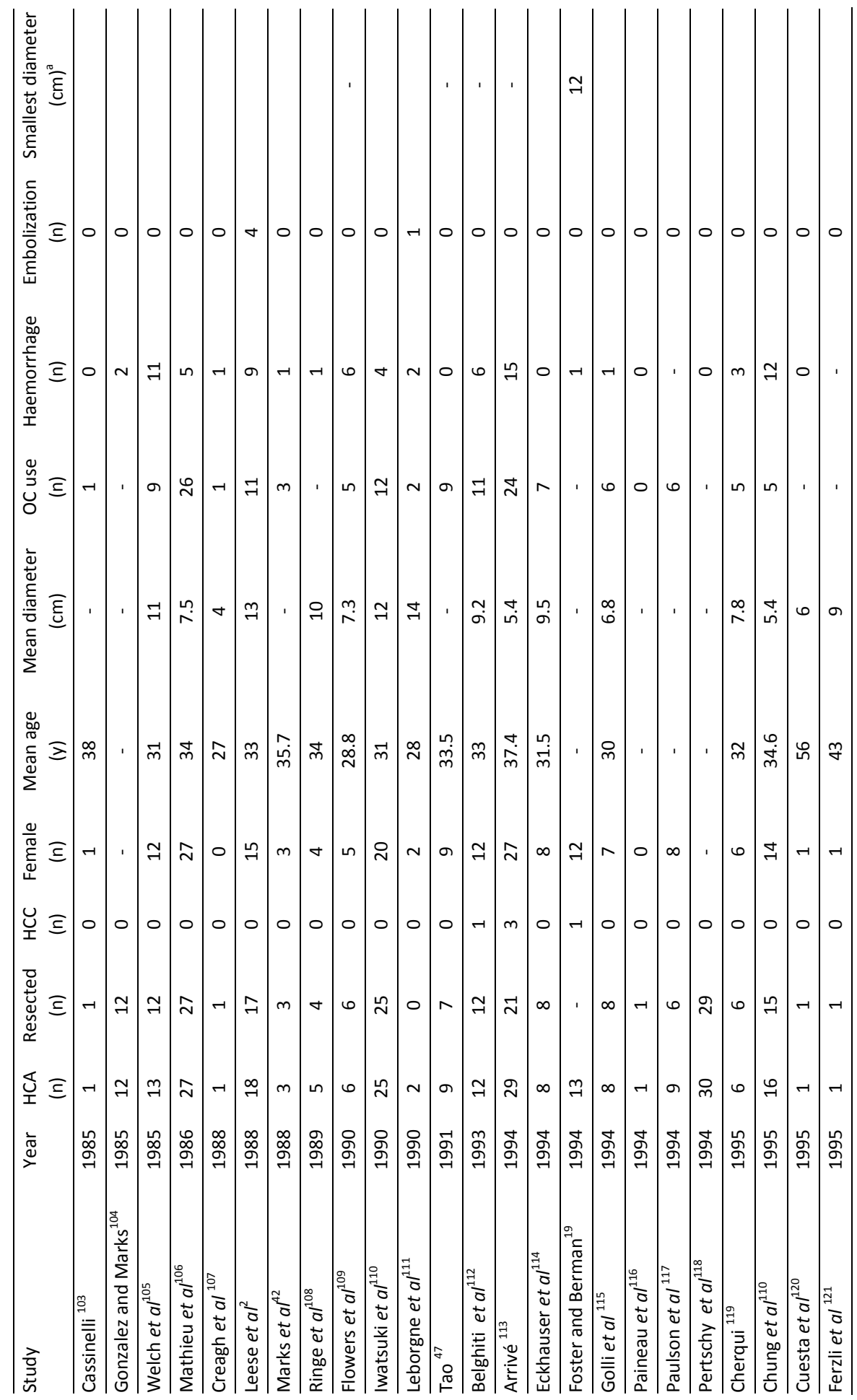




\section{Chapter 8}

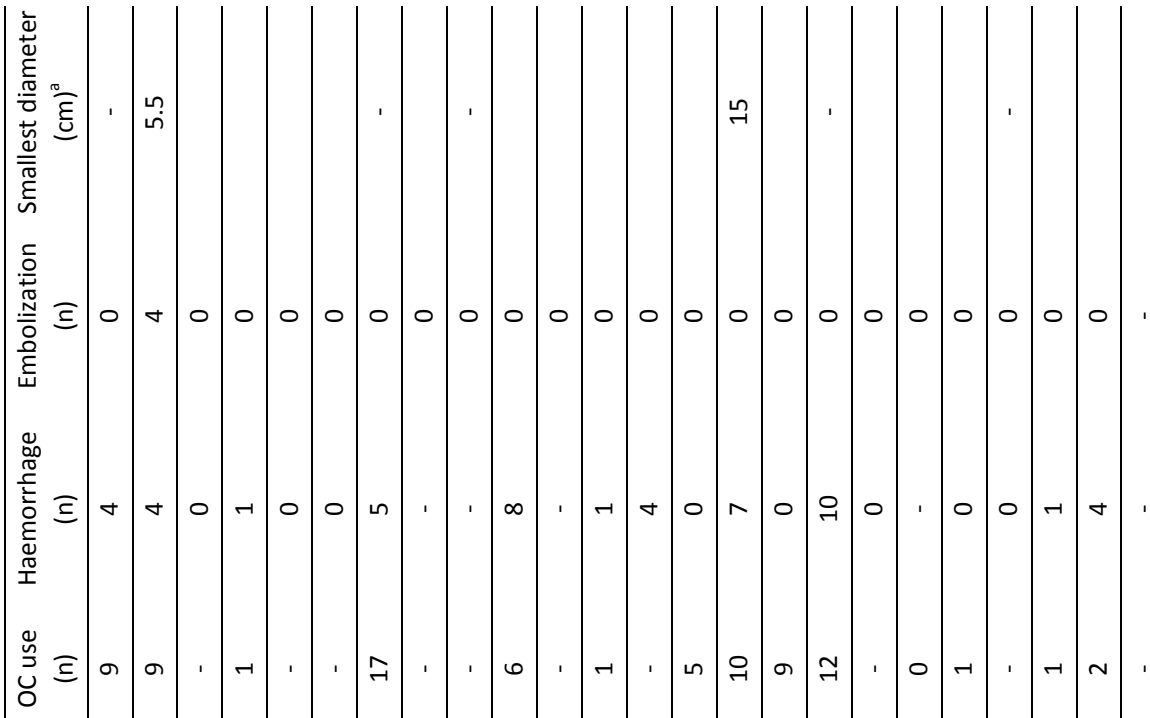
㐫

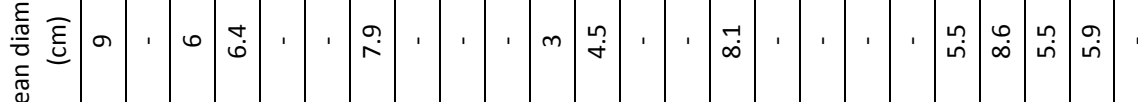
$\stackrel{\sqrt[\pi]{\mathbb{2}}}{\Sigma}$

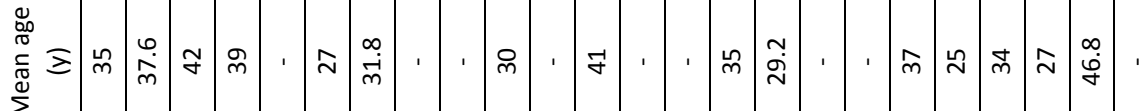

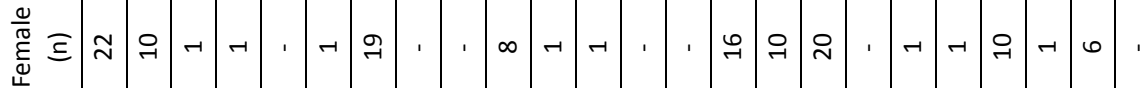

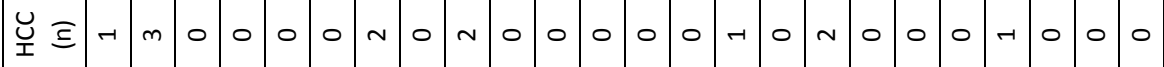
总

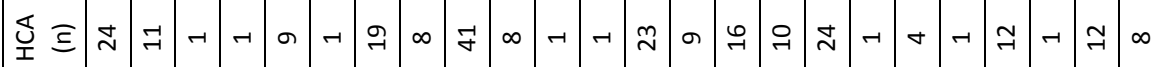
\

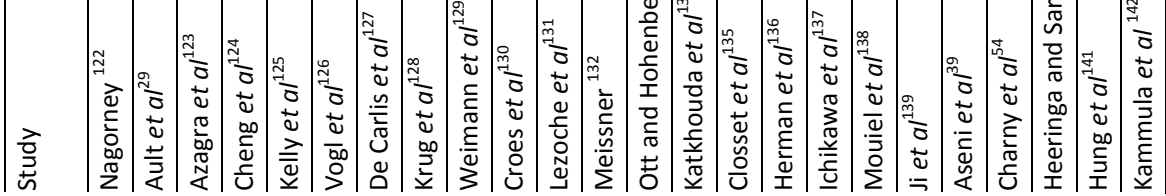




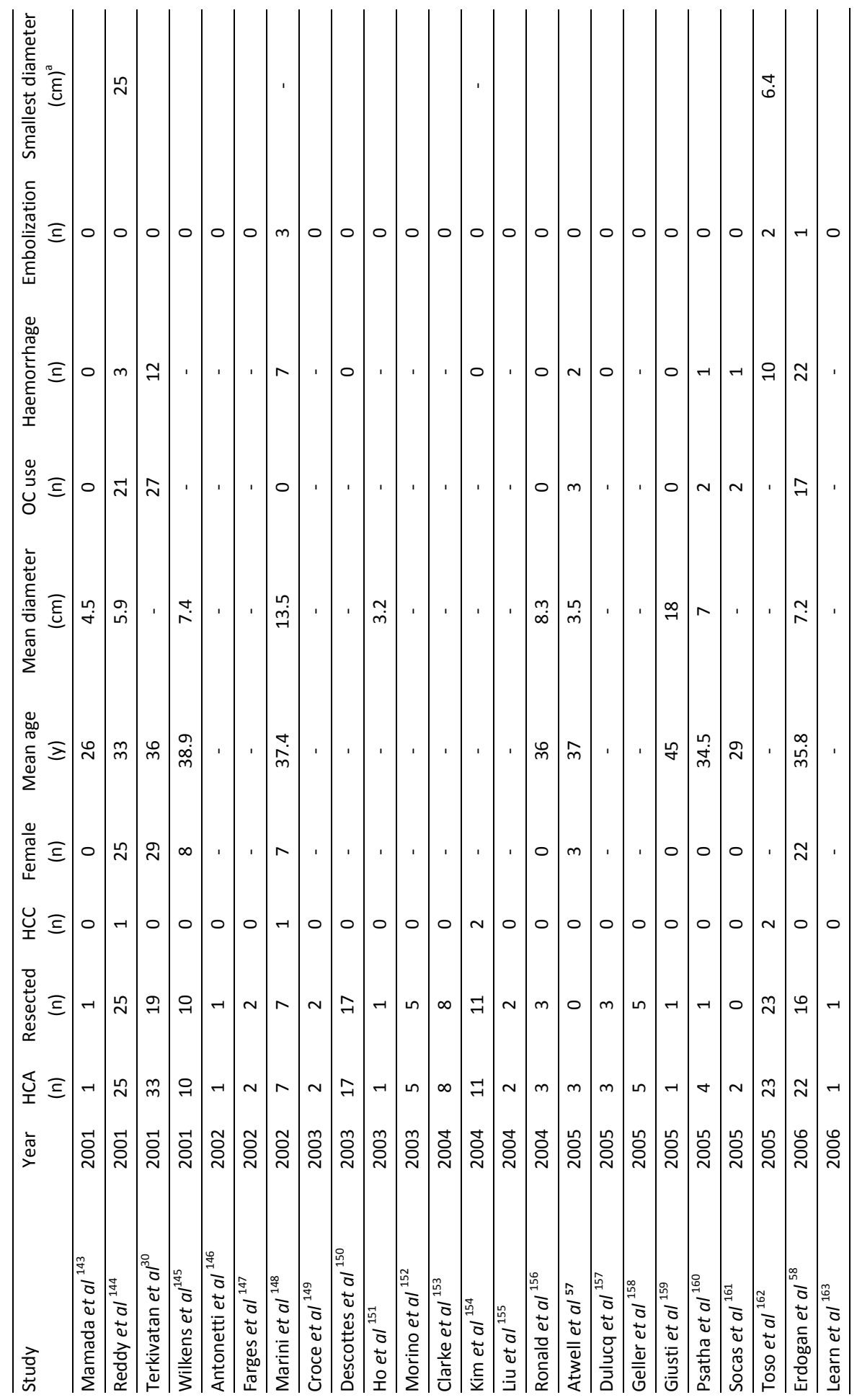




\section{Chapter 8}

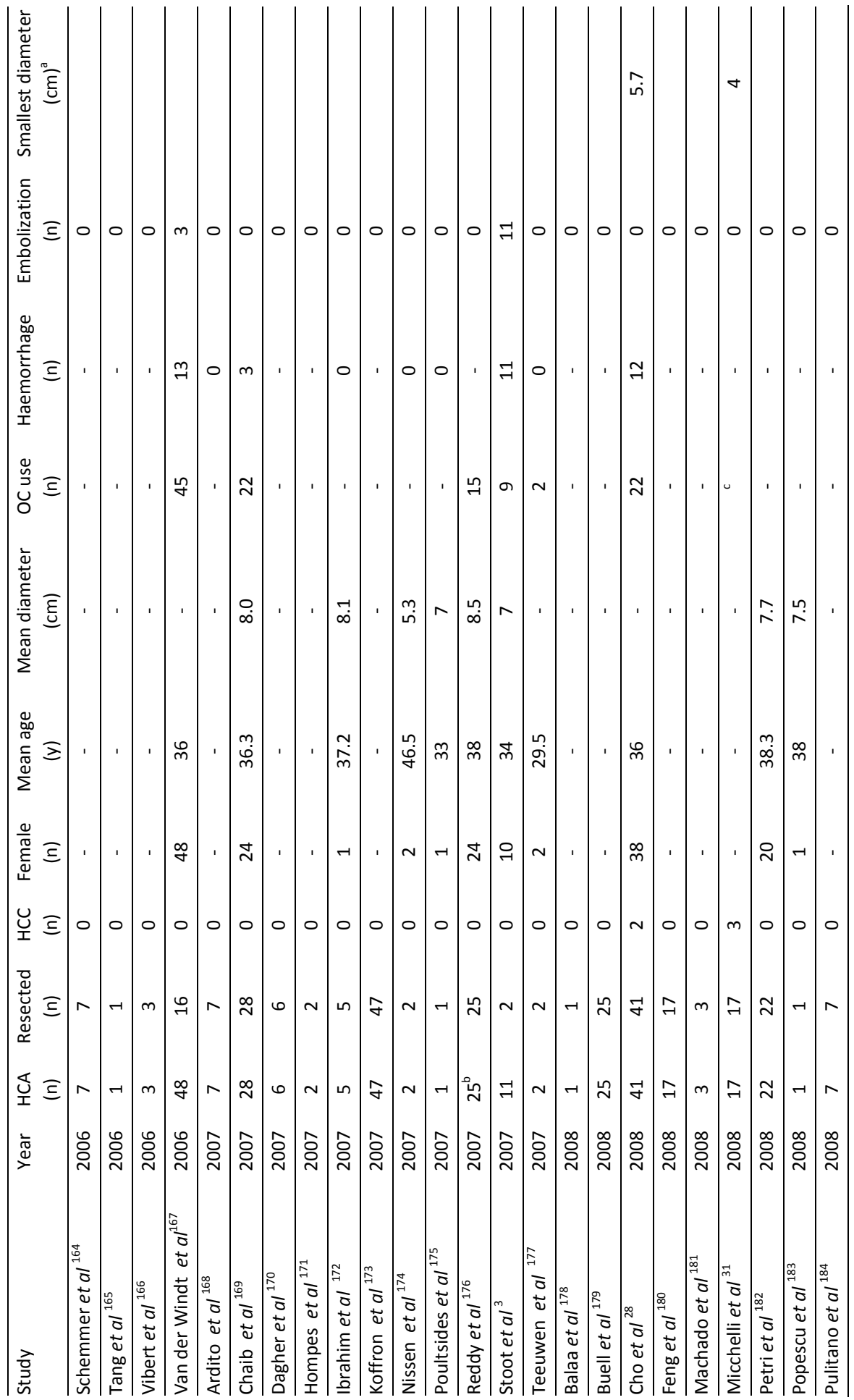




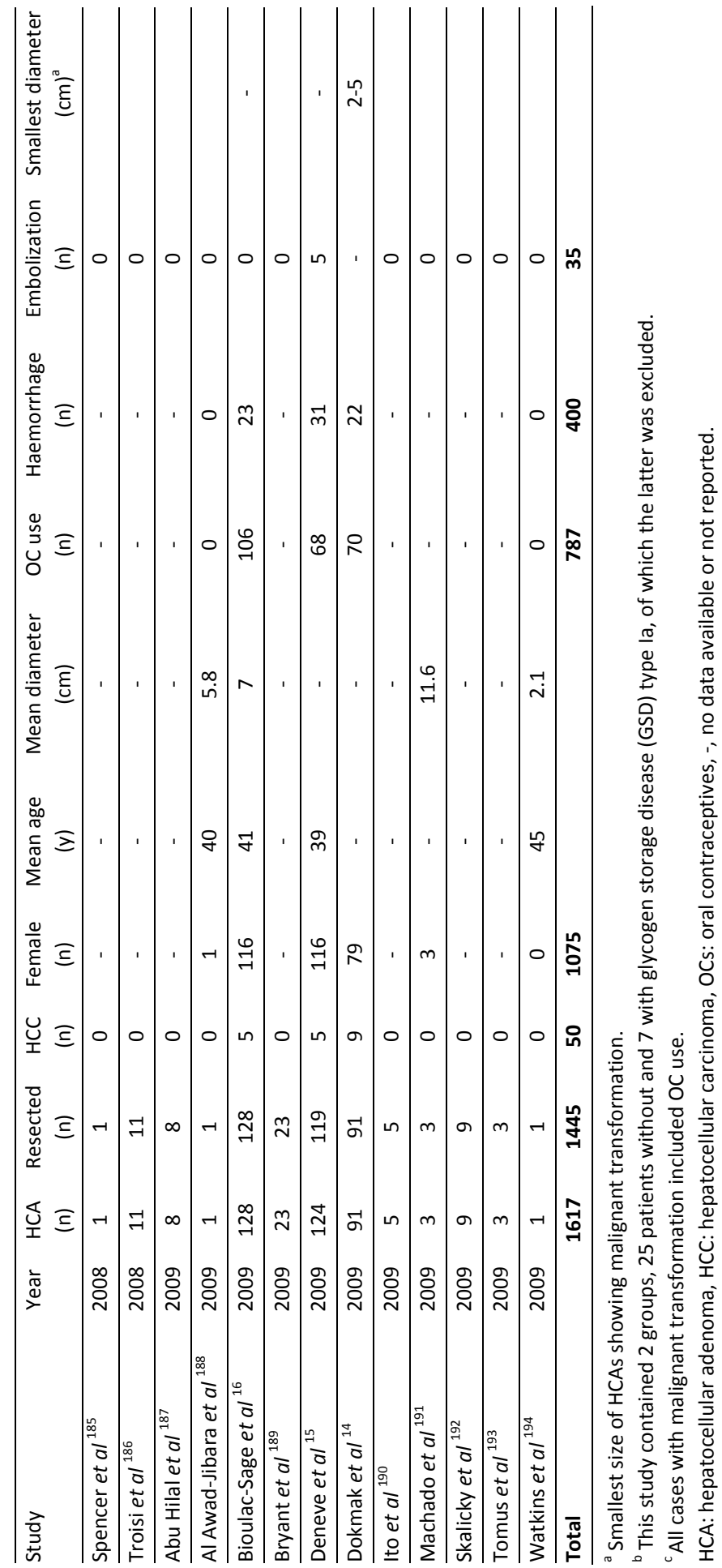




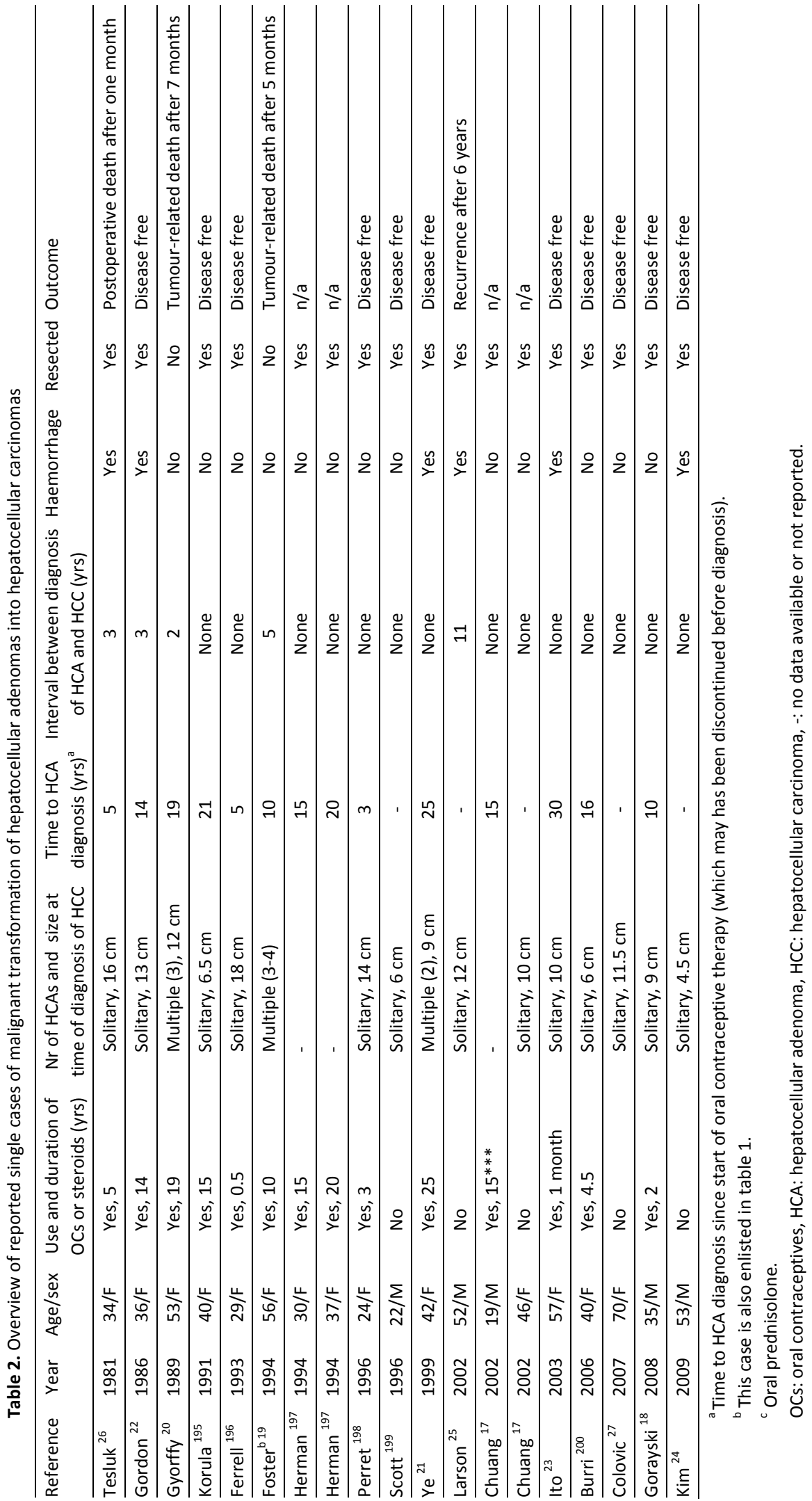




\section{Frequency of malignant transformation of hepatocellular adenomas in the literature}

Out of the 1617 HCAs listed in table 1, 50 tumours (3.1\%) underwent malignant transformation into HCC. By combining these data with the case reports aforementioned (table 2), an estimation of the exact frequency of malignant alteration of HCAs could be made, as the data showed that 68 of a total of 1635 (4.2\%) HCAs underwent malignant transformation. Moreover, of all resected HCAs (1462 in total), $4.5 \%$ contained focal malignancy. Although not the main scope of the current review, haemorrhage was found in 406 of 1635 (25\%) tumours, in keeping with literature data. ${ }^{13,14,28}$

\section{Association between size of HCA and risk of malignant transformation}

The size of HCA has, by current consensus, remained the main decision criterion in determining whether or not resection is indicated, based upon the observation that intratumoral bleeding only seldom takes place in lesions smaller than $5 \mathrm{~cm}$. $2,15,29$ Concordant to this observation, most of the resected adenomas identified in the current search were resected after they reached a minimal size of $5 \mathrm{~cm} .{ }^{4,30} \mathrm{How}$ ever, limited data was hitherto reported concerning the possibility of an association between the size of HCAs and the risk of malignant transformation. Particularly, no specific studies on this subject have been conducted and, moreover, most series in the literature did not report precise measurements of the tumours. Nonetheless, some case reports and series of HCAs with particular diameters were reported. Deneve et $a l^{15}$ analysed 124 patients with an HCA, of which five cases contained signs of malignant alteration. The mean size of these five tumours was $11.6 \mathrm{~cm}$ in largest diameter. No tumour smaller than $8 \mathrm{~cm}$ showed malignant transformation in this study. As shown in table 2, the mean size of solitary HCAs with features of malignant alteration reported in the retrieved case reports was $10.5 \mathrm{~cm}$ (range 4.5 to $18 \mathrm{~cm}$ ). Overall, the smallest size at which malignant transformation was reported in the literature was $4 \mathrm{~cm}$. This malignant alteration occurred in a solitary tumour of a 23-year-old woman who had taken OCs for eight years. ${ }^{31}$ In the series of 91 patients of Dokmak et al, ${ }^{14}$ malignant alteration of either solitary, or multifocal HCAs was seen in nine cases. In this study, only one case of malignant transformation was observed in an adenoma measuring less than $5 \mathrm{~cm}$ in diameter $(2-5 \mathrm{~cm}$, exact size not presented), concerning a male individual with a history of steroid intake. Overall, the current literature search showed that a total of three cases of malignant alteration ( $4.4 \%$ of all HCAs showing this phenomenon) occurred in a tumour measuring less than $5 \mathrm{~cm}$ in diameter. ${ }^{14,24,31}$ 


\section{DISCUSSION}

This systematic review has focussed on the risk of malignant transformation of HCAs into HCCs. The present study shows that malignant alteration is a rare complication of these uncommon benign tumours. By performing a systematic search of studies reporting cases of this benign liver disease over the past 40 years and combining the data on these HCAs, a total of 1568 reported HCAs were found. The overall frequency of malignant transformation reported was $4.2 \%$ among all adenoma cases and $4.5 \%$ among all resected HCAs.

Although earlier series of HCAs had already shown one or two cases, Foster and Berman ${ }^{19}$ were the first to report an estimated risk of malignant transformation, as they found a frequency of $13 \%$. Barthelmes and Tait, ${ }^{13}$ Cho et al ${ }^{28}$ and Micchelli et $a l^{31}$ used a similar approach for determining the incidence of malignant degeneration employed in the current study. However, these three studies identified a remarkably smaller number of case series than included in the current review, and, moreover, the latter study did not include cases of HCAs in which no malignant alteration was found. ${ }^{31}$ Additionally, most case series used in the frequency estimation comprised a limited sample size and reported only on resected adenomas. This could have led to an overestimation of the true risk, apart from the selection bias of reported studies and cases in general. The series of Dokmak et $a l^{14}{ }^{14}$ Deneve et $a l^{15}$ and Bioulac-Sage et $a l^{16}$ seem more robust for estimating the frequency of malignant alteration, as these authors analysed a larger population containing 91, 124 and 128 patients respectively (cases with adenomatosis or GSD not included). Hepatic adenomatosis is regarded as a different entity by many authors concerning presentation and size and number of the adenomas, as well as the different therapeutic options. ${ }^{12,13}$ Equally, several groups have recently reported germ line mutations of hepatocyte nuclear factor $1 \alpha$ inactivation in adenomatosis, and this has been suggested to be associated with maturity-onset diabetes of the young type $3 .^{32-34}$ Also, these mutations may have implications on the risk of malignant degeneration. Therefore, and also to limit heterogeneity, liver adenomatosis was excluded in the present review. As previously mentioned, patients with known GSDs are at a higher risk of developing $\mathrm{HCAs}^{11,35}$ and were therefore not included in the current review.

\section{Risk factors of malignant transformation of hepatocellular adenomas}

Although the risk of malignant transformation seems small (4.2\%), this is a serious complication, which cannot be neglected. As HCAs are difficult to discriminate from HCCs, because of similar imaging characteristics and histopathological features, it is important to identify factors that increase the risk of malignant transformation. Unfortunately, in the current study a true risk analysis based on tumour size was 
difficult to perform as many studies only reported the mean size of the adenomas included. However, as three cases have been reported in which malignant transformation occurred in a tumour measuring less than $5 \mathrm{~cm}$ in diameter, the recommendation to only treat HCAs larger than $5 \mathrm{~cm}$ in diameter in order to prevent malignant transformation is open for debate. ${ }^{14,24,31}$

Upon reviewing the literature, several groups of patients were identified as having an increased risk of malignant alteration of these benign liver tumours. High risk groups reported were those patients with a history of androgen or anabolic steroid intake, ${ }^{18}$ patients of male gender ${ }^{14,36}$ and, as previously stated, patients with GSD. ${ }^{11,37,38}$ Furthermore, as has been reported since the late seventies, intake of OCs could potentially play a role in enlargement of existing HCAs. To date, discontinuation of $O C$ usage is still the advice given to patients that are diagnosed with an $\mathrm{HCA}^{13}$ as several reports showed regression in size of the HCAs following cessation of OC intake. ${ }^{9,39,40}$ However, some case reports suggested that discontinuation of OCs will not abort the risk of malignant transformation. ${ }^{41,42}$ Therefore, even after discontinuation of OC use, long-term follow-up of patients with unresected HCAs remains necessary. Another proposed risk factor for malignant alteration is the presence of dysplasia in HCAs. This characteristic harbours a risk of progression to $\mathrm{HCC}^{43-47}$

Several studies have identified mutations of the $\beta$-catenin gene in HCAs and reported that activated $\beta$-catenin mutations deregulate the $\beta$-catenin pathway. This pathway is part of the more complex Wnt signalling pathway which plays a major role in the proliferation of liver cells. ${ }^{36,48-50}$ These mutations may thus lead to hyperproliferation of liver cells and, consequently, malignancy. The Bordeaux group has performed genotype-phenotype analyses in HCAs and identified four different tumour subtypes with specific characteristics: (1) hepatocyte nuclear factor $1 \alpha$ (HNF1 $\alpha$ ) mutated (30\%-50\%), (2) $\beta$-catenin-activated (10-15\%), (3) inflammatory $(35 \%)$ and $(4)$ unclassified tumours (5\%-10\%). ${ }^{36,51,52}$ Hepatocellular carcinoma associated with adenoma was found in $46 \%$ of $\beta$-catenin-mutated tumours, whereas this has never been observed in inflammatory lesions and rarely found in HNF1 $\alpha$ mutated tumours. This suggests that HCAs with $\beta$-catenin activation carry a higher risk of malignant transformation. Furthermore, the Bordeaux group identified four immunohistochemical markers that characterise each of the four HCA subtypes with high specificity and sensitivity: liver-fatty acid binding protein (L-FABP), glutamine synthetase (GS), nuclear $\beta$-catenin and serum amyloid A (SAA). ${ }^{53}$ They found absence of L-FABP expression to indicate HNF1 $\alpha$ mutation, whereas combined GS overexpression and nuclear $\beta$-catenin staining suggested $\beta$-catenin-activating mutations. Finally, the Bordeaux group noted that SAA staining and overexpression of $\mathrm{C}$-reactive protein (CRP) predicted inflammatory HCA. These markers have proven to be a promising method to identify adenoma patients at risk of developing HCC. 
Table 3 provides an overview of HCA subtypes and their immunohistochemical markers.

Table 3. Types of HCAs and their immunohistochemical markers

\begin{tabular}{llll}
\hline HCA type & Frequency (\%) & Malignant transformation & Markers \\
\hline$\beta$-catenin activated & $10-15$ & Yes & $\beta$-catenin+/GS+ \\
HNF1 $\alpha$ inactivated & $30-50$ & Rarely & LFABP- \\
Inflammatory & 35 & No & SAA+/CRP+ \\
Unclassified & $5-10$ & No & None \\
\hline
\end{tabular}

$\mathrm{CRP}=\mathrm{C}$-reactive protein, $\mathrm{HCA}=$ hepatocellular adenoma, HNF1 $\alpha=$ hepatocyte nuclear factor $1 \alpha, \mathrm{GS}=$ glutamine synthetase, LFABP = liver-fatty acid binding protein, SAA = serum amyloid $A,+=$ positive, - = negative

\section{Future treatment perspectives}

As for future treatment perspectives, more research is needed to investigate the mechanism of malignant degeneration. Only then can this group of predominantly young patients be withheld a potentially unnecessary liver resection, while this surgical treatment has still a reported morbidity and mortality of up to $27 \%$ and $3 \%$ respectively. ${ }^{30,54-56}$ At present, for all patients presenting with one or more HCAs larger than $5 \mathrm{~cm}$, resection is the treatment option of choice in accordance with the current guidelines. Only $4.2 \%$ of HCAs will have actual foci of HCC, and therefore a considerable number of resections will be performed in vain. However, this rate should preferably be seen as an upper limit of the true frequency as publication bias might have occurred. After all, men who have an anabolic steroid-induced adenoma containing foci of malignancy are more likely to be reported in the literature than those adenoma patients without malignant transformation. Nevertheless, over $95 \%$ of all patients presenting with HCAs measuring over $5 \mathrm{~cm}$, will unnecessarily be exposed to a potentially hazardous surgical procedure. By identifying those patients who will derive most benefit from surgery as their HCA harbours an increased risk for malignant alteration, fewer patients will have to undergo this unnecessary surgery. The Bordeaux HCA markers are a promising risk prediction tool. However, biopsy could lead to haemorrhage, sample errors or tumour seeding, but these potential complications are rare in experienced centres. Also, the value of $\beta$-catenin staining needs to be studied more intensively. Recently, interest has turned toward less invasive procedures to treat patients that present with HCAs larger than $5 \mathrm{~cm}$. There is preliminary evidence to suggest that developments in minimally invasive techniques such as (percutaneous) radiofrequency ablation (RFA) or microwave ablation may alter the treatment of HCA. The limited data so far available suggest that these techniques can be performed with low morbidity 
and zero mortality, but additional research is required to explore their exact role in adenoma treatment. ${ }^{57}$

Recently, two groups have reported the therapeutic effects of selective arterial embolisation to stop haemorrhaging from ruptured adenomas ${ }^{.35}$ They also showed that embolising ruptured adenomas prevented growth of these lesions. Subsequently, selective arterial embolisation was utilised in a number of nonhaemorrhaging adenomas. ${ }^{3}$ During follow-up, none of these adenomas grew and the majority even regressed in size. On examining the haemorrhaging and nonhaemorrhaging adenomas separately, a statistically significant decrease in size was noted in both groups. It is this tumour regression, and its probable subsequent reduction of the risk of severe haemorrhaging and malignant transformation, that might propose selective arterial embolisation as a novel treatment for large unruptured HCAs. As no significant complications from this treatment were reported arterial embolisation of HCAs might be the direction for further future research. ${ }^{3,58}$ To the best of our knowledge, no studies have been performed to specifically investigate this treatment.

\section{Conclusions}

The current review shows that malignant transformation of HCAs into HCCs is a rare complication of these uncommon benign tumours. By pooling data of series and case reports, comprising more than 1600 reported HCAs, we found an overall frequency of malignant transformation of $4.2 \%$ for all adenomas, and of $4.5 \%$ for all resected adenomas. A multicentre study with a large registry of HCAs is paramount for estimating the actual risk of malignant transformation. Further research should focus on the underlying mechanisms of malignant transformation of HCAs into HCCs, associated risk factors and the use of new tumour markers. By means of a better selection of precisely which patients with an HCA present with an increased risk of malignant degeneration - and who could therefore derive the greatest benefit from treatment- a reduction in unnecessary liver resections can be achieved. This would reduce the risks associated with surgery in these predominantly young patients.

\section{REFERENCES}

1. Rooks JB, Ory HW, Ishak KG, Strauss LT, Greenspan JR, Hill AP, et al. Epidemiology of hepatocellular adenoma. The role of oral contraceptive use. Jama. 1979;242(7):644-8.

2. Leese $\mathrm{T}$, Farges $\mathrm{O}$, Bismuth $\mathrm{H}$. Liver cell adenomas. A 12-year surgical experience from a specialist hepato-biliary unit. Ann Surg. 1988;208(5):558-64.

3. Stoot JH, van der Linden E, Terpstra OT, Schaapherder AF. Life-saving therapy for haemorrhaging liver adenomas using selective arterial embolization. Br J Surg. 2007; 88(2): 207-209. 


\section{Chapter 8}

4. Terkivatan T, de Wilt JH, de Man RA, van Rijn RR, Tilanus HW, JN IJ. Treatment of ruptured hepatocellular adenoma. Br J Surg. 2001; 94:1249-1253

5. Edmondson HA, Henderson B, Benton B. Liver-cell adenomas associated with use of oral contraceptives. N Engl J Med. 1976; 294(9):470-2.

6. Soe KL, Soe M, Gluud C. Liver pathology associated with the use of anabolic-androgenic steroids. Liver. 1992;12(2):73-9.

7. Wanless IR, Medline A. Role of estrogens as promoters of hepatic neoplasia. Lab Invest. 1982;46(3):313-20.

8. Reddy KR, Schiff ER. Approach to a liver mass. Semin Liver Dis. 1993;13(4):423-35.

9. Buhler H, Pirovino M, Akobiantz A, Altorfer J, Weitzel M, Maranta E, et al. Regression of liver cell adenoma. A follow-up study of three consecutive patients after discontinuation of oral contraceptive use. Gastroenterology. 1982;82(4):775-82.

10. Edmondson HA, Reynolds TB, Henderson B, Benton B. Regression of liver cell adenomas associated with oral contraceptives. Ann Intern Med. 1977;86(2):180-2.

11. Labrune $\mathrm{P}$, Trioche $\mathrm{P}$, Duvaltier I, Chevalier $\mathrm{P}$, Odievre $\mathrm{M}$. Hepatocellular adenomas in glycogen storage disease type I and III: a series of 43 patients and review of the literature. J Pediatr Gastroenterol Nutr. 1997;24(3):276-9.

12. Flejou JF, Barge J, Menu Y, Degott C, Bismuth H, Potet F, et al. Liver adenomatosis. An entity distinct from liver adenoma? Gastroenterology. 1985;89(5):1132-8.

13. Barthelmes L, Tait IS. Liver cell adenoma and liver cell adenomatosis. HPB (Oxford). 2005;7(3):18696.

14. Dokmak S, Paradis V, Vilgrain V, Sauvanet A, Farges O, Valla D, et al. A Single Centre Surgical Experience of 122 Patients with Single and Multiple Hepatocellular Adenomas. Gastroenterology. 2009;137:1698-705

15. Deneve JL, Pawlik TM, Cunningham S, Clary B, Reddy S, Scoggins CR, et al. Liver cell adenoma: a multicentre analysis of risk factors for rupture and malignancy. Ann Surg Oncol. 2009;16(3):640-8.

16. Bioulac-Sage P, Laumonier H, Couchy G, Le Bail B, Sa Cunha A, Rullier A, et al. Hepatocellular adenoma management and phenotypic classification: the Bordeaux experience. Hepatology. 2009;50(2):481-9.

17. Chuang WY, Chen TC, Hsu HL, Lee WC, Jeng LB, Huang SF. Liver cell adenoma with concomitant hepatocellular carcinoma: report of two cases. J Formos Med Assoc. 2002 Nov;101(11):798-802.

18. Gorayski $\mathrm{P}$, Thompson $\mathrm{CH}$, Subhash HS, Thomas AC. Hepatocellular carcinoma associated with recreational anabolic steroid use. Br J Sports Med. 2008;42(1):74-5; discussion 5.

19. Foster JH, Berman MM. The malignant transformation of liver cell adenomas. Arch Surg. 1994;129(7):712-7.

20. Gyorffy EJ, Bredfeldt JE, Black WC. Transformation of hepatic cell adenoma to hepatocellular carcinoma due to oral contraceptive use. Ann Intern Med. 1989;110(6):489-90.

21. Ye MQ, Suriawinata A, Ben Haim M, Parsons R, Schwartz ME. A 42-year-old woman with liver masses and long-term use of oral contraceptives. Semin Liver Dis. 1999;19(3):339-44.

22. Gordon SC, Reddy KR, Livingstone AS, Jeffers LJ, Schiff ER. Resolution of a contraceptive-steroidinduced hepatic adenoma with subsequent evolution into hepatocellular carcinoma. Ann Intern Med. 1986;105(4):547-9.

23. Ito $\mathrm{M}$, Sasaki $\mathrm{M}$, Wen $\mathrm{CY}$, Nakashima $\mathrm{M}$, Ueki $\mathrm{T}$, Ishibashi $\mathrm{H}$, et al. Liver cell adenoma with malignant transformation: a case report. World J Gastroenterol. 2003;9(10):2379-81.

24. Kim DH, Kim SU, Nam DH, Choi YJ, Park SM, Lee CK, et al. A case of hepatocellular carcinoma within hepatocellular adenoma in a non-cirrhotic male. Korean J Intern Med. 2009;24(2):147-52.

25. Larson KA, Weber SM, Fong Y, Blumgart LH. Malignant transformation of hepatic adenoma with recurrence after resection. HPB (Oxford). 2002;4(3):139-43.

26. Tesluk H, Lawrie J. Hepatocellular adenoma. Its transformation to carcinoma in a user of oral contraceptives. Arch Pathol Lab Med. 1981;105(6):296-9. 
27. Colovic R, Grubor N, Micev M, Radak V. Hepatocellular adenoma with malignant alteration. Hepatogastroenterology. 2007;54(74):386-8.

28. Cho SW, Marsh JW, Steel J, Holloway SE, Heckman JT, Ochoa ER, et al. Surgical management of hepatocellular adenoma: take it or leave it? Ann Surg Oncol. 2008 Oct;15(10):2795-803.

29. Ault GT, Wren SM, Ralls PW, Reynolds TB, Stain SC. Selective management of hepatic adenomas. Am Surg. 1996;62(10):825-9.

30. Terkivatan T, de Wilt JH, de Man RA, van Rijn RR, Zondervan PE, Tilanus HW, et al. Indications and long-term outcome of treatment for benign hepatic tumors: a critical appraisal. Arch Surg. 2001;136(9):1033-8.

31. Micchelli ST, Vivekanandan P, Boitnott JK, Pawlik TM, Choti MA, Torbenson M. Malignant transformation of hepatic adenomas. Mod Pathol. 2008;21(4):491-7.

32. Montano-Loza A, Rios-Vaca A, Remes-Troche JM, Meza-Junco J, Trinidad-Hernandez S. Hepatic adenomatosis in an Hispanic patient. A case report and review of the literature. Ann Hepatol. 2002;1(3):136-9.

33. Bacq $Y$, Jacquemin E, Balabaud C, Jeannot E, Scotto B, Branchereau S, et al. Familial liver adenomatosis associated with hepatocyte nuclear factor 1alpha inactivation. Gastroenterology. 2003;125(5):1470-5.

34. Greaves WO, Bhattacharya B. Hepatic adenomatosis. Arch Pathol Lab Med. 2008;132(12):1951-5.

35. Bioulac-Sage $\mathrm{P}$, Laumonier H, Laurent C, Zucman-Rossi J, Balabaud C. Hepatocellular adenoma: what is new in 2008. Hepatol Int. 2008;2(3):316-21.

36. Zucman-Rossi J, Jeannot E, Nhieu JT, Scoazec JY, Guettier C, Rebouissou S, et al. Genotypephenotype correlation in hepatocellular adenoma: new classification and relationship with HCC. Hepatology. 2006;43(3):515-24.

37. Franco LM, Krishnamurthy V, Bali D, Weinstein DA, Arn P, Clary B, et al. Hepatocellular carcinoma in glycogen storage disease type la: a case series. J Inherit Metab Dis. 2005;28(2):153-62.

38. Velazquez I, Alter BP. Androgens and liver tumors: Fanconi's anemia and non-Fanconi's conditions. Am J Hematol. 2004;77(3):257-67.

39. Aseni P, Sansalone CV, Sammartino C, Benedetto FD, Carrafiello G, Giacomoni A, et al. Rapid disappearance of hepatic adenoma after contraceptive withdrawal. J Clin Gastroenterol. 2001;33(3):2346.

40. Kawakatsu M, Vilgrain V, Erlinger S, Nahum H. Disappearance of liver cell adenoma: CT and MR imaging. Abdom Imaging. 1997;22(3):274-6.

41. Mariani AF, Livingstone AS, Pereiras RV, Jr., van Zuiden PE, Schiff ER. Progressive enlargement of an hepatic cell adenoma. Gastroenterology. 1979;77(6):1319-25.

42. Marks WH, Thompson N, Appleman H. Failure of hepatic adenomas (HCA) to regress after discontinuance of oral contraceptives. An association with focal nodular hyperplasia (FNH) and uterine leiomyoma. Ann Surg. 1988;208(2):190-5.

43. Anthony PP, Vogel CL, Barker LF. Liver cell dysplasia: a premalignant condition. J Clin Pathol. 1973;26(3):217-23.

44. Ho JC, Wu PC, Mak TK. Liver cell dysplasia in association with hepatocellular carcinoma, cirrhosis and hepatitis B surface antigen in Hong Kong. Int J Cancer. 1981;28(5):571-4.

45. Lee RG, Tsamandas AC, Demetris AJ. Large cell change (liver cell dysplasia) and hepatocellular carcinoma in cirrhosis: matched case-control study, pathological analysis, and pathogenetic hypothesis. Hepatology. 1997;26(6):1415-22.

46. Su Q, Benner A, Hofmann WJ, Otto G, Pichlmayr R, Bannasch P. Human hepatic preneoplasia: phenotypes and proliferation kinetics of foci and nodules of altered hepatocytes and their relationship to liver cell dysplasia. Virchows Arch. 1997;431(6):391-406.

47. Tao LC. Oral contraceptive-associated liver cell adenoma and hepatocellular carcinoma. Cytomorphology and mechanism of malignant transformation. Cancer. 1991;68(2):341-7. 


\section{Chapter 8}

48. Chen YW, Jeng YM, Yeh SH, Chen PJ. P53 gene and Wnt signaling in benign neoplasms: betacatenin mutations in hepatic adenoma but not in focal nodular hyperplasia. Hepatology. 2002;36:927-35.

49. Nelson WJ, Nusse R. Convergence of Wnt, beta-catenin, and cadherin pathways. Science. 2004;303(5663):1483-7.

50. Thompson MD, Monga SP. WNT/beta-catenin signaling in liver health and disease. Hepatology. 2007;45(5):1298-305.

51. Bioulac-Sage P, Blanc JF, Rebouissou S, Balabaud C, Zucman-Rossi J. Genotype phenotype classification of hepatocellular adenoma. World J Gastroenterol. 2007;13(19):2649-54.

52. Bluteau O, Jeannot E, Bioulac-Sage P, Marques JM, Blanc JF, Bui H, et al. Bi-allelic inactivation of TCF1 in hepatic adenomas. Nat Genet. 2002;32(2):312-5.

53. Bioulac-Sage P, Rebouissou S, Thomas C, Blanc JF, Saric J, Sa Cunha A, et al. Hepatocellular adenoma subtype classification using molecular markers and immunohistochemistry. Hepatology. 2007;46(3):740-8.

54. Charny CK, Jarnagin WR, Schwartz LH, Frommeyer HS, DeMatteo RP, Fong Y, et al. Management of 155 patients with benign liver tumours. Br J Surg. 2001;88(6):808-13.

55. Jarnagin WR, Gonen M, Fong $\mathrm{Y}$, DeMatteo RP, Ben-Porat L, Little S, et al. Improvement in perioperative outcome after hepatic resection: analysis of 1,803 consecutive cases over the past decade. Ann Surg. 2002;236(4):397-406; discussion -7.

56. Poon RT, Fan ST, Lo CM, Liu CL, Lam CM, Yuen WK, et al. Improving perioperative outcome expands the role of hepatectomy in management of benign and malignant hepatobiliary diseases: analysis of 1222 consecutive patients from a prospective database. Ann Surg. 2004;240(4):698-708; discussion -10 .

57. Atwell TD, Brandhagen DJ, Charboneau JW, Nagorney DM, Callstrom MR, Farrell MA. Successful treatment of hepatocellular adenoma with percutaneous radiofrequency ablation. AJR Am J Roentgenol. 2005;184(3):828-31.

58. Erdogan D, Busch OR, van Delden OM, Ten Kate FJ, Gouma DJ, van Gulik TM. Management of spontaneous haemorrhage and rupture of hepatocellular adenomas. A single centre experience. Liver Int. 2006;26(4):433-8.

59. Malt RA, Hershberg RA, Miller WL. Experience with benign tumors of the liver. Surg Gynecol Obstet. 1970;130(2):285-91.

60. Kay S, Schatzki PF. Ultrastructure of a benign liver cell adenoma. Cancer. 1971;28(3):755-62.

61. Horvath E, Kovacs K, Ross RC. Ultrastructural findings in a well-differentiated hepatoma. Digestion. 1972;7(1):74-82.

62. Motsay GJ, Gamble WG. Clinical experience with hepatic adenomas. Surg Gynecol Obstet. 1972;134(3):415-8.

63. Baum JK, Bookstein JJ, Holtz F, Klein EW. Possible association between benign hepatomas and oral contraceptives. Lancet. 1973;2(7835):926-9.

64. Contostavlos DL. Letter: Benign hepatomas and oral contraceptives. Lancet. 1973;2(7839):1200.

65. Davis JB, Schrenken JR, Zimmerman O. Massive hemoperitoneum from rupture of benign hepatocellular adenoma. Surgery. 1973;73(2):181-4.

66. Hermann RE, David TE. Spontaneous rupture of the liver caused by hepatomas. Surgery. 1973;74(5):715-9.

67. Albritton DR, Tompkins RK, Longmire WP, Jr. Hepatic cell adenoma: A report of four cases. Ann Surg. 1974;180(1):14-9.

68. Berg JW, Ketalaar RJ, Rose EF, Vernon RG. Letter: Hepatomas and oral contraceptives. Lancet. 1974;2(7876):349-50.

69. Kelso DR. Letter: Benign hepatomas and oral contraceptives. Lancet. 1974 Feb 23;1(7852):315-6.

70. Knapp WA, Ruebner BH. Letter: Hepatomas and oral contraceptives. Lancet.;1(7851):270-1.

71. Tountas C, Paraskevas G, Deligeorgi H. Letter: Benign hepatoma and oral contraceptives. Lancet. 1974;1(7870):1351-2. 
72. Ameriks JA, Thompson NW, Frey CF, Appelman HD, Walter JF. Hepatic cell adenomas, spontaneous liver rupture, and oral contraceptives. Arch Surg. 1975;110(5):548-57.

73. Antoniades K, Campbell WN, Hecksher RH, Kessler WB, McCarthy GE, Jr. Liver cell adenoma and oral contraceptives. Double tumor development. Jama. 1975;234(6):628-9.

74. Antoniades $\mathrm{K}$, Brooks CE, Jr. Hemoperitoneum from liver cell adenoma in a patient on oral contraceptives. Surgery. 1975;77(1):137-9.

75. Galloway SJ, Casarella WJ, Lattes R, Seamam WB. Minimal deviation hepatoma. A new entity. Am J Roentgenol Radium Ther Nucl Med. 1975;125(1):184-92.

76. Mdel DG, Fox JA, Jones RW. Letter: Multiple hepatic adenomas associated with an oral contraceptive. Lancet. 1975;1(7911):865.

77. Nissen ED, Kent DR. Liver tumors and oral contraceptives. Obstet Gynecol. 1975;46(4):460-7.

78. Stenwig $A E$, Solgaard $T$. Ruptured benign hepatoma associated with an oral contraceptive. A case report. Virchows Arch A Pathol Anat Histol. 1975;367(4):337-43.

79. Ammentorp PA, Carson RP. Hepatocellular adenoma and oral contraceptives. Ohio State Med J. 1976;72(5):283-6.

80. Andersen PH, Packer JT. Hepatic adenoma. Observations after estrogen withdrawal. Arch Surg. 1976;111(8):898-900.

81. Baek S, Sloane CE, Futterman SC. Benign liver cell adenoma associated with use of oral contraceptive agents. Ann Surg. 1976;183(3):239-42.

82. Brander WL, Vosnides G, Ogg CS, West IE. Multiple hepatocellular tumours in a patient treated with oral contraceptives. Virchows Arch A Pathol Anat Histol. 1976;370(1):69-76.

83. Lansing PB, McQuitty JT, Bradburn DM. Benign liver tumors: what is their relationship to oral contraceptives? Am Surg. 1976;42(10):744-60.

84. Sears HF, Smith G, Powell RD. Hepatic adenoma associated with oral contraceptive use: an unusual clinical presentation. Arch Surg. 1976;111(12):1399-403.

85. Chan CK, Detmer DE. Proper management of hepatic adenoma associated with oral contraceptives. Surg Gynecol Obstet. 1977;144(5):703-6.

86. Fechner RE. Benign hepatic lesions and orally administered contraceptives. A report of seven cases and a critical analysis of the literature. Hum Pathol. 1977;8(3):255-68.

87. Fortner JG, Kim DK, Maclean BJ, Barrett MK, Iwatsuki S, Turnbull AD, et al. Major hepatic resection for neoplasia: personal experience in 108 patients. Ann Surg. 1978 Sep;188(3):363-71.

88. Gold JH, Guzman IJ, Rosai J. Benign tumors of the liver. Pathologic examination of 45 cases. Am J Clin Pathol. 1978 Jul;70(1):6-17.

89. Ramseur WL, Cooper MR. Asymptomatic liver cell adenomas. Another case of resolution after discontinuation of oral contraceptive use. Jama. 1978 Apr 21;239(16):1647-8.

90. Bird D, Vowles K, Anthony PP. Spontaneous rupture of a liver cell adenoma after long term methyltestosterone: report of a case successfully treated by emergency right hepatic lobectomy. $\mathrm{Br} \mathrm{J}$ Surg. 1979;66(3):212-3.

91. Cady B, Bonneval M, Fender HR, Jr. Elective hepatic resection. Am J Surg. 1979;137(4):514-21.

92. Catalano PW, Martin EW, Jr., Ellison C, Carey LC. Reasonable surgical treatment for tumors of the liver associated with the use of oral contraceptives. Surg Gynecol Obstet. 1979;148(5):759-63.

93. Wheeler PG, Melia W, Dubbins P, Jones B, Nunnerley H, Johnson P, et al. Non-operative arterial embolisation in primary liver tumours. Br Med J. 1979;2(6184):242-4.

94. Weil R, 3rd, Koep L, Starzl TE. Liver resection for hepatic adenoma. Arch Surg. 1979;114(2):178-80.

95. Kelly TR. Benign liver tumors: presenting profiles and treatment. Am Surg. 1980;46(7):398-402.

96. Neuberger J, Portmann B, Nunnerley HB, Laws JW, Davis M, Williams R. Oral-contraceptiveassociated liver tumours: occurrence of malignancy and difficulties in diagnosis. Lancet.;1(8163):273-6.

97. Herczeg B, Magyar E, Petroczy G, Kovacs I, Ling L, Csitary F. [Oral contraceptives and liver tumors]. Orv Hetil. 1981;122(31):1879-83. 


\section{Chapter 8}

98. Isman H, Bourgeon R, Rampal P, Koch G, Mattei S. [Benign solid hepatic tumors: report on six cases. Diagnostic and therapeutic problems (author's transl)]. Chirurgie. 1981;107(1):44-52.

99. Thompson JF, Little JM. Liver resection for neoplasm. Aust N Z J Surg. 1981;51(3):274-9.

100. Kerlin P, Davis GL, McGill DB, Weiland LH, Adson MA, Sheedy PF, 2nd. Hepatic adenoma and focal nodular hyperplasia: clinical, pathologic, and radiologic features. Gastroenterology. 1983;84(5 Pt 1):994-1002.

101. Meensook C, Sirisabya N. Benign hepatic tumor and oral contraceptives. J Med Assoc Thai. 1983;66(5):299-302.

102. Thompson HH, Tompkins RK, Longmire WP, Jr. Major hepatic resection. A 25-year experience. Ann Surg. 1983;197(4):375-88.

103. Cassinelli GB. [Hepatic adenoma and oral contraceptives: personal case]. Ann Ital Chir. 1985;57(2):101-7.

104. Gonzalez F, Marks C. Hepatic tumors and oral contraceptives: surgical management. J Surg Oncol. $1985 \mathrm{Jul} ; 29(3): 193-7$.

105. Welch TJ, Sheedy PF, 2nd, Johnson CM, Stephens DH, Charboneau JW, Brown ML, et al. Focal nodular hyperplasia and hepatic adenoma: comparison of angiography, CT, US, and scintigraphy. Radiology. 1985;156(3):593-5.

106. Mathieu D, Bruneton JN, Drouillard J, Pointreau CC, Vasile N. Hepatic adenomas and focal nodular hyperplasia: dynamic CT study. Radiology. 1986;160(1):53-8.

107. Creagh TM, Rubin A, Evans DJ. Hepatic tumours induced by anabolic steroids in an athlete. J Clin Pathol. 1988;41(4):441-3.

108. Ringe B, Mauz S, Barg-Hock H, Kotzerke J, Pichlmayr R. [Surgery of benign liver tumors]. Langenbecks Arch Chir Suppl II Verh Dtsch Ges Chir. 1989:287-91.

109. Flowers BF, McBurney RP, Vera SR. Ruptured hepatic adenoma. A spectrum of presentation and treatment. Am Surg. 1990;56(6):380-3.

110. Iwatsuki S, Todo S, Starzl TE. Excisional therapy for benign hepatic lesions. Surg Gynecol Obstet. 1990;171(3):240-6.

111. Leborgne J, Lehur PA, Horeau JM, Dupas B, Bourcheix LM, Petiot JM, et al. [Therapeutic problems caused by rupture of large hepatic adenoma with central location. Apropos of 3 cases]. Chirurgie. 1990;116(4-5):454-60.

112. Belghiti J, Pateron D, Panis Y, Vilgrain V, Flejou JF, Benhamou JP, et al. Resection of presumed benign liver tumours. Br J Surg. 1993;80(3):380-3.

113. Arrive L, Flejou JF, Vilgrain V, Belghiti J, Najmark D, Zins M, et al. Hepatic adenoma: MR findings in 51 pathologically proved lesions. Radiology. 1994;193(2):507-12.

114. Eckhauser FE, Knol JA, Raper SE, Thompson NW. Enucleation combined with hepatic vascular exclusion is a safe and effective alternative to hepatic resection for liver cell adenoma. Am Surg. 1994;60(7):466-71; discussion 72.

115. Golli M, Van Nhieu JT, Mathieu D, Zafrani ES, Cherqui D, Dhumeaux D, et al. Hepatocellular adenoma: color Doppler US and pathologic correlations. Radiology. 1994;190(3):741-4.

116. Paineau J, Hamy A, Visset J. [Our surgical experience in the resection of benign hepatic tumors. Apropos of 31 cases]. J Chir (Paris). 1994;131(11):461-5.

117. Paulson EK, McClellan JS, Washington K, Spritzer CE, Meyers WC, Baker ME. Hepatic adenoma: MR characteristics and correlation with pathologic findings. AJR Am J Roentgenol. 1994;163(1):113-6.

118. Pertschy J, Ruckert JC, Manger T. [Diagnosis and surgical therapy of benign liver tumors]. Zentralbl Chir. 1994;119(7):495-500.

119. Cherqui D, Rahmouni A, Charlotte F, Boulahdour H, Metreau JM, Meignan M, et al. Management of focal nodular hyperplasia and hepatocellular adenoma in young women: a series of 41 patients with clinical, radiological, and pathological correlations. Hepatology. 1995;22(6):1674-81.

120. Cuesta MA, Meijer S, Paul MA, de Brauw LM. Limited laparoscopic liver resection of benign tumors guided by laparoscopic ultrasonography: report of two cases. Surg Laparosc Endosc. 1995;5(5):396401. 
121. Ferzli G, David A, Kiel T. Laparoscopic resection of a large hepatic tumor. Surg Endosc. 1995;9(6):733-5.

122. Nagorney DM. Benign hepatic tumors: focal nodular hyperplasia and hepatocellular adenoma. World J Surg. 1995;19(1):13-8.

123. Azagra JS, Goergen M, Gilbart E, Jacobs D. Laparoscopic anatomical (hepatic) left lateral segmentectomy-technical aspects. Surg Endosc. 1996;10(7):758-61.

124. Cheng PN, Shin JS, Lin XZ. Hepatic adenoma: an observation from asymptomatic stage to rupture. Hepatogastroenterology. 1996;43(7):245-8.

125. Kelly D, Emre S, Guy SR, Sheiner PA, Miller CM, Schwartz ME. Resection of benign hepatic lesions with selective use of total vascular isolation. J Am Coll Surg. 1996;183(2):113-6.

126. VogI TJ, Hammerstingl R, Schwarz W, Mack MG, Muller PK, Pegios W, et al. Superparamagnetic iron oxide--enhanced versus gadolinium-enhanced MR imaging for differential diagnosis of focal liver lesions. Radiology. 1996 Mar;198(3):881-7.

127. De Carlis L, Pirotta V, Rondinara GF, Sansalone CV, Colella G, Maione G, et al. Hepatic adenoma and focal nodular hyperplasia: diagnosis and criteria for treatment. Liver Transpl Surg. 1997 Mar;3(2):160-5.

128. Krug B, Zieren HU, Jung G, Hemme A, Heindel W, Krings F. Late results after resection of benign hepatic tumors: clinical and radiological findings. Eur Radiol. 1997;7(3):327-32.

129. Weimann A, Ringe B, Klempnauer J, Lamesch P, Gratz KF, Prokop M, et al. Benign liver tumors: differential diagnosis and indications for surgery. World J Surg. 1997;21(9):983-90; discussion 90-1.

130. Croes EA, van Gulik TM, Bosma A, de Wit LT, Gouma DJ. [Treatment of 8 patients with an acute hemorrhage in a hepatocellular adenoma at the Academic Medical Centre, Amsterdam]. Ned Tijdschr Geneeskd. 1998;142(45):2463-8.

131. Lezoche E, Paganini AM, Feliciotti F, Guerrieri M, Lugnani F, Tamburini A. Ultrasound-guided laparoscopic cryoablation of hepatic tumors: preliminary report. World J Surg. 1998;22(8):829-35; discussion 35-6.

132. Meissner K. Hemorrhage caused by ruptured liver cell adenoma following long-term oral contraceptives: a case report. Hepatogastroenterology. 1998;45(19):224-5.

133. Ott R, Hohenberger W. [Focal nodular hyperplasia and liver cell adenoma: operation or observation?]. Zentralbl Chir. 1998;123(2):145-53.

134. Katkhouda N, Hurwitz M, Gugenheim J, Mavor E, Mason RJ, Waldrep DJ, et al. Laparoscopic management of benign solid and cystic lesions of the liver. Ann Surg. 1999;229(4):460-6.

135. Closset J, Veys I, Peny MO, Braude P, Van Gansbeke D, Lambilliotte JP, et al. Retrospective analysis of 29 patients surgically treated for hepatocellular adenoma or focal nodular hyperplasia. Hepatogastroenterology. 2000;47(35):1382-4.

136. Herman P, Pugliese V, Machado MA, Montagnini AL, Salem MZ, Bacchella T, et al. Hepatic adenoma and focal nodular hyperplasia: differential diagnosis and treatment. World J Surg. 2000;24(3):372-6.

137. Ichikawa T, Federle MP, Grazioli L, Nalesnik M. Hepatocellular adenoma: multiphasic CT and histopathologic findings in 25 patients. Radiology. 2000;214(3):861-8.

138. Mouiel J, Katkhouda N, Gugenheim J, Fabiani P. Possibilities of laparoscopic liver resection. J Hepatobiliary Pancreat Surg. 2000;7(1):1-8.

139. Ji Y, Zhu X, Sun H, Tan Y, Ma Z, Ye Q, et al. Hepatocellular adenoma and focal nodular hyperplasia: a series of 24 patients with clinicopathological and radiological correlation. Chin Med J (Engl). 2000;113(9):852-7.

140. Heeringa B, Sardi A. Bleeding hepatic adenoma: expectant treatment to limit the extent of liver resection. Am Surg. 2001;67(10):927-9.

141. Hung $\mathrm{CH}$, Changchien CS, Lu SN, Eng HL, Wang JH, Lee CM, et al. Sonographic features of hepatic adenomas with pathologic correlation. Abdom Imaging. 2001;26(5):500-6.

142. Kammula US, Buell JF, Labow DM, Rosen S, Millis JM, Posner MC. Surgical management of benign tumors of the liver. Int J Gastrointest Cancer. 2001;30(3):141-6. 


\section{Chapter 8}

143. Mamada Y, Onda M, Tajiri T, Akimaru K, Yoshida H, Taniai N, et al. Liver cell adenoma in a 26-yearold man. J Nippon Med Sch. 2001;68(6):516-9.

144. Reddy KR, Kligerman S, Levi J, Livingstone A, Molina E, Franceschi D, et al. Benign and solid tumors of the liver: relationship to sex, age, size of tumors, and outcome. Am Surg. 2001;67(2):173-8.

145. Wilkens L, Bredt M, Flemming P, Becker T, Klempnauer J, Kreipe HH. Differentiation of liver cell adenomas from well-differentiated hepatocellular carcinomas by comparative genomic hybridization. J Pathol. 2001;193(4):476-82.

146. Antonetti MC, Killelea B, Orlando R, 3rd. Hand-assisted laparoscopic liver surgery. Arch Surg. 2002;137(4):407-11; discussion 12.

147. Farges $O$, Jagot $P$, Kirstetter P, Marty J, Belghiti J. Prospective assessment of the safety and benefit of laparoscopic liver resections. J Hepatobiliary Pancreat Surg. 2002;9(2):242-8.

148. Marini P, Vilgrain V, Belghiti J. Management of spontaneous rupture of liver tumours. Dig Surg. 2002;19(2):109-13.

149. Croce E, Olmi S, Bertolini A, Erba L, Magnone S. Laparoscopic liver resection with radiofrequency. Hepatogastroenterology. 2003;50(54):2088-92.

150. Descottes B, Glineur D, Lachachi F, Valleix D, Paineau J, Hamy A, et al. Laparoscopic liver resection of benign liver tumors. Surg Endosc. 2003;17(1):23-30.

151. Ho CL, Yu SC, Yeung DW. 11C-acetate PET imaging in hepatocellular carcinoma and other liver masses. J Nucl Med. 2003;44(2):213-21.

152. Morino M, Morra I, Rosso E, Miglietta C, Garrone C. Laparoscopic vs open hepatic resection: a comparative study. Surg Endosc. 2003;17(12):1914-8.

153. Clarke D, Currie E, Madhavan K, Parks R, Garden O. Hepatic resection for benign non-cystic liver lesions. HPB (Oxford). 2004;6(2):115-9.

154. Kim J, Ahmad SA, Lowy AM, Buell JF, Pennington LJ, Moulton JS, et al. An algorithm for the accurate identification of benign liver lesions. Am J Surg. 2004;187(2):274-9.

155. Liu CL, Fan ST, Lo CM, Chan SC, Tso WK, Ng IO, et al. Hepatic resection for incidentaloma. J Gastrointest Surg. 2004;8(7):785-93.

156. Ronald M, Woodfield J, McCall J, Koea J. Hepatic adenomas in male patients. HPB (Oxford). 2004;6(1):25-7.

157. Dulucq JL, Wintringer $P$, Stabilini C, Berticelli J, Mahajna A. Laparoscopic liver resections: a single centre experience. Surg Endosc. 2005;19(7):886-91.

158. Geller DA, Tsung A, Maheshwari V, Rutstein LA, Fung JJ, Wallis Marsh J. Hepatic resection in 170 patients using saline-cooled radiofrequency coagulation. HPB (Oxford). 2005;7(3):208-13.

159. Giusti S, Donati F, Paolicchi A, Bartolozzi C. Hepatocellular adenoma: imaging findings and pathological correlation. Dig Liver Dis. 2005;37(3):200-5.

160. Psatha EA, Semelka RC, Armao D, Woosley JT, Firat Z, Schneider G. Hepatocellular adenomas in men: MRI findings in four patients. J Magn Reson Imaging. 2005;22(2):258-64.

161. Socas L, Zumbado M, Perez-Luzardo O, Ramos A, Perez C, Hernandez JR, et al. Hepatocellular adenomas associated with anabolic androgenic steroid abuse in bodybuilders: a report of two cases and a review of the literature. Br J Sports Med. 2005;39(5):e27.

162. Toso C, Majno P, Andres A, Rubbia-Brandt L, Berney T, Buhler L, et al. Management of hepatocellular adenoma: solitary-uncomplicated, multiple and ruptured tumors. World J Gastroenterol. 2005;11(36):5691-5.

163. Learn PA, Bowers SP, Watkins KT. Laparoscopic hepatic resection using saline-enhanced electrocautery permits short hospital stays. J Gastrointest Surg. 2006;10(3):422-7.

164. Schemmer P, Friess H, Hinz U, Mehrabi A, Kraus TW, Z'Graggen K, et al. Stapler hepatectomy is a safe dissection technique: analysis of 300 patients. World J Surg. 2006;30(3):419-30.

165. Tang CN, Tsui KK, Ha JP, Yang GP, Li MK. A single-centre experience of 40 laparoscopic liver resections. Hong Kong Med J. 2006;12(6):419-25.

166. Vibert E, Perniceni T, Levard H, Denet C, Shahri NK, Gayet B. Laparoscopic liver resection. Br J Surg. 2006;93(1):67-72. 
167. van der Windt DJ, Kok NF, Hussain SM, Zondervan PE, Alwayn IP, de Man RA, et al. Case-orientated approach to the management of hepatocellular adenoma. Br J Surg. 2006;93(12):1495-502.

168. Ardito F, Tayar C, Laurent A, Karoui M, Loriau J, Cherqui D. Laparoscopic liver resection for benign disease. Arch Surg. 2007;142(12):1188-93; discussion 93.

169. Chaib E, Gama-Rodrigues J, Ribeiro MA, Jr., Herman P, Saad WA. Hepatic adenoma. Timing for surgery. Hepatogastroenterology. 2007;54(77):1382-7.

170. Dagher I, Proske JM, Carloni A, Richa H, Tranchart H, Franco D. Laparoscopic liver resection: results for 70 patients. Surg Endosc. 2007;21(4):619-24.

171. Hompes D, Aerts R, Penninckx F, Topal B. Laparoscopic liver resection using radiofrequency coagulation. Surg Endosc. 2007;21(2):175-80.

172. Ibrahim S, Chen CL, Wang SH, Lin CC, Yang CH, Yong CC, et al. Liver resection for benign liver tumors: indications and outcome. Am J Surg. 2007;193(1):5-9.

173. Koffron AJ, Auffenberg G, Kung R, Abecassis M. Evaluation of 300 minimally invasive liver resections at a single institution: less is more. Ann Surg. 2007;246(3):385-92; discussion 92-4.

174. Nissen NN, Grewal N, Lee J, Nawabi A, Korman J. Completely laparoscopic nonanatomic hepatic resection using saline-cooled cautery and hydrodissection. Am Surg. 2007;73(10):987-90.

175. Poultsides G, Brown M, Orlando R, 3rd. Hand-assisted laparoscopic management of liver tumors. Surg Endosc. 2007;21(8):1275-9.

176. Reddy SK, Kishnani PS, Sullivan JA, Koeberl DD, Desai DM, Skinner MA, et al. Resection of hepatocellular adenoma in patients with glycogen storage disease type la. J Hepatol. 2007;47(5):658-63.

177. Teeuwen PH, Ruers TJ, Wobbes T. [Hepatocellular adenoma, a tumour particularly seen in mostly young women]. Ned Tijdschr Geneeskd. 2007;151(24):1321-4.

178. Balaa FK, Gamblin TC, Tsung A, Marsh JW, Geller DA. Right hepatic lobectomy using the staple technique in 101 patients. J Gastrointest Surg. 2008;12(2):338-43.

179. Buell JF, Thomas MT, Rudich S, Marvin M, Nagubandi R, Ravindra KV, et al. Experience with more than 500 minimally invasive hepatic procedures. Ann Surg. 2008;248(3):475-86.

180. Feng ZQ, Huang ZQ, Xu LN, Liu R, Zhang $A Q$, Huang $X Q$, et al. Liver resection for benign hepatic lesions: a retrospective analysis of 827 consecutive cases. World J Gastroenterol. 2008;14(47):7247-51.

181. Machado MA, Makdissi FF, Galvao FH, Machado MC. Intrahepatic Glissonian approach for laparoscopic right segmental liver resections. Am J Surg. 2008;196(4):e38-42.

182. Petri A, Hohn J, Kokai EL, Savanya GK, Lazar G. Surgery of benign liver tumors: indications for treatment: twenty years' experience. Hepatogastroenterology. 2008;55(82-83):592-5.

183. Popescu I, Ciurea S, Romanescu D, Boros M. Isolated resection of the caudate lobe: indications, technique and results. Hepatogastroenterology. 2008;55(84):831-5.

184. Pulitano C, Catena M, Arru M, Guzzetti E, Comotti L, Ferla G, et al. Laparoscopic liver resection without portal clamping: a prospective evaluation. Surg Endosc. 2008;22(10):2196-200.

185. Spencer L, Metcalfe MS, Strickland AD, Elsey EJ, Robertson GS, Lloyd DM. Lessons from laparoscopic liver surgery: a nine-year case series. HPB Surg. 2008;22(1):38-44

186. Troisi R, Montalti R, Smeets P, Van Huysse J, Van Vlierberghe H, Colle I, et al. The value of laparoscopic liver surgery for solid benign hepatic tumors. Surg Endosc. 2008;22(1):38-44.

187. Abu Hilal M, Underwood T, Taylor MG, Hamdan K, Elberm H, Pearce NW. Bleeding and hemostasis in laparoscopic liver surgery. Surg Endosc. 2010;24(3):572-7

188. Al Awad-Jibara A, Chirinos Vargas JR, Baena SJ, Pirela-Finol CA, Brea-Andrade AF, Yajure-Perozo ME. [Laparoscopic hepatectomy in benign solid tumor: case report]. Cir Cir. 2009;77(3):223-7.

189. Bryant R, Laurent A, Tayar C, Cherqui D. Laparoscopic liver resection-understanding its role in current practice: the Henri Mondor Hospital experience. Ann Surg. 2009 Jul;250(1):103-11.

190. Ito K, Ito H, Are C, Allen PJ, Fong Y, Dematteo RP, et al. Laparoscopic versus Open Liver Resection: A Matched-Pair Case Control Study. J Gastrointest Surg. 2009;13(12):2273-83

191. Machado MA, Makdissi FF, Surjan RC, Herman P, Teixeira AR, MC CM. Laparoscopic resection of left liver segments using the intrahepatic Glissonian approach. Surg Endosc. 2009;23(11):2615-9 


\section{Chapter 8}

192. Skalicky T, Treska V, Sutnar A, Liska V, Molacek J, Mirka H, et al. [Surgical treatment of benign liver tumours--indications and results]. Zentralbl Chir. 2009;134(2):141-4.

193. Tomus C, lancu C, Bala O, Graur F, Furcea L, Zaharie F, et al. [Liver resection for benign hepatic lesion: mortality, morbidity and risk factors for postoperative complications]. Chirurgia (Bucur). 2009;104(3):275-80.

194. Watkins J, Balabaud C, Bioulac-Sage P, Sharma D, Dhillon A. Hepatocellular adenoma in advancedstage fatty liver disease. Eur J Gastroenterol Hepatol. 2009;21(8):932-6.

195. Korula J, Yellin A, Kanel G, Campofiori G, Nichols P. Hepatocellular carcinoma coexisting with hepatic adenoma. Incidental discovery after long-term oral contraceptive use. West J Med. 1991;155(4):416-8.

196. Ferrell LD. Hepatocellular carcinoma arising in a focus of multilobular adenoma. A case report. Am J Surg Pathol. 1993;17(5):525-9.

197. Herman P, Machado MA, Volpe P, Pugliese V, Vianna MR, Bacchella T, et al. [Transformation of hepatic adenoma into hepatocellular carcinoma in patients with prolonged use of oral contraceptives]. Rev Hosp Clin Fac Med Sao Paulo. 1994;49(1):30-3.

198. Perret AG, Mosnier JF, Porcheron J, Cuilleron M, Berthoux P, Boucheron S, et al. Role of oral contraceptives in the growth of a multilobular adenoma associated with a hepatocellular carcinoma in a young woman. J Hepatol. 1996;25(6):976-9.

199. Scott FR, el-Refaie A, More L, Scheuer PJ, Dhillon AP. Hepatocellular carcinoma arising in an adenoma: value of QBend 10 immunostaining in diagnosis of liver cell carcinoma. Histopathology. 1996;28(5):472-4.

200. Burri E, Steuerwald M, Cathomas G, Mentha G, Majno P, Rubbia-Brandt L, et al. Hepatocellular carcinoma in a liver-cell adenoma within a non-cirrhotic liver. Eur J Gastroenterol Hepatol. 2006;18(4):437-41. 


\section{Part 4}

Reducing the impact of surgical trauma:

ERAS and laparoscopic surgery 
Part 4 


\section{INTRODUCTION}

In 1992, Gagner and colleagues performed the first laparoscopic liver resection, a wedge resection. Four years thereafter, Azagra et al. reported the first anatomic liver resection. ${ }^{1}$ Initially, laparoscopic liver resection was challenging because of the difficulties concerning safe mobilization and exposure of this fragile and heavy organ. ${ }^{2,3}$ Liver resection is associated with complications such as massive bleeding and bile leakage. ${ }^{2-6}$ Air embolism may occur when high-pressure pneumoperitoneum is used ${ }^{37}$. However, it is suggested that gas embolism is rare and well tolerated. ${ }^{8}$ Other concerns have been raised about the potential dissemination of malignant cells during laparoscopic resection. ${ }^{9,10}$ Some authors have suggested that tumour dissemination has not been increased by laparoscopy. ${ }^{11-13}$ In fact, recent reports regarding this subject show long term survival rates comparable with open surgery. ${ }^{4,14-16}$

In 1995 Cuesta et al. were the first to publish a case report on initial experiences with laparoscopic liver surgery in the Netherlands. ${ }^{17}$ A few years later (2001) a small retrospectively analyzed series $(n=10)$ by the same group was published, showing encouraging results regarding intra-operative bloodloss, postoperative complications and length of hospital stay in wedge- and segment II - III laparoscopic resections. ${ }^{18}$

Encouraged by these results, several liver surgeons in the Netherlands joined forces to enable the safe introduction of this exciting new technique in liver surgery nationwide in a controlled manner.

The following questions will be adressed in this part:

1. Is the introduction of a laparoscopic liver surgery programme for left sided liver lesions possible in a safe manner? Moreover, is it possible to implement this technique for liver tumours without expanded costs?

2. What is the added value of laparoscopic liver resections for solid tumours within an Enhanced Recovery After Surgery (ERAS) programme?

3. What are the results of the initial experience with laparoscopic liver resections in the Netherlands with a focus on factors influencing patient recovery and hence hospital length of stay.

\section{REFERENCES}

1. Azagra JS, Goergen M, Gilbart E, et al. Laparoscopic anatomical (hepatic) left lateral segmentectomy-technical aspects Surg Endosc 1996: 10; 758-761

2. Dagher I, Proske JM, Carloni A, et al. Laparoscopic liver resection: results for 70 patients Surg Endosc 2007: 21; 619-624

3. Cherqui D Laparoscopic liver resection Br J Surg 2003: 90; 644-646 
4. Shimada M, Hashizume M, Maehara S, et al. Laparoscopic hepatectomy for hepatocellular carcinoma Surg Endosc 2001: 15; 541-544

5. Mala T, Edwin B, Rosseland AR, et al. Laparoscopic liver resection: experience of 53 procedures at a single centre J Hepatobiliary Pancreat Surg 2005: 12; 298-303

6. Kaneko H Laparoscopic hepatectomy: indications and outcomes J Hepatobiliary Pancreat Surg 2005: 12 ; 438-443

7. Hashizume M, Shimada M, Sugimachi K Laparoscopic hepatectomy: new approach for hepatocellular carcinoma J Hepatobiliary Pancreat Surg 2000: 7; 270-275

8. Farges $\mathrm{O}$, Jagot $\mathrm{P}$, Kirstetter $\mathrm{P}$, et al. Prospective assessment of the safety and benefit of laparoscopic liver resections J Hepatobiliary Pancreat Surg 2002: 9; 242-248

9. Targarona EM, Martinez J, Nadal A, et al. Cancer dissemination during laparoscopic surgery: tubes, gas, and cells World J Surg 1998: 22; 55-60; discussion 60-51

10. Gutt CN, Riemer V, Kim ZG, et al. Impact of laparoscopic surgery on experimental hepatic metastases Br J Surg 2001: 88; 371-375

11. Cherqui D, Laurent A, Tayar C, et al. Laparoscopic liver resection for peripheral hepatocellular carcinoma in patients with chronic liver disease: midterm results and perspectives Ann Surg 2006: 243; 499-506

12. Laurent $A$, Cherqui $D$, Lesurtel $M$, et al. Laparoscopic liver resection for subcapsular hepatocellular carcinoma complicating chronic liver disease Arch Surg 2003: 138; 763-769; discussion 769

13. Mala T, Edwin B, Gladhaug I, et al. A comparative study of the short-term outcome following open and laparoscopic liver resection of colorectal metastases Surg Endosc 2002: 16; 1059-1063

14. Gigot JF, Glineur D, Santiago Azagra J, et al. Laparoscopic liver resection for malignant liver tumors: preliminary results of a multicentre European study Ann Surg 2002: 236; 90-97

15. Vibert E, Perniceni T, Levard H, et al. Laparoscopic liver resection Br J Surg 2006: 93; 67-72

16. Teramoto K, Kawamura T, Takamatsu S, et al. Laparoscopic and thoracoscopic partial hepatectomy for hepatocellular carcinoma World J Surg 2003: 27; 1131-1136

17. Cuesta MA, Meijer S, Paul MA, et al. Limited laparoscopic liver resection of benign tumors guided by laparoscopic ultrasonography: report of two cases Surg Laparosc Endosc 1995: 5; 396-401

18. Berends FJ, Meijer S, Prevoo W, et al. Technical considerations in laparoscopic liver surgery Surg Endosc 2001: 15; 794-798 


\section{Chapter 9}

\section{The introduction of a laparoscopic liver surgery programme: A cost analysis of initial experience in a university hospital}

J.H.M.B. Stoot, R.M. van Dam, R.J.S. Coelen, B. Winkens, S.W.M. Olde Damink, M.H.A. Bemelmans, C.H.C. Dejong.

Adapted from Scand J Surg 2012;101:32-37

Preliminary results of this study were presented in 2008 to the 8th World Congress of the International Hepato-Pancreatico Biliary Association in Mumbai, India, February 2008, and presented to the 16th International Congress of the European Association for Endoscopic Surgery in Stockholm, Sweden, June 2008. 


\section{ABSTRACT}

Background: In the era of expanding costs of healthcare, this study was conducted to perform a cost analysis of introducing a laparoscopic liver surgery programme for left sided liver lesions.

Methods: Consecutive patients treated by laparoscopic liver resections of left lateral segments were included. Controls were a group of 14 patients undergoing open resection for similar pathology. Primary outcomes were costs. Secondary outcomes were complications, conversions, blood loss, length of operation, and length of hospital stay.

Results: The laparoscopic approach for hepatic left lateral resection (bisegmentectomy 2 and 3) was performed in fourteen patients (group I, median age 54 [range 26-82] years). In the open group, fourteen patients from a prospectively collected database with the same type of resection were selected (group II, median age 64 [range 29-76] years). Costs of theatre usage in the laparoscopic group were significantly lower $(p=0.031)$. No significant differences in costs of disposable instruments, ward stay and total costs were observed between the two groups. There were three complications in the laparoscopic group compared with two complications in the open group. In the laparoscopic group there were 2 conversions (14\%). Median blood loss was significantly lower in the laparoscopic group $(50 \mathrm{mls}$ [range $0-750$ ], $(p=0.001)$ versus the open group (500 mls [range 150-750]). Furthermore, operation time was also significantly lower in the laparoscopic group (116 [range 85-261] minutes) versus the open group (165 [range 96-217] minutes, $p=0.016$ ). Median length of stay was 6 [range 4-11] days in group I versus 6 [range 5-13] days in group II $(p=0.508)$.

Conclusion: Costs of laparoscopic liver resections proved to be equivalent to open surgery. Furthermore, implementation of a laparoscopic liver resection programme seems feasible and safe with reduced blood loss and operation time and comparable morbidity and length of stay. 


\section{INTRODUCTION}

Liver resection remains one of the few procedures where the extended use of laparoscopic techniques has not found its way in everyday practice yet. Several international hospitals have reported their series of laparoscopic liver resections. ${ }^{1-6} \mathrm{Al}-$ though laparoscopic liver resections are gaining popularity world wide, the experience in the Netherlands is still limited. Several studies have reported the feasibility and safety of laparoscopic resections for liver tumours in centres with extensive experience in both hepatobiliary surgery and laparoscopic surgery. ${ }^{1-6}$ The evidence is mostly based on case-series and shows a technique that is reproducible with limited morbidity and mortality. A consensus statement was published on laparoscopic liver resections by Buell J et $a l^{7}$ which makes one of its conclusions that resection of segments 2 and 3 by laparoscopic approach should be the standard of care. However, implementation of new techniques mostly coincides with expanding costs, especially laparoscopic surgery where expensive, disposable tools are required and more operation time is needed..$^{8-10}$ Worldwide, both governmental organisations and health insurance companies have a major influence on cost regulation issues in health care. Hospital managements have budgets for Operation Room (OR) cost and often surgeons are required to make business plans before introducing new techniques. In contrast to many feasibility studies on laparoscopic liver surgery, there is scarce evidence about the cost analysis of this surgical approach. Therefore, the present study was conducted to assess the costs of introducing a laparoscopic liver surgery programme for left sided lesions.

\section{METHODS}

The study describes the first consecutive group of patients undergoing laparoscopic liver resections for solid liver lesions in Maastricht University Medical Centre in the years 2003 to 2008 . This group of patients was compared with a group of patients undergoing the same type of liver resection as an open procedure in a period from 2001 to 2008. Fourteen patients underwent a laparoscopic approach for left lateral resection (group I) and fourteen patients were included in the open group (group II). Preoperative assessment consisted of liver function tests, preoperative radiological evaluation with abdominal ultrasonography, contrast enhanced Computed Tomography (CT) and/or selectively Magnetic Resonance Imaging (MRI) scanning and/or CT PET scanning and multi-disciplinary consensus regarding treatment. All lesions smaller than $10 \mathrm{~cm}$ in diameter and limited to the left lateral liver segments were included. Indications for surgery included presumed benign and malignant liver lesions. The indications for resection were not altered for the minimally invasive group. For patients with presumed benign disease, the indications were pres- 
ence of symptoms, suspicion of a hepatocellular adenoma or cystadenoma or in cases of uncertain nature of the tumour. Indications for liver metastases were synchronous or metachronous liver tumours from colorectal malignancies. Patients were informed about the operative procedure and consent was obtained.

Primary outcomes were costs. Secondary outcomes were complications, conversions, blood loss, length of operation, and length of stay. Complications were defined according to the grading system of Dindo et al. ${ }^{11}$

Unit costs and costs of disposable surgical instruments were obtained retrospectively from the Financial Controller of the Department of Surgery. Costs of ward stay were $€ 225 /$ full day (first day $€ 55$ extra). This was based on rates from 2007 onwards. Costs of theatre use were divided into an hourly rate for surgery and for anaesthesia, which were respectively $€ 190 / \mathrm{h}$ and $€ 560 / \mathrm{h}$. Costs of disposable surgical instruments were as follows: CUSA (Valleylab, Boulder, Colorado, USA) dissecting device €622, Argon plasma coagulator (hand piece) (Bircher Ind., Ltd., Englewood, Colo.USA) €850, Autosuture EndoGIA (Covidien, USA) €256 (reload cartridge $€ 144$ ), Ethicon Ultracision Harmonic ACE (Ethicon Endosurgery, Johnson \& Johnson, USA) €567, Autosuture ENDOBAG (Endocatch Autosuture, Covidien, USA) $€ 99$, 5-12 mm trocar (Versaport Autosuture, Covidien, USA) €73 and $15 \mathrm{~mm}$ trocar (Versaport Autosuture, Covidien, USA) €126. Costs of TachoSil (Nycomed, Zurich, Switzerland) and Tissuecoll (Baxter, Vienna, Austria) for haemostasis were $€ 315$ and $€ 547$ per application, respectively. The number of disposable instruments being used per operation was analysed. After surgery all patients went to the recovery ward for the first 24 hours. The costs of the recovery ward were equal to the general ward. Costs of sterilising of reusable instruments were considered to be equal since almost the same numbers of reusable devices are used for both procedures in our hospital setting. Main endpoints for the cost comparison were costs of theatre usage, disposable surgical instruments and ward stay.

Clinical data were obtained retrospectively from a prospectively collected database. During the selection process, the investigators were unaware of the primary outcome and careful matching was assessed to have similar complexity of procedures and tumour diameter in both groups (table 1). The groups were matched for type of resection and for tumour location and size. The following data were recorded: operation time, blood loss, post-operative complications, histology of the resected liver specimen with diameter of the lesions, resection margins, and hospital length of stay. During the introduction of the laparoscopic liver surgery programme, a multimodal perioperative enhanced recovery programme (ERAS ${ }^{\circledR}$ ) was instituted for all patients undergoing liver surgery in the MUMC and this was also recorded as a parameter. 
Table 1. Clinical and Pathological features.

\begin{tabular}{llll}
\hline & $\begin{array}{l}\text { Group I } \\
\text { Laparoscopic resection } \\
(\mathrm{n}=14)\end{array}$ & $\begin{array}{l}\text { Group II } \\
\text { open resection } \\
(\mathrm{n}=14)\end{array}$ & p-value \\
\hline Sex ratio, M / F & $3 / 11$ & $9 / 5$ & 0.054 \\
Age median (range) & $54(26-82)$ & $64(29-76)$ & 0.579 \\
ASA 1 & 4 & 2 & 0.529 \\
ASA 2 & 8 & 8 & \\
ASA 3 & 2 & 4 & 0.128 \\
Malignant/benign & $5 / 9$ & $10 / 4$ & 0.408 \\
Tumour size in cm, median (range) & $4.1(1.0-10.5)$ & $2.5(1.2-10.5)$ & $>0.999$ \\
Resection margins (R0/R1/R2/NR*) & $12 / 1 / 1$ & $11 / 1 / 1 / 1$ & 0.236 \\
Traditional care / ERAS ${ }^{\circledR}$ & $3 / 11$ & $7 / 7$ & \\
\hline
\end{tabular}

No statistical significant differences were observed between both groups. ${ }^{*} \mathrm{NR}=$ not reported.

All patients were carefully followed after discharge according to the MUMC liver surgery protocol: patients were followed periodically in the first, third, sixth and twelfth postoperative months in the first year and at least twice a year thereafter. The follow-up procedure included physical examination, laboratory tests, ultrasonography of the abdomen, and CT scanning in cases where this was considered necessary.

\section{Surgical technique}

The laparoscopic procedures were performed by two consultant surgeons; one expert in hepatobiliary surgery and one with specific expertise in advanced laparoscopic surgery. Prior to the first liver resections, surgeons visited expert centres in laparoscopic liver surgery outside the Netherlands. Liver resections were defined according to anatomical planes (Couinaud's classification). Only lesions in the left lateral segments requiring anatomical resection of segment II and III were included in the laparoscopic resection group. In the laparoscopic approach group, patients were placed in the supine position under general anaesthesia, with lower extremities apart. ${ }^{6}$ The surgeon was standing between the legs with an assistant on each side. An open introduction of the subumbilical port was used to produce the $\mathrm{CO}_{2}$ pneumoperitoneum. Abdominal pressure was monitored at $10-12 \mathrm{mmHg}$ and a $30^{\circ}$ laparoscope was used in all cases. Four extra trocars were placed to facilitate the procedure. ${ }^{6}$ The liver was inspected visually and laparoscopic-ultrasound was used in all cases to confirm the number of lesions in relation to the main anatomical structures. The round, falciform and left triangular ligaments and the lesser omentum were divided. Hepatic transection of parenchyma and minor crossing vessels and biliary radicals was performed with harmonic scalpel (Ultracision, Ethicon Endosurgery, Johnson \& Johnson, USA) without the use of a clamping method (Prin- 
gle's manoeuvre). The parenchyma transection with this device is reported to be sufficient for vascular and biliary structures up to $3 \mathrm{~mm} .{ }^{6}$ The portal pedicles and hepatic veins were stapled using a vascular stapler (EndoGIA Autosuture, Covidien, USA). Resected specimens were placed in a plastic bag (Endocatch Autosuture, Covidien, USA) and removed through a separate suprapubic incision or, in cases where a laparotomy had been performed in the past, through a small subumbilical or subcostal incision. No hand-access devices were used for the laparoscopic approach.

For the open approach, a bilateral subcostal incision was mostly used to enter the abdomen. After abdominal exploration in search of extrahepatic disease, the round, falciform and left triangular ligaments and the lesser omentum were divided. The liver was inspected visually and intra operative ultrasound was performed in all cases to confirm the number of lesions in relation to the main anatomical structures. Hepatic transection of parenchyma was done using the Cavitron Ultrasonic Surgical Aspirator (CUSA ${ }^{\circledR}$; Valleylab, Boulder, Colorado, USA) and argon beam coagulator (Bircher Ind., Ltd., Englewood, Colo.USA) with or without the use of Pringle's manoeuvre. Minor crossing vessels and biliary radicals were divided using polypropylene sutures or clips. The portal pedicles and hepatic veins were divided and ligated with a running polypropylene suture. After removal of the liver specimen, the raw surface of the liver remnant was subjected to argon beam coagulation and sealed with TachoSil ${ }^{\circledR}$ (Nycomed, Zurich, Switzerland) or Tissuecoll (Baxter, Vienna, Austria) if appropriate. ${ }^{12}$

\section{Statistial analysis}

Continuous variables were expressed as median (range). Statistical analysis of cost comparison was performed using a Mann Whitney $U$ Test, since the groups were small and a non-normal distribution in both groups was assumed. Costs were ex pressed as mean ( $\pm S D$ ), to be able to compare the results from this cost analysis with other studies. Data were analysed according to the intention to treat principle. The Chi-square test (or Fisher exact test where appropriate) was used to investigate differences in demographic parameters: sex, ASA classification, pathology, resection margins, perioperative care programme and the primary outcome parameter complications. Mann-Whitney $U$ Test was used to investigate differences in age, tumour diameter, duration of operation ( $\mathrm{min}$.), blood loss ( $\mathrm{mls}$ ) and hospital length of stay (days). A p-value of $\leq 0.05$ was considered to indicate statistical significance. SPSS statistical software (version 15.0, Chicago, Illinois, U.S.A.) was used for the analysis. 


\section{RESULTS}

The laparoscopic approach for left lateral resection (bisegmentectomy 2 and 3) was performed in fourteen patients and completed laparoscopically in twelve patients (table 1). In the open group, fourteen patients were included (group II). Demographics of both groups are reported in table 1 . There were no significant differences in sex, age, ASA classification, benign lesions, tumour size, resection margins and perioperative care programme.

Costs of theatre use were significantly higher in the open group (table 2). The costs of disposable surgical instruments were on average €206 per patient lower for laparoscopic surgery. No significant differences were observed in ward stay. Overall, total costs tended to be on average $€ 611$ per patient lower in the laparoscopic group, but this failed to attain statistical significance (table 2).

Table 2. Primary outcome: Cost analysis using the Mann-Whitney U Test.

\begin{tabular}{|c|c|c|c|c|}
\hline & $\begin{array}{l}\text { Group I } \\
\text { Laparoscopic } \\
(n=14)\end{array}$ & $\begin{array}{l}\text { Group II } \\
\text { Open } \\
(n=14)\end{array}$ & Difference & $\mathrm{p}$-value \\
\hline Theatre usage (mean \pm SD) & $€ 2530 \pm 619$ & $€ 2984 \pm 662$ & $-€ 453$ & 0.031 \\
\hline Disposable instruments (mean \pm SD) & $€ 1760 \pm 406$ & $€ 1966 \pm 281$ & $-€ 206$ & 0.145 (n.s.) \\
\hline Ward stay (mean \pm SD) & $€ 1678 \pm 425$ & $€ 1630 \pm 499$ & $+€ 48$ & 0.508 (n.s.) \\
\hline Total (mean \pm SD) & $€ 5969 \pm 1112$ & $€ 6580 \pm 922$ & $-€ 611$ & 0.062 (n.s.) \\
\hline
\end{tabular}

There was one grade I complication and two grade II complications in the laparoscopic group (table 3). One patient remained longer in hospital due to constipation, one patient had a urinary tract infection and another patient was treated for atrial fibrillation. In the open group there was one grade I complication and also one grade II complication. One patient had a delayed functioning of the gastrointestinal tract and one patient was treated for a myocardial infarction. Respectively, eleven and twelve patients in each group had an uneventful recovery. The two conversions (14\%) were due to tumour size, additional unanticipated lesions and close relation with the left liver vein. One patient appeared to also have a lesion in segment IV and the exposure was considered insufficient to perform a safe resection. The second conversion was performed because of the close relation to the left hepatic vein, large diameter of the lesion and uncertain nature of the tumour. Median blood loss was significantly higher in the open group than in the laparoscopic group ( $p<001$ ). Duration of operation was also significantly reduced in the laparoscopic group $(p=0.012)$. The data on secondary outcomes are presented in table 3. There were no deaths within 30 days after surgery. 
Table 3. Secondary outcomes.

\begin{tabular}{llll}
\hline & $\begin{array}{l}\text { Group I } \\
\text { Laparoscopic resection } \\
(\mathrm{n}=14)\end{array}$ & $\begin{array}{l}\text { Group II } \\
\text { Open resection } \\
(\mathrm{n}=14)\end{array}$ & p-value \\
\hline $\begin{array}{l}\text { Primary outcome } \\
\text { Complications (grade) }\end{array}$ & $3(\mathrm{I}, \mathrm{II})$ & $2(\mathrm{I}, \mathrm{II})$ & $>0.999$ \\
$\begin{array}{l}\text { Secondary outcome (medain) } \\
\text { Conversions }\end{array}$ & $2(14 \%)$ & & \\
$\begin{array}{l}\text { Blood loss (ml) } \\
\text { Operation time (minutes) } \\
\text { Length of stay (days) }\end{array}$ & $\begin{array}{l}116 \text { [range 0-750] } \\
6 \text { [range 4-11] }\end{array}$ & 500 [range 150-750] & 0.001 \\
\hline
\end{tabular}

In the laparoscopic group there were 9 benign lesions and 6 malignant tumours ( 5 patients). The median tumour size was 4.1 [range 1-10.5] $\mathrm{cm}$ (table 1). In this group, there were three adenomas, three focal nodular hyperplasia (FNH), an angiolipoma, a hemangioma, a presumed metastases, which proved to be an inflammatory pseudotumor after chemotherapy and 6 metastases of colorectal origin. All but one resection margins were free of disease; the $\mathrm{R} 2$ resection was found in a resected specimen of an adenoma. In the open group, there were six benign lesions (two adenomas, FNH, polycystic liver disease, inflammatory granuloma, and focal hemorrhage; the latter two in patients with malignant disease) and 10 malignant tumours in eight patients (mostly colorectal metastases, and one sarcoma) with a median tumour size of 2.5 [range $1.2-10.5$ ] cm (table 1). In all but one patients, an R0 resection was performed.

In group I, there were no late complications specifically related to the laparoscopic procedure within a median follow-up of 43 [range 12-97] months. No portsite metastases were detected in this group. However, 5 patients died during follow-up after $12(n=2), 27,32$ and 37 months. Causes of death of these patients were cancer related; all patients died due to progressive metastastic disease. Concerning the late complications in the open group, there were two symptomatic incisional hernias within the median follow-up of 60 [range 11-128] months. In the open group, six patients died during follow-up after $11(n=2), 14,28,39$ and 87 months. All but one of these patients died with progressive metastastic disease. One patient died due to a cancer unrelated cause.

\section{DISCUSSION}

Laparoscopic surgery is considered to be more expensive compared to open procedures, especially because most laparoscopic procedures need more operating time and more disposable devices than their open counterparts. ${ }^{8-10}$ Although perioperative outcome remains the surgeon's main focus, the costs of the procedure should 
also be accounted for when deciding between a laparoscopic or open approach. The use of disposable surgical instruments and prolonged operation room-usage consequently may lead to an increase in costs. We therefore performed a cost analysis, calculating the costs of laparoscopic and open liver resections. The results of this cost analysis shows that, apart from significantly lower costs of theatre usage in the laparoscopic group, no statistically significant differences in costs of disposable instruments, ward stay and total costs were observed between the laparoscopic and open group.

The present results are favourably comparable with a recent study on this subject, where no differences were found in operation time and costs. ${ }^{13}$ Other reports showed that the total costs for laparoscopic surgery were significantly lower. ${ }^{14,15}$ In these studies, a considerable difference was seen in hospital days, which consequently led to lower costs in the laparoscopic groups. In the present study no difference in length of hospital stay was observed between both groups. Moreover, both CUSA and argon beam coagulator are used in the MUMC for the open resections which leads to considerable costs of disposables, in contrast to the laparoscopic approach where only the harmonic scalpel is used for the liver transection.

This study confirms previous reports in the literature that the introduction of a new technique such as laparoscopic resection of solid liver tumours in segment II and III is feasible and safe. It was suggested that laparoscopy is a safe approach in a selected group of patients with reduced blood loss and reduced morbidity., ${ }^{5,16-20}$ To date in the era of evidence based medicine, however, there is scarce high-level evidence that properly compares laparoscopic liver resection and open hepatic resection. There are no randomised studies available to confirm the observations in the prospective and retrospective studies hitherto reported. Some prospective case-series studies have been published with similar conclusions of reduced blood loss and morbidity. ${ }^{14,18,21}$ However, apart from the previously mentioned advantages, most investigators observed a longer operation time and have postulated increased costs of laparoscopic surgery.

In the present attempt to study the effects of laparoscopic liver surgery in a comparative setting, we observed a faster resection, less intra-operative blood loss with comparable morbidity, both in the short and long term. The reduced blood loss might be caused by the raised intra-abdominal pressure of the pneumoperitoneum which is necessary for the laparoscopic approach. This may result in a tamponade of low-pressure venous oozing during the procedure, as previously suggested by others. ${ }^{13}$ Also, different transection devices during the procedure might also reduce the need for prolene stitches and haemostatic clips which in some cases can be time consuming. In this study of initial experience of laparoscopic hepatic resections, the conversion rate of $14 \%$ was in concordance with previous reports of $1-26 \% .{ }^{1-4,6,16}$ Both conversions were performed after the use of laparoscopic ultrasound to determine whether a safe resection was possible. This study 
was not designed for long-term outcome; but no long-term complications were observed in the laparoscopic group in contrast with two symptomatic incisional hernias in the open group.

It is interesting to observe that there were no significant differences in hospital stay between the open and laparoscopic approach. Striking is the relatively long stay in hospital for the laparoscopic procedures. This was previously also observed for colonic resections before and after the start of a perioperative multimodal fast track setting (Enhanced Recovery After Surgery; ERAS ${ }^{\circledR}$ ). ${ }^{22}$ Although in this recent study predefined discharge criteria were assessed, almost $90 \%$ of the ERAS patients were not going home on the day the discharge criteria were actually met. After careful investigation for valid reasons of prolonged stay, $69 \%$ of this extended hospital stay was labelled as inappropriate. Only a minority was rated as medically necessary. Similar observations of prolonged length of stay after the discharge criteria are fulfilled, were also reported in the first study on fast track laparoscopic liver surgery. ${ }^{23}$

The oncologic principle outweighs the assumed benefits of the laparoscopic technique such as rapid recovery, short postoperative stay and cosmetic aspect. Improvement of overall survival remains the ultimate goal for resection of liver metastases and should not be compromised for the functional postoperative advantages of the minimally invasive approach. Radical resection, tumour free margins and the 'no touch' technique are mandatory. ${ }^{2,24}$ This is effectuated with the use of extraction site protections and endo-bags for the specimens. Also, intraoperative ultrasound is reported to be one of the most important factors to reduce positive resection margins and it helps avoiding injury to deeply seated lesions. ${ }^{2}$ This can be performed laparoscopically to improve outcome $e^{1,25}$ and its routine use should be advocated. ${ }^{2}$ An additional advantage of the laparoscopic approach in combination with the laparoscopic ultrasound is the avoidance of unnecessary exploratory laparotomy in cases with peritoneal metastases or otherwise irresectable disease. ${ }^{26-28}$

Results of long term follow up amongst others in patients with cancer are required to determine the exact place of laparoscopic liver resection in treating patients with solid liver tumours. The data from other studies on laparoscopically resected liver tumours show no difference in oncologic clearance and similar overall and disease free survival rates as in open surgery. ${ }^{1,29,30}$ Laparoscopic liver resections proved to be faster, which is remarkable to some extent as most laparoscopic procedures require more time than their open counterparts. The reasons for this are speculative but may relate to the use of different equipment. This may be a valid subject for a randomised controlled trial.

The indication for laparoscopic liver resection should not be different from an open resection: benign abnormalities as symptomatic liver cysts, hepatocellular adenoma, symptomatic focal nodular hyperplasia, symptomatic haemangioma and 
malignancies such as hepatocellular carcinomas (HCC) and liver metastases., ${ }^{1,2,5,7}$ In this study, the preoperative indications for the minimally invasive technique were malignancy, symptomatic benign solid lesion or uncertain nature of the tumour requiring excision. The choice for the open or laparoscopic approach is mainly determined by the size and location of the lesion and the experience of the surgical team.

\section{Conclusions}

The introduction of the laparoscopic technique for left lateral hepatic lesions is equivalent to the open technique with regard to total costs and is safe, feasible, and can be used for diagnosis, preoperative staging and finally resection. The indication for operating benign lesions should not be changed, however, the benefits of the laparoscopic technique especially nominate the use in a selected group of patients with symptomatic benign lesions, uncertain diagnosis or suitable hepatic locations of malignancies.

Acknowledgements: We thank T. Lodewick and E. Wong-Lun-Hing of the Department of Surgery of the MUMC, for their kind assistance in retrieving the long-term follow-up data.

\section{REFERENCES}

1. Gigot JF, Glineur D, Santiago Azagra J, et al. Laparoscopic liver resection for malignant liver tumors: preliminary results of a multicentre European study. Ann Surg 2002;236:90-97

2. Dulucq JL, Wintringer $P$, Stabilini $C$, Berticelli J, Mahajna A. Laparoscopic liver resections: a single centre experience. Surg Endosc 2005;19:886-891

3. Descottes B, Glineur D, Lachachi F, et al. Laparoscopic liver resection of benign liver tumors. Surg Endosc 2003;17:23-30

4. Descottes B, Lachachi F, Sodji M, et al. Early experience with laparoscopic approach for solid liver tumors: initial 16 cases. Ann Surg 2000;232:641-645

5. Dagher I, Proske JM, Carloni A, et al. Laparoscopic liver resection: results for 70 patients. Surg Endosc 2007;21:619-624

6. Cherqui D, Laurent A, Tayar C, et al. Laparoscopic liver resection for peripheral hepatocellular carcinoma in patients with chronic liver disease: midterm results and perspectives. Ann Surg 2006;243:499-506

7. Buell JF, Cherqui D, Geller DA, et al. The international position on laparoscopic liver surgery: The Louisville Statement, 2008. Ann Surg 2009;250:825-830

8. Braga M, Frasson M, Vignali A, et al. Laparoscopic vs. open colectomy in cancer patients: long-term complications, quality of life, and survival. Dis Colon Rectum 2005;48:2217-2223

9. Murray A, Lourenco T, de Verteuil R, et al. Clinical effectiveness and cost-effectiveness of laparoscopic surgery for colorectal cancer: systematic reviews and economic evaluation. Health Technol Assess 2006;10:1-141, iii-iv 


\section{Chapter 9}

10. McCormack K, Scott NW, Go PM, Ross S, Grant AM. Laparoscopic techniques versus open techniques for inguinal hernia repair. Cochrane Database Syst Rev 2003:CD001785

11. Dindo D, Demartines N, Clavien PA. Classification of surgical complications: a new proposal with evaluation in a cohort of 6336 patients and results of a survey. Ann Surg 2004;240:205-213

12. van Dam RM, Hendry PO, Coolsen MM, et al. Initial experience with a multimodal enhanced recovery programme in patients undergoing liver resection. Br J Surg 2008;95:969-975

13. Rowe AJ, Meneghetti AT, Schumacher PA, et al. Perioperative analysis of laparoscopic versus open liver resection. Surg Endosc 2009;23:1198-1203

14. Polignano FM, Quyn AJ, de Figueiredo RS, et al. Laparoscopic versus open liver segmentectomy: prospective, case-matched, intention-to-treat analysis of clinical outcomes and cost effectiveness. Surg Endosc 2008;22:2564-2570

15. Vanounou T, Steel JL, Nguyen KT, et al. Comparing the clinical and economic impact of laparoscopic versus open liver resection. Ann Surg Oncol;17:998-1009

16. Cherqui $D$, Husson $E$, Hammoud $R$, et al. Laparoscopic liver resections: a feasibility study in 30 patients. Ann Surg 2000;232:753-762

17. Laurence JM, Lam VW, Langcake ME, et al. Laparoscopic hepatectomy, a systematic review. ANZ J Surg 2007;77:948-953

18. Lesurtel M, Cherqui D, Laurent A, Tayar C, Fagniez PL. Laparoscopic versus open left lateral hepatic lobectomy: a case-control study. J Am Coll Surg 2003;196:236-242

19. Mala T, Edwin B, Gladhaug I, et al. A comparative study of the short-term outcome following open and laparoscopic liver resection of colorectal metastases. Surg Endosc 2002;16:1059-1063

20. Simillis C, Constantinides VA, Tekkis PP, et al. Laparoscopic versus open hepatic resections for benign and malignant neoplasms--a meta-analysis. Surgery 2007;141:203-211

21. Troisi $R$, Montalti $R$, Smeets $P$, et al. The value of laparoscopic liver surgery for solid benign hepatic tumors. Surg Endosc 2008;22:38-44

22. Maessen JM, Dejong $\mathrm{CH}$, Kessels AG, von Meyenfeldt MF. Length of stay: an inappropriate readout of the success of enhanced recovery programmes. World J Surg 2008;32:971-975

23. Stoot JH, van Dam RM, Busch OR, et al. The effect of a multimodal fast-track programme on outcomes in laparoscopic liver surgery: a multicentre pilot study. HPB (Oxford) 2009;11:140-144

24. Teramoto K, Kawamura T, Takamatsu S, et al. Laparoscopic and thoracoscopic partial hepatectomy for hepatocellular carcinoma. World J Surg 2003;27:1131-1136

25. John TG, Greig JD, Crosbie JL, Miles WF, Garden OJ. Superior staging of liver tumors with laparoscopy and laparoscopic ultrasound. Ann Surg 1994;220:711-719

26. D'Angelica $\mathrm{M}$, Fong $\mathrm{Y}$, Weber $\mathrm{S}$, et al. The role of staging laparoscopy in hepatobiliary malignancy: prospective analysis of 401 cases. Ann Surg Oncol 2003;10:183-189

27. Metcalfe MS, Close JS, Iswariah $\mathrm{H}$, et al. The value of laparoscopic staging for patients with colorectal metastases. Arch Surg 2003;138:770-772

28. Rahusen FD, Cuesta MA, Borgstein PJ, et al. Selection of patients for resection of colorectal metastases to the liver using diagnostic laparoscopy and laparoscopic ultrasonography. Ann Surg 1999;230:31-37

29. Gayet B, Cavaliere D, Castel B, et al. [Laparoscopic liver surgery for metastases of colorectal cancer: analysis of a monocentric experience]. Suppl Tumori 2005;4:S135-137

30. Vibert E, Perniceni T, Levard H, et al. Laparoscopic liver resection. Br J Surg 2006;93:67-72 


\section{Chapter 10}

\section{The effect of a multimodal 'fast track' programme on outcomes in laparoscopic}

liver surgery: A multicentre pilot study

J.H.M.B. Stoot, R.M. van Dam, O.R. Busch, R. van Hillegersberg, M. de Boer, S.W.M. Olde Damink, M.H.A. Bemelmans, C.H.C. Dejong.

On behalf of the Enhanced Recovery After Surgery (ERAS) Group

Adapted from HPB 2009; 11: 140-144

Parts of this study were presented to the $8^{\text {th }}$ World Congress of the International Hepato-Pancreato-Biliary Association, Mumbay, India, February 2008, and to the International Surgical Congress of the Association of Surgeons of Great Britain and Ireland, Bournemouth, England, May 2008. 


\section{ABSTRACT}

Background: This study was conducted to evaluate the added value of an Enhanced Recovery After Surgery (ERAS) programme for laparoscopic liver resections for solid tumours.

Methods: Patients undergoing laparoscopic liver resection between July 2005 to July 2008 were included. Indications for resections included presumed benign and malignant liver lesions. Primary outcome was total length of hospital stay (LOS). Secondary outcomes were functional recovery, complications, conversions, blood loss and duration of operation.

Results: Thirteen patients were treated by laparoscopic liver resections in the ERAS programme in one centre (group 1). These were compared with thirteen laparoscopic procedures performed either before introduction of the ERAS programme from 2003-2005 in that centre or in the same era in other centres with traditional care (group 2). Median total LOS was 5.0 days [range 3-10 days] in group 1 versus 7.0 days [3-12 days] in group 2. This difference was not statistically significant. Functional recovery occurred two days earlier in group 1 (median 3.0 days [1-7 days] versus 5.0 days [2-8 days], $P<0.044)$. There were no significant differences in complications, conversions or duration of operation. Blood loss was significantly less in the ERAS group (median $50 \mathrm{ml}$ [range 50-200 ml] vs. median $250 \mathrm{ml}$. [range $50-800 \mathrm{ml}], P<0.002)$.

Conclusions: This exploratory, multicentre, fast-track laparoscopic liver resection study is the first such study conducted. Although small, the study suggests that a multimodal enhanced recovery programme for laparoscopic liver surgery is feasible, safe and may lead to accelerated functional recovery and reduction in LOS. 


\section{INTRODUCTION}

Laparoscopic surgical procedures have been popularized because they allow for rapid recovery, shorter postoperative hospital stays and improved cosmetic outcomes. In gastrointestinal surgery, the minimally invasive approach is extensively used in many operations and has become the gold standard for some of these procedures, such as cholecystectomy and adrenalectomy. ${ }^{1,2}$ For solid tumours in the liver, the laparoscopic technique is used more and more frequently in expert centres and the results, mostly from cohort studies, suggest benefits, notably in terms of shorter postoperative stays. ${ }^{3}$ With respect to improved recovery and outcome, a multimodal enhanced recovery after surgery (ERAS) programme has recently shown promising results in many open elective colorectal operations, as well as in liver surgery. ${ }^{4,5}$ This multimodal recovery programme is evidence based and combines several interventions in perioperative care to reduce the stress response and organ dysfunction with a focus on enhancing recovery. ${ }^{5,6}$ The key elements of this protocol include the provision of preoperative counselling, perioperative intravenous (i.v.) fluid restriction, optimal pain relief, preferably without the use of opioid analgesia, early oral nutrition and enforced mobilisation, and the absence of nasogastric tubes and drains. ${ }^{6-8}$ In other fields of elective surgery, similar programmes have been shown to reduce hospital stay by several days. ${ }^{9,10}$ However, to our knowledge, the added value of a fast-track ERAS programme in laparoscopic liver surgery has hitherto not been explored. At the Maastricht University Medical Centre (MUMC), the ERAS programme for liver surgery started 2 years after the laparoscopic programme for left lateral sectionectomies. Therefore, a pilot study was conducted to find out whether the ERAS programme was feasible and safe and whether it improved recovery in the laparoscopic setting for liver surgery.

\section{METHODS}

Patients undergoing laparoscopic liver resections for solid liver tumours between July 2003 and July 2008 were enrolled in the study. Preoperative assessment consisted of standard plasma liver function tests, preoperative radiological evaluation with abdominal ultrasonography, contrast-enhanced computed tomography (CT) and/or selective magnetic resonance imaging (MRI) scanning and/or CT-positron emission tomography (CT-PET). Multidisciplinary consent was also required. All lesions $<10 \mathrm{~cm}$ in diameter and judged amenable to laparoscopic resection were included (left lateral sectionectomy or antero lateral small lesions of the right side of the liver). All but one lesion were limited to lateral liver segments (II, III, V, VI). One lesion was located centrally in segment IV. Indications for surgery included presumed benign and malignant liver lesions. For patients with presumed benign 
disease, the indications were suspicion of a hepatocellular adenoma or cystadenoma or, in case where the nature of the tumour was uncertain, the presence of symptoms and risk of bleeding. Indications for liver metastases were synchronous or metachronous liver tumours from colorectal malignancies. Patients were informed about the operative procedure and consent was obtained.

The ERAS perioperative programme was instituted in the surgical unit in Maastricht at the beginning of 2002, initially for patients undergoing elective colonic resections. ${ }^{11}$ The protocol for this multimodal, evidence-based, enhanced recovery programme for colonic surgery requires extensive preoperative counselling, no premedication, no preoperative fasting but carbohydrate containing liquids until 2 hours before surgery, mid-thoracic epidural anaesthesia and short-acting anaesthetics, perioperative oxygen supplementation, avoidance of perioperative fluid overload, non-opoid pain management, no routine use of nasogastric tubes, early removal of bladder catheters, standard laxatives and prokinetics, and early and enhanced postoperative feeding and mobilization. ${ }^{5,11}$ This protocol was modified in the year 2005 to cover all aspects of liver surgery, including laboratory liver function tests, and to avoid excessive fluids (preoperative and peroperative central venous pressure $[\mathrm{CVP}]<5 \mathrm{mmHg})^{4}$

Until May 2005, traditional care was given before, during and after liver surgery. Therefore, the 5-year period during which laparoscopic liver surgery for solid tumours has been performed at MUMC was divided into two periods, representing the 2 years before the ERAS programme was implemented and the 3 years immediately following its implementation. As the numbers of patients in the MUMC control group (patients undergoing surgery with traditional perioperative care) was too small to allow us to draw firm conclusions, other major liver centres in the Netherlands, which were not familiar with the ERAS programme and which applied a traditional care protocol were contacted in order to enroll additional laparoscopic liver resection patients. These included the Academical Medical Centre (AMC), Amsterdam; the University Medical Centre Utrecht (UMCU), Utrecht and the University Medical Centre Groningen (UMCG), Groningen.

The following recovery data were defined (and patients were considered functionally recovered when all criteria were met): normal or decreasing serum bilirubin; good pain control on oral analgesics only; absence of i.v. fluids and tolerance of solid food, and mobilization to preoperative level. ${ }^{4,8}$ Patients were considered fit for discharge, or functionally recovered, on the postoperative day when all four criteria were met. Patients were discharged if all four criteria were met and if they were willing to go home. ${ }^{4}$ A discharge that occurred later than functional recovery was defined as a delayed discharge. 
Primary outcome was total length of hospital stay (LOS), defined as the number of nights spent in hospital after surgery, including nights after readmission within 90 days of surgery. Secondary outcomes were functional recovery, complications, conversions, blood loss, and length of operation. In addition, the following data were recorded: preoperative indication(s) for surgery; perioperative blood transfusions; use of nasogastric tubes; use of drains; restart of oral fluids; restart of oral solid food; histology of the resected liver specimen, and diameter of the lesions and resection margins.

Complications were defined according to the grading system by Clavien et al. ${ }^{12}$ Grade I complications are generally non-life-threatening complications without lasting disability. Grade II are potentially life-threatening, but without residual disability. Grade III complications involve residual disability, including organ resections or persistence of life-threatening conditions. Grade IV complications refer to deaths as a result of complications.

All laparoscopic hepatic resections were performed by two consultant surgeons, one expert in hepatobiliary surgery and one with specific expertise in advanced laparoscopic surgery. Patients were placed in the 'French' position (supine position with both legs apart) under general anesthesia. The surgeon stood between the legs with an assistant on each side. ${ }^{13}$ Liver resections were defined according to anatomical planes (Couinaud's classification). An open introduction of the subumbilical port was used to produce the $\mathrm{CO}_{2}$ pneumoperitoneum. In all cases, a $30^{\circ}$ laparoscope was used and the abdominal pressure was monitored and maintained at 12-14 $\mathrm{mmHg}$. Three to four extra trocars were placed in a semi-circle, slightly higher and paramedian of the umbilical trocar, to facilitate the procedure. ${ }^{13}$ Inspection of the liver was performed both visually and with laparoscopic-ultrasound in order to confirm the number of lesions in relation to main anatomical structures. The falciform, left triangular ligament and the lesser omentum were divided. Hepatic transection of parenchyma and minor crossing vessels and biliary radicals was performed with harmonic scalpel (Ultracision; Ethicon Endo-Surgery, Johnson \& Johnson, Piscataway, NJ, USA). A vascular stapler (EndoGIA; Covidien Surgical Devices, New Haven, CT, USA) was used to divide the portal pedicles and hepatic veins. Resected specimens were placed in a plastic bag (Endocatch; Covidien Surgical Devices) and removed through a separate, preferably suprapubic incision. In cases where a laparotomy had been performed in the past, a small subumbilical incision was made for retrieval of the specimen.

\section{Statistical analysis}

Continuous variables are expressed as median (range). Age, tumour diameter, hospital stay, functional recovery, delay in discharge, blood loss and duration of sur- 
gery were analysed using the Mann-Whitney $U$-test. Sex, American Society of Anesthesiology (ASA) classification, indication for surgery, type of resection, liver pathology, complications, conversions, intake of fluid and solid food and the use of nasogastric tubes and drains were analysed using the Chi-square or Fisher's exact test as appropriate. A $P$-value $<0.05$ was considered to be statistically significant. Data were analysed using spss ${ }^{\circledR}$ version 13.0 for Windows ${ }^{\circledR}$ (SPSS, Inc.,Chicago, IL, USA).

\section{RESULTS}

A total of 26 patients were treated by laparoscopic liver resection during the 5year period in the four participating hospitals. Thirteen patients were treated by laparoscopic liver resections (left lateral sectionectomy (II, III) or segmentectomy (IV, V, VI) under the ERAS programme (group 1). All of these were operated at a single centre (Maastricht $U M C$ ). Thirteen patients were operated in traditional perioperative care setting in the four participating hospitals during the same period (group 2). Three laparoscopic procedures were performed at MUMC from 20032005 before the ERAS programme was instituted. Ten patients were operated laparoscopically in a traditional perioperative care setting in the AMC $(n=4)$, UMCU ( $=2)$ and UMCG ( $n=4)$.

Demographics and clinical data are reported in table 1. Median total LOS was reduced by two days in the ERAS group, but this difference did not attain statistical significance (table 2). There were no readmissions in either group. There were two minor complications (grade I) in the ERAS group and two (grade I) in the traditional care group. In group 1, one patient had a urinary tract infection, which was treated by antibiotics, and another had constipation and remained in hospital longer. In group 2, one patient was treated for a hypokalaemia and another for atrial fibrillation. There were no deaths. The rate of conversions was similar in both groups (table 2). In group 2 (traditional care), conversion occured because of a preoperatively undetected lesion in segment IV in one patient and suboptimal visualization in another. The conversions in the ERAS group occured because of the uncertain nature and size of the lesion and its proximity to the left liver vein in one patient and because of suboptimal visualization in another. Blood loss was significantly less in the ERAS group $(P<0.002)$. However, there was no difference in blood transfusions. Only one patient from group 2 (traditional care) received two units of packed cell while in hospital. No significant difference was observed in the operation time. Oral fluid intake was resumed within 24 hours after surgery in almost all patients of the ERAS group, as well as in the traditional care group [median 1 day (range 0-2 days) vs. median 1 day (range 0-6 days), respectively; $P=0.861$ ]. The median time to successful resumption of normal diet was 1 day (range 1-2 days) in 
the ERAS group, compared with 2 days (range $1-6$ days) in the controls $(P=0.223$ ). Nasogastric decompression tubes were not used after surgery in the ERAS group. In the control group, nasogastric tubes were in place after surgery in five of 13 patients for 1-5 days $(P=0.257)$. This included two reinsertions because of delayed gastrointestinal function on day 1 . In the ERAS group, no drains were used after surgery. In the control group, six patients received a drain, which was removed 2-4 days after surgery $(P=0.015)$.

Table 1. Clinical and pathological features.

\begin{tabular}{|c|c|c|c|}
\hline & $\begin{array}{l}\text { Group I } \\
\text { ERAS programme } \\
(\mathrm{n}=13)\end{array}$ & $\begin{array}{l}\text { Group II } \\
\text { Traditional care } \\
(n=13)\end{array}$ & $P$-value \\
\hline$\overline{\text { Age } \text { (years) }^{a}}$ & $55[34-82]$ & $45[26-70]$ & $0.104^{b}$ \\
\hline ASA score I / II / III / IV & $3 / 9 / 1 / 0$ & $6 / 6 / 1 / 0$ & 0.688 \\
\hline Patient sex, M / F & $3 / 10$ & $2 / 11$ & 1.0 \\
\hline Indication for surgery: malignant / benign & $8 / 5$ & $3 / 10$ & 0.111 \\
\hline \multicolumn{4}{|l|}{ Types of liver resection } \\
\hline Left lateral sectionectomy & 9 & 9 & \\
\hline Monosegmentectomy (or metastasectomy) & 3 & 3 & 0 \\
\hline Multiple segmentectomy ( $\geq 2$ segments) & 1 & 1 & \\
\hline Liver Pathology: malignant / benign & $5 / 8$ & $2 / 11$ & 0.160 \\
\hline Tumour size $(\mathrm{cm})^{\mathrm{a}}$ & $4.1[1-10.5]$ & $6.0[1.4-8]$ & $0.765^{b}$ \\
\hline
\end{tabular}

${ }^{a}$ Continuous variables are expressed as median [range].

P-values by Fisher's exact test, except ${ }^{\mathrm{b}}$ Mann-Whitney $U$ test.

ERAS, Enhanced Recovery After Surgery; ASA, American Society of Anaesthesiologists; M, male; F, female

Table 2. Primary and secondary outcomes. No readmission were recorded in either groups.

\begin{tabular}{llll}
\hline & $\begin{array}{l}\text { Group I } \\
\text { ERAS programme } \\
(\mathrm{n}=13)\end{array}$ & $\begin{array}{l}\text { Group II } \\
\text { Traditional care } \\
(\mathrm{n}=13)\end{array}$ & $\begin{array}{l}P \text {-value } \\
\text { Primary outcome }\end{array}$ \\
$\begin{array}{ll}\text { Total LOS (days) } \\
\text { Secondary outcomes }\end{array}$ & $5.0[3-10]$ & $7.0[3-12]$ & $0.305^{\mathrm{b}}$ \\
$\begin{array}{l}\text { Functional Recovery (days) } \\
\text { Complications (grade) }\end{array}$ & $3[1-7]$ & $5[2-8]$ & $0.044^{\mathrm{b}}$ \\
$\begin{array}{l}\text { Conversions } \\
\text { Blood loss (ml) }\end{array}$ & $2(\mathrm{I})$ & $2(\mathrm{I})$ & 1.0 \\
Operation time (min.) & 2 & 2 & 1.0 \\
\hline
\end{tabular}

${ }^{\text {a }}$ Continuous variables are expressed as median [range].

P-values by Fisher's exact test, except ${ }^{\mathrm{b}}$ Mann-Whitney $U$ test.

ERAS, Enhanced Recovery After Surgery; LOS, length of stay 
In both groups, functional recovery was achieved a median of 2 days earlier than actual discharge. In the ERAS group, patients met the discharge criteria on median postoperative day 3 (range 1-7 days), compared with the traditional care group, which met the discharge criteria on median postoperative day 5 (range 2-8 days). The difference between the groups in functional recovery proved to be statistically significant $(P<0.044)$, as did the difference in delay in discharge $(P<0.032)$.

In group 1, resected specimens included two hepatocellular adenomas, two focal nodular hyperplasias (FNHs), an angiomyolipoma, a haemangioma, a presumed metastases which proved to be an inflammatory pseudotumour after chemotherapy, an intra-hepatic accessory gallbladder with inflammation, a hepatocellular carcinoma and four metastases of colorectal origin. Resection margins were free of disease in 12 cases; one resected adenoma extended to the resection margin. In group 2, resected specimens included three adenomas, six FNHs and two haemangiomas and, in two patients, three metastases of colorectal origin. Resection margins were free of disease in eight patients, were not reported (benign tumour) in four and involved a haemangioma in the resection surface in one.

No late complications were recorded in group 1 during the follow-up period (median 3 months, range 1-23 months). In the follow-up period (median 6 months, range 1-27months) in group 2, one patient developed colorectal metastases in the lungs and liver (segments VI and VII) and simultaneous acute myeloid leukemia. No palliative chemotherapy was given to this patient because of his clinical condition and he died 12 months postoperatively.

\section{DISCUSSION}

This study investigated the added value of an enhanced recovery programme in laparoscopic liver surgery. To the best of our knowledge, this represents the first such study ever conducted (although it is only a small pilot study). The results suggest that initiation of a multimodal enhanced recovery programme in laparoscopic liver surgery is feasible. It also shows that it is safe to implement an evidence-based enhanced recovery programme, as there were only two minor complications in the ERAS group and two conversions in each group. The difference in median LOS of two days did not attain significance, but this probably reflects the small number of patients in this study. Importantly, the current study provides evidence that an enhanced recovery programme is associated with expedited functional recovery. Patients in the laparoscopic ERAS group fulfilled discharge criteria 2 days earlier than control patients.

Patients in the enhanced recovery group had less blood loss. This may have resulted from the implementation of the ERAS protocol, which prescribes a low CVP and the avoidance of excessive perioperative fluids. However, although this out- 
come is significant, it remains unclear whether it is resulted solely from the implementation of ERAS. This difference in blood loss may also have been clouded by the learning curve effect because the patients operated in group 2 (traditional care) were the first patients to be treated laparoscopically in the participating centres.

Most authors recommended that laparoscopic procedures on liver resections of solid tumours preferably begin with the left lateral segments or anterolateral small lesions of the right side. ${ }^{13,14}$ Tumours of the left lateral side, particularly benign tumours, constitute only a small fraction of the minor resections in most units. This is probably one of the reasons for the small number of patients in this pilot study. It was also considered unethical to prospectively compare effects in an intervention (ERAS) group with effects in a control group recieving traditional care in Maastricht,because the ERAS protocol includes several evidence-based care elements aimed reducing surgical stress and postoperative catabolism. ${ }^{6-8}$

Studies on colorectal laparoscopy and fast-track programmes have shown conflicting results regarding the additional outcomes of minimally invasive surgery performed within a perioperative ERAS programme. In a single-centre study, the laparoscopic approach seemed to improve the outcome in terms of LOS, whereas other studies, including a randomised controlled trial, showed no additional effect of laparoscopy on length of stay. ${ }^{15-17}$ Therefore, it has been suggested that there is a need for further randomised trials to evaluate the additional effect of laparoscopy within fast track recovery programmes in colorectal surgery. ${ }^{5,15,16}$ At present, there is only limited evidence available about the effects on LOS of a multimodal, fast-track setting in liver surgery. ${ }^{4}$

In the current study, a difference between the date of functional recovery of patients undergoing a liver resection and the day of actual discharge was observed, as we previously reported in a colonic ERAS programme. ${ }^{11}$ A significant difference of a median of 2 days in time to full functional recovery was observed between the ERAS-treated group and the traditional care group. It is remarkable that, despite the preoperative counselling in the ERAS programme, a difference between functional recovery and actual day of discharge was still observed. This implies that the difference in LOS may be even more pronounced if patients do actually go home as soon as they are functionally recovered according to predefined criteria. ${ }^{8,11}$ Delay in discharge and the presence of complications were associated with increased LOS. For future studies, functional recovery may be a better criterion with which to investigate differences in outcome of surgical treatments. Establishing the effect of ERAS programmes on outcomes in laparoscopic liver surgery in a more structured manner is a challenge yet to be met.

Acknowledgements. The authors thank the patients who agreed to participate in the study and the surgeons, anaesthetists and nursing colleagues who helped implement the protocol. 
The Enhanced Recovery After Surgery initiative was funded principally by Nutricia Healthcare (Zoetermeer, the Netherlands), which also sponsored preparatory meetings with an unrestricted grant. C.H.C.D. was supported by a grant from the Dutch Organization for Scientific Research (NWO Clinical Fellowship 907-00-033).

\section{REFERENCES}

1. Gagner M, Pomp A, Heniford BT, Pharand D, Lacroix A. Laparoscopic adrenalectomy: lessons learned from 100 consecutive procedures. Ann Surg 1997; 226(3):238-246.

2. Trondsen E, Reiertsen O, Andersen OK, Kjaersgaard P. Laparoscopic and open cholecystectomy. A prospective, randomized study. Eur J Surg 1993; 159(4):217-221.

3. Simillis C, Constantinides VA, Tekkis PP, Darzi A, Lovegrove R, Jiao L et al. Laparoscopic versus open hepatic resections for benign and malignant neoplasms-a meta-analysis. Surgery 2007; 141(2):203-211.

4. van Dam RM, Hendry PO, Coolsen MM, Bemelmans MH, Lassen K, Revhaug A et al. Initial experience with a multimodal enhanced recovery programme in patients undergoing liver resection. $\mathrm{Br} \mathrm{J}$ Surg 2008; 95(8):969-975.

5. Wind J, Polle SW, Fung Kon Jin PH, Dejong CH, von Meyenfeldt MF, Ubbink DT et al. Systematic review of enhanced recovery programmes in colonic surgery. Br J Surg 2006; 93(7):800-809.

6. Fearon KC, Ljungqvist $\mathrm{O}$, Von Meyenfeldt $\mathrm{M}$, Revhaug $\mathrm{A}$, Dejong $\mathrm{CH}$, Lassen $\mathrm{K}$ et al. Enhanced recovery after surgery: a consensus review of clinical care for patients undergoing colonic resection. Clin Nutr 2005; 24(3):466-477.

7. Kehlet H. Manipulation of the metabolic response in clinical practice. World J Surg 2000; 24(6):690695.

8. Maessen J, Dejong CH, Hausel J, Nygren J, Lassen K, Andersen J et al. A protocol is not enough to implement an enhanced recovery programme for colorectal resection. Br J Surg 2007; 94(2):224-231.

9. Podore PC, Throop EB. Infrarenal aortic surgery with a 3-day hospital stay: A report on success with a clinical pathway. J Vasc Surg 1999; 29(5):787-792.

10. Trondsen E, Mjaland O, Raeder J, Buanes T. Day-case laparoscopic fundoplication for gastrooesophageal reflux disease. Br J Surg 2000; 87(12):1708-1711.

11. Maessen JM, Dejong CH, Kessels AG, von Meyenfeldt MF. Length of Stay: An Inappropriate Readout of the Success of Enhanced Recovery Programmes. World J Surg 2008; 32(6):971-975.

12. Clavien PA, Sanabria JR, Strasberg SM. Proposed classification of complications of surgery with examples of utility in cholecystectomy. Surgery 1992; 111(5):518-526.

13. Cherqui D, Husson E, Hammoud R, Malassagne B, Stephan F, Bensaid S et al. Laparoscopic liver resections: a feasibility study in 30 patients. Ann Surg 2000; 232(6):753-762.

14. Gigot JF, Glineur D, Santiago AJ, Goergen M, Ceuterick M, Morino M et al. Laparoscopic liver resection for malignant liver tumors: preliminary results of a multicentre European study. Ann Surg 2002; 236(1):90-97.

15. Basse L, Jakobsen DH, Bardram L, Billesbolle P, Lund C, Mogensen T et al. Functional recovery after open versus laparoscopic colonic resection: a randomized, blinded study. Ann Surg 2005; 241(3):416-423.

16. MacKay G, Ihedioha U, McConnachie A, Serpell M, Molloy RG, O'Dwyer PJ. Laparoscopic colonic resection in fast-track patients does not enhance short-term recovery after elective surgery. Colorectal Dis 2007; 9(4):368-372.

17. Raue W, Haase O, Junghans T, Scharfenberg M, Muller JM, Schwenk W. 'Fast-track' multimodal rehabilitation programme improves outcome after laparoscopic sigmoidectomy: a controlled prospective evaluation. Surg Endosc 2004; 18(10):1463-1468. 


\section{Chapter 11}

\section{Laparoscopic liver resection in the Netherlands: How far are we?}

J.H.M.B. Stoot, E.M. Wong-Lun-Hing, I. Limantoro, R. Visschers, O.R.C. Busch, R. Van Hillegersberg, K.M. De Jong, A.M. Rijken, G.Kazemier, S.W.M. Olde Damink, T.M. Lodewick, M.H.A. Bemelmans, R.M. van Dam, C.H.C. Dejong On behalf of the Dutch Liver Collaborative Group.

Adapted from Dig Surg 2012;29:70-78

Preliminary results of this study were presented to the Congress of the European Hepato-Pancreatico Biliary Association in Athens, Greece, June 2009, to the $17^{\text {th }}$ international Congress of the European Association for Endoscopic Surgery in Prague, Tsjech Republic in June 2009, to the Annual congress of the Association of Surgeons of Great Britain and Ireland in Glasgow, Scotland in May 2009 and to the European Postgraduate Gastro-Intestinal Surgical School during "One day on techniques of liver resection" at the AMC in Amsterdam, the Netherlands in March 2011. 


\section{ABSTRACT}

Background: The objective of this study was to provide a systematic review on the introduction of laparoscopic liver surgery in the Netherlands, to investigate the initial experience with laparoscopic liver resections and to report on the current status of laparoscopic liver surgery in the Netherlands.

Methods: A systematic literature search of laparoscopic liver resections in the Netherlands was conducted using PubMed/MEDLINE. Analysis of initial experience with laparoscopic liver surgery was performed by case-control comparison of patients undergoing laparoscopic left lateral sectionectomy matched with patients undergoing the open procedure in the Netherlands between the years 2000 to 2008. Furthermore, a nationwide survey was conducted in 2011 on the current status of laparoscopic liver surgery.

Results: The systemic review revealed only 6 Dutch reports on actual laparoscopic liver surgery. Matched case-control comparison showed significant differences in length of hospital stay, blood loss and operation time. Complications did not differ significantly between the two groups (26\% vs. 21\%). The 2011 survey showed that 21 centres in the Netherlands performed formal liver resections and that $49(=5 \%$ of total) laparoscopic liver resections were performed in 2010.

Conclusion: The systematic review revealed that very few laparoscopic livers were performed in the Netherlands in the previous millennium. The matched casecontrol comparison of laparoscopic and open left lateral resection showed reduction in hospital length of stay with comparable morbidity. The laparoscopic technique has been slowly adopted in the Netherlands, but its popularity seems to increase in recent years. 


\section{INTRODUCTION}

In the past two decades, the popularity of laparoscopic surgery has grown. The introduction of laparoscopic cholecystectomy ${ }^{1,2}$ has led to an increasing number of indications for this technique and has encouraged surgeons to develop a laparoscopic approach for many procedures including liver resections. ${ }^{3}$ Laparoscopic liver resections are technically demanding and thought to be time-consuming ${ }^{4,5}$ but may have several important benefits for the patient compared to open liver surgery. ${ }^{6-8}$ The putative advantages of this minimally invasive procedure include reduced blood loss, ${ }^{6,7}$ less postoperative pain, ${ }^{5,9,10}$ earlier recovery and shorter postoperative hospital stay, ${ }^{5,7-12}$ and improved cosmetic aspects. ${ }^{9,12}$ Reoperations are reported to be easier due to reduced adhesions. ${ }^{9-12}$ Also, open-close procedures with large incisions can be avoided when peritoneal metastases are detected at laparoscopy.

In the Netherlands, the gold standard for resection of liver tumours still is the open approach. There is no best level of evidence (level 1) to underpin the supposed major benefits of the laparoscopic approach, but the latter has been proven to be feasible and safe in many international expert centres. A recent consensus statement on laparoscopic liver resections states that resection of segments 2 and 3 by the laparoscopic approach should be the standard if experience is available. ${ }^{13}$ In addition, the largest reviews hitherto conducted concluded that laparoscopic liver surgery is feasible and safe in minor as well as major liver resections when performed in experienced centres. ${ }^{14,15}$ However, randomised trials comparing the open and laparoscopic technique for liver resections are still lacking.

The purpose of our study was (1) to provide a historical overview on the introduction and initial experience of laparoscopic liver surgery in the Netherlands against the background of the latest international developments, (2) to report on the present state of laparoscopic liver surgery in the Netherlands, and (3) to provide insight into current and future developments of the minimally invasive liver resection technique within the Netherlands. To this purpose, a systematic review of all Dutch papers reporting on laparoscopic liver surgery was done and key leaders were informally approached. A matched case-control comparison was carried out of laparoscopic versus open liver resections in the Netherlands performed from 2000 to 2008. Finally, recent advances made in the Dutch surgical field of laparoscopic hepatectomies were investigated by means of a nationwide survey. 


\section{METHODS}

\section{Systematic review of laparoscopic liver surgery in the Netherlands - Past Experience}

An online search was performed in PubMed / Embase databases for reports of Dutch groups on their experience with laparoscopic liver surgery in humans. Databases were searched from the earliest data available until 1 June 2011 on a combination of the following search keywords: 'laparoscopy', 'liver surgery', 'hepatic surgery', 'hepatic resection', 'hepatectomy', 'minimally invasive liver surgery', 'minimally invasive hepatic resection', 'Dutch', 'the Netherlands'. Biliary tumours were excluded. Titles and abstracts were screened (E.W.L.H.) and relevant articles were selected. The full texts of eligible papers were retrieved to provide a historical overview. The reference lists of retrieved articles were reviewed for additional potentially relevant studies. To complete the historical picture, key leaders in gastrointestinal surgery were informally queried about their past experience and recollection of the evolution of laparoscopic liver surgery in the Netherlands.

\section{Initial experience with laparoscopic liver resections and case-control comparison - Present State}

\section{Patients}

All major liver centres in the Netherlands were contacted during 2008 by phone and/or e-mail and asked to participate in this study. Of the fourteen major HPB centres in the Netherlands, only seven had performed laparoscopic liver resections. Six of these centres agreed to collaborate. Data were obtained retrospectively from six separate prospectively collected databases used in these six HBP units and were pooled for further analysis. All patients who underwent laparoscopic left lateral sectionectomies of the liver in the six centres between 2000 and 2008 were included in this multicentre study. The laparoscopic group of patients was compared in a case-matched comparison approach with a group of patients undergoing the same type of liver resection as an open procedure in that era in the participating 6 centres in a proportion of 1:3. This approach, and the choice to only include left lateral sectionectomies, was done to eliminate bias as much as possible and increase the external validity of the conclusions. The investigators (J.H.M.B.S and I.L.) were unaware of the primary outcome or secondary outcomes during the selection process. Patients undergoing left lateral resections in combination with colonic resections were excluded.

In each unit, patients were preoperatively discussed in a multidisciplinary meeting, after evaluation of liver function tests and radiologic liver workup includ- 
ing abdominal ultrasonography, CT, MRI and/or CT-PET scan. Patients with benign as well as malignant solid liver lesions were included in this study. Patients with cystic lesions of the liver were excluded. Laparoscopic liver resection was considered if the lesion was located in the left lateral segments (segment 2 and 3 according to Couinaud's classification). The choice to perform either a laparoscopic or open procedure was at the discretion of the attending surgeon in consent with the patient. Preoperative information consisted of medical history, preoperative diagnosis and American Society of Anaesthesiologists (ASA) classification.

\section{Surgical procedures}

The open procedure was started with a $15-$ to $25-\mathrm{cm}$ incision according to the preference of the surgeon. During operation the left liver was mobilised and central venous pressure was maintained at $2-6 \mathrm{mmHg}$. Transection of the liver was performed according to the preference of the surgeon: the Cavitron Ultrasonic Surgical Aspirator (CUSA ${ }^{\circledR}$; Valleylab, Boulder, Colorado, USA) and argon beam coagulator (Bircher Ind., Ltd., Englewood, Colo.,USA) with or without the use of Pringle's manoeuvre, Ultracision Harmonic ACE (Ethicon Endosurgery, Johnson \& Johnson, USA) or Ligasure (Covidien, USA). Only occasionally was the clamp-crush technique used. Minor crossing vessels and biliary radicals were divided using polypropylene sutures or clips. The portal pedicles and hepatic veins were divided and ligated with a running polypropylene suture. In some procedures, vascular staplers such as e.g. Autosuture EndoGIA (Covidien) were used. After removal of the liver specimen, the raw surface of the liver remnant was subjected to argon beam coagulation and sealed with TachoSil ${ }^{\circledR}$ (Nycomed, Zurich, Switzerland) or Tissuecoll (Baxter, Vienna, Austria) if considered appropriate.

The laparoscopic procedure was performed as described in detail previously. ${ }^{16}$ The patient was in the supine French position and abdominal access was achieved by transumbilical open insertion of a laparoscope. Pneumoperitoneum was kept at 10-14 mmHg; three or four additional 12-mm trocars were added and a $30^{\circ}$ laparoscope was used routinely. The central venous pressure was maintained at 2-6 $\mathrm{mmHg}$. Hepatic transection of parenchyma and minor crossing vessels and biliary radicals was mainly performed with harmonic scalpel (Ultracision, Ethicon Endosurgery, Johnson \& Johnson, USA) or the Ligasure (Covidien, USA). The segmental portal pedicles and left hepatic vein were stapled using a vascular stapler (EndoGIA Autosuture, Covidien). Resected specimens were placed in a plastic bag (Endocatch Autosuture, Covidien) and removed through a separate incision.

\section{Parameters and outcome}

Peri- and postoperative parameters included type of resection, operation time, blood loss, need for transfusion, conversion of laparoscopic to open procedure, postoperative complications, postoperative length of hospital stay and pathologic 
assessment of the resected liver segment. Blood loss was measured by the suction device and the weight of the gauzes and recorded in operation notes and/or anaesthesia reports. In case of discrepancy the highest amount was used. Data on surgical technique consisted of information about type of incisions, type of liver resection, method of liver transection, haemorrhage control, use of haemostatic agents, Pringle-manoeuvre, and method of extraction of surgical specimen. In one centre (Maastricht University Medical Centre: MUMC), a multimodal perioperative enhanced recovery programme $\left(\right.$ ERAS $\left.^{\circledR}\right)$ was followed for all patients undergoing liver surgery. ${ }^{16,17}$ This was also recorded as a parameter. Criteria for discharge in the ERAS setting were described previously. ${ }^{16,17}$ Discharge in the traditional setting was at the discretion of the attending surgeon.

Primary outcome of the study was hospital length of stay. Secondary outcome measures comprised complications (including mortality and conversion rates), duration of operation and blood loss. Postoperative complications were defined according to the intenational grading system of Dindo et $a l^{18}$

\section{Current status of laparoscopic liver surgery - Future Developments}

To get an indication of the volumes currently being operated on in Dutch hospitals, and to see whether they had increased in the years following the introduction of the minimally invasive technique, we approached all Dutch hospitals that indicated to perform liver surgery in January 2011 by e-mail. This contained three questions surveying on (1) whether the hospital performed formal liver resections (not only deroofing of cysts), (2) how many open / laparoscopic major or minor hepatic resections were performed in 2010 and (3) whether the provided data were real or estimated numbers. Centres that did not reply on the request to complete the survey were approached by phone in February 2011.

\section{Statistical analysis}

Data were analysed by J.S., I.L. and R.V, if necessary an independent statistician was consulted. Continuous variables are expressed as mean $( \pm$ standard error of the mean: SEM). Data were analysed according to the intention to treat principle. Univariable analysis was performed using Pearson's $\chi^{2}$ test (or Fisher's exact test where appropriate) to investigate differences between open and laparoscopic procedures regarding sex, indication, pathology, resection margins, ERAS, use of Pringle manoeuvre, method of liver transection, haemorrhage control and haemostatic agents, use of staplers and complications. Mann-Whitney $U$ test was used for univariable analysis to investigate differences between open and laparoscopic surgery regarding age, ASA classification, tumour diameter, type of incision, complication classification, hospital length of stay (days), duration of operation ( $\mathrm{min}$ ), blood 
loss $(\mathrm{ml})$ and number of transfusions. The relation between patient characteristics and operative strategy, and length of hospital stay was analyzed with univariable linear regression analysis. All independent variables with a two-tailed $\mathrm{p}$ value below 0.200 were included into a multivariable linear regression model using backward analysis to assess which parameters were significantly and independently related with length of hospital stay. Also, the group effect of open versus laparoscopic resections on post-operative haemoglobin was analysed using multivariable linear regression analysis correcting for preoperative haemoglobin levels. A p value below 0.05 was considered to indicate statistical significance. Statistical analyses were performed using SPSS software (version 15; SPSS Inc., USA).

\section{RESULTS}

\section{Systematic review of laparoscopic liver surgery in the Netherlands - Past Experience}

A total of 91 abstracts were retrieved that met the search terms and after review 19 abstracts remained eligible. Of these 19 articles, 6 reported on actual laparoscopic liver surgery, 12 described the use of laparoscopy and /or laparoscopic ultrasound for staging in patients with hepatic tumours, and one article provided a literature review on liver resection and laparoscopy (Fig. 1). The informal interviews and articles eligible for review revealed that the first two laparoscopic liver resection were performed in Amsterdam by the group of Cuesta et al. in $1995 .{ }^{19}$ In the following years only one article from the same group was published reporting on a series of laparoscopically operated patients $(n=10)$. Seven underwent a minor laparoscopic resection and three underwent fenestration of hepatic cysts. ${ }^{20}$ After these initial reports on minimally invasive liver surgery three articles were published with regard to laparoscopic treatment of polycystic liver disease. ${ }^{21-23}$ Later series of 26 laparoscopic liver resections provided evidence that this procedure could be performed safely in the Netherlands. ${ }^{16}$ 


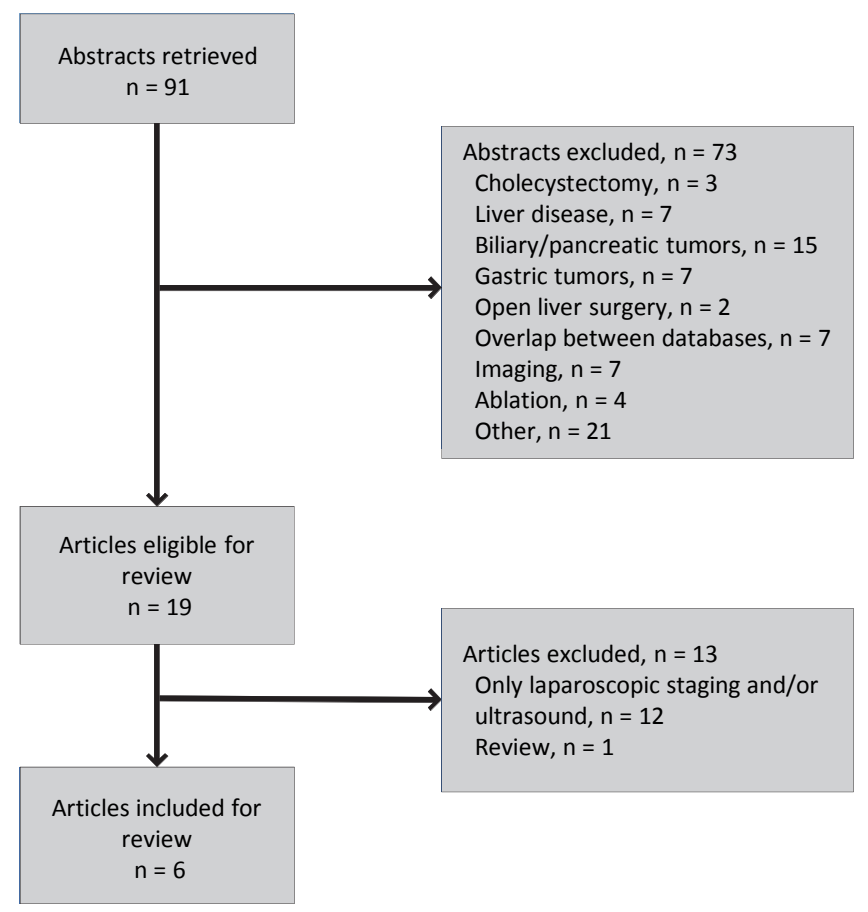

Figure 1. Selection of studies eligible for systematic review.

Initial experience with laparoscopic liver resections and case-control comparison - Present State

\section{Patient's characteristics}

Between 2000 and 2008, the laparoscopic approach for hepatic left lateral sectionectomy was performed in thirty patients in 6 centres in the Netherlands (mean age $50 \pm 3$ years) and completed laparoscopically in 27 patients. Five of the laparoscopic liver resections were performed hand-assisted. In the open group, 90 patients were matched for the same type of resection (mean age $52 \pm 2$ years). Demographic data of both groups are reported in table 1 . There were no significant differences in age, ASA classification, indication, resection margins and tumour size. 
Table 1. Clinical and pathological features of the case-control study

\begin{tabular}{|c|c|c|c|}
\hline & $\begin{array}{l}\text { Laparoscopic Group } \\
(\mathrm{n}=30)\end{array}$ & $\begin{array}{l}\text { Open Group } \\
(n=90)\end{array}$ & $\mathrm{p}$-value \\
\hline$\overline{\text { Sex }\left(\text { male } / \text { female) }{ }^{a}\right.}$ & $5 / 25$ & $35 / 55$ & 0.043 \\
\hline Age (years) ${ }^{b}$ & $50(3)$ & $52(2)$ & 0.584 \\
\hline ASA $1^{\mathrm{b}, \mathrm{c}}$ & 10 & 18 & 0.625 \\
\hline ASA 2 & 16 & 38 & \\
\hline ASA 3 & 2 & 16 & \\
\hline ASA 4 & 0 & 1 & \\
\hline Indication (malignant/benign) ${ }^{a}$ & $11 / 19$ & $39 / 51$ & 0.091 \\
\hline Tumour size, $\mathrm{cm}^{\mathrm{b}}$ & $4.8 \pm 0.5$ & $5.3 \pm 0.4$ & 0.673 \\
\hline Pathology (malignant/benign) ${ }^{a, c}$ & $7 / 22$ & $48 / 42$ & 0.007 \\
\hline Resection margins (R0/R1/R2) ${ }^{\mathrm{a}, \mathrm{c}, \mathrm{d}}$ & $19 / 1 / 1$ & $64 / 8 / 2$ & 0.597 \\
\hline ERAS (yes/no) ${ }^{a}$ & $11 / 19$ & $8 / 92$ & 0.001 \\
\hline
\end{tabular}

Values for tumour size are expressed as mean \pm standard error of mean.

a Pearson's Chi square test (or Fisher exact test where appropriate).

${ }^{b}$ Mann Whitney U-test.

${ }^{\mathrm{c}}$ Results do not add up to total values as a result of missing data.

${ }^{d}$ In the laparoscopic group, two (R1 and R2) resection margins were positive, both in patients with benign disease (adenoma and haemangioma, respectively). In the open control group, the R2 resections were suspected benign lesions preoperatively. However, one of these $\mathrm{R} 2$ resections proved to be malignant (hepatocellular carcinoma). Of the R1 resection margins, $3 / 8$ were malignant tumours.

\section{Surgical procedures and parameters}

The Pringle manoeuvre was only used during open surgery (table 2). The method of liver transection in the open group was different from the approach in the laparoscopic group. Mostly, the CUSA and/or argon beam was used in the open group for liver transection. 
Table 2. Operative details on transection and haemostasis techniques and use of devices

\begin{tabular}{|c|c|c|}
\hline & $\begin{array}{l}\text { Laparoscopic Group } \\
(\mathrm{n}=30)\end{array}$ & $\begin{array}{l}\text { Open Group } \\
(n=90)\end{array}$ \\
\hline Use of Pringle manoeuvre & $1(3)^{a}$ & $20(22)$ \\
\hline \multicolumn{3}{|l|}{ Liver transection } \\
\hline Cusa +/- argonbeam & $3(10)$ & $55(61)$ \\
\hline Ultracision & $21(70)$ & $4(4)$ \\
\hline Ligasure & $4(13)$ & $7(8)$ \\
\hline Hydrojet & & $2(2)$ \\
\hline Cusa + ligasure & & $8(9)$ \\
\hline Diathermia +/- endogia & & $4(4)$ \\
\hline Kelly clamp & & $1(1)$ \\
\hline Endogia & $2(7)$ & $4(4)$ \\
\hline \multicolumn{3}{|l|}{ Haemorrhage control $^{\text {b }}$} \\
\hline Diathermia+/- argonbeam & $6(20)$ & $4(4)$ \\
\hline Clips & $6(20)$ & $15(17)$ \\
\hline Prolene & $4(13)$ & $23(26)$ \\
\hline Clips and prolene & $1(3)$ & $39(43)$ \\
\hline Other: use of device & $9(30)$ & $3(3)$ \\
\hline Use of staplers (yes/no) ${ }^{\mathrm{b}}$ & $22 / 3^{a}$ & $19 / 68$ \\
\hline \multicolumn{3}{|l|}{ Haemostatic agents $^{b}$} \\
\hline None & $19(63)$ & $39(43)$ \\
\hline Tachosyl & $4(13)$ & $17(19)$ \\
\hline Tissuecoll & $4(13)$ & $25(28)$ \\
\hline Surgicell & $1(3)$ & $1(1)$ \\
\hline Unknown & $1(3)$ & $7(8)$ \\
\hline
\end{tabular}

Figures in parentheses indicate percentages.

${ }^{a}$ Following conversion to open.

${ }^{\mathrm{b}}$ Results do not add up to total values as a result of missing (non reported) data.

\section{Outcome parameters}

Mean hospital length of stay was $6.0( \pm 0.4)$ days in the laparoscopic group versus $8.1( \pm 0.4)$ days in the open group $(p<0.001)$. Complication rates did not differ significantly between the laparoscopic and open group ( $26 \%$ vs. $21 \%$ ) neither did they differ significantly when complications were graded according to severity intervention score. Three laparoscopic procedures $(10 \%)$ were converted to an open procedure (table 3). Reasons for conversion where either haemorrhage, an additional lesion in segment IV or its proximity to the left hepatic vein. There were no deaths in the laparoscopic group. In the open group, one of the 90 patients (1.1\%) died due to multiple organ failure after sepsis. 
Table 3. Primary and secondary outcomes (mean \pm SEM)

\begin{tabular}{|c|c|c|c|}
\hline & $\begin{array}{l}\text { Laparoscopic Group } \\
(\mathrm{n}=30)\end{array}$ & $\begin{array}{l}\text { Open Group } \\
(n=90)\end{array}$ & p-value \\
\hline Length of stay, days ${ }^{a}$ & $6.0 \pm 0.4$ & $8.1 \pm 0.4$ & $<0.001$ \\
\hline Complications $^{\mathrm{b}}$ & $5(26)$ & $19(21)$ & 0.620 \\
\hline Complications $^{\mathrm{a}}$ & & & 0.832 \\
\hline Grade 1 & $4(13)$ & $8(9)$ & \\
\hline Grade 2 & & $4(4)$ & \\
\hline Grade 3a & & $3(3)$ & \\
\hline Grade $3 b$ & $1(3)$ & & \\
\hline Grade 4a & & $2(2)$ & \\
\hline Grade 4b & & $1(1)$ & \\
\hline Grade 5 & & $1(1)$ & \\
\hline Conversions & $3(10)$ & & \\
\hline Reoperation $^{\text {b }}$ & $1(3)$ & $3(3)$ & 0.589 \\
\hline Blood loss, $\mathrm{ml}^{\mathrm{a}}$ & 288 (99) & $608(97)$ & $<0.001$ \\
\hline Operation time, $\min ^{a}$ & $160(13)$ & $231(11)$ & $<0.001$ \\
\hline
\end{tabular}

Figures in parentheses indicate percentages.

${ }^{a}$ Mann Whitney $U$ test.

${ }^{b}$ Pearson's $\chi^{2}$ test (or Fisher exact test where appropriate).

There was significantly less blood loss in the laparoscopic group compared to the open group ( $p<0.001$; table 3 ). When corrected for preoperative haemoglobin level, multivariable analysis showed that open resection was significantly related with a decrease in postoperative haemoglobin levels compared to the laparoscopic group ( $\mathrm{OR}=-0.520, \mathrm{Cl}-1.022$ to $-0.18, \mathrm{p}=0.043$ ). None of the patients in the laparoscopic group needed a blood transfusion postoperatively in contrast to 22 blood transfusions in the open group. However, this difference was not significant. The mean duration of operation in the laparoscopic group was significantly shorter than in the open group ( $p<0.001$; table 3$)$.

Univariable regression analyses showed that several variables were related with differences in length of hospital stay (table 4). However, after multivariable regression analysis only ASA classification ( $\mathrm{OR}=1.598, \mathrm{Cl} 0.738$ to $2.458, \mathrm{p}<0.001)$, complication grade (OR=1.680, $\mathrm{Cl} 1.124$ to $2.235, \mathrm{p}<0.001)$ and ERAS $(\mathrm{OR}=-2.502, \mathrm{Cl}$ -4.032 to $-0.972, p=0.002$ ) were independently and significantly related with length of hospital stay. 
Chapter 11

Table 4. Univariable linear regression analysis of length of hospital stay

\begin{tabular}{|c|c|c|c|}
\hline Variable & Odds ratio & $95 \%$ confidence interval & P-value \\
\hline Age (years) & 0.040 & 0.001 to 0.079 & 0.047 \\
\hline Sex (male/female) & 1.374 & 0.022 to 2.276 & 0.046 \\
\hline Group (open/laparoscopic) & 2.012 & 0.571 to 3.452 & 0.007 \\
\hline Indication (malignant/benign) & 1.419 & 0.154 to 2.684 & 0.028 \\
\hline Pathology (malignant/benign) & 1.762 & 0.501 to 3.023 & 0.007 \\
\hline Tumour diameter (cm) & 0.091 & -0.129 to 0.311 & 0.413 \\
\hline Complication $^{a}$ & 1.559 & 1.025 to 2.093 & $<0.001$ \\
\hline Duration (minutes) & 0.008 & 0.001 to 0.015 & 0.020 \\
\hline ERAS (yes/no) & -1.392 & -3.136 to 0.352 & 0.117 \\
\hline Reoperation & 0.961 & -4.092 to 6.014 & 0.707 \\
\hline $\mathrm{ASA}^{\mathrm{b}}$ & 1.840 & 0.849 to 2.830 & $<0.001$ \\
\hline Stapler (yes/no) & -1.637 & -3.014 to -0.261 & 0.020 \\
\hline Pringle (yes/no) & -0.762 & -2.130 to 0.606 & 0.272 \\
\hline \multicolumn{4}{|l|}{ Transection } \\
\hline CUSA & 1.523 & -1.803 to 4.848 & 0.366 \\
\hline Ultracision & 0.120 & -3.373 to 3.613 & 0.946 \\
\hline Ligasure & 0.309 & -3.536 to 4.155 & 0.874 \\
\hline Hydrojet & 1.400 & -4.565 to 7.365 & 0.643 \\
\hline CUSA and ligasure & 1.525 & -2.540 to 5.590 & 0.459 \\
\hline Other & -0.350 & -5.133 to 4.433 & 0.885 \\
\hline Kelly & 1.400 & -6.410 to 9.210 & 0.723 \\
\hline Endogia & 0.567 & -3.751 to 4.884 & 0.795 \\
\hline \multicolumn{4}{|l|}{ Haemorrhage control } \\
\hline Diathermia & -1.992 & -4.545 to 0.560 & 0.125 \\
\hline Clips & -0.942 & -3.320 to 1.435 & 0.433 \\
\hline Prolene & 1.197 & -0.606 to 3.000 & 0.191 \\
\hline Other & -1.276 & -3.653 to 1.102 & 0.289 \\
\hline \multicolumn{4}{|l|}{ Haemostatic agents } \\
\hline Tachosyl & -0.024 & -5.387 to 5.340 & 0.993 \\
\hline Tissuecol & 0.121 & -5.178 to 5.419 & 0.964 \\
\hline Surgicell & 3.000 & -4.248 to 10.248 & 0.414 \\
\hline None & -0.114 & -5.328 to 5.100 & 0.965 \\
\hline
\end{tabular}

${ }^{a}$ Complication according to Dindo et al. ${ }^{18}$; increase per unit increase severity.

${ }^{\mathrm{b}}$ Increase per unit increase severity.

\section{Current status of laparoscopic liver surgery - Future Developments}

The response rate to the nationwide survey was $81 \%$; 30 out of 37 approached hospitals responded. The seven non-responding centres were all district general hospitals centres, except one university medical centre. In total 21 centres performed formal liver resections in 2010, the remaining 9 centres only performed 
deroofing procedures. The total number of hepatic resections performed in 2010 by the responding centres in the Netherlands was 966 . This total number of resections consisted of 444 major and 522 minor hepatectomies (Fig. 2). During 2010 all Dutch hospitals together performed only 49 laparoscopic liver resections; $5 \%$ of all resections (Fig. 3). All these minimally invasive surgical procedures were reported to be minor hepatic resections.

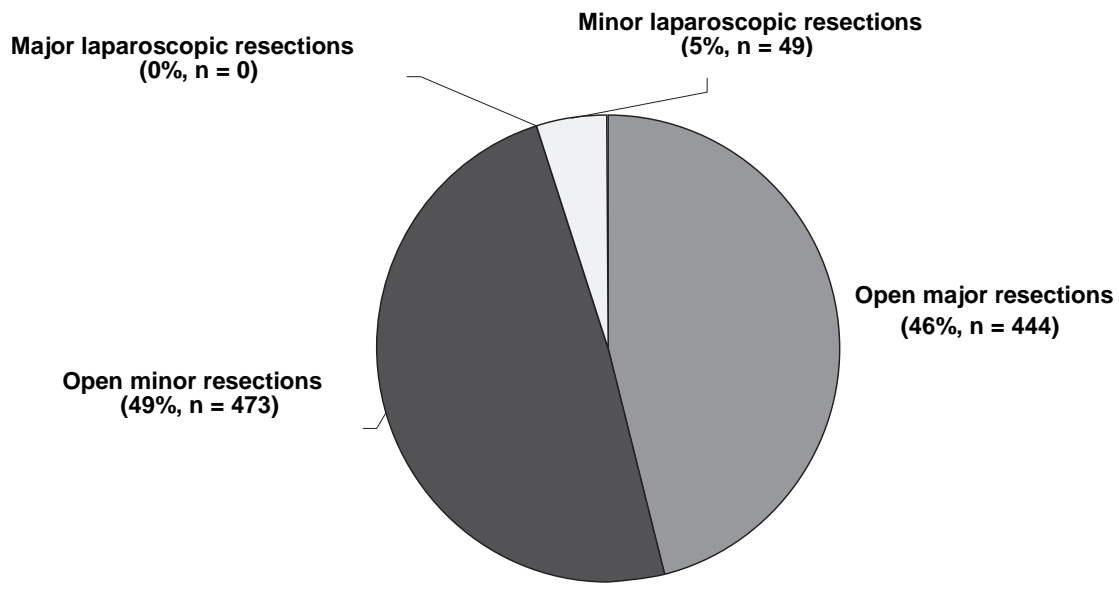

Figure 2. Results of a nation wide survey with actual numbers of laparoscopic and open liver resections performed during 2010 in the Netherlands.

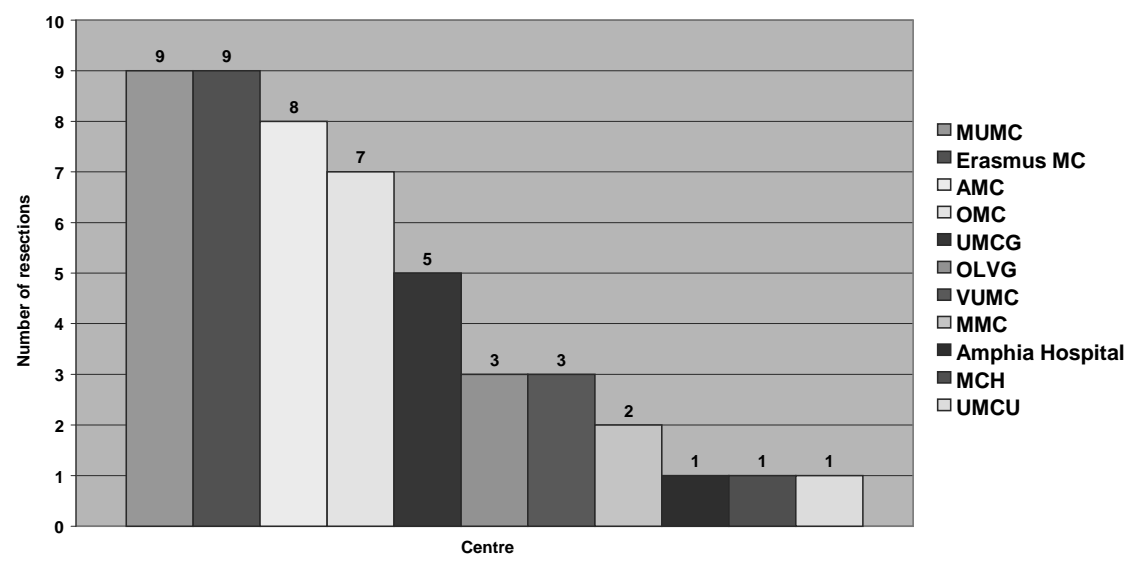

Figure 3. Results of a nationwide survey of total laparoscopic (minor) liver resections performed during 2010 in the Netherlands per centre. 


\section{DISCUSSION}

This study provides a historical overview and reports on the initial experience and the current status of laparoscopic liver surgery in the Netherlands. A systematic review of the literature showed that the laparoscopic approach for hepatic resection was introduced in the Netherlands during the 1990s and that some, but limited experience has been gained. It is clear from the data and from additional information obtained by interviewing key leaders that only very few laparoscopic livers were performed in the previous millennium.

Compared to countries that have traditionally always played a pioneering role in (laparoscopic) liver surgery, such as France, the Netherlands have fallen behind with regard to the implementation of the minimally invasive technique. Many surgeons considered an open partial liver resection to be major surgery, associated with significant mortality and morbidity. Dutch surgeons remained reluctant to perform this type of surgery. ${ }^{24}$ Illustrating this fact is that only $110-130$ partial liver resections (including benign tumours) were performed between 1984 and 1987. At the end of the 1980s and 1990s several series on the experience with open hepatic resection were published by different Dutch centres. ${ }^{25,26}$

The laparoscopic technique for liver resections was introduced in the Netherlands during the 1990s for diagnostic laparoscopies and liver biopsies. Later the indications were extended to fenestration of liver cysts and anatomic liver resections. ${ }^{27-32}$ The group of Cuesta et al. ${ }^{19}$ was in 1995 the first to report two cases of limited laparoscopic liver surgery of segment II and IV in the Netherlands. A few years later, in 2001, a small retrospective series $(n=10)$ was published that demonstrated encouraging results concerning operative blood loss, postoperative complications and hospital length of stay after wedge and left lateral hepatectomy. Furthermore, they concluded that laparoscopic treatment should be considered in selected patients with benign and malignant lesions in the left lobe or frontal segments of the liver. ${ }^{20}$

While the first limited laparoscopic liver resection in the Netherlands was performed in 1995, the first laparoscopic liver resection worldwide, a wedge resection, had already been performed by Gagner's group in 1992. Four years thereafter, Azagra's group reported the first anatomic liver resection. ${ }^{3}$ The following few years the minimally invasive technique was further developed and adopted, resulting in the first laparoscopic left lateral sectionectomy for living liver donation ${ }^{33}$ and the first reports on robotic liver surgery ${ }^{34,35}$ Initially, laparoscopic liver resection was challenging because of the difficulties concerning safe mobilization and exposure of this fragile and heavy organ. ${ }^{3,36,37}$ Small, superficial and peripheral lesions in anterolateral segments were considered most amenable to laparoscopic liver resection. Surgeons with extensive experience in laparoscopy and hepatic surgery have performed laparoscopic major hepatic resections with satisfactory outcomes. ${ }^{28,38,39}$ 
Recent large reviews by Reddy et al. ${ }^{15}$ and Nguyen et al. ${ }^{14}$ show favourable outcomes after laparoscopic resection. ${ }^{14,15}$ Patient benefits included less operative blood loss ${ }^{6,7}$ less postoperative pain ${ }^{5,9,10}$ and narcotic requirement, improved cosmetic aspects, ${ }^{9,12}$ and a shorter length of hospital stay ${ }^{5,7-12}$ with postoperative morbidity and mortality comparable to open liver resection. In addition, the minimally invasive approach seems to be cost-effective. ${ }^{40,41}$ Potential limitations and disadvantages of laparoscopic liver resection include a significant learning curve, potential bleeding which may be more difficult to control laparoscopically, inadequate assessment of the liver for additional lesions, and increased risk for gas embolism. ${ }^{15,42}$ Air embolism may occur when high-pressure pneumoperitoneum is used. 36, ${ }^{43}$ However, gas embolism is rare and usually well tolerated. ${ }^{5}$ Other concerns have been raised about the potential dissemination of malignant cells during laparoscopic resection. ${ }^{44-46}$ Some authors have suggested that tumour dissemination does not increase by laparoscopy, ${ }^{47-49}$ and recent reports regarding this subject show long term survival rates comparable with open surgery. ${ }^{12,37,50,51}$

At present, after more than 10 years of experience and following advances in laparoscopic technology, the surgical community has accepted that laparoscopic liver surgery is feasible and safe. ${ }^{3,7,8,10,51-53}$ This is especially true for resection of left lateral segments and right anterior segments. 4, 9, 11, 54 Some of the advantages reported in literature were also found in the present study. The present multicentre case-control study on laparoscopic left lateral sectionectomies in the Netherlands between 2000 and 2008 demonstrated that length of hospital stay for the laparoscopic approach for left lateral sectionectomies was reduced by 2 days. Furthermore, the laparoscopic approach resulted in faster procedures with reduced blood loss, no occurrence of gas embolisms and comparable morbidity. In a multivariable analysis, length of hospital stay appeared to be related with ASA classification, complications and ERAS.

Left lateral sectionectomy currently is the most common laparoscopic liver resection for solid tumours in the Netherlands. However, only a minority of left lateral sectionectomies were performed laparoscopically between 2000 and 2008 . The laparoscopic approach has gained gradual acceptance by Dutch surgeons, but is still not standard. A structured implementation may allow the Dutch surgical community to catch up with international developments. Recently, van Dam and Topal performed the first major laparoscopic liver resection in the Netherlands (anatomical right hemihepatectomy, Maastricht 2011).

The question remains why the laparoscopic left sectionectomy in this study proved to be faster than the open counterpart. Differences in technique in transection of the liver parenchyma and the vascular and biliary structures may have added to a faster transection. Reduced blood loss, either caused by different transection devices or pneumoperitoneum, may reduce the need for prolene stitches and 
haemostatic clips, which can be time consuming. Lastly, there is no need to close the abdomen after the procedure.

The survey results demonstrate an increase in both totally laparoscopic and totally open liver resections performed in the Netherlands. Although more centres have adopted the laparoscopic approach in recent years, individual centre volumes remain low. Only $5 \%$ of all liver resections were performed laparoscopically, which is significantly less compared to other countries where average percentages of laparoscopic hepatic resections range between 20 and $80 \%{ }^{13}$ If surgeons in the Netherlands aim to increase the portion of laparoscopic liver resections, liver surgery should be further centralized. Furthermore, a central training, proctoring and credentialing infrastructure should be developed to improve quality outcome parameters and to allow units to become (high volume) expert centres.

The foundation of the Dutch Liver Collaborative Group in 2003 gave a new impulse to minimally invasive liver surgery, and is in concordance with recently made recommendations that national and international societies, as well as governing boards, should become involved in the goal of establishing training standards and credentialing. ${ }^{13}$ Goals of this workgroup are to facilitate collaboration of medical centres in the field of hepatic surgery, initiate and facilitate research in liver surgery (especially multicentre trials), facilitate training, education and adoption and to establish a quality control and auditing system for Dutch liver surgery centres

\section{Conclusions}

Minimally invasive liver surgery is gradually being adopted in the Netherlands. The laparoscopic (left sided) liver resection is a safe procedure and it probably results in a shorter hospital length of stay with comparable morbidity. Laparoscopic liver resections appear to be faster. More importantly, ASA classification, complications and ERAS proved to be important prognostic variables for reduced length of hospital stay in Dutch hospitals. In the future, the Dutch Liver Collaborative Group should continue to play an important role in the further adoption and centralization of minimally invasive liver surgery. Conducting an RCT on laparoscopic liver resection may add to the body of evidence supporting broader introduction of minimally invasive liver surgery.

\section{Acknowledgements}

We would like to thank our collegues from the Dutch Liver Surgery Collaborative Group for the kind assistance and cooperation. 


\section{REFERENCES}

1. Reddick EJ, Olsen DO Laparoscopic laser cholecystectomy. A comparison with mini-lap cholecystectomy Surg Endosc 1989: 3; 131-133

2. Dubois F, Berthelot G, Levard H Laparoscopic cholecystectomy: historic perspective and personal experience Surg Laparosc Endosc 1991: 1; 52-57

3. Dagher I, Proske JM, Carloni A, et al. Laparoscopic liver resection: results for 70 patients Surg Endosc 2007: 21; 619-624

4. Descottes B, Glineur D, Lachachi F, et al. Laparoscopic liver resection of benign liver tumors Surg Endosc 2003: 17; 23-30

5. Farges $\mathrm{O}$, Jagot $\mathrm{P}$, Kirstetter $\mathrm{P}$, et al. Prospective assessment of the safety and benefit of laparoscopic liver resections J Hepatobiliary Pancreat Surg 2002: 9; 242-248

6. Morino M, Morra I, Rosso E, et al. Laparoscopic vs open hepatic resection: a comparative study Surg Endosc 2003: 17; 1914-1918

7. Simillis C, Constantinides VA, Tekkis PP, et al. Laparoscopic versus open hepatic resections for benign and malignant neoplasms--a meta-analysis Surgery 2007: 141; 203-211

8. Kaneko H Laparoscopic hepatectomy: indications and outcomes J Hepatobiliary Pancreat Surg 2005: $12 ; 438-443$

9. Cherqui D, Husson E, Hammoud R, et al. Laparoscopic liver resections: a feasibility study in 30 patients Ann Surg 2000: 232; 753-762

10. Buell JF, Thomas MJ, Doty TC, et al. An initial experience and evolution of laparoscopic hepatic resectional surgery Surgery 2004: 136; 804-811

11. Chang S, Laurent A, Tayar C, et al. Laparoscopy as a routine approach for left lateral sectionectomy Br J Surg 2007: 94; 58-63

12. Gigot JF, Glineur D, Santiago Azagra J, et al. Laparoscopic liver resection for malignant liver tumors: preliminary results of a multicentre European study Ann Surg 2002: 236; 90-97

13. Buell JF, Cherqui D, Geller DA, et al. The international position on laparoscopic liver surgery: The Louisville Statement, 2008 Ann Surg 2009: 250; 825-830

14. Nguyen KT, Gamblin TC, Geller DA World review of laparoscopic liver resection-2,804 patients Annals of surgery 2009: 250; 831-841

15. Reddy SK, Tsung A, Geller DA Laparoscopic Liver Resection World J Surg 2010: [Epub ahead of print]

16. Stoot JH, van Dam RM, Busch OR, et al. The effect of a multimodal fast-track programme on outcomes in laparoscopic liver surgery: a multicentre pilot study HPB (Oxford) 2009: 11; 140-144

17. van Dam RM, Hendry PO, Coolsen MM, et al. Initial experience with a multimodal enhanced recovery programme in patients undergoing liver resection Br J Surg 2008: 95; 969-975

18. Dindo D, Demartines N, Clavien PA Classification of surgical complications: a new proposal with evaluation in a cohort of 6336 patients and results of a survey Ann Surg 2004: 240; 205-213

19. Cuesta MA, Meijer S, Paul MA, et al. Limited laparoscopic liver resection of benign tumors guided by laparoscopic ultrasonography: report of two cases Surg Laparosc Endosc 1995: 5; 396-401

20. Berends FJ, Meijer S, Prevoo W, et al. Technical considerations in laparoscopic liver surgery Surg Endosc 2001: 15; 794-798

21. van Keimpema L, Hockerstedt K Treatment of polycystic liver disease Br J Surg 2009: 96; 1379-1380

22. van Keimpema L, Ruurda JP, Ernst MF, et al. Laparoscopic fenestration of liver cysts in polycystic liver disease results in a median volume reduction of 12.5\% J Gastrointest Surg 2008: 12; 477-482

23. Schipper HG, Kager PA Diagnosis and treatment of hepatic echinococcosis: an overview Scand J Gastroenterol Suppl 2004; 50-55

24. de Jong KP, Hesselink EJ, Lameris JS, et al. [Partial liver resection] Ned Tijdschr Geneeskd 1989: 133; 2381-2384

25. de Jong KP, Blankensteijn JD, Hesselink EJ, et al. [Partial hepatectomy for benign or malignant liver diseases; experience in 94 patients] Ned Tijdschr Geneeskd 1989: 133; 2385-2388 


\section{Chapter 11}

26. van Ooijen B, Wiggers $\mathrm{T}$, Meijer S, et al. Hepatic resections for colorectal metastases in The Netherlands. A multiinstitutional 10-year study Cancer 1992: 70; 28-34

27. Cuesta MA, Meijer S, Borgstein PJ, et al. Laparoscopic ultrasonography for hepatobiliary and pancreatic malignancy Br J Surg 1993: 80; 1571-1574

28. van Gulik T Open versus laparoscopic resection for liver tumours HPB (Oxford) 2009: 11; 465-468

29. Miles WF, Paterson-Brown S, Garden OJ Laparoscopic contact hepatic ultrasonography Br J Surg 1992: 79; 419-420

30. Windsor JA, Garden OJ Laparoscopic ultrasonography The Australian and New Zealand journal of surgery 1993: 63; 1-2

31. Nieveen van Dijkum EJ, de Wit LT, van Delden OM, et al. Staging laparoscopy and laparoscopic ultrasonography in more than 400 patients with upper gastrointestinal carcinoma Journal of the American College of Surgeons 1999: 189; 459-465

32. Gouma DJ, de Wit LT, Nieveen van Dijkum E, et al. Laparoscopic ultrasonography for staging of gastrointestinal malignancy Scand J Gastroenterol Suppl 1996: 218; 43-49

33. Cherqui D, Soubrane O, Husson E, et al. Laparoscopic living donor hepatectomy for liver transplantation in children Lancet 2002: 359; 392-396

34. Giulianotti PC, Coratti A, Angelini $M$, et al. Robotics in general surgery: personal experience in a large community hospital Arch Surg 2003: 138; 777-784

35. Vibert E, Denet C, Gayet B Major digestive surgery using a remote-controlled robot: the next revolution Arch Surg 2003: 138; 1002-1006

36. Cherqui D Laparoscopic liver resection Br J Surg 2003: 90; 644-646

37. Shimada M, Hashizume M, Maehara S, et al. Laparoscopic hepatectomy for hepatocellular carcinoma Surg Endosc 2001: 15; 541-544

38. Pulitano $C$, Aldrighetti $L$ The current role of laparoscopic liver resection for the treatment of liver tumors Nat Clin Pract Gastroenterol Hepatol 2008: 5; 648-654

39. Vigano L, Tayar C, Laurent A, et al. Laparoscopic liver resection: a systematic review J Hepatobiliary Pancreat Surg 2009: 16; 410-421

40. Polignano FM, Quyn AJ, de Figueiredo RS, et al. Laparoscopic versus open liver segmentectomy: prospective, case-matched, intention-to-treat analysis of clinical outcomes and cost effectiveness Surg Endosc 2008: 22; 2564-2570

41. Vanounou T, Steel JL, Nguyen KT, et al. Comparing the clinical and economic impact of laparoscopic versus open liver resection Ann Surg Oncol: 17; 998-1009

42. Nguyen KT, Gamblin TC, Geller DA World Review of Laparoscopic Liver Resection-2,804 Patients Ann Surg 2009

43. Hashizume M, Shimada M, Sugimachi K Laparoscopic hepatectomy: new approach for hepatocellular carcinoma J Hepatobiliary Pancreat Surg 2000: 7; 270-275

44. Johnstone PA, Rohde DC, Swartz SE, et al. Port site recurrences after laparoscopic and thoracoscopic procedures in malignancy J Clin Oncol 1996: 14; 1950-1956

45. Gutt CN, Riemer V, Kim ZG, et al. Impact of laparoscopic surgery on experimental hepatic metastases Br J Surg 2001: 88; 371-375

46. Targarona EM, Martinez J, Nadal A, et al. Cancer dissemination during laparoscopic surgery: tubes, gas, and cells World J Surg 1998: 22; 55-60; discussion 60-51

47. Laurent $A$, Cherqui $D$, Lesurtel $M$, et al. Laparoscopic liver resection for subcapsular hepatocellular carcinoma complicating chronic liver disease Arch Surg 2003: 138; 763-769; discussion 769

48. Mala T, Edwin B, Gladhaug I, et al. A comparative study of the short-term outcome following open and laparoscopic liver resection of colorectal metastases Surg Endosc 2002: 16; 1059-1063

49. Cherqui D, Laurent A, Tayar C, et al. Laparoscopic liver resection for peripheral hepatocellular carcinoma in patients with chronic liver disease: midterm results and perspectives Ann Surg 2006: 243; 499-506

50. Teramoto K, Kawamura T, Takamatsu S, et al. Laparoscopic and thoracoscopic partial hepatectomy for hepatocellular carcinoma World J Surg 2003: 27; 1131-1136 
51. Vibert E, Perniceni T, Levard H, et al. Laparoscopic liver resection Br J Surg 2006: 93; 67-72

52. Mala T, Edwin B, Rosseland AR, et al. Laparoscopic liver resection: experience of 53 procedures at a single centre J Hepatobiliary Pancreat Surg 2005: 12; 298-303

53. Dulucq JL, Wintringer $P$, Stabilini $C$, et al. Laparoscopic liver resections: a single centre experience Surg Endosc 2005: 19; 886-891

54. Katkhouda N, Hurwitz M, Gugenheim J, et al. Laparoscopic management of benign solid and cystic lesions of the liver Ann Surg 1999: 229; 460-466 



\section{Chapter 12}

\section{A survey in the HPB community on ways to enhance patient recovery}

E.M. Wong-Lun-Hing, T.M. Lodewick, J.H.M.B. Stoot, M.H.A. Bemelmans, S.W.M. Olde Damink, C.H.C Dejong, R.M. van Dam

Adapted from HPB (in press)

Presented to the European-African Hepato-Pancreato-Biliary Association in Cape Town, South Africa, April 2011. 


\section{ABSTRACT}

Background: Both laparoscopic techniques and multimodal enhanced recovery programmes have been shown to improve recovery and reduce length of hospital stay. Interestingly, evidence-based care programmes are not widely implemented, whereas new, minimally invasive surgical procedures are often adopted with very little evidence to support their effectiveness. The present survey aimed to shed light on experiences of the adoption of both methods of optimizing recovery.

Methods: An international web-based, 18 question, electronic survey was composed in 2010. The survey was sent out to 673 hepatopancreatobiliary (HPB) centres worldwide in June 2010 to investigate worldwide experience with laparoscopic liver surgery, fast-track recovery programmes, and surgeryrelated equipoise for the open and laparoscopic techniques and to assess opinions on strategies for adopting laparoscopic liver surgery in HPB surgical practice.

Results: A total of 507 centres responded (response rate: 75.3\%), 161 of which finished the survey completely. All units reported performing open liver resections, 24.2\% performed open living donor resections, 39.1\% carried out orthotopic liver transplantations, $87.6 \%$ had experience with laparoscopic resections and $2.5 \%$ performed laparoscopic living donor resections. A median of 50 (range: 2-560) open and 9.5 (range: 1-80) laparoscopic liver resections per surgical unit were performed in 2009. Patients stayed in hospital for a median of 7 days (range: 2-15 days) after uncomplicated open liver resection and a median of 4 days (range: 1-10 days) after uncomplicated laparoscopic liver resection. Only $28.0 \%$ of centres reported having experience with fast-track programmes in liver surgery. The majority considered the instigation of a randomized controlled trial or a prospective register comparing the outcomes of open and laparoscopic techniques to be necessary.

Conclusion: Worldwide dissemination of laparoscopic liver resection is substantial, although laparoscopic volumes are low in the majority of HPB centres. The adoption of enhanced recovery programmes in liver surgery is limited and should be given greater attention. 


\section{INTRODUCTION}

In recent years laparoscopic liver resection and enhanced recovery programmes have been introduced in liver surgery with the aim of accelerating postoperative recovery and shortening hospital length of stay (LoS). Like open liver resection, laparoscopic resection of liver lesions can be applied safely in both malignant and benign lesions. ${ }^{1-7}$ Large prospective case series suggest that laparoscopic liver surgery might be superior to open liver surgery in terms of perioperative blood loss, postoperative pain, time to recovery, LoS, cosmetic appearance and quality of life. ${ }^{5,8-10}$ Survival rates after laparoscopic and open resection of hepatocellular carcinoma and hepatic colorectal metastases seem comparable. ${ }^{1,5}$

Similarly, fast-track programmes have proven to be useful, feasible and safe in liver surgery. ${ }^{11-15}$ Such programmes can also enhance recovery and reduce LoS. The Enhanced Recovery After Surgery (ERAS) programmes rely mainly on optimising perioperative care and reducing the stress responses to surgery though the provision of adequate preoperative patient counselling, optimised anaesthesia and analgesia, quick resumption of oral intake and early mobilisation. ${ }^{12,16-18}$ In liver resection, earlier resumption of oral intake, faster postoperative recovery and a significant reduction in median LoS (from 8 to 6 days) was shown when patients were managed within a multimodal ERAS programme. ${ }^{12}$

A small pilot study in liver surgery suggested that laparoscopic liver surgery within an ERAS setting led to a potentially accelerated recovery and further reduction in LoS from 7 to 5 days. ${ }^{11}$ Moreover, combining laparoscopy and an ERAS strategy is most likely to result in a synergetic effect, as recently proven in a randomised controlled trial (RCT) in the context of colonic surgery. ${ }^{19}$

Despite the fairly robust evidence that many specific elements of fast-track programmes can enhance recovery and reduce LoS, little evidence on the use of these programmes in liver surgery has been published. This suggests that adoption of ERAS programmes in liver surgery worldwide is low. Current surgical practice is based on evidence and any change in daily routines should be supported by sound data. ${ }^{20}$ In this respect, the current fairly liberal adoption of laparoscopic liver surgery contrasts with the relative lack of adoption of enhanced recovery programmes.

An international web-based survey was composed to evaluate worldwide experience with laparoscopic liver surgery and fast-track recovery programmes, and surgery-related equipoise in open and laparoscopic strategies, and to assess opinions on strategies for the adoption of laparoscopic liver surgery in daily hepatopancreatobiliary (HPB) surgical practice. 


\section{METHODS}

Study design. An online electronic survey, consisting of 18 questions subdivided according to five main domains, was developed (Appendix 1). Questions on the different topics were initially composed by two research fellows (E.M.W.L.H. and T.M.L.) and two liver surgeons (R.M.v.D. and J.H.M.B.S.). The definitive set of questions was then administered using SurveyMonkey ${ }^{\mathrm{TM}}$ (Palo Alto, California, USA). Items in the first domain included several questions on experience in open and laparoscopic liver surgery and covered types and numbers of liver resections. Items in the second domain concerned recovery and LoS after uncomplicated open or laparoscopic liver resection. Items in the third domain surveyed experience with enhanced recovery or fast-track perioperative care programmes such as ERAS programmes. The fourth part of the survey was developed to evaluate opinions on the necessity of an RCT and on the value of a prospective registry comparing outcomes in open and laparoscopic liver surgery. Items in the final domain evaluated current opinions on and considerations for participating in such a trial. Incomplete responses were excluded from analysis.

Study population. An invitation to complete this survey was sent by e-mail to 673 HPB centres worldwide in June 2010. Subsequent e-mail reminders were sent out in August and September 2010. Only one surgeon per HPB unit was asked to participate. Figure 1 describes the respondent inclusion and exclusion process. The participation period closed and analyses were conducted in November 2010. 


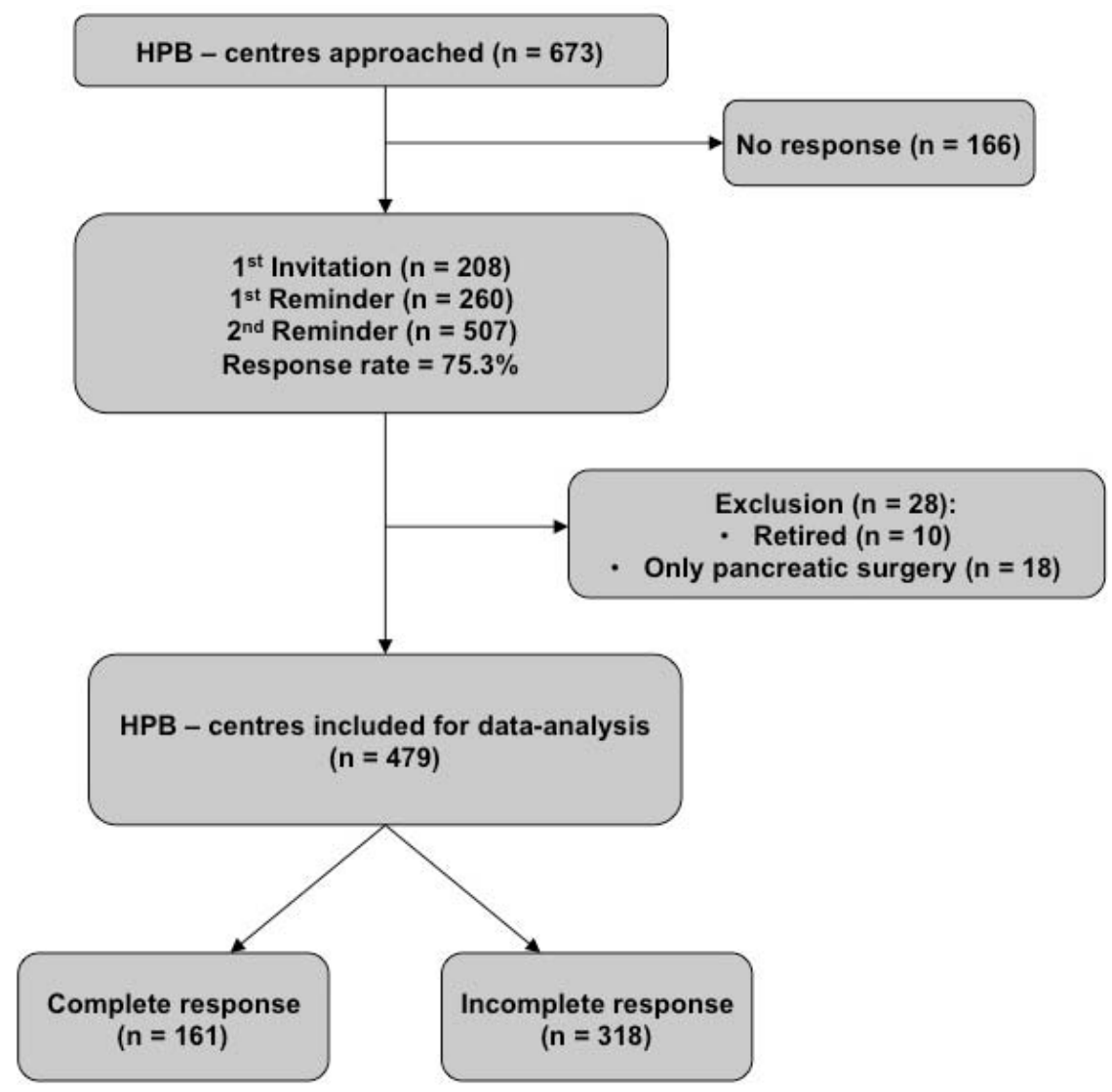

Figure 1. Flowchart showing the respondent inclusion and exclusion process. A total of 673 hepatopancreatobiliary (HPB) centres were approached. Cumulative response numbers after the first invitation and first and second reminders are displayed. A total of 166 surgeons did not respond to the study invitation or reminders, and data from an additional 28 centres were excluded. Only data from the centres that provided complete responses $(n=161)$ were analysed

Statistics. Survey data were extracted into an excel database. Statistical analysis was performed using the Statistical Package for the Social Sciences (SPSS) version 18.0 and GraphPad Prism version 5 (GraphPad Software, Inc., La Jolla, CA, USA). Basic analyses were performed using descriptive statistics including counts, percentages, means with standard deviations and medians with ranges and interquartile ranges. Subgroup analysis was performed to investigate potentially relevant differences between regions and centre experience (Mann-Whitney $U$ test or $\chi 2$ ). All countries were assigned to one of the following six regions: Europe; North America; Middle and South America; Asia; Oceania and Africa. An experienced laparoscopic HPB centre was defined as a surgical unit performing 20 or more laparoscopic liver resections annually. ${ }^{21}$ 


\section{RESULTS}

Participation. A total of 507 centres (one surgeon per centre) responded (response rate $75.3 \%$ ). Incomplete responders were excluded for data analysis, leaving 161 centres that completed the survey. Worldwide centres from 39 different countries participated; these were divided into groups according to the six global regions (fig.2). The regions that provided the highest response rates Europe (45\%) and North-America (26\%), with the USA $(n=34)$, Italy $(n=16)$, Canada $(n=8)$ and the Netherlands $(n=8)$ representing the top 4 countries providing complete responses.

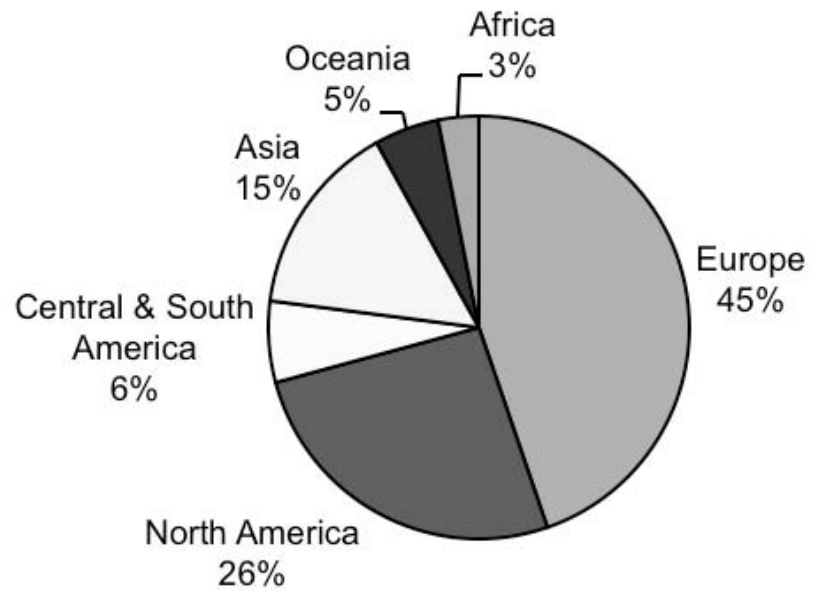

Figure 2. Centres $(n=161)$ included in the data- analysis by region.

Types of liver resections. Open resection for liver lesions was performed by $100 \%$ of the units. $87.6 \%$ of the responding units reported experience with laparoscopic resections of liver lesions, $39.1 \%$ with orthotopic liver transplantations, $24.2 \%$ with open living donor resections and $2.5 \%$ with laparoscopic living donor resections. Table 1 shows the percentage of HPB-centres performing different types of liver surgery and the differences between regions. 
Table 1. Centres performing types of procedure, by region

\begin{tabular}{|c|c|c|c|c|c|c|c|}
\hline & Centres & performir & ng surgical & procedur & es, by regi & on $n(\%)$ & \\
\hline & Europe & $\begin{array}{l}\text { North } \\
\text { America }\end{array}$ & $\begin{array}{l}\text { Central \& } \\
\text { South } \\
\text { America }\end{array}$ & Asia & Oceania & Africa & $\begin{array}{l}\text { World- } \\
\text { wide }\end{array}$ \\
\hline Open resection of liver lesions & $\begin{array}{l}72 / 72 \\
(100.0)\end{array}$ & $\begin{array}{l}42 / 42 \\
(100.0)\end{array}$ & $\begin{array}{l}10 / 10 \\
(100.0)\end{array}$ & $\begin{array}{l}24 / 24 \\
(100.0)\end{array}$ & $\begin{array}{l}8 / 8 \\
(100.0)\end{array}$ & $\begin{array}{l}5 / 5 \\
(100.0)\end{array}$ & $\begin{array}{l}161 / 161 \\
(100.0)\end{array}$ \\
\hline Lap. resection of liver lesions & $\begin{array}{l}61 / 72 \\
(84.7)\end{array}$ & $\begin{array}{l}41 / 42 \\
(97.6)\end{array}$ & $\begin{array}{l}10 / 10 \\
(100.0)\end{array}$ & $\begin{array}{l}19 / 24 \\
(79.2)\end{array}$ & $\begin{array}{l}7 / 8 \\
(87.5)\end{array}$ & $\begin{array}{l}3 / 5 \\
(60.0)\end{array}$ & $\begin{array}{l}141 / 161 \\
(87.6)\end{array}$ \\
\hline Orthotopic liver transplantation & $\begin{array}{l}29 / 72 \\
(40.2)\end{array}$ & $\begin{array}{l}17 / 42 \\
(40.5)\end{array}$ & $\begin{array}{l}5 / 10 \\
(50.0)\end{array}$ & $\begin{array}{l}7 / 24 \\
(29.2)\end{array}$ & $\begin{array}{l}4 / 8 \\
(50.0)\end{array}$ & $\begin{array}{l}1 / 6 \\
(20.0)\end{array}$ & $\begin{array}{l}63 / 161 \\
(39.1)\end{array}$ \\
\hline Open living donor resection & $\begin{array}{l}15 / 72 \\
(20.8)\end{array}$ & $\begin{array}{l}10 / 42 \\
(23.8)\end{array}$ & $\begin{array}{l}4 / 10 \\
(40.0)\end{array}$ & $\begin{array}{l}7 / 24 \\
(29.2)\end{array}$ & $\begin{array}{l}2 / 8 \\
(25.0)\end{array}$ & $\begin{array}{l}1 / 5 \\
(20.0)\end{array}$ & $\begin{array}{l}39 / 161 \\
(24.2)\end{array}$ \\
\hline Lap. Living donor resection & $\begin{array}{l}1 / 72 \\
(1.4)\end{array}$ & $\begin{array}{l}1 / 42 \\
(2.4)\end{array}$ & $\begin{array}{l}1 / 10 \\
(10.0)\end{array}$ & $\begin{array}{l}1 / 24 \\
(4.2)\end{array}$ & $\begin{array}{l}0 / 8 \\
(0.0)\end{array}$ & $\begin{array}{l}0 / 5 \\
(0.0)\end{array}$ & $\begin{array}{l}4 / 161 \\
(2.5)\end{array}$ \\
\hline
\end{tabular}

Experience. A total of $42.0 \%$ of responding centres indicated that their data represented precise numbers. The remaining centres provided estimations that were as accurate as possible. A wide range in the number of resections performed was observed among HPB centres. In 2009, the median number of open resections for liver lesions performed was 50 (range: $2-560$; IQR $=50$ ) per surgical unit. In the same year, the median number of laparoscopic resections of liver lesions performed was 9.5 (range: $1-80$; IQR = 15) per centre. Worldwide figures for open and laparoscopic liver resections and differences among regions are shown in Table 2. Of the participating centres, $26.6 \%$ could be classified as experienced laparoscopic liver centres based on their completion of at least 20 laparoscopic liver resections per year (Table 3). Experienced laparoscopic centres seemed to be more frequently located in the Americas than in other continents ( $44.2 \%$ vs. $13.5 \% ; P<0.001)$.

Table 2. Number of resections per surgical unit per year (2009)

\begin{tabular}{lll}
\hline \multirow{2}{*}{ Region } & \multicolumn{2}{l}{ Liver resections performed in 2009, median (range)/IQR } \\
\cline { 2 - 3 } & Open liver resections & \multicolumn{2}{l}{ Laparoscopic liver resections } \\
\hline Europe & $55.0(2-250) / 60.0$ & $5.0(1-61) / 9.0$ \\
North America & $45.0(6-200) / 42.5$ & $19.0(2-80) / 21.0$ \\
Central \& South America & $32.5(12-80) / 23.5$ & $10.0(3-30) / 18.0$ \\
Asia & $50.0(5-560) / 62.3$ & $6.0(1-80) / 5.0$ \\
Oceania & $57.5(15-150) / 110.0$ & $9.0(1-20) / 7.0$ \\
Africa & $50.0(5-120) / 115.0$ & $4.5(3-13) / 7.8$ \\
Worldwide & $50.0(2-560) / 50.0$ & $9.5(1-80) / 15.0$ \\
\hline
\end{tabular}

IQR, interquartile range. 
Table3. Numbers of laparoscopic liver surgery centres demonstrating experience defined by a volume of >20 laparoscopic resections per year

\begin{tabular}{ll}
\hline Region & $\begin{array}{l}\text { Experienced laparoscopic liver centres, } n / \text { laparoscopic liver centres, } \\
n(\%)\end{array}$ \\
\hline Europe & $10 / 59(16.9 \%)$ \\
North America & $20 / 41(48.8 \%)$ \\
Central \& south America & $3 / 9(33.3 \%)$ \\
Asia & $3 / 19(15.8 \%)$ \\
Oceania & $1 / 7(14.3 \%)$ \\
Africa & $0 / 4$ \\
Worldwide & $37 / 139(26.6 \%)$ \\
\hline
\end{tabular}

Hospital length of stay and fast-track perioperative care programmes. The reported median hospital LoS after uncomplicated liver resection was 7 days (range: 2-15 days) after open surgery and 4 days (range: 1-10 days) after laparoscopic surgery. Differences among regions are shown in Table 4. In addition, a subgroup analysis showed that experienced centres achieved a significantly shorter median LoS after laparoscopic liver resection compared with inexperienced centres [3.4 days (range: $1-7$ days) vs. 4.2 days (range: $1-10$ days); $P=0.013$ ]. Half of the HPB centres $(50.1 \%)$ had experience with fast-track perioperative care programmes in colonic, hepatic or pancreatic surgery or a combination of these fields. Only $28.0 \%$ had experience with these programmes in liver surgery. Results per region are shown in Table 5.

\section{Necessity for an RCT comparing open and laparoscopic liver surgery}

The majority (59.4\%) of HPB centres considered that an RCT comparing outcomes in open and laparoscopic liver surgery prior to the further adoption of laparoscopic liver surgery was necessary. A total of $49.1 \%$ considered that a combination of such an RCT and a prospective multicentre registry should be mandatory; $36.4 \%$ considered that a prospective multicentre registry alone would be sufficient and $4.2 \%$ deemed a prospective hospital registry adequate. Of the surgical units that performed both open and laparoscopic left lateral sectionectomy (LLS) within a fasttrack/ERAS programme, $82.9 \%$ indicated that they would consider participating in an RCT. Level A evidence to support the superiority of the laparoscopic technique was still considered necessary by the majority of respondents. A total of $94.3 \%$ of participants with experience in both open and laparoscopic LLS, both within and outwith fast-track or ERAS programmes, would also consider participating in a prospective registry. Overall, $\mathbf{8 3 . 3 \%}$ of liver units without experience in laparoscopic liver surgery indicated a desire to participate in hands-on training in laparoscopic liver surgery and/or a proctor programme. 
Table 4. Length of stay after uncomplicated liver resection

\begin{tabular}{|c|c|c|}
\hline \multirow[t]{2}{*}{ Region } & \multicolumn{2}{|c|}{ Length of stay, days, median (range)/IQR } \\
\hline & After open liver resection & After laparoscopic liver resection \\
\hline Europe & $7.0(4-12) / 3.0$ & $5.0(2-10) / 1.0$ \\
\hline North America & $5.0(4-8) / 2.0$ & $3.0(1-5) / 1.5$ \\
\hline Central \& South America & $5.0(2-7) / 1.8$ & $2.5(1-5) / 2.0$ \\
\hline Asia & $7.0(5-15) / 2.0$ & $4.0(3-10) / 2.0$ \\
\hline Oceania & $5.0(3-7) / 1.8$ & $4.0(2-5) / 1.5$ \\
\hline Africa & $8.0(4-10) / 5.5$ & $4.5(2-7) / 4.5$ \\
\hline Worldwide & $7.0(2-15) / 3.0$ & $4.0(1-10) / 2.0$ \\
\hline
\end{tabular}

Table5. Centres with experience in fast-track perioperative care programmes

\begin{tabular}{lllll}
\hline Region & \multicolumn{4}{l}{ Experience with ERAS programmes in a specific type of surgery, $n /$ total $n(\%)$} \\
\cline { 2 - 5 } & No experience & In colon surgery & In liver surgery & In pancreatic surgery \\
\hline Europe & $31 / 72(43.1 \%)$ & $36 / 72(50.0 \%)$ & $22 / 72(30.6 \%)$ & $14 / 72(19.4 \%)$ \\
North America & $25 / 42(59.5 \%)$ & $6 / 42(14.3 \%)$ & $9 / 42(21.4 \%)$ & $8 / 42(19.0 \%)$ \\
Central \& South America & $5 / 10(50.0 \%)$ & $4 / 10(40.0 \%)$ & $3 / 10(30.0 \%)$ & $1 / 10(10.0 \%)$ \\
Asia & $15 / 24(62.5 \%)$ & $8 / 24(33.3 \%)$ & $4 / 24(16.7 \%)$ & $3 / 24(12.5 \%)$ \\
Oceania & $2 / 8(25.0 \%)$ & $5 / 8(62.5 \%)$ & $6 / 8(75.0 \%)$ & $2 / 8(25.0 \%)$ \\
Africa & $4 / 5(80.0 \%)$ & $1 / 5(20.0 \%)$ & $1 / 5(20.0 \%)$ & $0 / 5$ \\
Worldwide & $82 / 161(50.9 \%)$ & $60 / 161(37.3 \%)$ & $45 / 161(28.0 \%)$ & $28 / 161(17.4 \%)$ \\
\hline
\end{tabular}

\section{DISCUSSION}

This study aimed to assess the worldwide experience and dissemination of two recently introduced strategies to accelerate recovery after liver surgery. It demonstrates that the majority of HPB centres perform liver surgery in the absence of an enhanced recovery perioperative care programme, and that the majority of HPB centres perform laparoscopic liver surgery. This study also shows a substantial variance in hospital length of stay among centres and regions. Lastly, this study demonstrates clinical equipoise for laparoscopic and open liver resection is present in the HPB community. ${ }^{22,23}$ Clinical equipoise refers to a context in which there is no preference or certainty of therapeutic equivalence for either laparoscopic or open liver surgery. The majority of liver centres considered that an RCT trial was necessary to prove the laparoscopic technique to be equal or superior to open surgery.

The results show a high level of dissemination of laparoscopic liver surgery. Both low- and high-volume centres, amounting to $87.6 \%$ of HPB units, perform liver resections laparoscopically. Although the laparoscopic procedure is frequently used, many centres in this study have limited experience in laparoscopic liver resection. By contrast, responding centres displayed limited adoption of enhanced recovery programmes in liver surgery (one in four). The liberal adoption of laparo- 
scopic liver surgery, even in low-volume HPB centres, is seemingly in conflict with current standards of evidence-based practice in the medical community. Neither is it in keeping with recommendations expressed in an expert consensus (the Louisville Consensus) which concluded that laparoscopic liver surgery was safe and effective in the hands of experienced and trained surgeons. ${ }^{24}$ In line with the available evidence for fast-track principles in liver surgery, ${ }^{11-15}$ proof of the merits of laparoscopic vs. open liver surgery is also limited and no RCTs have been undertaken. However, a recent literature review and meta-analysis of available prospective and retrospective studies comparing open with laparoscopic liver resections both found both short- and long-term outcome seems favourable for the laparoscopic procedure. Not only was LoS markedly shorter, but blood loss and complications were found to be reduced and oncologic outcomes did not differ between the two techniques. ${ }^{25,26}$

Low-volume centres in this study reported a significantly longer hospital LoS compared to high-volume centres. In addition, LoS after open and laparoscopic liver surgery varied substantially among regions. The surgeons progress along the laparoscopic learning curve influence LoS, because laparoscopic liver resection is technically demanding and requires expertise in both advanced laparoscopic skills and open liver surgery. ${ }^{27}$ This is in line with the findings of a meta-analysis of studies reporting on 20 or more laparoscopic procedures, which indicated that a laparoscopic approach led to a significant reduction in morbidity and LoS. ${ }^{21}$ In addition, differences in standard of care and discharge criteria may also contribute to variance in LoS reported in literature (3-20 days after open and 6-32 days after laparoscopic liver resection). ${ }^{26}$ Substantial distinctions at a cultural level may lie at the root of the observed variance in LoS. In some regions patients are discharged to a home care institution early in their recovery process (e.g. in North America), whereas in other world regions the provision of protocol-based care and the associated logistics may be lacking. This may lead to a difference in expectations on both the patient's and surgeon's part as to when a patient might be ready for discharge. Thus, LoS is a poor outcome parameter that hampers comparison and is hard to interpret. The implementation of a structured care programme with welldefined recovery and discharge criteria, as used within ERAS protocols, might improve the comparability of clinical outcomes in future (multicentre) trials.

Laparoscopy and enhanced recovery programmes should not be seen as separate methods of improving postoperative recovery and outcomes such as morbidity rates and LoS. On the contrary, it is likely that the implementation of both will result in a synergetic improvement. Enhanced recovery programmes in liver surgery have already been shown to reduce hospital LoS. ${ }^{12-15}$ The additional benefit of an ERAS strategy in a laparoscopic setting was recently demonstrated in a trial in colonic surgery and a small pilot study in liver surgery. ${ }^{11,19}$

This worldwide survey unveils experiences in enhanced recovery programmes 
and laparoscopic liver surgery, and deliberations on the need for RCTs in liver surgery. Although the present study achieved a response rate of $>75 \%$, only $23.9 \%$ of respondents completed the survey in full, which limited the study findings. However, representatives of 161 liver surgery centres worldwide shared their results and opinions. The use of a survey may be seen as limiting the study because results are strongly dependent on the type of respondents, questions and response rate. In addition, the group profile of responding centres may have been subject to response bias. Centres of considerable volume and those performing laparoscopic liver surgery may have been more likely to respond.

The issue of import does not concern a choice between laparoscopic liver resection and an ERAS strategy, but, rather, how both paths can be adequately adopted. The majority of responders still consider an RCT of laparoscopic surgery to be necessary. It could be argued that as more liver centres adopt laparoscopic techniques, opportunities to conduct an RCT may be diminished by patient and surgeon preferences. According to some surgeons, laparoscopic liver resection is without doubt therapeutically superior, whereas for others this remains to be proven. In addition, in the Louisville Consensus of 2008, experts concluded that the accrual of patients for an RCT would be slow as a result of low overall numbers. The ORANGE II Trial, currently enrolling patients, will be the first RCT (combined with a prospective registry) to provide evidence on laparoscopic vs. open liver resection. ${ }^{28}$ As for enhanced recovery programmes, many centres are likely to have already implemented multiple ERAS elements as part of modern care. Further trials are

needed to assess compliance with recovery protocols, ${ }^{29,30}$ specific elements of enhanced recovery programmes and the possible reduction of medical expenses.

\section{Conclusions}

The worldwide dissemination of laparoscopic liver resection is substantial, although the average volume of laparoscopic resections carried out in the majority of HPB centres is low. The adoption of enhanced recovery programmes in liver surgery is limited and the issue warrants greater attention. Both strategies are associated with faster recovery and may work synergistically. Given the increasing strength of the role of evidence-based medicine in current surgical practice, more evidence is required.

Acknowledgements. We thank Simon A. Dello and Robert J.S. Coelen for helping design the survey. 


\section{REFERENCES}

1. Nguyen KT, Gamblin TC, Geller DA. World review of laparoscopic liver resection - 2804 patients. Ann Surg. 2009; 250:831-841.

2. Abu Hilal M, Pearce NW. Laparoscopic left lateral liver sectionectomy: a safe, efficient, reproducible technique. Dig Surg 2008;25:305-308.

3. Dagher I, Lainas P, Carloni A, Caillard C, Champault A, Smadja C et al. Laparoscopic liver resection for hepatocellular carcinoma. Surg Endosc 2008;22:372-378.

4. Dagher I, Proske JM, Carloni A, Richa H, Tranchart H, Franco D. Laparoscopic liver resection: results for 70 patients. Surg Endosc 2007; 21:619-624.

5. Lee KF, Cheung YS, Chong CN, Tsang YY, Ng WW, Ling E et al. Laparoscopic versus open hepatectomy for liver tumours: a case-control study. Hong Kong Med J 2007;13:442-448.

6. O’Rourke N, Fielding G. Laparoscopic right hepatectomy: surgical technique. J Gastrointest Surg 2004;8:213-216.

7. Troisi R, Montalti R, Smeets $\mathrm{P}$, van Huysse J, van Vlierberghe $H$, Colle I et al. The value of laparoscopic liver surgery for solid benign hepatic tumours. Surg Endosc 2008;22:38-44.

8. Laurence JM, Lam VW, Langcake ME, Hollands MJ, Crawford MD, Pleass HC. Laparoscopic hepatectomy, a systematic review. ANZ J Surg 2007;77:948-953.

9. Abu Hilal M, McPhail MJ, Zeidan B, Zeidan S, Hallam MJ, Armstrong $T$ et al. Laparoscopic versus open left lateral hepatic sectionectomy: a comparative study. Eur J Surg Oncol 2008;34:1285-1288.

10. Alkari B, Owera A, Ammori BJ. Laparoscopic liver resection: preliminary results from a UK centre. Surg Endosc 2008;22:2201-2207.

11. Stoot JH, van Dam RM, Busch OR, van Hillegersberg R, De Boer M, Olde Damink SW et al. The effect of a multimodal fast-track programme on outcomes in laparoscopic liver surgery: a multicentre pilot study. HPB 2009;11:140-144.

12. van Dam RM, Hendry PO, Coolsen MM, Bemelmans MH, Lassen K, Revhaug A et al. Initial experience with a multimodal enhanced recovery programme in patients undergoing liver resection. $\mathrm{Br} J$ Surg 2008; 95:969-975.

13. Lin DX, Li X, Ye QW, Lin F, Li LL, Zhang QY. Implementation of a fast-track clinical pathway decreases postoperative length of stay and hospital charges for liver resection. Cell Biochem Biophys 2011;61:413-419.

14. MacKay G, O’Dwyer PJ. Early discharge following liver resection for colorectal metastases. Scott Med J 2008;53:22-24.

15. Spelt L, Ansari D, Sturesson C, Tingstedt B, Andersson R. Fasttrack programmes for hepatopancreatic resections: where do we stand? HPB 2011;13:833-838.

16. Kehlet $\mathrm{H}$, Wilmore DW. Multimodal strategies to improve surgical outcome. Am J Surg 2002;183:630-641.

17. Lassen K, Soop M, Nygren J, Cox PB, Hendry PO, Spies C et al. Consensus review of optimal perioperative care in colorectal surgery: Enhanced Recovery After Surgery (ERAS) Group recommendations. Arch Surg 2009;144:961-969.

18. Wind J, Polle SW, Fung Kon Jin PH, Dejong CH, von Meyenfeldt MF, Ubbink DT et al. Systematic review of enhanced recovery programmes in colonic surgery. Br J Surg 2006;93:800-809.

19. Vlug MS, Wind J, Hollmann MW, Ubbink DT, Cense HA, Engel AF et al. Laparoscopy in combination with fast track multimodal management is the best perioperative strategy in patients undergoing colonic surgery: a randomized clinical trial (LAFA study). Ann Surg 2011;254:868-875.

20. Ubbink DT, Legemate DA. Evidence-based surgery. Br J Surg 2004;91:1091-1092.

21. Simillis C, Constantinides VA, Tekkis PP, Darzi A, Lovegrove R, Jiao L et al. Laparoscopic versus open hepatic resections for benign and malignant neoplasms - a meta-analysis. Surgery 2007;141:203211.

22. Freedman B. Equipoise and the ethics of clinical research. N Engl J Med 1987;317:141-145. 
23. Rodrigues HC, Oerlemans AJ, van den Berg PP. [The need for uncertainty in clinical research equipoise.] Ned Tijdschr Geneeskd 2011;155:A3846.

24. Buell JF, Cherqui D, Geller DA, O'Rourke N, lannitti D, Dagher I et al. The international position on laparoscopic liver surgery: the Louisville Statement, 2008. Ann Surg 2009;250:825-830.

25. Mirnezami R, Mirnezami AH, Chandrakumaran K, Abu Hilal M, Pearce NW, Primrose JN et al. Shortand longterm outcomes after laparoscopic and open hepatic resection: systematic review and metaanalysis. HPB 2011;13:295-308.

26. Nguyen KT, Marsh JW, Tsung A, Steel JJ, Gamblin TC, Geller DA. Comparative benefits of laparoscopic vs. open hepatic resection: a critical appraisal. Arch Surg 2011;146:348-356.

27. van Gulik T. Open versus laparoscopic resection for liver tumours. HPB 2009;11:465-468.

28. van Dam RM, Wong-Lun-Hing EM, van Breukelen GJ, Stoot JH, van der Vorst JR, Bemelmans MH et al. Open versus laparoscopic left lateral hepatic sectionectomy within an enhanced recovery ERAS programme (ORANGE II Trial): study protocol for a randomized controlled trial. Trials 2012;13:54.

29. Maessen J, Dejong CH, Hausel J, Nygren J, Lassen K, Andersen J et al. A protocol is not enough to implement an enhanced recovery programme for colorectal resection. Br J Surg 2007;94:224-231.

30. Spanjersberg WR, Reurings J, Keus F, van Laarhoven CJ. Fast track surgery versus conventional recovery strategies for colorectal surgery. Cochrane Database Syst Rev 2011;(2):CD007635. 


\section{Chapter 12}

\section{APPENDIX 1. ONLINE SURVEY QUESTIONS}

\section{Online ORANGE-II Trial Survey}

\section{Introduction}

*1. I perform liver surgery as part of my daily practice?

$\square$ Yes

$\square$ No (this choice will terminate the survey)

Comment

\section{*2. Personal information:}

Name:

Preferred e-mail address:

\section{Liver resection experience}

*3. Does your surgical unit perform liver resections?

$\square$ Yes, my colleague(s)

$\square$ Yes, I perform them myself

$\square$ No

\section{Liver resection experience II}

*4. What tye of liver resections does your surgical unit perform? (more than one option possible) $\square$ Open resection for liver lesions

$\square$ Open living donor resections $\square$ Orthotopic liver transplantations $\square$ Laparoscopic resection for liver lesions

$\square$ Laparoscopic living donor resections

*5. Does your surgial unit perform open and / or laparoscopic Left Lateral Sectionectomies (LLS)?

$\square$ Yes, open and laparoscopic LLS

$\square$ Yes, but only open LLS

$\square$ Yes, but only laparoscopic LLS

$\square$ No, we do not perform LLS

*6. How many liver resections were performed in your surgical unit during 2009?

Open resection for liver lesions

Open living donor resections

Orthotopic liver transplantations Laparoscopic resection for liver lesions Laparoscopic living donor resections

*7. Are the numbers in the previous question estimates or true numbers from a registry?

$\square$ Estimates

$\square$ True numbers

Comment 


\section{Recovery and Hospital length of stay}

*8. What is the usual hospital length of stay after an UNCOMPLICATED liver resection in your unit?

Open resections in days

Laparoscopic resections in days

*9. When patients are recovered they are discharged to (more than one option possible)

$\square$ Home

$\square$ Supported care institution

$\square$ Care hotel

\section{Enhanced Recovery}

*10. Does your surgical unit have experience with a so called enhanced recovery or fast-track perioperative care programme (ERAS)? (more than one option possible)

$\square$ No

$\square$ Yes, in colon surgery

$\square$ Yes, in liver surgery

$\square$ Yes, in pancreatic surgery

Comment

\section{Enhanced Recovery Experience II}

*11. If your surgical unit has NO experience with an enhanced recovery ERAS programme in liver surgery would you desire implementation of such a programme?

$\square$ Yes

$\square$ No

Comment

\section{Randomised trial and / or prospective registry}

*12. Is in your opinion a randomised controlled trial comparing open and laparoscopic liver surgery necessary before laparoscopic liver surgery can be adopted worldwide?

$\square$ Yes, a randomised trial is necessary

$\square$ Yes, a randomised trial and a prospective multicentre registry are necessary

$\square$ No, a prospective hospital registry is sufficient

Comment

\section{Randomised trial and prospective registry}

*13. If your surgical unit performs both open as well as laparoscopic LLS WITHIN a Fast Track / ERAS programme, would you consider participation in the ORANGE II - Trial?

$\square$ Yes

$\square$ No, because:

*14. If your surgical unit performs both open as well as laparoscopic LLS WITHIN or WITHOUT a Fast Track / ERAS programme, would you consider participation in a prospective registry?

$\square$ Yes 


\section{Chapter 12}

$\square$ No

Comment

*15. If you are willing to participate in the ORANGE II - Trial, would you consider randomisation between open and laparoscopic resection?

$\square$ Yes

$\square$ No, because:

*16. If your unit has no experience with laparoscopic liver surgery would you participate in the registration arm of the ORANGE II - Trial?

$\square$ Yes

$\square$ No

Comment

*17. If the ORANGE II - Trial would offer you the option of online eCRF completion, would this lower the bar for participation?

$\square$ Yes

$\square$ No

Comment

\section{Training and Credentialing}

*18. If your unit has no experience with laparoscopic liver surgery would you want to be trained in a hands-on and / or proctor programme?

$\square$ Yes

$\square$ No

Comment 


\section{Part 5}

\section{Future perspectives}


Part 5 


\section{INTRODUCTION}

Future studies on improving the outcome of liver surgery are a central theme in this part. This will be addressed in three chapters. First, a tool for surgeons to help preventing post resectional liver failure by means of liver volumetric assessment prior to resection is evaluated in chapter 13.

Second, the role of reducing the size of liver adenomas to avoid unnecessary surgery as explored in the previous study (chapter 4) will be further investigated. Having shown shrinkage of liver adenomas not only after stopping OAC but also after selective arterial embolisation of the haemorrhaged adenomas, the question arose whether this could be a therapy in selected cases of large hepatic adenomas. In order to provide the best level of evidence a randomised trial is proposed to compare between two invasive techniques: embolisation with radiological followup and liver resection.

Third, as discussed earlier, a multicentre pilot study in the Netherlands showed that laparoscopic liver surgery conducted in a multimodal enhanced recovery programme resulted in earlier functional recovery than the same surgical approach in traditional care setting. Since more is to be expected from the synergy between the minimally invasive approach and ERAS, an outline is proposed for a new international multicentre trial: the laparoscopic approach for liver surgery will be compared to open surgery, both within a multimodal perioperative ERAS programme. 



\section{Chapter 13}

\section{Prospective volumetric assessment of the}

liver on a personal computer by non radiologists prior to partial hepatectomy

S.A.W.G. Dello, J.H.M.B. Stoot, R.S.A. van Stiphout, J.G. Bloemen, S.J. Wigmore, C.H.C. Dejong, R.M. van Dam

Adapted from World J Surg. 2011 Feb;35(2):386-92

Presented to the 9th World Congress of the International Hepato-Pancreato-Biliary Association in Buenos Aires, Argentina, April 2010. 


\section{ABSTRACT}

Background: A small remnant liver volume is an important risk factor for posthepatectomy liver failure. ImageJ and $\mathrm{OsiriX}^{\circledR}$ are both free, open-source image processing software packages. The aim of the present study was to compare ImageJ and OsiriX ${ }^{\circledR}$ in performing prospective CT-volumetric analysis of the liver on a Personal Computer in patients undergoing major liver resection.

Methods: Patients scheduled for a right hemihepatectomy were eligible for inclusion. Two surgeons and one surgical trainee measured volumes of total liver, tumour and future resection specimen prospectively with ImageJ and OsiriX ${ }^{\circledR}$. A radiologist also measured these volumes with CT scanner-linked Aquarius iNtuition ${ }^{\circledR}$ software. Resection volumes were compared with the actual weights of the liver specimens removed during surgery and differences between the measured liver volumes were analyzed.

Results: A total of 15 patients ( 8 men, 7 women) with a median age of 63 years (4879 years) were included. There was a significant correlation between measured weights of resection specimens and the volumes calculated prospectively with ImageJ and Osiri $X^{\circledR}(r=0.89 ; r=0.83$, respectively). There was also a significant correlation between the volumes measured with radiological software iNtuition ${ }^{\circledR}$ and the volumes measured with ImageJ and Osirix ${ }^{\circledR}(r=0.93 ; r=0.95$, respectively). There were no major differences in total liver volumes, resection volumes, or tumour volumes for these three software packages.

Conclusions: Prospective hepatic CT-volumetry with ImageJ or OsiriX ${ }^{\circledR}$ is reliable and can be accurately used on a Personal Computer by non-radiologists. ImageJ and Osiri ${ }^{\circledR}$ yield result comparable to the radiological software iNtuition ${ }^{\circledR}$. 


\section{INTRODUCTION}

Hepatectomies are performed increasingly more often, mainly because indications for liver resection are continuously being extended. Whereas in the past a number of exclusion criteria were applied in the selection of patients for hepatectomy, they have now been largely abandoned and postoperative remnant liver volume and function have become the main determinants of resectability. ${ }^{1-3}$ Postoperative liver dysfunction may occur when the extent of tumour involvement requires major liver resection (3 or more segments), leaving a small postoperative remnant liver. ${ }^{4-6} \mathrm{~A}$ reliable volumetric assessment of the part of the liver to be resected and the future residual liver volume should be a critical part of preoperative evaluation particularly for extended resections, in order to prevent postoperative liver failure after liver resection. The safety of liver resection may increase if an estimate of minimal remnant liver volume is obtained via computed tomography (CT) volumetry. ${ }^{4,6-9}$ This is especially important if the underlying liver parenchyma is abnormal or has impaired functional capacity. In this context, repeated liver resections, increasingly extensive resections and resections after aggressive chemotherapy are likely to lead to more patients with small (functional) remnant livers and hence more risk of postoperative liver failure.

As the authors ${ }^{4,10-12}$ and others ${ }^{7,13,14}$ have shown before, liver volumes can be calculated accurately from CT or magnetic resonance imaging (MRI) scans. The liberal use of existing professional image-processing software is often limited by costs, access, flexibility, lack of radiologic staff and specific hardware requirements. In addition, the intended operation and the potential different surgical scenarios should be known to the investigator to predict the remnant liver volume accurately, which requires the expertise of a liver surgeon. The Digital Imaging and Communications in Medicine (DICOM) standard has enabled volumetry on a standalone or network attached Personal Computer (PC) remote from radiological hardware (CT- or MRI-scanner).

In 2007, our group conducted a retrospective study and proposed a method to perform liver volumetry with ImageJ, ${ }^{11}$ which is a free open-source image analysis software package developed at the National Institute of Health (NIH). ${ }^{8}$ In 2010, our group assessed the accuracy of OsiriX ${ }^{\circledR}$ for CT-volumetry of the liver. ${ }^{12}$ Osiri ${ }^{\circledR}$ is a more user-friendly, freely downloadable open source image analysis software package for Apple Mac OS. The accuracy for CT-volumetry of the liver resection specimen prospectively prior to surgery has not yet been assessed for either of these software packages. Therefore, the objective of the present study was to compare ImageJ and OsiriX ${ }^{\circledR}$ when performing prospective CT-volumetric analysis of the liver on a PC in patients undergoing major liver resection. The study also planned to compare the prospective measurements of ImageJ and Osirix ${ }^{\circledR}$ to those 
of CT volumetry performed by a radiologist using classic radiology software (iNtuition $^{\circledR}$ ) linked to a CT scanner system.

\section{METHODS}

\section{Patients}

Patients with primary or secondary liver tumours in otherwise normal livers (mostly colorectal cancer liver metastases) planned for a right hemihepatectomy at Maastricht University Medical Centre between January 2008 and April 2009 were eligible for inclusion in this study. All patients had a contrast enhanced CT scan in their routine preoperative assessment either in our hospital or in one of the surrounding university-affiliated district general teaching hospitals. Patients were admitted to the hospital one day preoperatively, and routine blood tests were performed.

\section{Surgical procedure}

Liver resection was performed as detailed elsewhere. ${ }^{15}$ In each patient, laparotomy was performed by bilateral subcostal incision, followed by intraoperative ultrasonographic (US) assessment of the liver. Once resectability had been confirmed, appropriate mobilization of the liver was performed as a prelude to hepatic parenchymal transection, which was undertaken using a Cavitron Ultrasonic Surgical Aspirator (Force GSU System; Valleylab, Boulder, CO, USA). Argon beam coagulation (Erbe, Tübingen, Germany), clips and sutures were used for haemostasis. Immediately after liver resection, weights of resection specimens were recorded in the operating theatre with a Mettler Toledo scale.

\section{CT volumetry}

For volumetric analysis, four-phase CT scans were used that were provided on CDRom on one of two different viewers: eFilm Lite (eFilm Medical,Toronto, Canada) or SIENET MagicView 300 VA42D (Siemens, Erlangen, Germany).

\section{Calculation of the liver volume using ImageJ}

ImageJ (version 1.33) was downloaded from http://rsb.info.nih.gov/ij/download.html (accession date: 01-01-08). The portovenous phase of all contrast enhanced CT-scans was used to facilitate optimal identification of liver segments and the anatomical resection plane. Details on how volumetry was performed with ImageJ have previously been described. ${ }^{11}$ Briefly, the relevant source images were individually copied $(\mathrm{Ctrl}+\mathrm{V})$ from the $\mathrm{CT}$ viewer on the electronic patient care system and opened with ImageJ by dragging them to the ImageJ main window in the appropriate order. In cases of tertiary referred patients relevant CT slices were searched in the original CT viewer on the CD-Rom. CT slices on a CD-ROM were 
usually saved as compressed DICOM-files, which cannot be opened or viewed via Windows Explorer. Therefore, the "Tudor DICOM Viewer Plug-in" was downloaded (free) from http://imagejdocu.tudor.lu/ and installed in the ImageJ menu. This plug-in allowed to preview the relevant CT files and open them directly in ImageJ.

The total liver, tumour and resected specimen were manually outlined. The respective region of interest (ROI) of each slice was added to the ROI manager. The area of each ROI was calculated and then multiplied by the slice thickness. Finally, all slice volumes were added up to calculate the volume of each three-dimensional structure (i.e., total liver resected specimen, tumour).

Prospective volumetric analysis: ImageJ

Volumetric analysis using ImageJ was performed prospectively by two nonradiologists (S.A.W.G.D., J.H.M.B.S.) during the preoperative evaluation of the patient and was blinded to the operating surgeon. For definition of the anatomic segments, Couinaud's classification was used. In all right hemihepatectomies, transection followed Cantlie's line from the top of the gallbladder, paralleling the middle hepatic vein straight to the supra hepatic inferior caval vein. In all patients the middle hepatic vein remained in situ with the liver remnant. Consequently, during the venous phase, the middle hepatic vein on CT was used to perform the volumetric measurement.

To assess the accuracy of volumetric measurements of the liver with ImageJ, the volumes of resected specimens were compared with actual weights of the specimens. (The weights of the specimens had been recorded in the operating theatre immediately after resection.) The actual weights of the resection specimens remained blinded to the investigators conducting CT-volumetry until all patients had been included and analysed prospectively. The functional remnant liver volume (FRLV) percentage was calculated with the following formula:

$$
F R L V[\%]=100 \% \times \frac{\text { total liver }- \text { resected volume }}{\text { total liver volume }- \text { tumour liver volume }}
$$

Prospective volumetric analysis: OsiriX ${ }^{\circledast}$

The 32-bit OsiriX ${ }^{\circledR}$ version 3.3 was downloaded from: http://www.osirixviewer.com. A 2.8-Ghz Intel Core 2 Duo 24" iMac (Apple, Cupertino, CA, USA) was used. Details on how volumetry was performed with Osiri ${ }^{\circledR}$ have been described previously. ${ }^{12}$ Volumes calculated with ImageJ were compared to the volumes obtained by CT-volumetry using OsiriX ${ }^{\circledR}$ by one of the liver surgeons (R.M.van D.).

\section{Comparison of ImageJ and OsiriX ${ }^{\circledR}$ with iNtuition ${ }^{\circledR}$}

Volumes calculated with ImageJ and OsiriX ${ }^{\circledR}$ were compared to the volumes obtained by CT-volumetry performed by a specialized liver radiologist (R.S.A. van S.) 
using radiology image analysis software from TeraRecon ${ }^{\circledR}$ linked to an Aquarius iNtuition ${ }^{\circledR}$ workstation. Volumetric analysis performed with ImageJ and OsiriX ${ }^{\circledR}$ was blinded to the radiologist. The volume of the liver resection specimen measured by the radiologist was used as a second standard of reference instead of the actual weight of the resection specimen.

\section{Reliability: ImageJ}

For analysis of interuser variability of ImageJ, volume measurements of total liver volume, functional liver volume and tumour volume performed before surgery by two investigators (S.A.W.G.D., J.H.M.B.S.) were compared. The first investigator had three years experience and the second investigator had no experience with the applied software and received instructions and training before starting with the measurements. Bland-Altman plots were used (see below, under Statisticical Analysis) to analyse the reliability of the ImageJ method.

\section{Time consumption: ImageJ}

The time consumed to perform one volumetric assessment of a liver with ImageJ including the total liver, the tumour, and the resected specimen was recorded for each of the 15 livers. To assess time consumption, workload and influence of slice thickness, the time needed to outline these three ROls (total liver, tumour, resected specimen) was recorded per slice in five livers.

As in any volumetric measurement technique based on CT imaging data sets, the accuracy of the volume measurements is dependent on the slice thickness used for the volume calculation. The slice thickness used for volume measurement is also crucial for the time needed to perform the analysis because of manual outlining. Therefore, the influence of slice thickness on CT- based liver volume measurements using ImageJ was also investigated. Total liver volume (TLV) was analyzed based on different slice thicknesses in these patients undergoing a right hemihepatectomy. The relative change in TLV between volumetry performed with the standard of reference $(0.5 \mathrm{~cm})$ and volumetric measurements using thicker slices $(1.0,1.5$, and $2.0 \mathrm{~cm})$ was calculated with the following formulas: $\left(\operatorname{TLV}_{1.0}-\mathrm{TLV}_{0.5}\right)$ / $\mathrm{TLV}_{0.5} \times 100 \%$; $\left(\mathrm{TLV}_{1.5}-\mathrm{TLV}_{0.5}\right) / \mathrm{TLV}_{0.5} \times 100 \% ;\left(\mathrm{TLV}_{2.0}-\mathrm{TLV}_{0.5}\right) / \mathrm{TLV}_{0.5} \times 100 \%$. The relative gain of time between volumetric measurements using different slice thicknesses was recorded and calculated in percentages as well.

\section{Statistical analysis}

Pearson's coefficient was used to quantify the association between the volume of the resected specimen and the actual weight of the resection specimen. A value of $P<0.05$ was considered to indicate statistical significance. The resulting regression line was described as a linear equation, and the correlation coefficient $(r)$ was cal- 
culated. Changes of calculated volumes were tested against zero with the use of a Wilcoxon signed rank test with a theoretical mean of zero. Data are presented as the mean \pm standard error of the mean (SEM).

The mean and standard deviation (SD) were calculated from the two volumetric results for each user using ImageJ. Next, the limits of agreement as proposed by Bland and Altman ${ }^{16}$ were calculated. This method is based on the mean and SD of the difference between two ratings of the same patient. The mean difference between two users indicates systematic error. The SD of the difference between two users indicates random error. The $95 \%$ limits of agreement as in Bland and Altman's plots result in both quantification of random error and systematic error (1.96* SD) of the measurement method. Statistics were performed using Prism 5.0 for Windows (GraphPad Software, San Diego, CA, USA).

\section{RESULTS}

\section{Patients}

Fifteen patients scheduled for right hemihepatectomy were included in the study. The median age of the patients was 63 years (48-79 years). None of the patients had preoperative evidence of underlying liver disease and routine liver tests were uniformly normal (table 1).

Table 1. Patient's characteristics

\begin{tabular}{ll}
\hline Characteristics & Median (range) \\
\hline Age (years) & $63(48-79)$ \\
Sex (M/F) & M 8, F 7 \\
Height $(\mathrm{cm})$ & $170(155-191)$ \\
Weight $(\mathrm{kg})$ & $76(55-127)$ \\
BMI $\left(\mathrm{kg} / \mathrm{m}^{2}\right)$ & $26(19-43)$ \\
AST $(\mathrm{IU} / \mathrm{I})$ & $22(7-52)$ \\
ALT $(I U / I)$ & $30(8-61)$ \\
LDH $(I U / I)$ & $372(312-469)$ \\
yGGT $(I U / I)$ & $37(29-258)$ \\
ALP $(I U / I)$ & $98(57-168)$ \\
Bilirubin $(\mu M)$ & $12(7-15)$ \\
\hline
\end{tabular}

$B M I$ body mass index, AST aspartate aminotransferase, $A L T$ alanine aminotrasferase, $L D H$ lactate dehydrogenase, $\gamma$ GGT $\gamma$-glutamyltranspeptidase, $A L P$ alkaline phosphatase

Relation between prospective CT-measured liver volume and resected liver weight The mean weight of the resection specimens measured in the operating theatre was 788.8 ( \pm 53.7$) \mathrm{g}$. The mean resected liver volumes calculated with ImageJ,

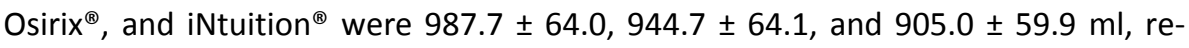


spectively. The mean ratios between measured weight and volume measured by ImageJ, Osirix ${ }^{\circledR}$ and iNtuition ${ }^{\circledR}$ was $0.78 \pm 0.03,0.81 \pm 0.04$, and $0.83 \pm 0.04$, respectively. The mean remnant liver volume percentages measured by ImageJ, Osirix ${ }^{\circledR}$ and iNtuition ${ }^{\circledR}$ was $41.4 \pm 1.6 \%, 36.2 \pm 1.5 \%$ and $37.8 \pm 1.6 \%$, respectively (Table 2 ). In one patient two surgical procedures were possible, either a right posterior sectionectomy or a right hemihepatectomy. Both volumes were calculated prospectively and finally the volume of right posterior sectionectomy was included in the analysis. There was a significant correlation between resected liver volumes calculated with ImageJ, Osirix ${ }^{\circledR}$ and iNtuition ${ }^{\circledR}$ prospectively and the actual measured weights of the resection specimens: $r=0.89$ and $P<0.001 ; r=0.83$ and $P<0.001$; $r=0.85$ and $P<0.001$; respectively (fig. 1 ).

Table 2. Results for 15 CT volumetry and intraoperative measurements

\begin{tabular}{lllll}
\hline Measurements & Image user 1 & ImageJ user 2 & OsiriX ${ }^{\circledR}$ & iNtuition $^{\circledR}$ \\
\hline Total liver volume $(\mathrm{ml})$ & $1736.0( \pm 104.0)$ & $1785.0( \pm 114.9)$ & $1536.0( \pm 98.6)$ & $1540.0( \pm 99.9)$ \\
Resected liver volume $(\mathrm{ml})$ & $987.7( \pm 64.0)$ & $1050( \pm 78.6)$ & $944.7( \pm 64.1)$ & $905.0( \pm 59.9)$ \\
Tumour volume $(\mathrm{ml})$ & $14.0( \pm 4.3)$ & $18.9( \pm 4.4)$ & $12.0( \pm 3.7)$ & $12.4( \pm 4.0)$ \\
Remnant liver volume $(\%)$ & $41.4( \pm 1.6)$ & $39.0( \pm 1.4)$ & $36.2( \pm 1.5)$ & $37.8( \pm 1.6)$ \\
Resected specimen weight / volume ratio $(\mathrm{g} / \mathrm{ml})$ & $0.78( \pm 0.03)$ & $0.77( \pm 0.03)$ & $0.81( \pm 0.04)$ & $0.83( \pm 0.04)$ \\
Resected specimen weight $(\mathrm{g})$ & $788.8( \pm 53.73)$ & & & \\
\hline
\end{tabular}

CT computed tomography

Interobserver variability: ImageJ

Liver volumetry measurements using ImageJ were compared using Bland-Altman plots. The mean difference of TLV measurements between both users was $49.4 \mathrm{ml}$ $\pm 86.1 \mathrm{ml}$. The $95 \%$ limits of agreement were -218.2 to $119.4 \mathrm{ml}$ (Fig. 2a). The mean difference of the liver resection specimen measurements between both users was $53.0 \mathrm{ml} \pm 89.8 \mathrm{ml}$. The $95 \%$ limits of agreement were -228.9 to $122.9 \mathrm{ml}$ (Fig. 2b). The mean difference of the \%FRLV measurements between both users was $2.3 \% \pm$ 4.6\%. The $95 \%$ limits of agreement were $-6.7 \%$ to $11.4 \%$ (Fig. 2 c). 


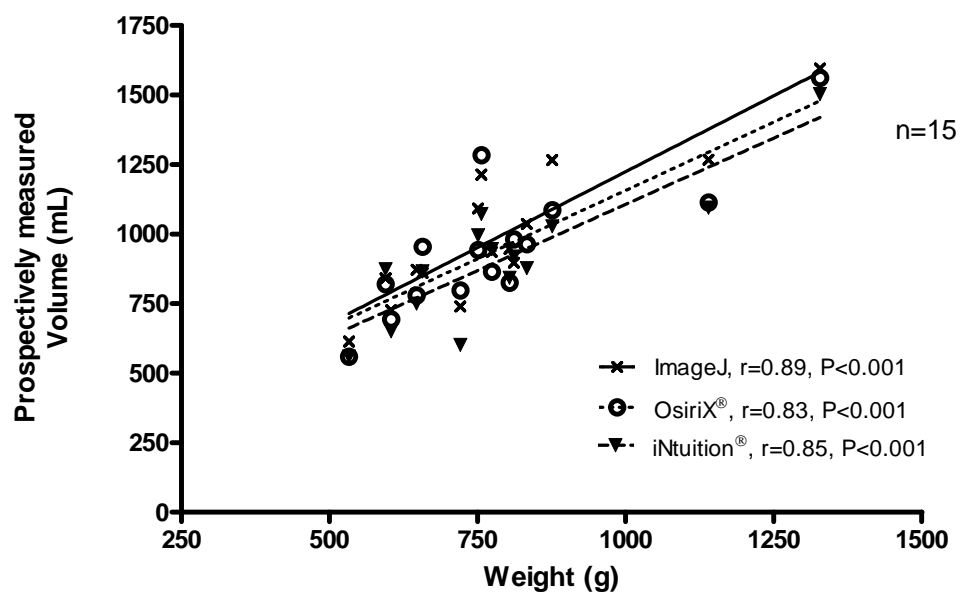

Figure 1. Correlation between volume measured prospectively with ImageJ, Osirix ${ }^{\circledR}$ and iNtuition ${ }^{\circledR}$ and the resected specimen's weight measured in the operating theatre.

\section{Total Liver Volume}

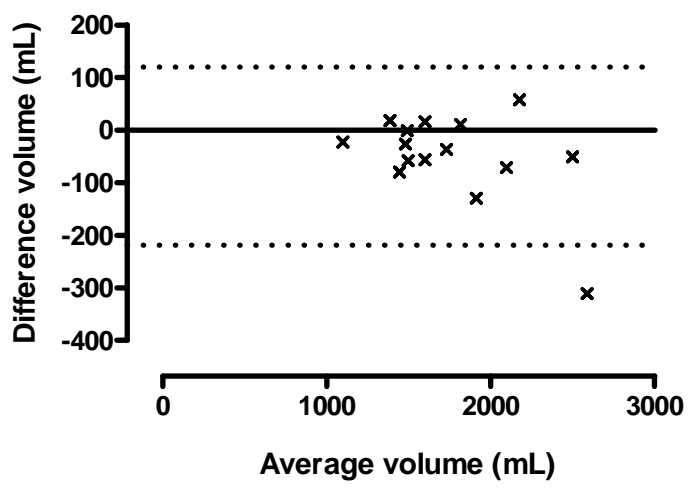

Figure 2a. Bland-Altman analysis for interobserver variability of outlining the total liver volume with ImageJ. Horizontal solid line indicates the mean average between the 2 users. Horizontal dashed lines indicate $95 \%$ limits of agreement (mean $\pm 1.96 \mathrm{SDs}$ ). 


\section{Resection Volume}

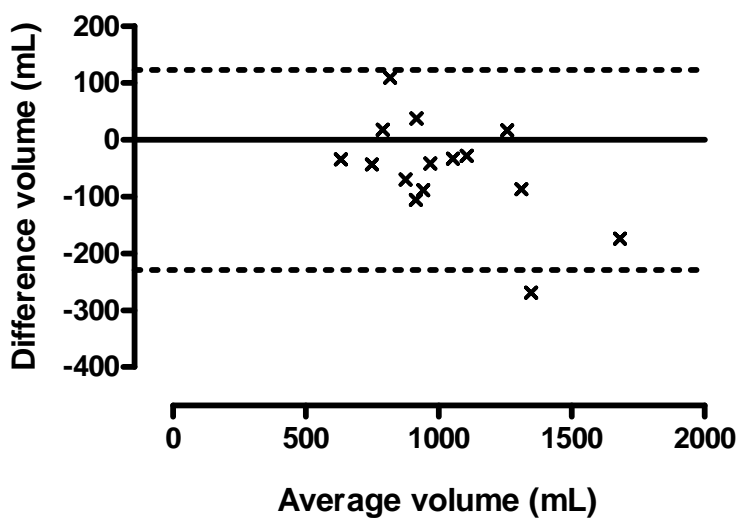

Figure 2b. Bland-Altman analysis for interobserver variability of outlining the resectional liver volume with ImageJ.

\section{\%FRLV}

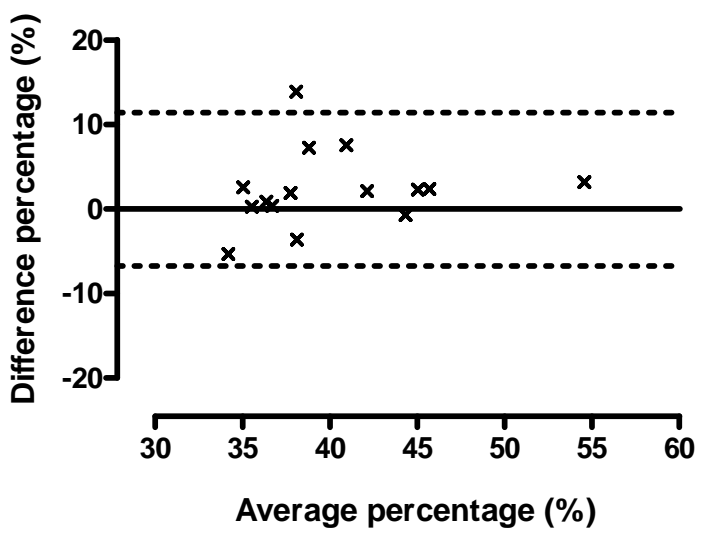

Figure 2c. Bland-Altman analysis for interobserver variability of the \%functional remnant liver volume (\%FRLV) with ImageJ.

Time to perform measurement and effect of slice thickness

Time required for a single volumetric assessment of the liver with ImageJ (including total liver, tumour, resected specimen) was, on average, 20 minutes (range 15-30 minutes). There was no statistical difference between the TLVs based on 0.5 vs. 1.0 $\mathrm{cm}, 0.5 \mathrm{vs} .1 .5 \mathrm{~cm}$, and $0.5 \mathrm{vs} 2.0 \mathrm{~cm}$. The change in volume (calculated in percentages) was not significantly different for volumetric measurements with different slice thicknesses. For a slice thickness of 1.0 or $1.5 \mathrm{~cm}, 80 \%$ of the measurements were within the $5 \%$ limit of acceptance for the TLV change. When using the $2.0 \mathrm{~cm}$ slice thickness, only $60 \%$ of the measurements were within this limit. The mean 
gain of time (calculated in percentages) by increasing slice thickness was $50 \%(0.5$ vs $1.0 \mathrm{~cm}$ ), $64 \%$ (0.5 vs $1.5 \mathrm{~cm}$ ) and $72 \%$ (0.5 vs $2.0 \mathrm{~cm})$.

\section{ImageJ versus Osiri $X^{\circledR}$ and iNtuition ${ }^{\circledR}$}

There was a highly significant correlation between the volumes calculated with ImageJ and the volumetric analysis using $O$ siri $X^{\circledR}(r=0.95, P<0.001)$. There was also a strong correlation between the volumes calculated with ImageJ and the volumetric analysis performed by a radiologist using radiology image analysis software linked to radiological hardware $(r=0.93, P<0.001)$.

\section{DISCUSSION}

The present study shows that prospective CT volumetric analysis of the liver in patients undergoing right hemihepatectomy with ImageJ and Osirix ${ }^{\circledast}$ on the surgeon's PC is valid and reliable. In this study, we found a strong significant correlation between resection weight and resection volume measured prospectively with ImageJ and Osiri $X^{\circledR}$. Practical advantages of ImageJ and Osirix ${ }^{\circledR}$ were described previously by our group. ${ }^{11,12,17}$ In our centre, the radiologists use the commercial image analysis software package iNtuition ${ }^{\circledR}$ for liver CT volumetry. There was a significant correlation between the resection weight and the resection volume measured prospectively with iNtuition ${ }^{\circledR}$. There were no significant differences in the mean volumes between the three software packages. There was a strong correlation between the volumes measured with radiology software iNtuition ${ }^{\circledR}$ and the volumes measured with ImageJ and Osirix ${ }^{\circledR}$. This indicates that ImageJ and Osirix ${ }^{\circledR}$ even if operated by nonradiologists, are at least as appropriate for CT volumetry of the liver as professional radiology software. ImageJ and Osirix ${ }^{\circledR}$ bring preoperative liver CT volumetry within the surgeon's reach, independent of the support of a radiologist. Hepatopancreatobiliary (HPB) surgeons should routinely perform CT volumetry in the preoperative assessment of patients undergoing a major liver resection. This is especially true for patients with a compromised liver function due to neoadjuvant chemotherapy, cirrhosis or steatosis or patients scheduled for an extended liver resection.

As we $\mathrm{e}^{11}$ and others ${ }^{18}$ concluded before, the time required to perform CT volumetry of the liver can be reduced by increasing slice thickness and thereby diminishing the number of CT slices that have to be outlined. Data from the current study show that in order to make a rough estimate of the FRLV (patients with a percentage FRLV of $\geq 30 \%$ ) a slice thickness of $1.0 \mathrm{~cm}$ provides an optimal balance between accuracy and time efficiency. However, in patients with a marginally functional liver remnant volume $(20-30 \%)$ a greater accuracy is needed. In these patients it is better to use a smaller slice thickness in order to avoid over- or underestimation of the 
volume. Time required to outline all ROIs is approximately 20 minutes in both ImageJ and OsiriX ${ }^{\circledR}$. However, OsiriX ${ }^{\circledR}$ has a significant advantage because it has an integrated picture archiving and communication system (PACS-system) in which the data (CT scans) of all examined patients are automatically stored. Additionally, OsiriX ${ }^{\circledR}$ is more user-friendly and ROIs can be selected semi-automatically, which saves time and lessens workload. ${ }^{12}$

Interobserver variability for ImageJ between the two users for the volumetric assessment of the total and the resection liver volumes was low. Bland-Altman plots revealed that there was one outperformer in the measurements of the total liver volume and the resection specimen volume. This resulted in relatively wide confidence intervals. However, it is mainly the \%FRLV that is crucial. It has been shown elsewhere that to perform liver resection safely \%FRLV has to be at least $26.6 \%$ in patients with normal liver parenchyma. ${ }^{4}$ In the present study, the mean difference of the \%FRLV measurements between both ImageJ users of $2.3 \% \pm 4.6 \%$ was clinically acceptable because ImageJ users 1 and 2 predicted a \%FRLVs of 41.4 $\pm 1.6 \%$ and $39.0 \pm 1.4 \%$, respectively. In other words, the resection volume differences between the two ImageJ users should have had no clinical consequences. In patients in whom the preoperative liver volume assessment shows that the reported FRLV\% approaches $26.6 \%$, extra care is recommended.

A possible limitation of estimating the future remnant liver volume with CT volumetry is the systematic overestimation of the liver volume compared to resection specimens' weight. The physical density $<1.0 \mathrm{~g} / \mathrm{ml}$ and the systematic overestimation of the liver volume in the present study was in accordance with previous studies. ${ }^{7,10-12,}$ Inaccurate estimation of the resected specimen volume by CT volumetry can occur because the liver on a CT scan is in a perfused state and the postoperative resection specimen is in an unperfused state. This applies to every radiologic application, and therefore some studies suggested the implementation of conversion factors to improve volumetric accuracy. ${ }^{7,19,20}$ These factors are mostly based on the intraoperative resection volume determined with the use of the Archimedes principle. Unfortunately, this approach does not determine the influence of the blood content of the resection specimen. It might be that these conversion factors can lead to a more precise volumetric analysis of the liver, but their exact role remains to be determined. Patients with steatosis have more fat in the liver, and the relative density of fat is $0.9 \mathrm{~g} / \mathrm{ml}$. Hence, the volume is larger and the weight less than that of a normal liver. Ultimately this changes the volume/weight standard and can also contribute to this systemic overestimation.

In all right hemihepatectomies in the present study, virtual transection followed Cantlie's line and thus the middle hepatic vein (MHV) was used as a guide for prospective volume measurements. During surgery the demarcation line after selective inflow occlussion is used for transection and may leave liver parenchyma outside (i.e. to the right of) the MHV in some patients. As a consequence, preop- 
erative estimation of the future resected specimen volume may be overestimated in some individuals. In line with this, Karlo et al. showed in a retrospective study that, despite a highly accurate definition of the transection line on preoperative CT or MRI scans using anatomical landmarks on postoperative MRI scans, the resection specimen volume was still overestimated. ${ }^{7}$ Systematic overestimation, however, does not affect the functional remnant liver volume as a percentage of the total functional liver volume, which is one of the most important indicators of the risk of postoperative liver failure.

\section{Conclusions}

User-friendly, easily accessible, free, downloadable software packages such as ImageJ and OsiriX ${ }^{\circledR}$ can be used reliably for prospective CT volumetry of resected liver specimens. The performance of ImageJ and OsiriX ${ }^{\circledR}$ is comparable to that of radiology software iNtuition ${ }^{\circledR}$. OsiriX ${ }^{\circledR}$ is the most efficient and practical software package, but it can be used only at a Macintosh PC. For a Windows-based PC, ImageJ is a good alternative.

Acknowledgements The authors thank M.H.A. Bemelmans, S.W.M. Olde Damink and M.C.G. van de Poll, for their contributions to conception and design of the study and for technical assistance. S.A.W.G. Dello was supported by a Kootstra Talent Fellowship from Maastricht University.

\section{REFERENCES}

1. Shah SA, Haddad R, Al-Sukhni W, Kim RD, Greig PD, Grant DR, et al. Surgical resection of hepatic and pulmonary metastases from colorectal carcinoma. J Am Coll Surg. 2006;202:468-75.

2. Fusai G, Davidson BR. Management of colorectal liver metastases. Colorectal Dis. 2003;5:2-23.

3. Scheele J, Altendorf-Hofmann A, Grube T, Hohenberger W, Stangl R, Schmidt K. [Resection of colorectal liver metastases. What prognostic factors determine patient selection?]. Chirurg. 2001;72:547-60.

4. Schindl MJ, Redhead DN, Fearon KC, Garden OJ, Wigmore SJ. The value of residual liver volume as a predictor of hepatic dysfunction and infection after major liver resection. Gut. 2005;54:289-96.

5. Shoup M, Gonen M, D'Angelica M, Jarnagin WR, DeMatteo RP, Schwartz LH, et al. Volumetric analysis predicts hepatic dysfunction in patients undergoing major liver resection. J Gastrointest Surg. 2003;7:325-30.

6. Jarnagin WR, Gonen M, Fong Y, DeMatteo RP, Ben-Porat L, Little S, et al. Improvement in perioperative outcome after hepatic resection: analysis of 1,803 consecutive cases over the past decade. Ann Surg. 2002;236:397-407.

7. Karlo C, Reiner CS, Stolzmann P, Breitenstein S, Marincek B, Weishaupt D, et al. CT- and MRI-based volumetry of resected liver specimen: Comparison to intraoperative volume and weight measurements and calculation of conversion factors. Eur J Radiol. 2010;75:107-111.

8. Rasband W. In: ImageJ, 1.33 edn. National institutes of Health. Maryland, USA 1997-2005 


\section{Chapter 13}

9. Kishi Y, Abdalla EK, Chun YS, Zorzi D, Madoff DC, Wallace MJ, et al. Three Hundred and One Consecutive Extended Right Hepatectomies: Evaluation of Outcome Based on Systematic Liver Volumetry. Ann Surg. 2009;250(4):540-8

10. Wigmore SJ, Redhead DN, Yan XJ, Casey J, Madhavan K, Dejong CH, et al. Virtual hepatic resection using three-dimensional reconstruction of helical computed tomography angioportograms. Ann Surg. 2001;233:221-226.

11. Dello SA, van Dam RM, Slangen JJ, van de Poll MC, Bemelmans MH, Greve JW, et al. Liver volumetry plug and play: do it yourself with ImageJ. World J Surg. 2007;31:2215-2221.

12. van der Vorst JR, van Dam RM, van Stiphout RS, van den Broek MA, Hollander IH, Kessels AG, et al. Virtual liver resection and volumetric analysis of the future liver remnant using open source image processing software. World J Surg.2010;34:2426-2433.

13. Lu Y, Wu Z, Liu C, Wang HH. Hepatic volumetry with PhotoShop in personal computer. Hepatobiliary Pancreat Dis Int. 2004;3:82-85.

14. Tu R, Xia LP, Yu AL, Wu L. Assessment of hepatic functional reserve by cirrhosis grading and liver volume measurement using CT. World J Gastroenterol. 2007;13:3956-3961.

15. Dejong CHC, Garden OJ. Neoplasms of the liver. In: Majid AA Kingsnorth A, (eds) Advanced surgical practice. London: Greenwich medical Media 2003;146156.

16. Bland JM, Altman DG. Comparing methods of measurement: why plotting difference against standard method is misleading. Lancet. 1995;346:1085-1087.

17. van der Vorst J, van Dam R, van Stiphout $R$, Hollander I, van den Broek M, Kessels F, et al. Virtual liver resection and volumetric analysis of the future liver remnant using open source image processing software. World J of Surg. 2010;34(10):2426-2433.

18. Reiner CS, Karlo C, Petrowsky H, Marincek B, Weishaupt D, Frauenfelder T. Preoperative liver volumetry: how does the slice thickness influence the multidetector computed tomography- and magnetic resonance-liver volume measurements? J Comput Assist Tomogr. 2009;33:390-397.

19. Lemke AJ, Brinkmann MJ, Schott T, Niehues SM, Settmacher U, Neuhaus $P$, et al. Living donor right liver lobes: preoperative CT volumetric measurement for calculation of intraoperative weight and volume. Radiology. 2006;240:736-742.

20. Hwang S, Lee SG, Kim KH, Park KM, Ahn CS, Moon DB, et al. Correlation of blood-free graft weight and volumetric graft volume by an analysis of blood content in living donor liver grafts. Transplant Proc. 2002;34:3293-3294. 


\section{Chapter 14}

\section{Select|ve arterial emboLisation VErsus}

Resection of hepatocellular adenomas

(SILVER-trial): Study protocol for a

randomized controlled trial

M.C. de Jong, J.H.M.B. Stoot, R.J.S. Coelen, R.M. van Dam, M.G. Besselink, M.W. de Haan, M.A.A.J. van den Bosch, C.H.C. Dejong

On behalf of the Dutch Liver Surgery Study Group:

C. Verhoef, T.M. van Gulik, R.J. Porte, J.N.M. IJzermans, A.F. Schaapherder, J.H.W. de Wilt, R. van Hillegersberg, J. Klaase, R.M.H. Roumen, A.M. Rijken, M.S.L. Liem

Presented to the European-African Hepato-Pancreato-Biliary Association in Cape Town, South Africa, April 2011. 


\section{ABSTRACT}

Background: Hepatocellular adenomas (HCAs) are rare benign tumours mostly occurring in women taking oral contraceptives. Due to the risk of rupture and malignant transformation, resection is suggested for HCAs $>5 \mathrm{~cm}$. Recent studies showed that selective arterial embolisation of haemorrhaging and nonhaemorrhaging HCAs was effective not only as acute treatment but, moreover, also decreased the size of the HCAs. Recently, due to genetic screening, it has also become possible to identify adenomas with a very low to negligible risk of malignant transformation. A pilot study performed at the MUMC+ demonstrated the feasilibity of arterial embolisation to treat HCA.

The aim of the current study is to test the hypothesis that minimally invasive embolisation of non-ruptured HCAs leads to a reduction of the post-treatment morbidity (i.e. any event with Clavien-Dindo $\geq 3$ ) and mortality compared with a resectional approach.

Methods/design: a randomised controlled trial with a two-arm experimental design. Following a protected fine needle biopsy, patients with a negative $\beta$-catenin status and non-elevated $\alpha$-fetoprotein (AFP) levels who are eligible for resection of their HCA will be randomised to either resection or selective arterial embolisation. The primary endpoint is the proportion of patients with treatment-related morbidity and mortality (within 90 days after randomization). Secondary outcome parameters are functional recovery, quality of life and post-treatment body-image changes as well as direct and indirect medical costs. A blinded adjudication committee will judge all endpoints. Patients will be followed for 5 years with annual serum AFP levels and MRI of the liver follow up to screen for malignant transformation.

Discussion: The current trial is a randomised controlled multicentre trial that will provide evidence on the merits of selective arterial embolisation of HCA.

Registration: the trial will be registered with ClinicalTrials.gov. 


\section{INTRODUCTION}

Hepatocellular adenoma (HCA) is the most important, albeit uncommon, benign tumour of the liver. Although the reported incidence approximated one per million during the 1970s, a rise in incidence is seen over the last few decades. ${ }^{1,2}$ This is believed to be strongly associated to the widespread use of oral contraceptives, ${ }^{3-6}$ as the incidence of these HCAs in long-term users of oral contraceptives approaches 30-40 per million. ${ }^{1,2}$ Other, less common aetiologies include use of anabolic androgens and history of glycogen storage disease. ${ }^{7-11}$

HCAs are hypervascular lesions comprised of multiple sinusoids of dilated thinwalled capillaries with exclusively arterial blood supply, thereby resulting in a high blood pressure within these tumours. ${ }^{12}$ Although the majority of lesions are incidental findings on cross-sectional images of the liver for unrelated reasons, some patients present with life-threatening haemorrhage. ${ }^{3,5}$ It is this risk of spontaneous bleeding, ranging from $20-40 \%,{ }^{13-15}$ that may require treatment of HCAs even in absence of symptoms. Conflicting data regarding risk factors for severe haemorrhage is available, as some authors suggest that volume of the tumour and number of lesions pose no threat of bleeding. ${ }^{16,17}$ Others claim that the risk for spontaneous bleeding is related to the size of the HCA, since intratumoural bleeding is more frequently observed in large lesions $(>5 \mathrm{~cm})$, and possibly due to the use of hormones. ${ }^{18-23}$ Furthermore, a considerable body of literature is available to support a potential for malignant evolvement of this benign process into hepatocellular carcinoma ( $\mathrm{HCC}$ ), with a substantial risk of $4.3 \% .{ }^{24}$ An established risk factor for this transformation to a malignant neoplasm is the size of the HCA, as malignant evolvement is rare in lesions smaller than $5 \mathrm{~cm} .{ }^{12,18,25}$ As an additional risk factor, it has been suggested that a positive $\beta$-catenin status is associated with an increased chance of malignant alteration, although the majority of HCAs have a negative $\beta$ catenin status. ${ }^{26}$ The $\mathrm{Wnt} / \beta$-catenin signaling pathway plays a role within hepatic development, growth and regeneration, hence an aberrant, positive status has been shown to increase the risk of development of HCC. ${ }^{27,28}$

Thus far, elective surgical resection has been regarded the gold standard treatment for patients with a HCA, as it provides long-term cure of symptoms, eradicates the possibility of bleeding and malignant evolvement and eliminates the option of leaving a lesion in situ that is misclassified on imaging as being benign. ${ }^{12,16,19,21}$ Especially in the group of lesions equal to or larger than $5 \mathrm{~cm}$, resection is advised based on the previously mentioned risks of spontaneous bleeding and malignant transformation. ${ }^{18,20,21,23}$ 
In case of a haemorrhaging HCA, acute partial liver resection is associated with high perioperative morbidity and perioperative mortality. ${ }^{29,30}$ However, recent studies $^{12,31-33}$ found selective arterial embolisation for ruptured and haemorrhaging HCA to be safe and effective in realizing haemodynamic stability. Moreover, a significant reduction in size was found on computed tomography (CT scan) or magnetic resonance imaging (MRI scan) after embolisation, ${ }^{32}$ probably caused by the hypervascular nature of the HCAs with its exclusive arterial blood supply. ${ }^{12,32,34}$ It is this tumour regression in both haemorrhaging and non-haemorrhaging $\mathrm{HCAs},{ }^{32,34}$ and its subsequent reduction of the risk of severe haemorrhaging and probably also malignant transformation, which supports the use of selective arterial embolisation as a treatment for unruptured HCAs. Furthermore, even though elective liver resection for a non-ruptured benign tumour is associated with a low perioperative morbidity and near zero perioperative mortality, ${ }^{13,17,20,35}$ selective arterial embolisation is hypothesized to be a less invasive treatment for patients with a HCA, of whom the majority is represented by young, otherwise healthy individuals. Regarding the results of previous non-randomised studies and case series, it appears that selective embolisation for HCAs is an effective treatment for haemorrhaging and nonhaemorrhaging HCAs. We therefore seek to compare the safety and efficacy of selective arterial embolisation to surgical resection for non-haemorrhaging HCAs larger than $5 \mathrm{~cm}$ in diameter, to determine the most favourable treatment for these benign liver tumours.

\section{METHODS}

\section{Study objectives}

The aim of the current study is to determine whether selective arterial embolisation for non-haemorrhaging HCAs is to be preferred over surgical resection of the lesion. Two research questions are employed in order to answer this objective. First, how does selective arterial embolisation compare to surgical resection in terms of perioperative morbidity and mortality (i.e. any event with a Clavien-Dindo classification Grade $\geq 3$ ) within 90 days of treatment. Secondly, how do these two treatments compare with regard to long-term outcome such as quality of life and post-treatment body-image changes, functional recovery and direct medical and non-medical costs?

\section{Design of the study}

The current study is a multicentre, parallel-group, randomised trial to determine whether selective arterial embolisation is to be preferred over resection in patients 
with an HCA measuring more than $5 \mathrm{~cm}$ in diameter. Data will be analysed according to the intention to treat principle. Randomisation is performed by an internet randomisation module; block-randomisation per centre is used. Therefore, this design results in two treatment groups: patients who are randomised to selective arterial embolisation (group I) and patients who are randomised to surgical resection of the HCA (group II) (the choice of operative approach (i.e. an open versus laparoscopic approach) can be made at the discretion of the individual attending surgeon). (figure 1).

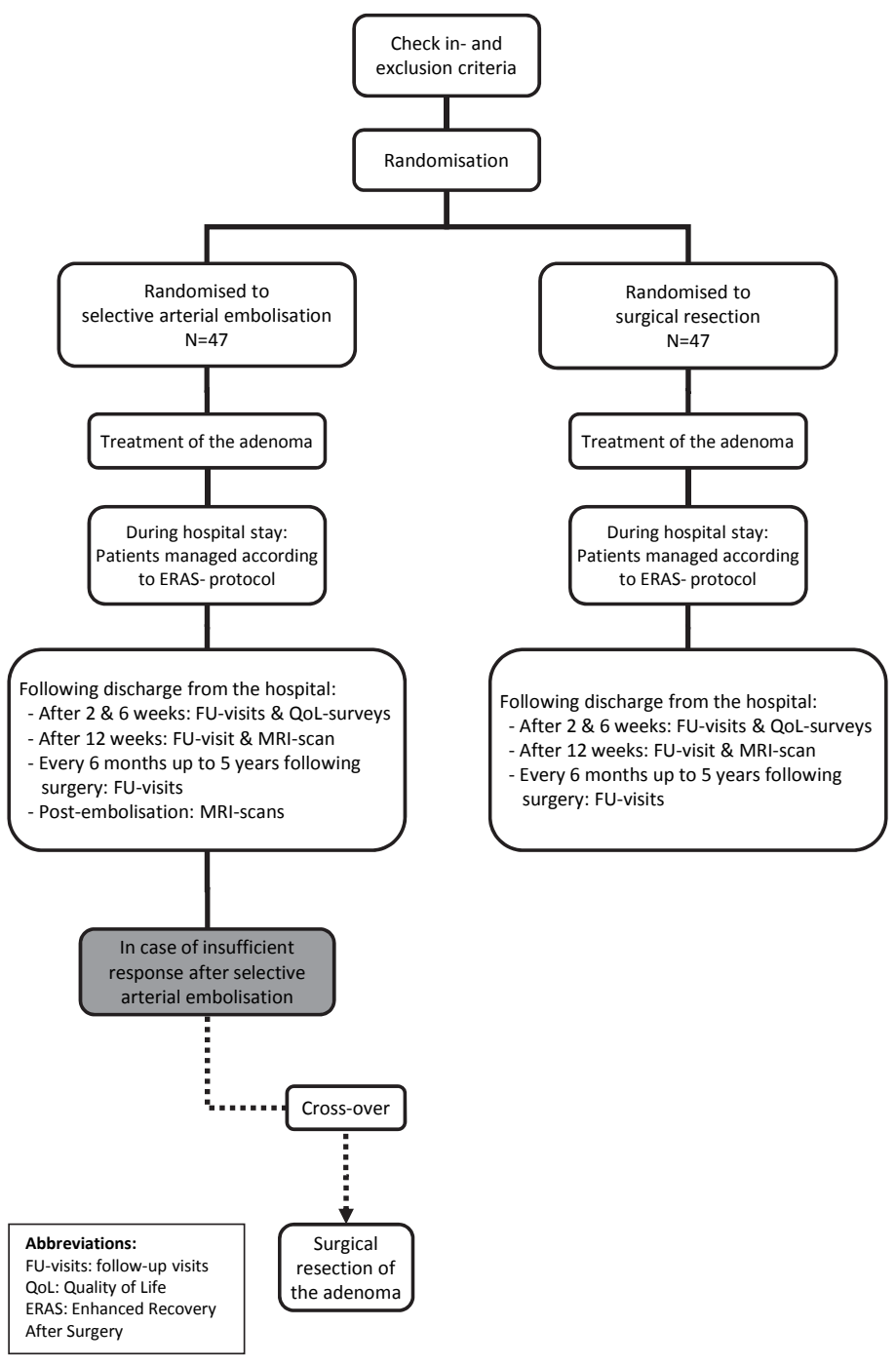

Figure 1. Flowchart 
Moreover, it is imperative to be able to follow-up patients who did not meet the inclusion criteria of the current trial, as the absence of these patients may restrict generalisation of the results thus causing reduced external validity, which might compromise the representativeness of the study population. Due to unknown and uncontrolled confounders, these patients will be enlisted and subsequently analysed in a supplementary observational study.

If signs of malignancy are detected on pre-inclusion MRI scan or CT scan or on a protected fine needle biopsy, the patient will be excluded from this trial and will receive standard treatment for (suspected) HCC. Specifically, all patients will undergo a protected fine needle aspiration in order to determine the $\beta$-catenin status of their HCA. In addition to the aforementioned pro-malignant properties of $\beta$ catenin, it has been hypothesized that inducing hypoxia in $\beta$-catenin positive tumours may be associated with a higher risk of invasive growth and development of distant metastases. ${ }^{36}$ It is therefore of particular importance to determine the $\beta$ catenin status of the HCA before hypoxia is induced by means of selective arterial embolisation. Moreover, $\alpha$-fetoprotein (AFP) has been established as a marker for the diagnosis of HCC and therefore the serum levels of AFP will be evaluated prior to inclusion in the current trial. Those patients in whom an elevated level of AFP is found, will not be considered for participation in the SILVER-trial.

The success of the therapy will also be measured by the degree of cross-over, specifically the number of patients who eventually underwent resection of the HCA as a result of insufficient response after selective arterial embolisation. Insufficient response, based on the series of Stoot et al, ${ }^{32}$ is defined as less than $25 \%$ reduction in size within 24 months after embolisation, growth of the tumour, persisting or emerging symptoms or haemorrhage. Therefore, success of therapy is defined as cross-over of less than $20 \%$.

\section{Primary and secondary endpoints}

The aim of the current study is to test the hypothesis that minimally invasive, i.e. transarterial embolisation of non-ruptured HCAs will lead to a reduction of the post-treatment morbidity and mortality (i.e. any event that required a therapeutic intervention or hospitalisation) compared with a resectional approach. The primary endpoint is the proportion of patients with treatment-related morbidity and mortality, with Clavien-Dindo Grade $(\geq 3) .{ }^{37}$ Therefore, adverse events occurring from the time of the intervention until 90 days after discharge from the hospital are compared. 


\section{Morbidity}

Overall, compared with surgical resection, selective arterial embolisation is a safe procedure and a minimally invasive alternative. Post-embolisation morbidity is mostly self-limiting and may include fever; conversely, complications requiring reinterventions have also been reported. ${ }^{32}$

Peri and post-operative morbidity could encompass haemorrhagic complications that occur predominantly during surgery or in the early postoperative phase, while biliary complications, intra-abdominal abscess or even liver failure may occur at a later phase. Also wound infection and sepsis will be monitored. Morbidity will be classified and analysed according to the validated classification for postoperative morbidity as described by Clavien-Dindo. ${ }^{37}$

Secondary outcome parameters are functional recovery, quality of life and posttreatment body-image changes (Short Form 36 (SF-36) and the gastrointestinal Quality of Life Index (GIQLI)), and direct and indirect medical costs. Specifically, patients will be followed over time, thereby providing data on the overall effectiveness of selective arterial embolisation compared with resection and thus giving insight in possible long-term adverse events.

\section{Post-treatment functional recovery}

Post-operative functional recovery is measured in days and length of posttreatment hospital stay (measured in days and including re-admittance rate). Functional recovery is based on several criteria.

Firstly, adequate pain control with oral analgetics needs to be accomplished. Post-operative pain will be systematically registered twice daily using the validated verbally administered 0-10 NRS-11 (11 points numeric rating scale). ${ }^{38-40}$ Members of a specialised pain team will inquire patients to rate the intensity of their current pain on a scale of 0 ("no pain") to 10 ("worst possible pain"). A score of 1-3 will be considered to be mild, 4 to 6 to be moderate and 7 to 10 to be severe. ${ }^{41}$

Secondly, mobility at the preoperative level must be achieved. To report the differences between pre- and post-operative level of mobility, we developed the 'ERAS Mobility scale' (EMS), which is inferred from the 'Groningen activity restriction scale'. ${ }^{42}$ To compare the level of mobility before and after surgical intervention, the EMS, which contains nine items of basic actions, is used. Patients have to report their ability to fully independently perform these actions. This assessment will be performed once preoperatively and daily during post-operative admission, until mobility at the preoperative level is achieved. [Appendix 1].

Furthermore, patients must be able to eat solid foods, must have a normal or decreasing serum bilirubin and should no longer be dependent on intravenous 
fluids. In general, when the criteria for full functional recovery are met, it is medically justified to discharge a patient. However, despite pre-admission counselling, an average delay in discharge of two days is reported, counting from the day full functional criteria are reached. ${ }^{43,44}$ Frequent causes of this delay are social problems, difficulties concerning homecare support or transportation issues. Therefore the data on functional recovery criteria and on the hospital length of stay will be independently collected and analysed.

\section{Quality of life and post-treatment body-image changes}

Differences in quality of life and changes in post-treatment body-image will be assessed for patients who underwent selective arterial embolisation compared with patients who underwent liver resection. We will use the Dutch language version of the "Short Form 36-items" (SF-36) health status test in Dutch centres. The standard SF-36 will be used in international centres. The SF-36 is a well-validated, short-form health survey comprised of 36 questions. ${ }^{45,46}$ It yields an 8 -scale profile of functional health and well-being scores as well as psychometrically-based physical and mental health summary measures and a preference-based health utility index. Assessment of quality of life will be performed at the time of consent, discharge and two and six weeks after discharge [Appendix 2, in Dutch].

\section{Hospital costs and economic evaluation}

The economic evaluation will include a cost-utility analysis from a societal perspective. The incremental costs per Quality Adjusted Life Year (QALY) gained will be based on utility scores from the SF-36. ${ }^{47,48}$ All hospital costs (direct and indirect) related to both interventions will be assessed. In addition, costs related to activities or resources outside health care will be measured using a cost questionnaire. Unit prices will be based either on prices from the hospital financial department or the Dutch guidelines for cost-calculation. ${ }^{48}$ Moreover, the impact on productivity will be assesed, using the PRODISQ questionnaire (PRODuctivity and DISease Questionnaire [appendix 3, in Dutch]). The time horizon of the economic evaluation will be 24 months (similar to the clinical study).

\section{Long-term adverse events}

While embolisation is hypothesized to be a safer procedure on the short-term, the current trial will also provide particular insight in the long-term effectiveness of this treatment compared with resection. Possible long-term adverse events, especially following hepatic resection, include incisional hernia, ileus or pain and occurrence of these complications will be recorded. Moreover, particularly in the post- 
embolisation group an important question which will be addressed, is whether these patients are still at risk for developing the aforementioned HCA-associated complications of hemorrhaging and malignant alteration. Therefore the rate of these specific adverse events will be noted and subsequently analysed.

\section{Participating centres}

All major hepato-pancreato-biliary (HPB) centres in the Netherlands will participate in this study.

\section{Study population}

Inclusion criteria

- HCA measuring at least 5 centimetres in diameter

- Patients fit to undergo liver resection

- Patients understands both the nature and requirements of the study

- Age at least 18 years

- BMI between 18-35

- ASA I-III

- Oral and written informed consent

\section{Exclusion criteria}

- Inability to give written informed consent

- HCA presenting with acute bleeding

- History of hepatic malignancy

- Adenomatosis (>10 HCAs)

- Presence or strong suspicion of HCC, determined on MRI scan or CT scan

- Positive positive $\beta$-catenin status determined after fine needle biopsy

- Increased level of AFP

- Pregnancy

- ASA IV-V

\section{Ethics}

The current study will be conducted in accordance with the principles of the revised version of the Declaration of Helsinki (October 2008, Seoul). The study protocol will be approved by the independent medical ethics committees of the participating hospitals. Written informed consent will be obtained from all patients prior to randomisation. 


\section{Safety and quality control}

All procedures will be performed by experienced hepatic surgeons and interventional radiologists. Every six months a study group meeting will be organized, which is aimed at discussing all newly randomised patients. In particular, an independent monitoring-committee, consisting of two hepatic surgeons, an interventional radiologist and an epidemiologist, will discuss all major adverse events (all morbidity Clavien-Dindo Grade $\geq 3^{37}$ or signs of malignant alteration after treatment). This monitoring-committee will subsequently give advice to the trial steering committee.

\section{Study outline}

Patients who fulfil the inclusion criteria will be recruited at the outpatient clinic. Subsequently, following one week of reflection, written informed consent will be obtained.

The randomisation will be done through the trial website. The experimental design produces two treatment groups: patients randomised to selective arterial embolisation (I) and patients randomised to surgical resection of their HCA (II). (figure 1). Analyses of the unrandomised groups will be treated as observational studies. $^{49}$

Before inclusion in this trial, an MRI scan or CT scan and, as previously explained, a protected fine needle biopsy ${ }^{50,51}$ will be performed. In case of findings suggestive for malignancy either on imaging ${ }^{52-55}$ or on biopsy, ${ }^{26,56,57}$ and moreover, if the HCA is found to have a positive $\beta$-catenin status, the patient will be excluded from participating in this trial and treated according to the current guidelines for hepatic malignancies. Furthermore, if patients are found to have an elevated level of AFP, patients will not be eligible for participating in the current trial and will receive treatment tailored at their possible malignancy. All patients will have a standard pre-assessment by the anaesthesiologist.

\section{Surgery}

Resection of the HCA is undertaken at the discretion of the attending surgeon, both the open and laparoscopic approach are permitted.

\section{Selective arterial embolisation}

Selective arterial embolisation is performed according to the technique applied by the local interventional radiologist. 


\section{Perioperative care programme: Enhanced Recovery After Surgery (ERAS)}

Preferably, all patients will be managed according to the ERAS-protocol (Enhanced Recovery After Surgery). ${ }^{58}$ Daily guidelines of the pre- and post-operative care of patients undergoing a liver resection according to the ERAS programme are depicted in appendix 3 in order to establish a standardized pre- and post-operative management.

This multimodal ERAS programme has proven to significantly accelerate recovery and reduce hospital length of stay with 2 days after liver resection, based on a standardized pre- and post-operative management. In short, the programme consists of preoperative counselling concerning the nature of the preoperative care and the role that the participants play in promoting their own recovery. No premedication will be provided and no preoperative fasting will be instituted while carbohydrate containing liquids will be provided to up to 2 hours prior to surgery. During surgery patients will receive continuous mid-thoracic epidural anaesthesia and short acting anaesthetics, as well as oxygen supplementation. Perioperative fluid overload is avoided and non-opioid pain management is installed. Furthermore, nasogastric tubes are not routinely used and early removal of bladder catheters is standard, as are administration of laxatives and prokinetics, and early and enhanced post-operative feeding and mobilisation.

The ERAS protocol also includes laboratory liver function tests and avoidance of excessive fluids (preoperative and per-operative central venous pressure of < $5 \mathrm{mmHg})^{44,58}$

\section{Follow-up}

Patients are observed during hospital admission. Follow-up visits are planned after two and six weeks, at which time the surveys pertaining to the quality of life will be conducted. All patients, irrespective of the received treatment, will undergo an MRI-scan 12 weeks post-procedure. For patients who underwent selective arterial embolisation, follow-up visits are planned every six months at which time patients also undergo an MRI-scan and annual control of serum AFP levels. For patients who underwent resection of their HCA, follow-up visits are also planned every six months, but in general no MRI-scans will be performed unless for those patients, in whom disease is left in situ, based on the size criterion (size $<5 \mathrm{~cm}$ ).

For the primary endpoint, follow-up is completed 90 days post-operatively. For the secondary endpoint, a 5-year-follow-up after discharge will be viewed as complete. This 5-year-follow-up will grant us the true long-term outcomes following resection and selective arterial embolisation for HCAs and will thereby provide us with a unique cohort within the Netherlands. 


\section{Statistical analysis}

\section{Intention-to-treat}

The analysis will be performed in accordance with the intention to treat principle. However, data of those patients who failed their assigned treatment will be noted, in order to establish the degree of cross-over. Per protocol analysis will be performed but solely for hypothesis generating arguments.

\section{Sample size calculation and statistical analysis}

Since selective arterial embolisation of non-haemorrhaging HCAs is expected to be a less invasive treatment, we hypothesized that the occurrence of post-treatment morbidity and mortality will be reduced. Conversely, patients who undergo selective arterial embolisation as treatment modality will possibly have a longer and more costly follow-up. Moreover, in some patients residual disease may remain in situ, possibly leaving these patients at risk for complications. Reported morbidity and mortality following elective liver resection at MUMC+ are $32 \%$ and $3.4 \%$ respectively. As very limited data is available on using selective arterial embolisation as a treatment for non-ruptured HCAs, assumptions are made based on data from a non-published pilot conducted at MUMC+. Taking all these considerations into account, we therefore assumed that an expected absolute reduction of morbidity and mortality of $25 \%$ percent point and $3 \%$ percent point respectively can be judged as clinically relevant. So, it is expected that intra-arterial emobilisation can reduce the incidence of the primary endpoint from $32 \%$ to $7 \%$. With $80 \%$ power at $5 \%$ alpha (two-sides), sample size calculation requires 46 patients per randomised group in order to determine the difference in treatment effect on the primary endpoint. With loss-to-follow up of 1 patient per arm a total of 94 (2x47) patients will be randomized.

Summary statistics will be obtained using established methods and presented as percentages or median values to assess the quality of the data, comparability of treatment groups and endpoints. Significance levels will be set at $p<0.05$ and all tests will be two-sided.

\section{Data collection and monitoring}

Data are collected via a secured internet module, specifically designed for this trial. Data concerning patient characteristics, post-treatment morbidity, functional recovery, surgical/ anesthesiological parameters, length of stay, quality of life, patient compliance and costs will be prospectively collected using $\mathrm{MACRO}^{\circledR} . \mathrm{MACRO}^{\circledR}$ is a web-based, real-time electronic data management system provided by CTCM (clinical trial centre Maastricht) in which all data will be collected, through e-CRF's (electronic case report forms), validated and stored in compliance with good clinical 
practice (GCP) guidelines. All patient data will be de-identified. Data are collected daily during hospital admission. Preoperatively, and two and six weeks postoperatively, the surveys to assess quality of life (SF-36 and GIQLI) are filled in by the patient. Outpatient department follow-up visits are planned at six months.

Regular contact between the study coordinator and the participating centres will take place.

\section{DISCUSSION}

Although HCAs have a low, albeit rising, incidence, these benign tumours are a substantial risk factor for developing life-threatening bleeding ${ }^{3,5,13-15}$ and also harbour a significant risk of malignant evolution of $4.3 \%{ }^{16,24,59-62}$ Both hazards are thought to be associated with the size of the tumour $(\geq 5 \mathrm{~cm}){ }^{12,18-22,25}$

In general, for lesions smaller than $5 \mathrm{~cm}$ observation is justified, not only based on the smaller risk of complications, but also because of improved radiological reliability which diminishes risks of misclassifying lesions on imaging as being benign. Moreover, repeat imaging does not pose a threat of bleeding nor of tumour cell dissemination and it is non-invasive. However, in case of tumour growth, intervention is advocated. ${ }^{20,21,32}$

Thus far, the recommended treatment for non-bleeding lesions of at least $5 \mathrm{~cm}$ is elective resection of the liver segment containing the HCA. ${ }^{12,16,19,21}$ However, selective arterial embolisation holds a promise in overtaking this position as it has proven to cause significant tumour regression. ${ }^{12,29,31-34}$ The aim of selective arterial embolisation is downsizing of the tumour by complete cessation of the arterial blood flow, which in turn would eliminate the risk of bleeding and would probably concomitantly decrease the hazard of malignant transformation, principally similar to resection of the HCA. ${ }^{12,32,34}$

Furthermore, selective arterial embolisation is thought to be less invasive than hepatic resection, especially within the group of patients for which the incidence of HCA is highest: otherwise healthy, mostly young individuals using oral contraceptives. $^{32,34,63}$

In light of the promising preliminary results of selective arterial embolisation as a treatment for non-haemorrhaging HCAs larger than $5 \mathrm{~cm}$, the risks that accompany resection should play a larger role in determining the optimal treatment strategy. However, only limited experience is available regarding this new indication for selective arterial embolisation as elective treatment for large non-haemorrhaging 
HCAs, since only a few studies ${ }^{32,34}$ on this subject have been published. Therefore, there is need for a large randomised trial comparing both invasive techniques.

\section{Conclusion}

This trial is a randomised controlled multicentre trial set up to compare elective selective arterial embolisation to elective surgical resection for non-haemorrhaging HCAs measuring more than $5 \mathrm{~cm}$ in diameter. Moreover, this trial will provide data on the long-term outcomes in a unique cohort throughout the Netherlands. Results are expected in 2015.

\section{REFERENCES}

1. Grazioli L, Federle MP, Brancatelli G, Ichikawa T, Olivetti L, Blachar A. Hepatic adenomas: imaging and pathologic findings. Radiographics 2001;21(4):877-892; discussion 892-874.

2. Reddy KR, Schiff ER. Approach to a liver mass. Semin Liver Dis 1993;13(4):423-435.

3. Contostavlos DL. Letter: Benign hepatomas and oral contraceptives. Lancet 1973;2(7839):1200.

4. Klatskin G. Hepatic tumors: possible relationship to use of oral contraceptives. Gastroenterology 1977;73(2):386-394.

5. Rooks JB, Ory HW, Ishak KG, Strauss LT, Greenspan JR, Hill AP, et al. Epidemiology of hepatocellular adenoma. The role of oral contraceptive use. Jama 1979;242(7):644-648.

6. Rooks JB, Ory HW, Ishak KG, Strauss LT, Greenspan JR, Tyler CW, Jr. The association between oral contraception and hepatocellular adenoma--a preliminary report. Int J Gynaecol Obstet 1977;15(2):143-144.

7. Alshak NS, Cocjin J, Podesta L, van de Velde R, Makowka L, Rosenthal P, et al. Hepatocellular adenoma in glycogen storage disease type IV. Arch Pathol Lab Med 1994;118(1):88-91.

8. Labrune $\mathrm{P}$, Trioche $\mathrm{P}$, Duvaltier I, Chevalier P, Odievre M. Hepatocellular adenomas in glycogen storage disease type I and III: a series of 43 patients and review of the literature. J Pediatr Gastroenterol Nutr 1997;24(3):276-279.

9. Reddy BS, Rao CV, Rivenson A, Kelloff G. Inhibitory effect of aspirin on azoxymethane-induced colon carcinogenesis in F344 rats. Carcinogenesis 1993;14(8):1493-1497.

10. Socas L, Zumbado M, Perez-Luzardo O, Ramos A, Perez C, Hernandez JR, et al. Hepatocellular adenomas associated with anabolic androgenic steroid abuse in bodybuilders: a report of two cases and a review of the literature. Br J Sports Med 2005;39(5):e27.

11. Soe KL, Soe M, Gluud C. Liver pathology associated with the use of anabolic-androgenic steroids. Liver 1992;12(2):73-79.

12. Leese $\mathrm{T}$, Farges $\mathrm{O}$, Bismuth $\mathrm{H}$. Liver cell adenomas. A 12-year surgical experience from a specialist hepato-biliary unit. Ann Surg 1988;208(5):558-564.

13. Herman P, Pugliese V, Machado MA, Montagnini AL, Salem MZ, Bacchella T, et al. Hepatic adenoma and focal nodular hyperplasia: differential diagnosis and treatment. World J Surg 2000;24(3):372-376.

14. Kerlin P, Davis GL, McGill DB, Weiland LH, Adson MA, Sheedy PF, $2^{\text {nd }}$. Hepatic adenoma and focal nodular hyperplasia: clinical, pathologic, and radiologic features. Gastroenterology 1983;84(5 Pt 1):994-1002.

15. Nagorney DM: Benign hepatic tumors. focal nodular hyperplasia and hepatocellular adenoma. World J Surg 1995;19(1):13-18.

16. Barthelmes L, Tait IS. Liver cell adenoma and liver cell adenomatosis. HPB (Oxford) 2005;7(3):186196. 
17. Charny CK, Jarnagin WR, Schwartz LH, Frommeyer HS, DeMatteo RP, Fong Y, et al. Management of 155 patients with benign liver tumours. Br J Surg 2001;88(6):808-813.

18. Ault GT, Wren SM, Ralls PW, Reynolds TB, Stain SC. Selective management of hepatic adenomas. Am Surg 1996;62(10):825-829.

19. Chiche L, Dao T, Salame E, Galais MP, Bouvard N, Schmutz G, et al. Liver adenomatosis: reappraisal, diagnosis, and surgical management: eight new cases and review of the literature. Ann Surg 2000;231(1):74-81.

20. Terkivatan $\mathrm{T}$, de Wilt JH, de Man RA, van Rijn RR, Zondervan PE, Tilanus HW, et al. Indications and long-term outcome of treatment for benign hepatic tumors: a critical appraisal. Arch Surg 2001;136(9):1033-1038.

21. van der Windt DJ, Kok NF, Hussain SM, Zondervan PE, Alwayn IP, de Man RA, et al. Case-orientated approach to the management of hepatocellular adenoma. Br J Surg 2006;93(12):1495-1502.

22. Vetelainen R, Erdogan D, de Graaf W, ten Kate F, Jansen PL, Gouma DJ, et al. Liver adenomatosis: re-evaluation of aetiology and management. Liver Int 2008;28(4):499-508.

23. Deneve JL, Pawlik TM, Cunningham S, Clary B, Reddy S, Scoggins CR, et al. Liver cell adenoma: a multicentre analysis of risk factors for rupture and malignancy. Ann Surg Oncol 2009;16(3):640648.

24. Stoot JHMB, Coelen RJS, de Jong MC, Dejong CHC. Malignant transformation of hepatocellular adenomas into hepatocellular carcinomas: a systematic review including more than 1600 adenoma-cases. HPB (Oxford) 2010;12(8):509-522.

25. Weimann A, Ringe B, Klempnauer J, Lamesch P, Gratz KF, Prokop M, et al. Benign liver tumors: differential diagnosis and indications for surgery. World J Surg 1997; 21(9):983-990; discussion 990981.

26. Bioulac-Sage P, Balabaud C, Bedossa P, Scoazec JY, Chiche L, Dhillon AP, et al. Pathological diagnosis of liver cell adenoma and focal nodular hyperplasia: Bordeaux update. J Hepatol 2007;46(3):521-527.

27. Thompson MD, Monga SP. WNT/beta-catenin signaling in liver health and disease. Hepatology 2007;45(5):1298-1305.

28. Zucman-Rossi J, Jeannot E, Nhieu JT, Scoazec JY, Guettier C, Rebouissou S, et al. Genotypephenotype correlation in hepatocellular adenoma: new classification and relationship with HCC. Hepatology 2006;43(3):515-524.

29. Erdogan D, Busch OR, van Delden OM, Ten Kate FJ, Gouma DJ, van Gulik TM. Management of spontaneous haemorrhage and rupture of hepatocellular adenomas. A single centre experience. Liver Int 2006;26(4):433-438.

30. Shortell CK, Schwartz SI. Hepatic adenoma and focal nodular hyperplasia. Surg Gynecol Obstet 1991;173(5):426-431.

31. Huurman VA, Stoot JH, van der Linden E, Terpstra OT, Schaapherder AF. Necrosis of a large hepatic tumor after hemorrhage and subsequent selective arterial embolization. World J Gastroenterol 2006;12(37):6059-6061.

32. Stoot JH, van der Linden E, Terpstra OT, Schaapherder AF. Life-saving therapy for haemorrhaging liver adenomas using selective arterial embolization. Br J Surg 2007;94(10):1249-1253.

33. Wheeler PG, Melia W, Dubbins P, Jones B, Nunnerley H, Johnson P, et al. Non-operative arterial embolisation in primary liver tumours. Br Med J 1979;2(6184):242-244.

34. Erdogan D, van Delden OM, Busch OR, Gouma DJ, van Gulik TM. Selective transcatheter arterial embolization for treatment of bleeding complications or reduction of tumor mass of hepatocellular adenomas. Cardiovasc Intervent Radiol 2007;30(6):1252-1258.

35. Belghiti J, Pateron D, Panis Y, Vilgrain V, Flejou JF, Benhamou JP, et al. Resection of presumed benign liver tumours. Br J Surg 1993;80(3):380-383.

36. Liu L, Zhu XD, Wang WQ, Shen Y, Qin Y, Ren ZG, et al. Activation of beta-catenin by hypoxia in hepatocellular carcinoma contributes to enhanced metastatic potential and poor prognosis. Clin Cancer Res 2010;16(10):2740-2750. 
37. Dindo D, Demartines N, Clavien PA. Classification of surgical complications: a new proposal with evaluation in a cohort of 6336 patients and results of a survey. Ann Surg 2004;240(2):205-213.

38. Dorrepaal KL, Aaronson NK, van Dam FS. Pain experience and pain management among hospitalized cancer patients. A clinical study. Cancer 1989;63(3):593-598.

39. McGuire DB. Comprehensive and multidimensional assessment and measurement of pain. J Pain Symptom Manage 1992;7(5):312-319.

40. Paice JA, Cohen FL. Validity of a verbally administered numeric rating scale to measure cancer pain intensity. Cancer Nurs 1997;20(2):88-93.

41. Krebs EE, Carey TS, Weinberger M. Accuracy of the pain numeric rating scale as a screening test in primary care. J Gen Intern Med 2007;22(10):1453-1458.

42. Suurmeijer TP, Doeglas DM, Moum T, Briancon S, Krol B, Sanderman R, et al. The Groningen Activity Restriction Scale for measuring disability: its utility in international comparisons. Am J Public Health 1994;84(8):1270-1273.

43. Maessen J, Dejong CH, Hausel J, Nygren J, Lassen K, Andersen J, et al: A protocol is not enough to implement an enhanced recovery programme for colorectal resection. Br J Surg 2007;94(2):224231.

44. Stoot JHMB, van Dam RM, Busch ORC, R. vH, de Boer M, Olde Damink SWM, Bemelmans MHA, et al. The effect of a multimodal 'fast track'programme on laparoscopic liver surgery - a multicentre pilot study. HPB (Oxford) 2009;11:140-144.

45. Aaronson NK, Muller M, Cohen PD, Essink-Bot ML, Fekkes M, Sanderman R, et al. Translation, validation, and norming of the Dutch language version of the SF-36 Health Survey in community and chronic disease populations. J Clin Epidemiol 1998;51(11):1055-1068.

46. Dubernard G, Rouzier R, David-Montefiore E, Bazot M, Darai E. Use of the SF-36 questionnaire to predict quality-of-life improvement after laparoscopic colorectal resection for endometriosis. Hum Reprod 2008;23(4):846-851.

47. Brazier J, Roberts J, Deverill M. The estimation of a preference-based measure of health from the SF-36. J Health Econ 2002;21(2):271-292.

48. Oostenbrink JB, Koopmanschap MA, Rutten FF. Standardisation of costs: the Dutch Manual for Costing in economic evaluations. Pharmacoeconomics 2002;20(7):443-454.

49. Torgerson DJ, Sibbald B. Understanding controlled trials. What is a patient preference trial? Bmj 1998;316(7128):360.

50. Soyuer I, Ekinci C, Kaya M, Genc Y, Bahar K. Diagnosis of hepatocellular carcinoma by fine needle aspiration cytology. Cellular features. Acta Cytol 2003;47(4):581-589.

51. Wee A, Nilsson B. Highly well differentiated hepatocellular carcinoma and benign hepatocellular lesions. Can they be distinguished on fine needle aspiration biopsy? Acta Cytol 2003;47(1):16-26.

52. Choi BY, Nguyen MH. The diagnosis and management of benign hepatic tumors. J Clin Gastroenterol 2005;39(5):401-412.

53. Chung KY, Mayo-Smith WW, Saini S, Rahmouni A, Golli M, Mathieu D: Hepatocellular adenoma: MR imaging features with pathologic correlation. AJR Am J Roentgenol 1995;165(2):303-308.

54. Laumonier H, Bioulac-Sage P, Laurent C, Zucman-Rossi J, Balabaud C, Trillaud H. Hepatocellular adenomas: magnetic resonance imaging features as a function of molecular pathological classification. Hepatology 2008;48(3):808-818.

55. Lewin M, Handra-Luca A, Arrive L, Wendum D, Paradis V, Bridel E, et al. Liver adenomatosis: classification of $M R$ imaging features and comparison with pathologic findings. Radiology 2006;241(2):433-440.

56. Longerich T, Schirmacher P. [Recent developments in biopsy diagnosis of early and undefined liver tumors]. Z Gastroenterol 2009;47(1):30-36.

57. Rebouissou S, Bioulac-Sage P, Zucman-Rossi J. Molecular pathogenesis of focal nodular hyperplasia and hepatocellular adenoma. J Hepatol 2008;48(1):163-170. 
58. van Dam RM, Hendry PO, Coolsen MM, Bemelmans MH, Lassen K, Revhaug A, et al. Initial experience with a multimodal enhanced recovery programme in patients undergoing liver resection. $\mathrm{Br} J$ Surg 2008;95(8):969-975.

59. Davis M, Portmann B, Searle M, Wright R, Williams R. Histological evidence of carcinoma in a hepatic tumour associated with oral contraceptives. Br Med J 1975;4(5995):496-498.

60. Ferrell LD: Hepatocellular carcinoma arising in a focus of multilobular adenoma. A case report. Am J Surg Pathol 1993;17(5):525-529.

61. Korula J, Yellin A, Kanel G, Campofiori G, Nichols P. Hepatocellular carcinoma coexisting with hepatic adenoma. Incidental discovery after long-term oral contraceptive use. West J Med 1991;155(4):416-418.

62. Pryor AC, Cohen RJ, Goldman RL. Hepatocellular carcinoma in a woman on long-term oral contraceptives. Cancer 1977;40(2):884-888.

63. Stoot JH, van Roosmalen J, Terpstra OT, Schaapherder AF. Life-threatening hemorrhage from adenomas in the liver during pregnancy. Dig Surg 2006;23(3):155. 


\section{Chapter 14}

\section{APPENDIX 1.}

\section{THE ERAS MOBILITY SCALE MMS}

Overview: The Maastricht mobility scale (MMS) is an instrument to compare the level of mobility before and after surgical intervention.

Statements about Activities of Daily Living (ADL) - Can you fully independently:
(1) dress yourself completely?
Yes / No
(2) get in and out of bed?
Yes / No
(3) stand up from sitting in a chair?
Yes / No
(4) wash yourself
Yes / No
(5) get on and off the toilet?
Yes / No
(6) feed yourself?
Yes / No
(7) get around in the house/ward (if necessary with a crane)?
Yes / No
(8) go up and down the stairs?
Yes / No
(9) prepare breakfast or lunch (light meals)?
Yes / No 


\title{
APPENDIX 2. \\ QUALITY OF LIFE QUESTIONAIRE SF-36, VALIDATED DUTCH TRANSLATION FOR DUTCH CENTRES
}

\author{
SF-36 Gezondheidstoestand vragenlijst \\ De volgende vragen gaan over uw gezondheidstoestand
}

INSTRUCTIE: Deze vragenlijst gaat over uw standpunten t.a.v. uw gezondheid. Met behulp van deze gegevens kan worden bijgehouden hoe $u$ zich voelt en hoe goed $u$ in staat bent uw gebruikelijke bezigheden uit te voeren.

Beantwoord elke vraag door het antwoord op de aangegeven wijze te markeren. Als u niet zeker weet hoe $u$ een vraag moet beantwoorden, geef dan het best mogelijke antwoord.

\section{Hoe zou u over het algemeen uw gezondheid noemen?}

(omcirkel één cijfer)

$\begin{array}{ll}\text { Uitstekend } & 1 \\ \text { Zeer goed } & 2 \\ \text { Goed } & 3 \\ \text { Matig } & 4 \\ \text { Slecht } & 5\end{array}$

\section{Hoe beoordeelt u nu uw gezondheid over het algemeen, vergeleken met een jaar geleden?} (omcirkel één cijfer)

Veel beter nu dan een jaar geleden $\quad 1$

Wat beter nu dan een jaar geleden 2

Ongeveer hetzelfde nu als een jaar geleden 3

Wat slechter nu dan een jaar geleden $\quad 4$

Veel slechter nu dan een jaar geleden $\quad 5$

3. De volgende vragen gaan over bezigheden die u misschien doet op een doorsnee dag. Wordt $u$ door uw gezondheid op dit moment beperkt bij deze bezigheden? Zo ja, in welke mate?

\begin{tabular}{lccc} 
& (omcirkel één cijfer op elke regel) \\
\hline BEZIGHEDEN & $\begin{array}{c}\text { Ja, ernstig } \\
\text { beperkt }\end{array}$ & $\begin{array}{c}\text { Ja, een beetje } \\
\text { beperkt }\end{array}$ & $\begin{array}{c}\text { Nee, hele- } \\
\text { maal niet beperkt }\end{array}$ \\
\hline
\end{tabular}

a. Forse inspanning, zoals hardlopen, tillen van zware voorwerpen, een veeleisende sport beoefenen

b. Matige inspanning, zoals een tafel verplaatsen, stofzuigen, zwemmen of fietsen

c. Boodschappen tillen of dragen

d. Een paar trappen oplopen

e. Eén trap oplopen

f. Bukken, knielen of hurken

g. Meer dan een kilometer lopen

h. Een paar honderd meter lopen

i. Ongeveer honderd meter lopen

j. Uzelf wassen of aankleden
1

123

$\begin{array}{lll}1 & 2 & 3 \\ 1 & 2 & 3 \\ 1 & 2 & 3 \\ 1 & 2 & 3 \\ 1 & 2 & 3 \\ 1 & 2 & 3 \\ 1 & 2 & 3 \\ 1 & 2 & 3 \\ 1 & 2 & 3\end{array}$




\section{Chapter 14}

4. Heeft $u$ in de afgelopen 4 weken, een van de volgende problemen bij uw werk of andere dagelijkse bezigheden gehad, ten gevolge van uw lichamelijke gezondheid?

(omcirkel één cijfer op elke regel)

\begin{tabular}{lll}
\hline & JA & NEE \\
\hline a. U besteedde minder tijd aan werk of andere bezigheden & 1 & 2 \\
b. $U$ heeft minder bereikt dan u zou willen & 1 & 2 \\
$\begin{array}{l}\text { c. } U \text { was beperkt in het soort werk of andere bezigheden } \\
\text { d. } U \text { had moeite om uw werk of andere bezigheden uit te voeren (het kostte u bv. extra } \\
\text { inspanning) }\end{array}$ & 2 & 2 \\
\hline
\end{tabular}

5. Heeft $u$ in de afgelopen 4 weken, een van de volgende problemen ondervonden bij uw werk of andere dagelijkse bezigheden ten gevolge van emotionele problemen (zoals depressieve of angstige gevoelens)?

(omcirkel één cijfer op elke regel)

\begin{tabular}{lll}
\hline & JA & NEE \\
\hline a. U besteedde minder tijd aan werk of andere bezigheden & 1 & 2 \\
b. $U$ heeft minder bereikt dan u zou willen & 1 & 2 \\
c. U deed uw werk of andere bezigheden niet zo zorgvuldig als gewoonlijk & 1 & 2 \\
\hline
\end{tabular}

6. In hoeverre hebben uw lichamelijke gezondheid of emotionele problemen u gedurende de afgelopen 4 weken gehinderd in uw normale omgang met familie, vrienden of buren, of bij activiteiten in groepsverband?

$\begin{array}{ll}\text { Helemaal niet } & 1 \\ \text { Enigszins } & 2 \\ \text { Nogal } & 3 \\ \text { Veel } & 4 \\ \text { Heel erg veel } & 5\end{array}$

7. Hoeveel lichamelijke pijn heeft u de afgelopen 4 weken gehad?

(omcirkel één cijfer)

$\begin{array}{ll}\text { Geen } & 1 \\ \text { Heel licht } & 2 \\ \text { Licht } & 3 \\ \text { Nogal } & 4 \\ \text { Ernstig } & 5 \\ \text { Heel ernstig } & 6\end{array}$

8. In welke mate bent u de afgelopen 4 weken door pijn gehinderd in uw normale werk (zowel werk buitenshuis als huishoudelijk werk)?

Helemaal niet

(omcirkel één cijfer)

Een klein beetje

Nogal 3

Veel 4

Heel erg veel 
9. Deze vragen gaan over hoe $u$ zich voelt en hoe het met $u$ ging in de afgelopen $\mathbf{4}$ weken. Wilt u a.u.b. bij elke vraag het antwoord geven dat het best benadert hoe u zich voelde. Hoe vaak gedurende de afgelopen 4 weken.

\begin{tabular}{lllllll} 
& \multicolumn{7}{c}{ (omcirkel één cijfer op elke regel) } \\
& $\begin{array}{l}\text { de hele } \\
\text { tijd }\end{array}$ & meestal & vaak & soms & $\begin{array}{l}\text { heel } \\
\text { soms }\end{array}$ & $\begin{array}{l}\text { geen } \\
\text { enkel } \\
\text { moment }\end{array}$ \\
\hline a. Voelde u zich levenslustig? & 1 & 2 & 3 & 4 & 5 & 6 \\
b. Was u erg zenuwachtig? & 1 & 2 & 3 & 4 & 5 & 6 \\
c. Zat u zo in de put dat niets u kon opvro- & 1 & 2 & 3 & 4 & 5 & 6 \\
lijken? & 1 & 2 & 3 & 4 & 5 & 6 \\
d. Voelde u zich rustig en tevreden? & 1 & 2 & 3 & 4 & 5 & 6 \\
e. Had u veel energie? & 1 & 2 & 3 & 4 & 5 & 6 \\
f. Voelde u zich somber en neerslachtig? & 1 & 2 & 3 & 4 & 5 & 6 \\
g. Voelde u zich uitgeput? & 1 & 2 & 3 & 4 & 5 & 6 \\
h. Was u een gelukkig mens? & 1 & 2 & 3 & 4 & 5 & 6 \\
i. Voelde u zich moe? & & & & & & \\
\hline
\end{tabular}

10. Hoe vaak hebben uw lichamelijke gezondheid of emotionele problemen u gedurende de afgelopen 4 weken gehinderd bij uw sociale activiteiten (zoals vrienden of familie bezoeken, etc)?

Altijd (omcirkel één cijfer)

Soms 3

Zelden 4

Nooit 5

11. Hoe JUIST of ONJUIST is elk van de volgende uitspraken voor u?

\begin{tabular}{llllll} 
& \multicolumn{5}{c}{ (omcirkel één cijfer op elke regel) } \\
\hline & $\begin{array}{l}\text { volkomen } \\
\text { juist }\end{array}$ & $\begin{array}{l}\text { grotendeels } \\
\text { juist }\end{array}$ & $\begin{array}{l}\text { weet } \\
\text { ik niet }\end{array}$ & $\begin{array}{l}\text { grotendeels } \\
\text { onjuist }\end{array}$ & $\begin{array}{l}\text { volkomen } \\
\text { onjuist }\end{array}$ \\
\hline $\begin{array}{l}\text { a. Ik lijk wat gemakkelijker } \\
\text { ziek te worden }\end{array}$ & 1 & 2 & 3 & 4 & 5 \\
$\begin{array}{l}\text { b. Ik ben even gezond als } \\
\text { andere mensen }\end{array}$ & 1 & 2 & 3 & 4 & 5 \\
$\begin{array}{l}\text { c. Ik verwacht dat mijn } \\
\text { gezondheid achteruit zal } \\
\text { gaan }\end{array}$ & 1 & 2 & 3 & 4 & 5 \\
$\begin{array}{l}\text { d. Mijn gezondheid is uit- } \\
\text { stekend }\end{array}$ & 1 & 2 & 3 & 4 & 5 \\
\hline
\end{tabular}




\section{APPENDIX 3.}

\section{DAILY GUIDELINE OF THE POSTOPERATIVE CARE OF PATIENTS}

UNDERGOING A LIVER RESECTION IN THE ERAS PROGRAMME.

Day before surgery:

- Normal oral nutrition until midnight

- No pre-anaesthetic medication

- Laboratory tests

Day of surgery

- Carbohydrate drinks up to 2 hrs prior to surgery

- Midthoracic epidural analgesia (low local anaesthetic+ low dose opioïd)

- Short-acting i.v. anaesthetic agent

- Preferably no nasogastric drainage, but when used remove after surgery

- Use warm i.v. fluids and upper and lower body air-warming device

- Avoid excessive i.v. fluids (pre- and per-operative CVP $<5 \mathrm{mmHg}$ )

- No routine drainage of the peritoneal cavity

- Patient sent to recovery ward

- Restart oral intake of water / nutrition

Post Operative Day (POD) 1

- Patient sent to surgical ward

- Patient mobilizes a minimum of four times a day

- Discontinuation of i.v.-fluids

- Patient drinks at least 1.5 litre

- Normal diet

- Continue portable epidural analgesia (local anaesthetic-low dose opioïd)

- Remove urinary catheter

- 1000 mg Paracetamol 6 hourly

- 1000 mg Magnesiumoxide twice daily

- Laboratory tests

POD 2

- Continue portable epidural analgesia with local anaesthetic-

- Stop low dose opioïds

- Continue mobilization a minimum of four times per day

- 1000 mg Paracetamol 6 hourly

- Normal diet

POD 3

- Stop epidural analgesia

- Start NSAIDs

- Continue mobilization

- Normal diet

- Laboratory tests

- Check discharge criteria

- Outpatient appointment made on postoperative day 10,11 or 12

POD 4

- Check discharge criteria

- Patient receives cell phone number HPB-surgeon on duty

- Discharge 


\section{Chapter 15}

\section{Open versus laparoscopic left lateral hepatic}

sectionectomy within an Enhanced Recovery After Surgery (ERAS ${ }^{\circledR}$ ) programme (ORANGE II Trial): Study protocol for a randomised controlled trial

R.M. van Dam, E.M. Wong-Lun-Hing, G.J.P. van Breukelen, J.H.M.B. Stoot, J.R. van der Vorst, M.H.A. Bemelmans, S.W.M. Olde Damink, K.Lassen, C.H.C. Dejong

ORANGE II Study Group

Adapted from Trials.2012,6;13:54

Presented to the European-African Hepato-Pancreato-Biliary Association in Cape Town, South Africa, April 2011. 


\section{ABSTRACT}

Background: The use of laparoscopic liver resection in terms of time to functional recovery, length of hospital stay (LOS), long-term abdominal wall hernias, costs and quality of life (QOL) has never been studied in a randomised controlled trial. Therefore, this is the subject of the international multicentre randomised controlled ORANGE II trial.

Methods: Patients eligible for left lateral sectionectomy (LLS) of the liver will be recruited and randomised at the outpatient clinic. All randomised patients will undergo surgery in the setting of an ERAS programme. The experimental design produces two randomised arms (open and laparoscopic LLS) and a prospective registry. The prospective registry will be based on patients that cannot be randomised because of the explicit treatment preference of the patient or surgeon, or because of ineligibility (not meeting the in- and exclusion criteria) for randomisation in this trial. Therefore, all non-randomised patients undergoing LLS will be approached to participate in the prospective registry, thereby allowing acquisition of an uninterrupted prospective series of patients. The primary endpoint of the ORANGE II trial is time to functional recovery. Secondary endpoints are postoperative LOS, percentage readmission, (liver-specific) morbidity, QOL, body image and cosmetic result, hospital and societal costs over 1 year, and long-term incidence of incisional hernias. It will be assumed that in patients undergoing laparoscopic LLS, length of hospital stay can be reduced by two days. A sample size of 55 patients in each randomisation arm has been calculated to detect a 2 day reduction in LOS (90\% power and $\alpha=0.05$ (two-tailed)).

The ORANGE II trial is a multicentre randomised controlled trial that will provide evidence on the merits of laparoscopic surgery in patients undergoing LLS within an enhanced recovery ERAS programme.

Trial registration: ClinicalTrials.gov NCT00874224. 


\section{BACKGROUND}

Liver resection for colorectal metastases is the only potentially curative therapy, and has become the standard of care in appropriately staged patients, offering 5year survival rates of approximately $35-40 \% .{ }^{1}$ For symptomatic benign lesions and those of uncertain nature or large size, liver resection is also a widely accepted treatment. Within the framework of optimising post-operative recovery and/or producing a shorter length of stay (LOS) in hospital, laparoscopic surgery and enhanced recovery programmes have recently been introduced for liver surgery.

Laparoscopic liver resection was first described in $1995 .^{2}$ Over the past decade the method has gained wide acceptance for various liver resection procedures. ${ }^{3-9}$ Multiple retrospective case series and reviews comparing open with laparoscopic liver resection indicate that laparoscopic liver resection can be used safely for both malignant and benign liver lesions. ${ }^{10-15}$ Recent publications from expert centres show that a substantial part of the total volume of major and minor liver resections is performed laparoscopically, and results are good. ${ }^{16,17}$ Laparoscopic liver resection is associated with shorter LOS, less postoperative pain, earlier recovery, and better quality of life (QOL). ${ }^{9,13,18,19}$ Comparing patients undergoing an open left lateral sectionectomy (LLS) of the liver with those undergoing laparoscopic LLS, both Vigano et al. and Carswell et al..$^{20,21}$ found no significant difference in operating time between the two groups. In addition, the median length of postoperative LOS was significantly less ( 6 vs. 9 days, $P<0.01$ ) after laparoscopic resection. ${ }^{3}$ Furthermore, no evidence of a compromised oncologic clearance in laparoscopic liver resection has been found. ${ }^{3,13}$ However, recovery and LOS are not only dependent on the type of surgery or procedure, and other variables should also be taken into account.

The Enhanced Recovery After Surgery (ERAS) programme has been introduced to improve postoperative care. This multimodal programme, derived from Kehlet's pioneer work in the 1990s for multimodal surgical care, involves optimisation of several aspects of the perioperative management of patients undergoing major abdominal surgery. In patients undergoing segmental colectomy, the ERAS programme enabled earlier recovery and consequently shorter $\operatorname{LOS}^{22-25}$ Furthermore, a reduction of post-operative morbidity in patients undergoing intestinal resection was reported. ${ }^{26-29}$ These results stimulated liver surgeons of the ERAS ${ }^{\circledast}$ group (Maastricht, Edinburgh and Tromsö) to adapt the ERAS programme to patients undergoing open liver resection. Van Dam et $a{ }^{30}{ }^{30}$ found a significantly reduced LOS after open liver resection when patients were managed within a multimodal ERAS programme. Besides a reduction in median total LOS from 8 to 6 days (25\%), the data also suggested that a further reduction in stay could be possible as there was a delay between the recovery and actual discharge of the patients [30]. Moreover, Stoot et al. found retrospectively that there was a further reduction in LOS from 7 days to 5 days when patients were operated laparoscopically and managed within 
an ERAS programme. ${ }^{31}$ In that study there was also a delay between recovery and actual discharge of the patients. Previously, Maessen et al. reported a median delay to discharge of 2 days after patients had functionally recovered after colonic surgery managed within an ERAS programme. ${ }^{32}$ This delay is often linked to patient age, hospital logistics, and absence of social and/or homecare support.

In most reported trials aiming at earlier recovery or a reduction in LOS, type of surgery and/or perioperative management were not standardised. In addition, the added value of laparoscopic LLS compared with open left lateral sectionectomy within an ERAS programme in terms of time to functional recovery, LOS in hospital, costs, and QOL has never been studied in a randomised controlled trial (RCT). However, randomisation of patients undergoing open or laparoscopic liver resection is hazardous. It is to be expected that experienced centres will be reluctant to randomise patients because of the absence of clinical and patient equipoise for laparoscopic resection. To capitalise on both centres with and without preference for laparoscopic liver surgery, and to thereby acquire an uninterrupted prospective series of patients, an alternative trial design with two randomisation arms (open versus laparoscopic surgery) and a prospective registry has been constructed for the ORANGE II trial. The combination of an RCT and a prospective registry will improve overall power and strengthen the external validity and generalisability of study results. $^{33-35}$

\section{METHODS}

\section{Ethics approval}

The study has been approved by the Medical Ethical Review Board of the Maastricht University Medical Centre, Maastricht, The Netherlands trial number NL 25591.068.08 / MEC 08-2-110. Ethics consent will also be obtained from the national or regional ethics boards in each participating country. Patients willing to participate in this trial will receive both verbal and written information at the time of recruitment in the outpatient clinic. In accordance with the local medical ethics committee all participating sites will provide an independent surgeon or physician if needed. An independent surgeon (M. Poeze) has been appointed for the Maastricht University Medical Centre to answer questions. Confidentiality is guaranteed by assigning the participators an encoded trial number. This indicates that only the physician with the decoding 'key' will know which code number has been assigned to any patient. All trial data will be saved during the trial and stored on a server, and patients will be asked to consent to future analysis of these data. Withdrawal from the trial at any time or for any reason will not hold any form of consequences for the patient, and data from these patients will be deleted. 


\section{Study design}

The ORANGE II trial is a prospective superiority study with an experimental design, using two double-blinded randomised controlled arms and a prospective registry to determine whether laparoscopic surgery is to be preferred over open surgery in patients undergoing a LLS and participating in an enhanced recovery programme. In the participating randomising centres, patients, nurses and the ward physician (but not the operating surgeon) will be blinded for the type of intervention up to and including postoperative day (POD) 3. They will record the functional recovery criteria twice daily. Only the investigator and operating surgeons will know the actual procedure. The blinded ward physician(s) will decide on whether a patient will be discharged or not.

However, randomisation of patients undergoing open or laparoscopic liver resection is hazardous as previously explained. Moreover, another potential source of bias exists when randomising patients with a strong treatment preference. When patients cannot be blinded to their treatment allocation (POD 3) they may be resentful and demoralised if they do not receive their preferred treatment, and consequently they may have poor compliance. By contrast, patients receiving their preferred treatment may have above-average compliance.

Thus to capitalise on centres both with and without preference for laparoscopic liver surgery, and thereby to acquire an uninterrupted prospective series of patients, all non-randomised patients undergoing a LLS will be approached to participate in the prospective registry. Registration of these patients is imperative to guarantee a consecutive series of patients and also because the absence of such a series may restrict generalisation of the results, as randomised participants may not in fact be representative. ${ }^{36}$ The combination of an RCT and a prospective registry will improve overall power and strengthen the external validity and generalisability of study results. ${ }^{33-35}$ This non-randomised registry group will be analysed for centre and centre by treatment interaction as an observational study. Medical centres that wish to participate in this trial, but with liver surgeons early in the laparoscopic learning curve, will be accompanied during the procedure by an experienced proctoring laparoscopic HPB-surgeon.

\section{Primary and secondary endpoints}

The primary endpoint of the ORANGE II trial is time to functional recovery. A patient is fully functionally recovered when all of the following five criteria are satisfied: 1) adequate pain control with oral analgesia; 2) restoration of mobility to an independent level; 3) absence of intravenous fluid administration; 4) ability to eat solid foods; and normal or decreasing serum bilirubin level and international normalised ratio. 
It is medically justified to discharge patients when the criteria for full functional recovery are met and if the patient is willing to go home. Secondary endpoints include postoperative LOS in hospital, percentage of readmissions, total morbidity (both general and procedure related), composite endpoint of liver-surgery-specific morbidity, QOL, body image and cosmesis, reasons for delay of discharge after functional recovery, hospital and societal costs over 1 year, and long-term incidence of incisional hernias.

\section{Morbidity}

The preoperative morbidity status of patients will be measured using the American Society of Anesthesiologists (ASA) scale. The Portsmouth modification of the Physiological and Operative Severity Score for the Enumeration of Mortality and Morbidity (P-POSSUM) will be used to evaluate the risk of perioperative morbidity and mortality. Post-operative morbidity is rationally predictable, with hemorrhagic complications occurring predominantly during surgery or in the early postoperative phase, and biliary complications, intra-abdominal abscess, or liver failure in the later postoperative phase. Wound infection and sepsis will be additional complications that require monitoring. Morbidity will be classified and analysed according to the validated classification for postoperative morbidity as described by Dindo et al. $^{37}$

\section{Liver resection-specific composite endpoint}

In this trial, we will also use a well-defined liver surgery-specific composite endpoint, as suggested by van den Broek et al. ${ }^{38}$ This endpoint is a parameter composed of a combination of procedure-specific complications, which is considered as a single, dichotomous outcome: operative mortality, intra-abdominal haemorrhage, ascites, bile leakage, intra-abdominal abscess, and post-resectional liver failure. These components, which are all specific to liver surgery and have substantial clinical relevance, reflect complications rated as Dindo grade 3-5. A composite score of 1 (failure) will reflect the occurrence of at least one of the above liverspecific complications, and a score of 0 (success) will be assigned if none of these occur.

\section{Quality of life}

To assess QOL in patients undergoing laparoscopic versus open LLS, the Dutch version of the EuroQol five-dimension (EQ-5D) status test in Dutch centres and the translated EQ-5D for international centres will be used. The EQ-5D is a standardised instrument for use as a measure of health outcome, which consists of the five di- 
mensions of mobility, self-care, usual activities, pain/discomfort, and anxiety/depression, with three levels each and a rating on the EQ visual analogue scale (VAS; 0-100). ${ }^{39-41}$ Furthermore, the European Organisation for Research and Treatment (EORTC) 30-item post-cancer QOL questionnaire (QLQ-C30; with the liver metastases (LM21) module will be used for liver-specific treatment measurements. ${ }^{42}$ Assessment of the patients' QOL will be performed at the time of consent, discharge and 10 days, 3, 6 and 12 months after discharge.

\section{Body image and cosmesis}

To evaluate differences in postoperative body image and cosmesis, the Body image Questionnaire (BIQ) will be used, ${ }^{43,44}$ which consists of eight questions about body image and cosmesis. The body image assessment will be performed preoperatively at time of consent. Both the body image and cosmesis assessment will take place at discharge, and at 10 days, 3 months, 6 months and 12 months after discharge.

\section{Hospital and societal costs}

The economic evaluation will include a cost-utility analysis from a Dutch societal perspective. The incremental costs per quality adjusted life year (QALY) gained will be based on utility scores from the EQ-5D. ${ }^{39-41}$ All hospital expenses (direct and indirect) related to both interventions will be monitored. In addition, a cost questionnaire offered at the regular follow-up consultation (3, 6 and 12 months) will help assess the societal and individual costs outside health care relating to patients' absence, impaired mobility, work, or normal daily activities. Unit prices will be based either on prices from the participating hospital financial departments or will be extrapolated using Dutch guidelines for cost calculation. ${ }^{45}$

\section{Incidence of incisional hernias}

Incisional hernia after open surgery is a well-known complication of surgery, with an incidence of up to $20 \%$ after a 10 -year period. ${ }^{46}$ In patients undergoing a sigmoid resection, Anderson et al. found that laparoscopic resection led to a significantly lower incidence of incisional hernia compared with open surgery. ${ }^{47}$ Furthermore, in two retrospectively analysed series of patients who received a partial hepatectomy, different types of incisions were compared. D'Angelica et al. reported that the common incisions used for partial hepatectomy were the Mercedes incision and extended right subcostal (ERSC) incision, and that the ERSC incision provides adequate, safe access and is associated with fewer long-term wound complications (9.8\% vs $4.8 \%, P<0.001) .{ }^{48}$ More recently, Togo et al. reported frequencies of incisional hernia after median, J-shaped, right transverse incision with a vertical exten- 
sion at the midline from the subumbilical region to the xiphoid process (RTVE), and reversed $\mathrm{T}$ incisions to be $6.3 \%, 4.7 \%, 5.4 \%$, and $21.7 \%$, respectively. A diagnosis of 'no hernia' required a minimum follow-up of 12 months. ${ }^{49}$

To assess the incidence of incisional hernias in patients undergoing laparoscopic and open LLS, they will be contacted at a mean time of 1 year after resection to undergo ultrasonography to assess the incidence of incisional hernia.

\section{Study population}

Every patient requiring a LLS will be identified and informed at the outpatient clinic about open and laparoscopic liver resection. Only patients meeting the inclusion and exclusion criteria will be approached for randomisation. After reading the ORANGE II trial patient information and being allowed 1 week for consideration, patients will be asked for their informed consent. All patients ineligible for randomisation will be approached for participation in the prospective registry. If patients express an explicit preference, they will be allocated to the prospective registry and interviewed to ascertain the reasons for their preferences. Personal written informed consent will be obtained for all groups. Randomisation will be carried out through the ORANGE II trial website using web-based randomisation software (TENALEA ${ }^{\circledR}$; www.tenalea.com).

Patients will be approached for randomised inclusion if they meet each of the following inclusion criteria: require LLS; willingness to participate in the study; able to understand the nature of the study and what will be required of them; are men or non-pregnant, non-lactating women between the ages of 18 and 80 years of age; have a body mass index of between 18 and 35; and have ASA grading of I to III.

The exclusion criteria are: liver resection other than LLS; underlying liver disease; unwillingness to participate; inability to give written informed consent; and ASA grading of IV to $\mathrm{V}$. 


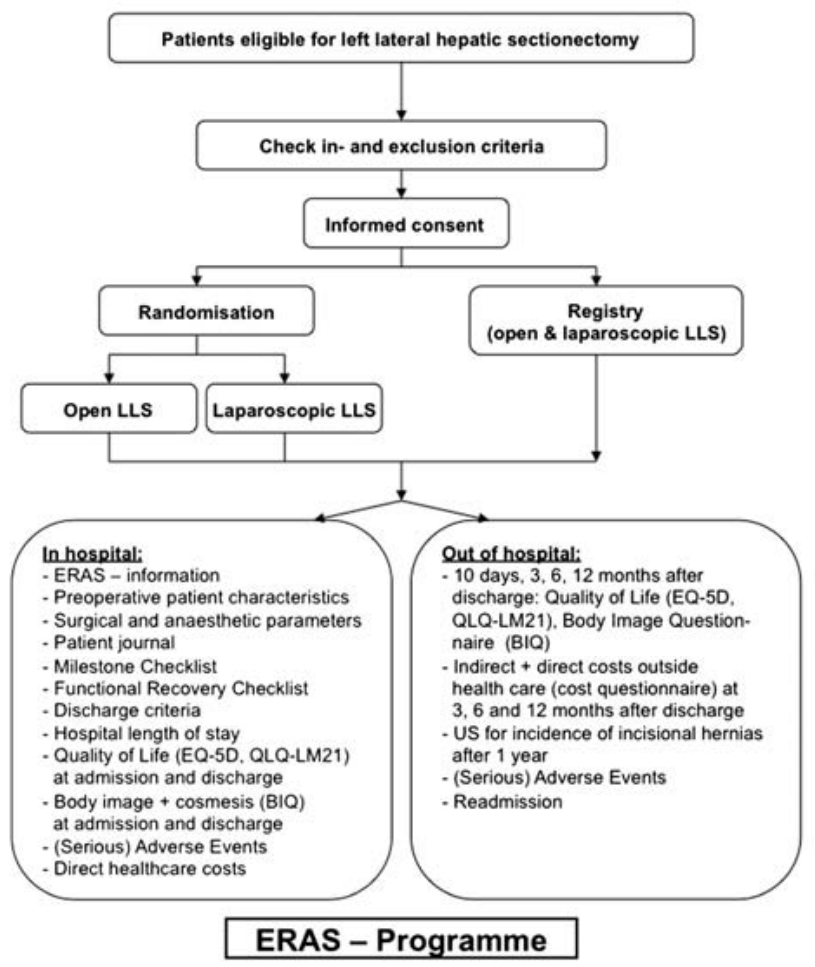

Figure 1 Orange II trial flowchart.

\section{ERAS programme}

All patients will participate in the ERAS liver programme, with a standardised perioperative management. Daily guideline of post-operative care of patients undergoing a hepatectomy in the Enhanced Recovery After Surgery (ERAS) programme (Figure 2).

\section{Functional recovery criteria}

The evaluation of time to functional recovery will start on POD 0 and will be scored twice daily until discharge from the hospital. The discharge process starts at the pre-admission counseling session, during which any special needs of the patients will be determined (for example, homecare or social support, transport. Before admittance, any problem that could delay discharge will be identified and addressed. Patients will only be discharged when they have met the functional recovery criteria and are willing to go home. Reasons to delay discharge after functional 
recovery will be monitored and documented. Functional recovery criteria and LOS in hospital will be independently monitored and analysed.

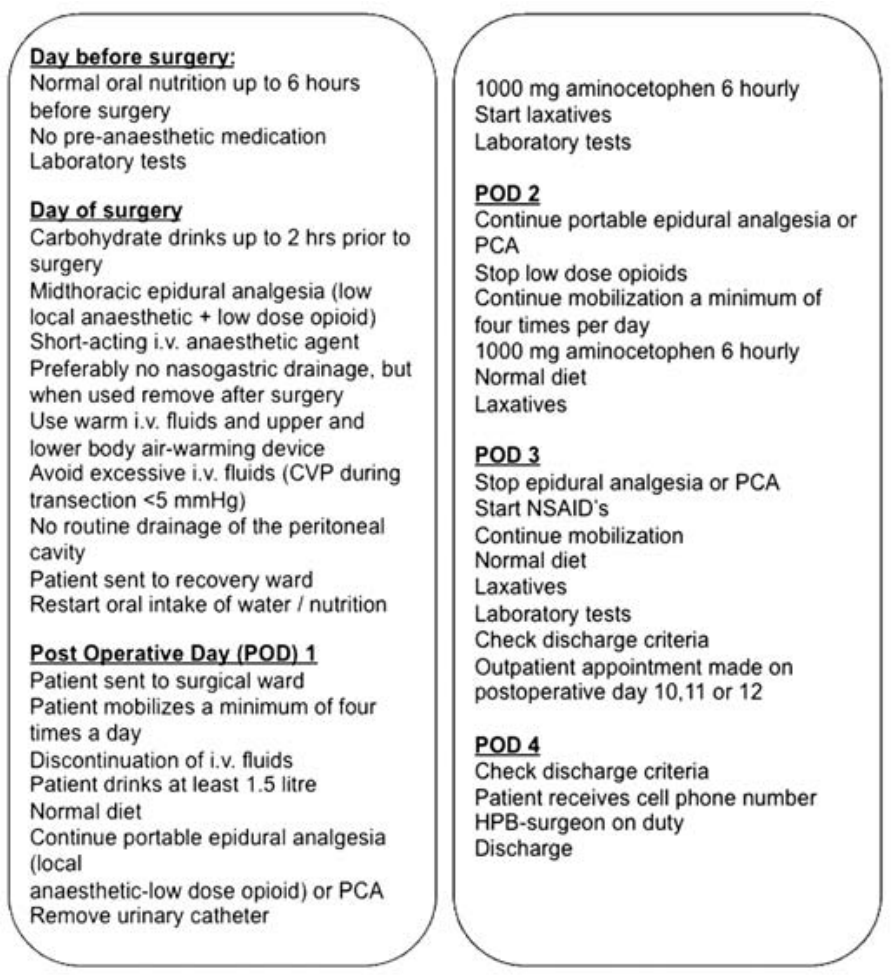

Figure 2 Daily guide of postoperative care of patients undergoing a hepatectomy in the ERAS programme.

\section{Adequate pain control with oral analgesics}

Postoperative pain will be systematically registered twice daily using the validated verbally administered 11-point numeric rating scale (NRS-11, 0 to 10). ${ }^{50-53}$ Members of a specialised pain team will ask patients to rate the intensity of their current pain on a scale of 0 (no pain) to 10 (worst possible pain), with pain rated as 'mild' (1 to 3) 'moderate' (4 to 6) or 'severe') 7 to $10 . .^{54}$ The NRS-11 seems to be better accepted by most patients and to be at least as sensitive and valid as the more traditional VAS ratings. ${ }^{53}$

\section{Tolerance of solid food}

Fluid and solid food intake will be monitored and must return to normal, that is, when oral intake of water or normal food is resumed and continued for at least 24 
hours. Furthermore, the incidence of postoperative nausea and vomiting, which obviously influences intake, will be monitored postoperatively until day 6 using a scale ranging from 0 (no nausea) to 10 (worst possible nausea), and where necessary, be countered prophylactically by antiemetic treatment,

\section{Mobility}

To assess the difference between the preoperative and postoperative mobility level, the ERAS Mobility Scale (EMS) has been developed from the Groningen Activity Restriction Scale. ${ }^{55}$ The EMS assesses 10 basic actions to compare the level of mobility before and after surgical intervention. When the patient is able to perform 8 of the 10 items, they are independently mobile. Patients will be assessed whether they are able or not to independently perform these basic actions fully. Daily the assessment will be repeated and compared with the preoperative baseline score until mobility at an independent or preoperative level is achieved.

\section{Statistical analysis}

\section{Sample size}

Because laparoscopic liver surgery focuses on accelerated recovery, time to functional recovery is used as the primary outcome parameter. Owing to the lack of hard evidence about the reduction in time to functional recovery after liver surgery, we have chosen to use the parameter that most accurately approaches our primary endpoint for our power calculation (LOS). Based on a retrospective analysis of 31 patients in both ERAS and non-ERAS settings, who have undergone LLS from 1990 to the present time, the mean \pm SD post-operative hospital stay for a LLS in the Maastricht University Medical Centre is $6 \pm 2.73$. It therefore seems that that in patients undergoing laparoscopic LLS, time to functional recovery is reduced in comparison to patients undergoing the open procedure. We are aiming for a reduction in time to functional recovery of 2 days. A sample size of $2 \times 40$ patients in the randomisation arms will be sufficient to show a 2-day reduction with a power of $90 \%$ and a level of significance at $\alpha=0.05$ (two-tailed, given a within-arm SD of 2.73 with effect size $d=0.73$ ). Assuming an expected withdrawal rate of $\leq 10 \%$ during the trial, the participation of at least 10 centres, and the required addition of one randomised patient per arm for every additional participating centre (C) to compensate for the loss of degrees of freedom incurred in the data analysis, which takes centre and treatment $\times$ centre effects into account, a total sample size of 110 $(n=2 \times 55)$ will be required. 
For all secondary outcome measures, the power will be $75 \%$ after correction for multiple testing with two-tailed $\alpha=0.01$, assuming the same effect size $(d=0.73)$ as for the primary outcome. An interim analysis of the primary outcome, using Snapinn's method, will be performed after inclusion of $50 \%$ of the sample to avoid unnecessary inclusion of too many patients in this ORANGE II trial. ${ }^{56}$

\section{Descriptive statistics}

The primary outcome parameter of time to functional recovery and the secondary parameter of LOS in hospital will be given in days, with a median and range. Morbidity will be classified according to the classification described by Dindo et al. and defined as a dichotomous composite endpoint, while readmission will be given as a percentage. Scores for quality of life, body image and cosmesis will be given as mean and standard deviation per time point per treatment arm. Hospital costs will be given as median and range. Long-term incidence of incisional hernia will be reported and analysed.

\section{Univariate analysis}

The primary outcome measure of time to functional recovery will be measured in days, and will be analysed with fixed-effect regression that will take centre and treatment $\times$ centre interaction into account as fixed effects. If the actual number of centres and the sample size per centre allow random effects analysis, this will also be performed and this analysis will have the same power as the planned fixed effects analysis if the design effect does not exceed 1.2. With a sample size of 10 patients per centre, the design effect is 1.2 if the intraclass correlation (ICC) is 0.02 , where the ICC is based on treatment $\times$ centre interaction. ${ }^{57}$

All secondary outcomes as measured at discharge will be analysed by fixedeffect regression using linear regression for quantitative outcomes and logistic regression for binary outcomes, and including the baseline measure as a covariate to improve power and precision. In addition to $P$-values, confidence intervals for all effects will be reported. Morbidity will be classified as described by Dindo et al., but will be presented as raw data only because the required sample size for intervention effects on morbidity is much larger than the calculated sample size for this trial. $^{58}$

\section{Economic evaluation}

The economic evaluation will include a cost-utility analysis from a societal perspective. The time horizon of this evaluation will be the same as the duration of the trial, that is, 12 months. All costs (direct and indirect) related to both interventions 
will be calculated. The final cost calculation of unit costs will be based on a combined bottom-up and top-down approach. In accordance with Dutch guidelines for cost calculation, indirect healthcare costs will not be taken into account. In addition, resource use will be measured by use of primary data that is registered in our case record forms (CRFs) by use simple checklists. Furthermore, a questionnaire will be used to survey the direct non-healthcare costs related to travelling, impaired mobility and domiciliary care (for normal daily activities). The incremental, indirect non-healthcare costs per QALY gained will be based on the utility scores from the EQ-5D. ${ }^{39-41}$ For all direct healthcare costs, the unit prices will be based either on prices from the hospital financial department or the Dutch guidelines for cost calculation. $^{45}$

\section{Registry}

The prospective registry of patients who cannot be randomised because of ineligibility or because of explicit treatment preference on the part of the patient or surgeon will be analyzed as an observational study. In addition, data from the registry will be analyzed for interaction between treatment, centre, and study type (randomised or not). On condition that there is no interaction between treatment, centre, and study type, and that the observational study does not suffer from severe confounding (because adjusting for that strongly reduces the power of the observational study), pooling of both studies should give more power than separate analyses of either study. Possible confounders will be registered in the CRFs. The inclusion of the prospective registry in the trial design will create an uninterrupted case series, which will increase external validity and generalisability.

\section{Data collection}

Data concerning patient characteristics, functional recovery, surgical and anaesthesiologic parameters, morbidity, LOS, QOL, patient compliance, and costs will be prospectively collected using both paper CRFs and an open source clinical trial software platform (OpenClinica ${ }^{\circledR}$; Ikaza Research, Cambridge, MA, USA) that uses eCRFs for electronic data capture and clinical data management, which are validated and stored in compliance with good clinical practice guidelines. The e-CRFs will be stored in a secured database (Oracle Cor., Redwood Shores, CA, USA), and as stated previously, all patient data will be encoded to ensure privacy.

\section{Monitoring}

For this trial, a Data and Safety Monitoring Board (DSMB) has been appointed that will consist of three members: a chairperson, an independent statistician, and a 
medical specialist. In a concerted effort a DSMB charter will be developed, and all three members will sign a non-competing interest form. The DSMB will be responsible for safeguarding the interests of trial participants, assessing the safety and efficacy of the interventions during the trial, and monitoring the overall conduct of the clinical trial.

\section{Intention to treat}

Analysis of all patients will be performed according to the intention-to-treat principle: patients will be analysed as randomised or as planned in the non-randomised prospective registry, and all patients will be included in the data analysis with proper methods for handling missing data.

\section{DISCUSSION}

Several authors have indicated that laparoscopic liver resection has many benefits over conventional open liver resection. However, this has never been proven in an RCT, and what the primary endpoint should be for an RCT comparing open and laparoscopic liver resection is a subject to debate. Using either liver surgery-related mortality or liver surgery-specific morbidity as an endpoint is not feasible, because patient accrual would take many years and be a logistically major global effort. ${ }^{58}$ LOS in hospital, time to recovery, long-term incisional hernias, body image, and costs are potential candidates because improvements in these are some of the possible benefits. Laparoscopic liver resection is appealing for many surgeons and patients, but the learning curve for the surgeon is thought to be long and costly for hospital budgets. However, operating times in laparoscopic LLS tend to be shorter, and may compensate for expenses in technology and consumables. ${ }^{31,59}$ Moreover, the existing trials in liver surgery have not evaluated time to recovery or LOS in hospital after laparoscopic liver resection within an enhanced recovery programme. The more rapid recovery reported after enhanced recovery programmes may be further accelerated as a consequence of small incisions in laparoscopic surgery. In addition, learning curves for laparoscopic left lateral resection or anterior segments seem to be reasonably short for liver surgeons with advanced laparoscopic experience. ${ }^{60}$ The question remains whether an RCT is necessary to prove that laparoscopy should be accepted as the preferred a method to perform liver resection. In the Louisville consensus meeting on laparoscopic liver surgery, it was stated that laparoscopic LLS should be standard practice in experienced hands. ${ }^{61}$ However, this may have been a subjective vision of a subset of opinion leaders, because long experience with both open and laparoscopic liver surgery was the main characteristic of those attending the meeting. Undoubtedly, the dissemina- 
tion phase of laparoscopic liver surgery has started, and it is to be expected that many surgeons will adopt this technique in the future. A multinational multicentre prospective registry, a well-organised multicentre RCT, training programmes, and quality control measures are of great importance during this adoption period. ${ }^{33}$

It is well recognised that a well-conducted double-blind RCT provides the highest level of evidence to prove the possible benefits of laparoscopic liver resection. However, performing an RCT in surgery is not without difficulties, and alternative trial designs may be necessary. ${ }^{33,34,62}$ First, the intervention needs to be tested in a standardised environment, and the properties of the intervention should remain unchanged during the trial period. This seems impossible for an intervention such as laparoscopic liver surgery in a multicentre RCT. Experience varies between participating centres, and will vary over time. Moreover, local standards for perioperative care are different. Both LLS and the ERAS ${ }^{\circledR}$ enhanced recovery protocol provide the standardisation needed. The learning curve of a LLS is short in centres with experience in liver surgery and advanced laparoscopy. The use of proctor surgeons in centres with limited experience in laparoscopic liver surgery the operative techniques can be reasonably standardised, and this should eliminate learning curve influences on outcome parameters. Quality of the surgery can be assured by digital video recording.

Second, the intervention should be double-blinded. Although double blinding in a surgical trial is difficult, using a fixed abdominal dressing for 3 days after surgery is feasible, and should prevent both ward caregivers and patients from knowing the type of intervention.

Third, it is reasonable to query whether this is now the right time to perform an RCT and whether the results of the trial will be valid for the more general surgical community. A recent review of the results of laparoscopic liver resection in 2,804 patients showed that laparoscopic liver resection in expert centres is feasible and safe for both minor and major liver resections. ${ }^{36}$ The percentages of liver resections performed laparoscopically now ranging from $25 \%$ to $65 \%$ in high-volume expert centres such as University Hospital Southampton NHS (Southampton, UK), Henri Mondor (Paris, France), UPMC (Pittsburgh, USA), UZ Leuven (Leuven, Belgium) and Rikshospitalet (Oslo, Norway). ${ }^{36,63}$ Although it is to be expected that many centres worldwide will adopt laparoscopic liver resection as a more or less standard procedure in the near future, there are still many patients and surgeons that prefer the open procedure long beyond the learning curve. In parallel with the development of laparoscopic liver surgery, 'fast-track' programmes in various areas of surgery, including liver surgery, are gaining popularity. Therefore, this seems to be the right time for this RCT to be performed. The multicentre character of the ORANGE II trial with randomisation of patients and surgeons with treatment equipoise and a prospective registry to cover both surgeons who believe that based on their laparoscopic experience randomisation is not ethically justified and patients with a strong 
treatment preference will provide external validity. This trial design capitalises on rather than ignores the differences between patients, will provide more robust outcome data, and should lead to continuous performance monitoring after the trial. $^{35,62}$

The key question clearly is as to whether this RCT is really necessary. The benefits of laparoscopic liver resection are not beyond reasonable doubt, and although data are increasingly becoming available, recent publications do not provide sound data on time to recovery. Worldwide, median LOS in hospital for open and laparoscopic resections varies from 4 to 8 days. ${ }^{17,30,64-66}$ Reasons for delay in discharge and discharge location are often absent, and a clear definition of recovery has not been used to date in any of the publications. Departing from the standpoint that an RCT should be conducted, the question is which sample size should be used? In our opinion, a reduction of only 1 day in time to recovery or LOS in hospital after laparoscopic resection would be a disappointingly low gain. To prove such a reduction, 320 patients would be needed ( $\alpha=0.05$ and power of $90 \%$ ), making the trial unlikely to be accomplished. Based on available reports, a 2-day reduction should be possible, ${ }^{17,31,66}$ and reduces the sample size to 110 patients undergoing LLS. This number is reasonably moderate, and it is to be expected that patient accrual will be accomplished within 1-2 years.

It should be realised that many centres have introduced laparoscopic liver surgery programmes in the absence of a central reporting or certifying agency. In our opinion, laparoscopic LLS should function as a model for further dissemination of laparoscopic techniques in hepatic surgery. The left lateral segment of the liver has been a natural first step for a laparoscopic resection given the peripheral anatomical location (thin liver segment, minimal requirement for biliary dissection, and ease of controlling the left portal pedicles and left hepatic vein), and has been proven to be safe and feasible with reproducible results. ${ }^{20,36}$ The implementation of the laparoscopic LLS may not only serve as a guide to develop and master programmes for major laparoscopic hepatic resections, but may also be used as an introduction for centres new to laparoscopic approaches in liver surgery. To adopt laparoscopic liver resection safely, certification for centres, surgeons, and units should be available through the International Hepatobiliary (HPB) Association, and national and international HPB associations should become involved in the goal of establishing training standards and credentials to ensure a high and consistent outcome. The ORANGE II trial in which techniques are standardised and a training and proctor programme is available, combined with the hybrid design of randomisation and registry may help to provide a framework for controlled and safe implementation of laparoscopic liver resection across participating centres. 


\title{
Conclusions
}

The international multicentre randomised controlled ORANGE II trial is based on the observations of more rapid recovery and discharge after laparoscopic liver resection, and more rapid recovery and discharge after open liver resection within an enhanced recovery programme. This is the first RCT to provide evidence on the merits of laparoscopic surgery in patients undergoing a LLS within an enhanced recovery programme.

\section{Trial status}

Currently there are nine actively participating centres, consisting of the Maastricht University Medical Centre (MUMC), Academic Medical Centre (AMC), University Medical Centre Utrecht (UMCU), Erasmus Medical Centre (Erasmus MC), University Medical Centre Groningen (UMCG), Maxima Medical Centre (MMC), Medical Spectrum Twente (MST) in The Netherlands. In addition, the University Hospital Aachen (UK Aachen) and San Raffaele Hospital Milan (HSR Milan) are two participating centres in Germany and Italy. Additional centres will be invited to participate.

\begin{abstract}
Abbreviations
ORANGE, Optimised recovery with accelerated nutrition and GI enhancement; ERAS, Enhanced recovery after surgery; LLS, Left lateral sectionectomy; LOS, Length of stay (in hospital); POD, Post-operative day; CRF, Case record form; ASA, American society of anaesthesiology; P-POSSUM, Portsmouth physiological and operative severity score for the enumeration of mortality and morbidity; EORTC, European organisation for research and treatment of cancer
\end{abstract}

\section{Competing interests}

Both positive and negative results will be published. There are no financial interests in this trial and none of the parties concerned has right of veto.

\section{Acknowledgements}

The ORANGE II trial is partly funded by the Profiling Fund of the Maastricht University Medical Centre, Maastricht, The Netherlands number PF 298. In addition, Covidien ${ }^{\circledR}$ supports this trial with an unrestricted grand.

Collaborators of the ORANGE II Study Group: O.R.C. Busch, P.J. Tanis, L.T. Hoekstra (Academic Centre Amsterdam), R. van Hillegersberg, I.Q. Molenaar (UMC Utrecht), C. Verhoef, T. Terkivatan, J. de Jonge (Erasmus MC), G.D. Slooter, R.M.H. Roumen 
(Maxima MC), J.M. Klaase, E.B. van Duyn (Medical Spectrum Twente), K. Boscha (Jeroen Bosch Hospital), R. J. Porte, M.T de Boer, J.W. Haveman (UMC Groningen), J.H.W. de Wilt, O.R. Buyne (UMC Nijmegen), P. van Duijvendijk (Gelre Hospitals), U. Neumann, M. Schmeding (UH Aachen), G. Ferla, L.A. Aldrighetti, F. Ferla (HSR MiIan), J.N. Primrose, M. Abu Hilal, N.W. Pearce (University Hospital Southampton), I. Dagher (Antoine Béclère Hospital), A. Laurent (Henri Mondor Hospital), B. Topal (UZ Leuven), R.I. Troisi (UZ Gent), B. Edwin (Oslo University Hospital).

DSMB members: M.A. Boermeester, I.H.M. Borel Rinkes, A.W. Ambergen.

\section{REFERENCES}

1. Ruers T, Bleichrodt RP. Treatment of liver metastases, an update on the possibilities and results. Eur J Cancer 2002; 38: 1023-1033.

2. Cuesta MA, Meijer S, Paul MA, et al. Limited laparoscopic liver resection of benign tumors guided by laparoscopic ultrasonography: report of two cases. Surg Laparosc Endosc 1995; 5: 396-401.

3. Carswell KA, Sagias FG, Murgatroyd B, et al. Laparoscopic versus open left lateral segmentectomy. BMC Surg 2009; 9(14): 9.

4. Vigano L, Tayar C, Laurent A, et al. Laparoscopic liver resection: a systematic review. J Hepatobiliary Pancreat Surg 2009; 16: 410-421.

5. Cherqui D. Laparoscopic liver resection. Br J Surg 2003; 90: 644-646.

6. Dagher I, Di Giuro G, Dubrez J, et al. Laparoscopic versus open right hepatectomy: a comparative study. Am J Surg 2009; 198: 173-177.

7. Dagher I, O'Rourke N, Geller DA, et al. Laparoscopic major hepatectomy: an evolution in standard of care. Ann Surg 2009; 250: 856-860.

8. Abu Hilal M, Di Fabio F, Teng MJ, et al. Single-centre comparative study of laparoscopic versus open right hepatectomy. J Gastrointest Surg 2011; 15(5): 818-823.

9. Abu Hilal M, McPhail MJ, Zeidan B, et al. Laparoscopic versus open left lateral hepatic sectionectomy: a comparative study. Eur J Surg Oncol 2008; 34: 1285-1288.

10. Abu Hilal M, Pearce NW. Laparoscopic left lateral liver sectionectomy: a safe, efficient, reproducible technique. Dig Surg 2008; 25: 305-308.

11. Dagher I, Lainas P, Carloni A, et al. Laparoscopic liver resection for hepatocellular carcinoma. Surg Endosc 2008; 22: 372-378.

12. Dagher I, Proske JM, Carloni A, et al. Laparoscopic liver resection: results for 70 patients. Surg Endosc 2007; 21: 619-624.

13. Lee KF, Cheung YS, Chong CN, et al. Laparoscopic versus open hepatectomy for liver tumours: a case control study. Hong Kong Med J 2007; 13: 442-448.

14. O'Rourke N, Shaw I, Nathanson L, et al. Laparoscopic resection of hepatic colorectal metastases. HPB (Oxford) 2004; 6: 230-235.

15. Troisi $R$, Montalti $R$, Smeets $P$, et al. The value of laparoscopic liver surgery for solid benign hepatic tumors. Surg Endosc 2008; 22: 38-44.

16. Nguyen KT, Gamblin TC, Geller DA. World review of laparoscopic liver resection-2,804 patients. Ann Surg 2009; 250: 831-841.

17. Bryant R, Laurent A, Tayar C, et al. Laparoscopic liver resection-understanding its role in current practice: the Henri Mondor Hospital experience. Ann Surg 2009; 250: 103-111.

18. Laurence JM, Lam VW, Langcake ME, et al. Laparoscopic hepatectomy, a systematic review. ANZ J Surg 2007; 77: 948-953. 
19. Alkari B, Owera A, Ammori BJ. Laparoscopic liver resection: preliminary results from a UK centre. Surg Endosc 2008; 22: 2201-2207.

20. Carswell KA, Sagias FG, Murgatroyd B, et al. Laparoscopic versus open left lateral segmentectomy. BMC Surg 2009; 9: 14.

21. Vigano L, Tayar C, Laurent A, et al. Laparoscopic liver resection: a systematic review. J Hepatobiliary Pancreat Surg 2009; 16: 410-421.

22. Fearon $\mathrm{KC}$, Ljungqvist $\mathrm{O}$, Von Meyenfeldt $\mathrm{M}$, et al. Enhanced recovery after surgery: a consensus review of clinical care for patients undergoing colonic resection. Clin Nutr 2005; 24: 466-477.

23. Kehlet $\mathrm{H}$, Wilmore DW. Multimodal strategies to improve surgical outcome. Am J Surg 2002; 183 : 630-641.

24. Wilmore DW, Kehlet $\mathrm{H}$. Management of patients in fast track surgery. BMJ (Clinical research ed 2001; 322: 473-476.

25. Lassen K, Soop M, Nygren J, et al. Consensus review of optimal perioperative care in colorectal surgery: enhanced recovery after surgery (ERAS) group recommendations. Arch Surg 2009; 144: 961-969.

26. Basse L, Madsen JL, Kehlet $\mathrm{H}$. Normal gastrointestinal transit after colonic resection using epidural analgesia, enforced oral nutrition and laxative. Br J Surg 2001; 88: 1498-1500.

27. Basse L, Raskov $\mathrm{HH}$, Hjort Jakobsen $\mathrm{D}$, et al. Accelerated postoperative recovery programme after colonic resection improves physical performance, pulmonary function and body composition. $\mathrm{Br} \mathrm{J}$ Surg 2002; 89: 446-453.

28. Delaney $\mathrm{CP}$, Zutshi $\mathrm{M}$, Senagore AJ, et al. Prospective, randomized, controlled trial between a pathway of controlled rehabilitation with early ambulation and diet and traditional postoperative care after laparotomy and intestinal resection. Dis Colon Rectum 2003; 46: 851-859.

29. Zutshi M, Delaney CP, Senagore AJ, et al. Randomized controlled trial comparing the controlled rehabilitation with early ambulation and diet pathway versus the controlled rehabilitation with early ambulation and diet with preemptive epidural anesthesia/analgesia after laparotomy and intestinal resection. Am J Surg 2005; 189: 268-272.

30. Van Dam RM, Hendry PO, Coolsen MM, et al. Initial experience with a multimodal enhanced recovery programme in patients undergoing liver resection. Br J Surg 2008; 95: 969-975.

31. Stoot JH, van Dam RM, Busch OR, et al. The effect of a multimodal fast-track programme on outcomes in laparoscopic liver surgery: a multicentre pilot study. HPB (Oxford) 2009; 11: 140-144.

32. Maessen J, Dejong $\mathrm{CH}$, Hausel J, et al. A protocol is not enough to implement an enhanced recovery programme for colorectal resection. Br J Surg 2007; 94: 224-231.

33. McCulloch P. Developing appropriate methodology for the study of surgical techniques. J R Soc Med 2009; 102: 51-55.

34. Gore SM. The consumer principle of randomisation. Lancet 1994; 343: 58.

35. Brewin CR, Bradley C. Patient preferences and randomised clinical trials. BMJ 1989; 299: 313-315.

36. Nguyen KT, Gamblin TC, Geller DA. World review of laparoscopic liver resection-2, 804 patients. Ann Surg 2009; 250: 831-841.

37. Dindo D, Demartines N, Clavien PA. Classification of surgical complications: a new proposal with evaluation in a cohort of 6336 patients and results of a survey. Ann Surg 2004; 240: 205-213.

38. Van den Broek MA, van Dam RM, van Breukelen GJ, et al. Development of a composite endpoint for randomized controlled trials in liver surgery. Br J Surg 2011; 98: 1138-1145.

39. Lamers LM, McDonnell J, Stalmeier PF, et al. The Dutch tariff: results and arguments for an effective design for national EQ-5D valuation studies. Health Econ 2006; 15: 1121-1132.

40. Lamers LM, Stalmeier PF, McDonnell J, et al. Measuring the quality of life in economic evaluations: the Dutch EQ-5D tariff. Ned Tijdschr Geneeskd 2005; 149: 1574-1578.

41. Langenhoff BS, Krabbe PF, Peerenboom L, et al. Quality of life after surgical treatment of colorectal liver metastases. Br J Surg 2006; 93: 1007-1014. 


\section{Chapter 15}

42. Blazeby JM, Fayers $\mathrm{P}$, Conroy $\mathrm{T}$, et al. Validation of the european organization for research and treatment of cancer QLQ-LMC21 questionnaire for assessment of patient-reported outcomes during treatment of colorectal liver metastases. Br J Surg 2009; 96: 291-298.

43. Polle SW, Dunker MS, Slors JF, et al. Body image, cosmesis, quality of life, and functional outcome of hand-assisted laparoscopic versus open restorative proctocolectomy: long-term results of a randomized trial. Surg Endosc 2007; 21: 1301-1307.

44. Dunker MS, Stiggelbout AM, van Hogezand RA, et al. Cosmesis and body image after laparoscopicassisted and open ileocolic resection for Crohn's disease. Surg Endosc 1998; 12: 1334-1340.

45. Oostenbrink JB, Koopmanschap MA, Rutten FF. Standardisation of costs: the Dutch manual for costing in economic evaluations. Pharmacoeconomics 2002; 20: 443-454.

46. Hoër J, Lawong $G$, Klinge $U$, et al. Einflussfaktoren der narbenhernienenstehung retrospektive untersuchung an 2.983 laparotomierten patienten über einen zeitraum vno 10 jahren. Chirurg 2002; 73: 474-480.

47. Andersen LP, Klein M, Gogenur I, et al. Incisional hernia after open versus laparoscopic sigmoid resection. Surg Endosc 2008; 22: 2026-2029.

48. D'Angelica M, Maddineni S, Fong $\mathrm{Y}$, et al. Optimal abdominal incision for partial hepatectomy: increased late complications with Mercedes-type incisions compared to extended right subcostal incisions. World J Surg 2006; 30: 410-418.

49. Togo $S$, Nagano $Y$, Masumoto $C$, et al. Outcome of and risk factors for incisional hernia after partial hepatectomy. J Gastrointest Surg 2008; 12: 1115-1120.

50. Paice JA, Cohen FL. Validity of a verbally administered numeric rating scale to measure cancer pain intensity. Cancer Nurs 1997; 20: 88-93.

51. McGuire DB. Comprehensive and multidimensional assessment and measurement of pain. J Pain Symptom Manage 1992; 7(5): 312-319.

52. Dorrepaal KL, Aaronson NK, van Dam FS. Pain experience and pain management among hospitalized cancer patients. A clinical study. Cancer 1989; 63: 593-598.

53. Jensen MP. The validity and reliability of pain measures in adults with cancer. J Pain 2003; 4(1): 221.

54. Krebs EE, Carey TS, Weinberger M. Accuracy of the pain numeric rating scale as a screening test in primary care. J Gen Intern Med 2007; 22: 1453-1458.

55. Suurmeijer TP, Doeglas DM, Moum T, et al. The Groningen Activity Restriction Scale for measuring disability: its utility in international comparisons. Am J Public Health 1994; 84: 1270-1273.

56. Snapinn SM. Monitoring clinical trials with a conditional probability stopping rule. Stat Med 1992; 11: 659-672.

57. Van Breukelen GJ, Candel MJ, Berger MP. Relative efficiency of unequal versus equal cluster sizes in cluster randomized and multicentre trials. Stat Med 2007; 26: 2589-2603.

58. Van den Broek MA, van Dam RM, Malago M, et al. Feasibility of randomized controlled trials in liver surgery using surgery-related mortality or morbidity as endpoint. Br J Surg 2009; 96: 10051014.

59. Koffron AJ, Auffenberg G, Kung R, et al. Evaluation of 300 minimally invasive liver resections at a single institution: less is more. Ann Surg 2007; 246: 385-392: discussion 392-384.

60. Vigano L, Laurent A, Tayar C, et al. The learning curve in laparoscopic liver resection: improved feasibility and reproducibility. Ann Surg 2009; 250: 772-782.

61. Buell JF, Cherqui D, Geller DA, et al. World Consensus Conference on Laparoscopic Surgery: The international position on laparoscopic liver surgery: the Louisville statement, 2008. Ann Surg 2009; 250: 6.

62. McCulloch P, Taylor I, Sasako M, et al. Randomised trials in surgery: problems and possible solutions. BMJ 2002; 324: 1448-1451.

63. Kazaryan AM, Pavlik Marangos I, Rosseland AR, et al. Laparoscopic liver resection for malignant and benign lesions: ten-year Norwegian single-centre experience. Arch Surg 145: 34-40. 
64. Topal B, Fieuws S, Aerts R, et al. Laparoscopic versus open liver resection of hepatic neoplasms: comparative analysis of short-term results. Surg Endosc 2008; 22: 2208-2213.

65. Pessaux $\mathrm{P}$, Regimbeau JM, Dondero $F$, et al. Randomized clinical trial evaluating the need for routine nasogastric decompression after elective hepatic resection. Br J Surg 2007; 94: 297-303.

66. Ito $\mathrm{K}$, Ito $\mathrm{H}$, Are $\mathrm{C}$, et al. Laparoscopic versus open liver resection: a matched-pair case control study. J Gastrointest Surg 2009; 13: 2276-2283. 



\section{Part 6}

General discussion 

Chapter 16

General discussion and summary 
The aim of the current thesis was to investigate ways to reduce the insult of surgical trauma in the treatment of liver tumours. These ways included reducing harm to patients by using novel treatment techniques for life threatening haemorrhaging of liver lesions, the application of minimally invasive liver surgery and the implementation of perioperative care protocols. Resection of part of the liver is nowadays a commonly performed procedure for a variety of malignant and benign hepatic tumours. ${ }^{1,2}$ Previous to the 1980 's, this type of surgery was still associated with a high morbidity and mortality. ${ }^{1,3,4}$ The perioperative outcome after hepatic resection has improved over time, due to increased knowledge of liver anatomy and function, improvement of operating techniques and advances in anaesthesia and post-operative care. ${ }^{2-4}$ In the first chapter a historical overview of the quest to improvement in liver surgery is described.

The first aim of this thesis was to analyse current treatments for echinococcosis of the liver and this is discussed in part 2.

The second aim of this thesis investigating improvements in liver surgery was to evaluate the management of ruptured adenomas of the liver with selective arterial embolisation. In addition, it was intended to discuss the need for resection of adenomas with the focus on the actual risk of malignant degeneration. This is discussed in part 3.

The third aim of this thesis on improving outcomes of liver surgery, was to focus on the two major developments in surgery: the minimal invasive techniques for resection of liver lesions and the multimodal perioperative care protocols such as the Enhanced Recovery After Surgery (ERAS) regimens. These two aspects of liver surgery are discussed in part 4. 


\section{Current treatments for echinococcosis of the liver}

The first aim of this thesis consists of analysing current treatments for Echinococcosis of the liver, a benign liver disease. In chapter $\mathbf{2}$, an unexpected encounter with the Echinococcus multilocularis in the liver, mimicking metastases of the liver is described. As far as we know, this represents the first patient of Echinococcus multilocularis acquired in the Netherlands. Having been shown to spread at a rate of 2.7 kilometres per year in a Northern direction due to a combination of an increase in foxes and an increase in their Echinococcus multilocularis load, ${ }^{5}$ Echinococcus multilocularis might become an important pathogen in Western Europe. Clinicians should consider this diagnosis when confronted with (asymptomatic) liver lesions.

In chapter 3, a single centre study is presented, which has been performed to evaluate the treatment and outcome of patients treated surgically for hydatid disease in a non-endemic area such as the Netherlands. In this retrospective study, 112 consecutive patients were treated surgically with the 'frozen seal' method for hydatid disease between 1981 and 2007. Recurrence rate was observed in 9 (8\%) patients and morbidity occurred in twenty patients (17.9\%). More importantly, in this study no mortality was seen in more than 25 years of surgically treated 'echinococcosis'. These results compare favourably with the current evidence even considering a recent meta-analysis of operative versus non-operative treatment (PAIR) of hepatic echinococcosis. ${ }^{6}$ In this meta-analysis, 14 studies were included concerning surgical treatment in endemic areas incorporating more than 950 patients. It showed an overall minor and major complication rate of $33 \%$ and $25 \%$ respectively. The mortality rate was nearly $1 \%$ among the surgically treated patients. These morbidity and mortality rates are comparable with other single centre studies with open surgical treatment. ${ }^{7}$

In conclusion, the - frozen seal - method of surgery from the past century is still safe and effective in the new millennium. This technique is especially useful in centres in non-endemic areas as it provides high efficacy and low morbidity rates. Future studies should prove its position in the treatment of hepatic hydatid disease as less invasive treatments such as PAIR show promising results.

In this single centre study, however, we also found a gradual decrease in the number of operations performed for echinococcosis. This could be caused by either a decrease in incidence of this rare disease or differences in treatment methods (for instance less invasive techniques or only medical therapy). In order to elucidate this finding, we investigated in chapter 4 the incidence of hepatic echinococcosis in the Netherlands and explored the opinions of experts concerning treatment of echinococcosis of the liver. Therefore, the national database of pathology (PALGA) was investigated and compared with results from serological testing from 1997 through 2008. In addition, to gain an overview of treatment regimes on hepatic echinococcosis, a questionnaire was sent to gastrointestinal surgeons in 20 hepato- 
pancreato-biliary centres in the Netherlands concerning incidence, diagnosis, therapy and follow-up. In this study, we found a decrease in the number of resected specimens (PALGA) from 2003 onwards. The incidence of echinococcosis based on serology also decreased from 2002 onwards to 33 patients per year. Seventeen (85\%) of twenty hospitals responded to the questionnaire of which seven (41\%) centres stated they had treated hydatid disease of the liver. Four $(57 \%)$ of these seven hospitals followed a protocol for the treatment of hydatid liver disease. Between these treating hospitals, primary treatment strategy varied considerably.

From this study on incidence, current opinions and treatment of hepatic echinococcosis in a country where this disease is non-endemic, the combined databases of pathology and serology indicate that the incidence of hydatid disease has decreased in the last decade. The actual incidence is low and therefore hydatid disease remains rare. Remarkably, the primary treatments differ between specialised centres, and a guideline might improve the uniformity in treatment strategies. In the Netherlands, there are only two large centres dealing with this disease. Therefore, regionalization of this rare disease might improve the results as proven in other surgical treatments. Only then, can centres increase their expertise and a gradual shift to less invasive and safer treatment is to be expected. Management of this disease should be multidisciplinary, stage specific and custom made.

\section{Evaluation of management of ruptured adenomas of the liver with selective arterial embolisation}

Management of hepatocellular adenomas is still open to debate. In a highly selected group, namely pregnant patients with pre-eclampsia an increased risk of tumour rupture exists. In this chapter we focus on the rupture of adenomas in pregnancy describing a patient with a life threatening condition of liver rupture after labour. The management is discussed in chapter 5 .

A primi gravida at term was diagnosed as having pre-eclampsia, and she remained hemodynamically unstable after delivery. Further analysis with a contrast enhanced CT-scan revealed four lesions in the liver resembling adenomas and free peritoneal fluid. Because the patient could not be stabilized with conservative treatment, selective embolisation of the right hepatic artery was performed successfully. Haemorrhage in pregnancy from ruptured liver adenomas is rare and therefore easily misdiagnosed. Presentation may be with severe pain localised to the epigastrium or right upper quadrant of the abdomen with radiation to the back or right shoulder. These symptoms may be misinterpreted as dyspepsia or cholecystitis. Delay in diagnosing rupture of the adenomas will contribute to high mortality rates. It is essential diagnosis is made at once, preferably using contrast enhanced CT-scanning. When a ruptured adenoma is discovered during pregnancy, 
selective arterial embolisation is a suitable treatment in order to avoid invasive surgery.

In chapter 6 the management of partial hepatectomy after successful initial treatment of selective arterial embolisation is described. Since partial hepatectomy is generally recommended in patients with lesions $\geq 5 \mathrm{~cm}$, because of increased risk of rupture as well as malignant degeneration, ${ }^{8,9}$ resection was carried out several months after successful selective arterial embolisation. Interestingly, the tumour consisted of necrotic material and no specific histological tumour classification could be made. This might be due to the embolisation, which caused cessation of arterial blood flow resulting in necrosis of the tumour. This might correspond with the fact that hepatocellular adenomas are vascularised arterially. The necrosis of the tumour may point to a potential future role for selective arterial embolisation in the elective management of hepatocellular adenomas. This would further limit the indications for surgery in a selected group of patients, resulting in reduction of patient morbidity and mortality.

Controversy remains concerning the emergency treatment of patients with a ruptured hepatocellular adenoma, as some authors still advise acute surgical resection as the primary treatment, ${ }^{10,11}$ despite an associated mortality rate up to $8 \% .{ }^{12}$ In order to refrain from major liver surgery to avoid this risk, especially in an acute setting, selective arterial embolisation is a novel method for the management of intra-abdominal haemorrhage from hepatocellular adenomas. In contrast, some authors have suggested a more conservative approach with primarily hemodynamical stabilization, followed by preventive partial liver resection for tumours exceeding five $\mathrm{cm}$ because of potential malignant degeneration. ${ }^{8,13}$

In chapter $\mathbf{7}$ it is shown that selective arterial embolisation for ruptured and haemorrhaged hepatocellular adenomas is a safe and effective method to stabilise hemodynamically unstable patients. None of the eleven consecutive patients required surgery and only one required additional embolisations. There were a few minor complications consisting of fever, probably caused by tissue necrosis after embolisation. In the follow-up period of 19 months all adenomas including those without signs of haemorrhaging in the same liver lobe $(n=25)$ were either smaller or not detectable on follow-up CT or MRI scans. This decrease in size of all adenomas was statistically significant.

In conclusion, selective artery embolisation is a valuable tool in the acute management of haemorrhaging hepatic adenomas and should be considered as the first choice in treatment. In this way, emergency resection can be avoided and a reduction in morbidity and mortality is to be expected. If no secondary partial hepatectomy is planned, long-term follow-up of these patients is needed. Therefore, preferably MRI scanning should be used to discriminate between residual haematoma, cyst formation, and residual liver tumour. Increase in tumour size might necessitate 
the need for a more aggressive approach including biopsy, ablation or surgical resection.

In chapter $\mathbf{8}$ a systematic review of the literature is presented with a focus on the risk of malignant transformation of hepatocellular adenomas(HCA) into hepatocellular carcinomas(HCC). The study shows that malignant alteration is a rare complication of these uncommon benign tumours. By means of a systematic search of studies reporting cases of this benign liver disease over the past 40 years and combining the data on these HCAs, a total of 1568 reported HCAs were found. The overall frequency of malignant transformation reported was $4.2 \%$ among all adenoma cases and $4.5 \%$ among all resected HCAs.

These results identify a small but serious risk of malignant transformation of hepatocellular adenomas. It is important to recognize factors that increase the risk of malignant transformation, or even better, identify factors that can more easily discriminate between these two entities as both have similar imaging characteristics and histopathological features. Tumour size greater than $5 \mathrm{~cm}$ is currently regarded as a risk factor. The recommendation however, to only resect HCAs larger than $5 \mathrm{~cm}$ in diameter in order to prevent malignant transformation is open for discussion since three cases have been reported in which malignant transformation occurred in a tumour measuring less than $5 \mathrm{~cm}$ in diameter. ${ }^{14-16}$

Another proposed risk factor for malignant alteration is the presence of dysplasia in HCAs. This characteristic harbours a risk of progression to HCC. ${ }^{17-21}$ The Bordeaux group ${ }^{22,23}$ has performed genotype-phenotype analyses in HCAs and found markers that are a promising method of identifying these high-risk adenomas. They identified four different tumour subtypes with specific characteristics: (1) hepatocyte nuclear factor $1 \alpha$ (HNF1 $\alpha$ ) mutated (30\%-50\%), (2) $\beta$-catenin-activated (10$15 \%),(3)$ inflammatory (35\%) and (4) unclassified tumours (5\%-10\%) $)^{22-24}$ Hepatocellular carcinoma associated with adenoma was found in $46 \%$ of $\beta$-catenin-mutated tumours, whereas this has never been observed in inflammatory lesions and rarely found in HNF1 $\alpha$-mutated tumours. It was concluded that HCA's with $\beta$-catenin activation have a higher risk of malignant transformation.

For all patients presenting one or more HCA's larger than $5 \mathrm{~cm}$, resection is the preferred treatment in accordance with the current literature. If however, only $4.2 \%$ of HCA's have actual foci of HCC, many resections will be performed while there is no risk of malignant degeneration. It is concluded that more research is needed to investigate the mechanism of malignant degeneration. Only then can this group of predominantly young patients be withheld a potentially unnecessary liver resection as this surgical treatment still has a morbidity and mortality rate of up to $27 \%$ and $3 \%$ respectively. ${ }^{2,3,25,26}$ Promising methods to identify these high-risk adenomas are the Bordeaux HCA markers.

In conclusion, the current systematic review (chapter 8) shows that malignant transformation of hepatocellular adenomas into hepatocellular carcinomas is a rare 
complication of these uncommon benign tumours. By careful selection for surgical treatment of only those patients with hepatocellular adenomas harbouring an increased risk of malignant alteration, fewer patients will be subjected to unnecessary surgery. This would reduce the risks associated with surgery in these predominantly young patients.

\section{The effect of the minimal invasive techniques for resection of liver lesions and ERAS on the outcome of liver surgery}

In part 4, the first focus was on the introduction of a new surgical approach for liver resections: the minimally invasive or laparoscopic technique. Furthermore, the effect of implementation of a multimodal perioperative programme and laparoscopic liver surgery was also investigated. The initial results of this new approach of resectional liver surgery were evaluated in the Netherlands.

Although many expert centres perform laparoscopic liver surgery, conventional liver surgeons need to clear some hurdles before they feel comfortable operating on the liver from a certain distance using a laparoscope. Another drawback of this technique might be the high costs. Managers and Operation Room (OR) personnel are not aware of the beneficial effects for patients and might just look at their own budget and time.

The introduction of this new technique in Maastricht University Medical Centre (MUMC) showed that the costs of laparoscopic liver resections were to equivalent to those of open surgery (chapter 9). Furthermore, implementation of a laparoscopic liver resection programme seems feasible and safe with reduced blood loss and operation time but comparable morbidity and length of stay. It is interesting to observe that there were no significant differences in the length of hospital stay between the open and laparoscopic approach. Striking is the relatively long stay in hospital for the laparoscopic procedures. We found no valid explanation for this. When introducing such new techniques, they are predominantly started in young and healthy patients with benign lesions. One might postulate that it is surgery that makes these young healthy women into patients, as they had hardly any complaints before surgery. Open surgery is performed in patients with malignancies who feel cured after surgery. The relatively long hospital stay was previously also observed for colonic resections before and after the start of a perioperative multimodal fast track setting (Enhanced Recovery After Surgery; ERAS ${ }^{\circledR}$ ). ${ }^{27}$ Although in this recent study predefined discharge criteria were assessed, almost $90 \%$ of the ERAS patients did not return home on the day the discharge criteria were actually met. After careful investigation of valid reasons for prolonged stay, $69 \%$ of extended hospital stays were labelled as inappropriate. Only a minority was rated as medically necessary. It is remarkable that even with preoperative counselling in the ERAS programme, a difference between functional recovery and actual day of dis- 
charge is observed. This implies that the difference in length of stay may be even more pronounced if all patients were to return home once they are functionally recovered according to predefined criteria. ${ }^{27,28}$

In order to improve the care of the surgical patient with liver lesions, it is clear we must to implement the evidence available. It takes many presentations, publications and programmes to change daily practice. In many ways, the ERAS principle may serve as an example for this, the subject of which is described in part 4. ERAS, the multimodal enhanced recovery programme, has already been adopted by many centres throughout the world and is currently becoming standard care. The beneficial effects have been proven by many publications in colorectal and liver surgery and include less pain, earlier functional recovery and shorter hospital length of stay. This multimodal recovery programme is evidence based and combines several interventions in perioperative care to reduce the stress response and organ dysfunction with a focus on enhancing recovery. However, even in a small country such as the Netherlands not all hospitals adhere to this protocol.

This therefore provided the ideal situation to evaluate the additional value of an ERAS programme for laparoscopic liver resections for solid tumours, as described in chapter 10. A group of patients undergoing laparoscopic liver resections in an ERAS setting in the Maastricht was compared with historical data from consecutive laparoscopic liver resections performed either in that same centre before the introduction of the ERAS programme or in other major liver centres in the Netherlands performing laparoscopic liver surgery in a traditional perioperative care programme.

In chapter 10, the data suggests that a multimodal enhanced recovery programme for laparoscopic liver surgery is feasible and safe. A significant difference in the median time to full functional recovery of two days was observed between the ERAS treated group and the traditional care group. The difference in median LOS of two days between these two groups did not attain significance, likely due to the small number of patients in this study. Apart from quick functional recovery in patients in the enhanced recovery group, this study also showed reduced blood loss in this group. As the ERAS protocol prescribes a low CVP and the avoidance of excessive perioperative fluids, it may be caused by the implementation of this protocol. However, this difference in blood loss might also be clouded by the learning curve effect, since all patients operated in the traditional care were the first patients to be treated laparoscopically in the participating centres.

In conclusion, this exploratory multicentre fast track laparoscopic liver resection study is the first such study conducted. Although small, the study suggests that a multimodal enhanced recovery programme for laparoscopic liver surgery is feasible, safe and may lead to accelerated functional recovery and a reduction in the length of hospital stay. With these findings it may be concluded that the additional effect of ERAS leads to an improvement of liver surgery and outcome. However, 
functional recovery may be a better instrument to investigate differences in outcome of surgical treatments in future studies. It will be a challenge to investigate the effect of ERAS programmes on laparoscopic liver surgery in a randomised controlled setting as proposed in chapter 15.

In chapter 11, we aimed to investigate the initial experience with laparoscopic liver resections in the Netherlands with a focus on factors influencing hospital length of stay. For this purpose, all thirty patients undergoing laparoscopic left lateral sectionectomy (Couinaud segments 2 and 3 ) in the Netherlands in the years 2000 to 2008 were included and compared with a group of ninety patients undergoing the same type of liver resection as an open procedure in the same era. This multicentre study demonstrated that length of hospital stay for the laparoscopic approach for left lateral sectionectomies was reduced by two days compared to the open procedure. Furthermore, it was found that the laparoscopic approach resulted in faster procedures with reduced blood loss and comparable morbidity. In a multivariable regression model, ASA classification, complications and ERAS proved to be important prognostic variables for reduced length of hospital stay.

As stipulated earlier in this thesis, HPB surgeons will have to decide with the lack of clear evidence: either to adopt this new technique with evidence available from expert centres or, as suggested by others, ${ }^{29,30}$ to perform multicentre randomised trials to obtain the highest level of evidence. Currently, new initiatives (ORANGE II - Trial) have been launched by the Maastricht University Medical Centre HPB surgical unit in collaboration with the Dutch Working group for Liver Surgery.

Laparoscopic surgery and enhanced recovery programmes have been shown to improve outcome by enhancing recovery and reducing hospital length of stay. Laparoscopic surgery is a challenging surgical technique and is often introduced without clear evidence to support its superiority compared to open surgery. This is in contrast with the adoption of perioperative care protocols, which are not implemented easily worldwide, but are indeed evidence based. A survey among the international HPB community was undertaken to clarify the difference in adoption of these two surgical developments. This survey showed that the majority of HPB centres performed liver surgery in the absence of an enhanced recovery perioperative care programme, and performed laparoscopic liver surgery, although the laparoscopic volumes were low. As both were associated with faster recovery, it was concluded that fast-track programmes should receive more attention. Furthermore, the need for a randomised trial was confirmed by this recent survey among the international liver surgical community (chapter 12) 


\section{FUTURE PERSPECTIVES}

As more major liver resections are performed, our aim in chapter $\mathbf{1 3}$ was to compare two open source image processing software packages to measure prospectively the remnant liver volume in order to reduce the risk of post-resectional liver failure. Of the 15 patients included, volumes of total liver, tumour and future resection specimen were measured preoperatively with ImageJ and OsiriX by two surgeons and a surgical trainee. Results were compared with the actual weights of resected specimens and the measurements of the radiologist using professional CT scanner-linked Aquarius iNtuition ${ }^{\circledR}$ software. We found that the prospective hepatic CT-volumetry with ImageJ or Osiri ${ }^{\circledR}$ was reliable and can be accurately used on a Personal Computer by non-radiologists. ImageJ and OsiriX ${ }^{\circledR}$ yielded results comparable to professional radiological software iNtuition ${ }^{\circledR}$.

In chapter $\mathbf{7}$ we showed that embolising ruptured adenomas prevented growth of these lesions. Subsequently, selective arterial embolisation was utilised in a number of non-haemorrhaging adenomas. ${ }^{31}$ During follow-up, none of these adenomas grew and the majority even regressed in size. Upon separate examination of the haemorrhaging and non-haemorrhaging adenomas, a statistically significant decrease in size was noted in both groups. It is this tumour regression and its probable subsequent reduction of the risk of severe haemorrhaging and malignant transformation that might support selective arterial embolisation as a novel treatment for large unruptured HCAs. As no significant complications from this treatment were reported arterial embolisation of HCAs might be the direction for further future research. ${ }^{31,32}$

In chapter 14 a randomised controlled trial with a two-arm experimental design is proposed to investigate this new approach in the treatment paradigm of hepatocellular adenomas.

The aim is to test the hypothesis that minimally invasive embolisation of nonruptured HCAs leads to a reduction of the post-treatment morbidity and mortality (i.e. any event) compared with a resectional approach. Following a protected fine needle biopsy, patients with a negative $\beta$-catenin status who are eligible for resection of their HCA will be randomised to either resection or selective arterial embolisation. The primary endpoint is the proportion of patients with treatment-related morbidity and mortality (within 90 days after treatment). Secondary outcome parameters include functional recovery, quality of life and post-treatment bodyimage changes as well as direct and indirect medical costs. It is expected that this trial will provide evidence on the merits of selective arterial embolization of HCA.

Lastly, in chapter $\mathbf{1 5}$ an international multicentre randomized controlled trial is proposed which is expected to provide level 1 scientific evidence on the effects of ERAS protocols and laparoscopic versus open liver surgery. It is an international multicentre randomized controlled trial based on the observations of a faster re- 
covery and discharge after laparoscopic liver resection as well as a faster recovery and discharge after open liver resection within an enhanced recovery programme. We expect that this trial will provide evidence on the merits of laparoscopic surgery in patients undergoing a left lateral hepatic sectionectomy within an enhanced recovery programme.

\section{REFERENCES}

1. Cescon M, Vetrone G, Grazi GL, Ramacciato G, Ercolani G, Ravaioli M, et al. Trends in perioperative outcome after hepatic resection: analysis of 1500 consecutive unselected cases over 20 years. Ann Surg. 2009;249(6):995-1002.

2. Poon RT, Fan ST, Lo CM, Liu CL, Lam CM, Yuen WK, et al. Improving perioperative outcome expands the role of hepatectomy in management of benign and malignant hepatobiliary diseases: analysis of 1222 consecutive patients from a prospective database. Ann Surg. 2004;240(4):698-708.

3. Jarnagin WR, Gonen M, Fong $Y$, DeMatteo RP, Ben-Porat L, Little S, et al. Improvement in perioperative outcome after hepatic resection: analysis of 1,803 consecutive cases over the past decade. Ann Surg. 2002;236(4):397-406.

4. Tsao JI, Loftus JP, Nagorney DM, Adson MA, Ilstrup DM. Trends in morbidity and mortality of hepatic resection for malignancy. A matched comparative analysis. Ann Surg. 1994;220(2):199-205.

5. Takumi K, de Vries A, Chu ML, Mulder J, Teunis P, van der Giessen J. Evidence for an increasing presence of Echinococcus multilocularis in foxes in The Netherlands. Int J Parasitol. 2008;38(5):5718.

6. Smego RA, Jr., Bhatti S, Khaliq AA, Beg MA. Percutaneous aspiration-injection-reaspiration drainage plus albendazole or mebendazole for hepatic cystic echinococcosis: a meta-analysis. Clin Infect Dis. 2003;37(8):1073-83.

7. Yagci G, Ustunsoz B, Kaymakcioglu N, Bozlar U, Gorgulu S, Simsek A, et al. Results of surgical, laparoscopic, and percutaneous treatment for hydatid disease of the liver: 10 years experience with 355 patients. World J Surg. 2005;29(12):1670-9.

8. Terkivatan T, de Wilt JH, de Man RA, van Rijn RR, Tilanus HW, JN IJzermans. Treatment of ruptured hepatocellular adenoma. Br J Surg. 2001;88(2):207-9.

9. Ault GT, Wren SM, Ralls PW, Reynolds TB, Stain SC. Selective management of hepatic adenomas. Am Surg. 1996;62(10):825-9.

10. Cobey FC, Salem RR. A review of liver masses in pregnancy and a proposed algorithm for their diagnosis and management. Am J Surg. 2004;187(2):181-91.

11. Cho SW, Marsh JW, Steel J, Holloway SE, Heckman JT, Ochoa ER, et al. Surgical management of hepatocellular adenoma: take it or leave it? Ann Surg Oncol. 2008;15(10):2795-803.

12. Eckhauser FE, Knol JA, Raper SE, Thompson NW. Enucleation combined with hepatic vascular exclusion is a safe and effective alternative to hepatic resection for liver cell adenoma. Am Surg. 1994;60(7):466-71.

13. Terkivatan T, de Wilt JH, de Man RA, ljzermans JN. Management of hepatocellular adenoma during pregnancy. Liver. 2000;20(2):186-7.

14. Micchelli ST, Vivekanandan P, Boitnott JK, Pawlik TM, Choti MA, Torbenson M. Malignant transformation of hepatic adenomas. Mod Pathol. 2008;21(4):491-7.

15. Dokmak S, Paradis V, Vilgrain V, Sauvanet A, Farges O, Valla D, et al. A Single Centre Surgical Experience of 122 Patients with Single and Multiple Hepatocellular Adenomas. Gastroenterology. 2009;137(5):1698-705.

16. Kim DH, Kim SU, Nam DH, Choi YJ, Park SM, Lee CK, et al. A case of hepatocellular carcinoma within hepatocellular adenoma in a non-cirrhotic male. Korean J Intern Med. 2009;24(2):147-52. 


\section{Chapter 16}

17. Anthony PP, Vogel CL, Barker LF. Liver cell dysplasia: a premalignant condition. J Clin Pathol. 1973;26(3):217-23.

18. Ho JC, Wu PC, Mak TK. Liver cell dysplasia in association with hepatocellular carcinoma, cirrhosis and hepatitis B surface antigen in Hong Kong. Int J Cancer. 1981;28(5):571-4.

19. Lee RG, Tsamandas AC, Demetris AJ. Large cell change (liver cell dysplasia) and hepatocellular carcinoma in cirrhosis: matched case-control study, pathological analysis, and pathogenetic hypothesis. Hepatology. 1997;26(6):1415-22.

20. Su Q, Benner A, Hofmann WJ, Otto G, Pichlmayr R, Bannasch P. Human hepatic preneoplasia: phenotypes and proliferation kinetics of foci and nodules of altered hepatocytes and their relationship to liver cell dysplasia. Virchows Arch. 1997;431(6):391-406.

21. Tao LC. Oral contraceptive-associated liver cell adenoma and hepatocellular carcinoma. Cytomorphology and mechanism of malignant transformation. Cancer. 1991;68(2):341-7.

22. Zucman-Rossi J, Jeannot E, Nhieu JT, Scoazec JY, Guettier C, Rebouissou S, et al. Genotypephenotype correlation in hepatocellular adenoma: new classification and relationship with HCC. Hepatology. 2006;43(3):515-24.

23. Bioulac-Sage P, Blanc JF, Rebouissou S, Balabaud C, Zucman-Rossi J. Genotype phenotype classification of hepatocellular adenoma. World J Gastroenterol. 2007;13(19):2649-54.

24. Bluteau $\mathrm{O}$, Jeannot $\mathrm{E}$, Bioulac-Sage $\mathrm{P}$, Marques JM, Blanc JF, Bui $\mathrm{H}$, et al. Bi-allelic inactivation of TCF1 in hepatic adenomas. Nat Genet. 2002;32(2):312-5.

25. Charny CK, Jarnagin WR, Schwartz LH, Frommeyer HS, DeMatteo RP, Fong Y, et al. Management of 155 patients with benign liver tumours. Br J Surg. 2001;88(6):808-13.

26. Terkivatan $\mathrm{T}$, de Wilt JH, de Man RA, van Rijn RR, Zondervan PE, Tilanus HW, et al. Indications and long-term outcome of treatment for benign hepatic tumors: a critical appraisal. Arch Surg. 2001;136(9):1033-8.

27. Maessen JM, Dejong $\mathrm{CH}$, Kessels AG, von Meyenfeldt MF. Length of stay: an inappropriate readout of the success of enhanced recovery programmes. World J Surg. 2008;32(6):971-5.

28. Maessen J, Dejong CH, Hausel J, Nygren J, Lassen K, Andersen J, et al. A protocol is not enough to implement an enhanced recovery programme for colorectal resection. Br J Surg. 2007;94(2):22431.

29. Simillis C, Constantinides VA, Tekkis PP, Darzi A, Lovegrove R, Jiao L, et al. Laparoscopic versus open hepatic resections for benign and malignant neoplasms--a meta-analysis. Surgery. 2007;141(2):203-11.

30. Mala T, Edwin B. Role and limitations of laparoscopic liver resection of colorectal metastases. Dig Dis. 2005;23(2):142-50.

31. Stoot JH, van der Linden E, Terpstra OT, Schaapherder AF. Life-saving therapy for haemorrhaging liver adenomas using selective arterial embolization. Br J Surg. 2007;94(10):1249-53.

32. Erdogan D, Busch OR, van Delden OM, Ten Kate FJ, Gouma DJ, van Gulik TM. Management of spontaneous haemorrhage and rupture of hepatocellular adenomas. A single centre experience. Liver Int. 2006;26(4):433-8. 


\section{Hoofdstuk 17}

\section{Nederlandse samenvatting}




\section{INLEIDING}

In dit proefschrift wordt de verbetering van chirurgische zorg bij patiënten met een leverafwijking behandeld. De doelstellingen waren de huidige behandelingen van zeldzame afwijkingen van de lever te evalueren, nieuwe technieken te onderzoeken voor de behandeling van levensbedreigende bloedingen van leverafwijkingen en minimaal invasieve ofwel laparoscopische chirurgie te onderzoeken in combinatie met peri-operatieve zorgprotocollen. Dit proefschrift kent drie thema's: Chirurgische behandeling van echinokokkose (infectie van de lever), embolisatie van leveradenomen (goedaardige levertumoren) en laparoscopische leverchirurgie. Het huidige hoofdstuk is een samenvatting van elk van de voorafgaande hoofdstukken.

\section{HISTORISCH OVERZICHT}

Het eerste hoofdstuk beschrijft een historisch overzicht van de zoektocht naar verbetering van leveroperaties. De behandeling van levertumoren heeft de laatste decennia een enorme vlucht genomen. Het operatief verwijderen ofwel reseceren van een deel van de lever is tegenwoordig een gebruikelijke procedure voor verschillende goedaardige (benigne) en kwaadaardige (maligne) levertumoren. In de tachtiger jaren van de vorige eeuw ging deze ingreep nog gepaard met een hoog ziekte- en sterftecijfer (morbiditeit en mortaliteit). Inmiddels is het resultaat na een leverresectie verbeterd dankzij de toegenomen kennis van de leveranatomie en functie, de verbetering van operatietechnieken en de verbeteringen in de anesthesie en post-operatieve zorg.

\section{CHIRURGISCHE BEHANDELING VAN ECHINOKOKKOSE}

\section{Echinokokkose van de lever}

In hoofdstuk 2 wordt de behandeling beschreven van een goedaardige leverziekte 'Echinococcus multilocularis' (echinokokkose van de lever), die gelijkenis vertoont met uitzaaiingen in de lever. Voor zover wij weten werd in deze studie de eerste patiënt onderzocht die Echinococcus multilocularis heeft opgelopen in Nederland. Artsen dienen deze diagnose te overwegen indien ze geconfronteerd worden met (asymptomatische) leverafwijkingen. 


\section{Chirurgische behandeling van de echinokokkose in de lever}

In hoofdstuk 3 wordt een studie gepresenteerd van de chirurgische behandeling van echinokokkose van de lever in een niet-endemisch ${ }^{1}$ gebied als Nederland. In deze retrospectieve studie ${ }^{2}$ werden 112 opeenvolgende patiënten onderzocht die chirurgisch werden behandeld tussen 1981 en 2007 met de 'frozen seal'-methode ${ }^{3}$ (zie figuur 3, hoofdstuk 1). De ziekte keerde terug ofwel recidiveerde bij 9 patiënten $(8 \%)$ en 20 patiënten (18\%) werden complicaties (morbiditeit) vastgesteld, zonder dat er sterfte was ten gevolge van deze behandeling. Dit toont aan dat de 'frozen seal'-methode nog steeds veilig en effectief is in het nieuwe millennium. Echter, toekomstige studies zullen deze chirurgische techniek van echinokokkose van de lever verder moeten beoordelen, nu minder invasieve technieken veelbelovende resultaten laten zien.

\section{Nederlandse studie naar behandeling van echinokokkose.}

In de studie beschreven in hoofdstuk 3 vonden wij ook een geleidelijke afname van het aantal operaties voor echinokokkose. Dit kan worden veroorzaakt door een daling van de incidentie van deze zeldzame ziekte of door verschillen in behandelingsmethoden (bijvoorbeeld minder invasieve technieken of andere medicinale therapie). Om deze bevinding nader te bestuderen, onderzochten we in hoofdstuk 4 de incidentie van echinokokkose in Nederland en enquêteerden wij deskundigen over de behandeling hiervan. Daarvoor werden alle rapporten onderzocht van aangeprikte en chirurgisch verwijderde weefsels met echinokokkose vanuit de nationale database van pathologie (PALGA). De uitkomsten werden vergeleken met de resultaten van serologisch onderzoek van 1997 tot 2008. Tot slot, om een overzicht van de behandelingen van echinokokkose te krijgen, werd een vragenlijst gestuurd naar chirurgen in 20 levercentra in Nederland met betrekking tot incidentie, diagnose, therapie en follow-up. Deze gegevens uit de gecombineerde databases van pathologie en serologie tonen aan dat de incidentie van echinokokkose is afgenomen in Nederland in de laatste 10 jaar. Opmerkelijk is dat de initiële behandelingen in de gespecialiseerde centra verschillen. De werkelijke incidentie is laag en daarom blijft deze ziekte nog steeds zeldzaam.

\footnotetext{
${ }^{1}$ niet inheems

${ }^{2}$ met reeds verzamelde gegevens

${ }^{3}$ Soort open kegel die wordt vastgevroren aan het leveroppervlak tijdens de chirurgische ingreep.
} 


\section{EMBOLISATIE VAN LEVERADENOMEN}

\section{Evaluatie van de behandeling van leveradenomen}

Het beleid bij goedaardige levertumoren (leveradenomen) staat nog steeds ter discussie. In een zeer selecte groep, namelijk zwangere vrouwen met pre-eclampsie (zwangerschapsvergiftiging), is er een verhoogd risico op scheuren (ruptureren) van deze levertumoren. In hoofdstuk 5 beschrijven wij een patiënte met een levensbedreigende leverruptuur na de bevalling. Bloeding van gescheurde leveradenomen in de zwangerschap is zeldzaam, hetgeen de kans op een verkeerde diagnose vergroot. Ernstige pijn gelokaliseerd boven in de buik of in het rechter bovenkwadrant met uitstraling naar de rug of rechterschouder kan verkeerd worden geïnterpreteerd als dyspepsie (spijsverteringklachten) of cholecystitis (galblaasontsteking). Vertraging bij het stellen van de diagnose van gescheurde leveradenomen zal bijdragen tot hoge mortaliteit. Het is daarom essentieel om de diagnose direct in het kraambed te stellen, bij voorkeur door middel van een CT-scan. Wanneer de diagnose van een gescheurde leveradenoom bij een zwangere is gesteld, is selectieve embolisatie (bloedvatafsluiting) een geschikte behandeling.

\section{Chirurgie na selectieve arteriële embolisatie}

In hoofdstuk 6 wordt een leverresectie (verwijdering van een deel van de lever) beschreven na een succesvolle eerste behandeling met selectieve arteriële embolisatie $^{4}$ van een bloedend adenoom. Met deze techniek wordt via een catheter in de lies, de slagader naar de tumor afgesloten. Over het algemeen wordt aanbevolen om bij patiënten met afwijkingen $\geq 5 \mathrm{~cm}$ deze tumor te verwijderen vanwege een verhoogd risico op scheuren en/of maligne (kwaadaardige) ontaarding. Enkele maanden na een succesvolle selectieve arteriële embolisatie, is een resectie uitgevoerd. Het bleek dat de tumor bestond uit necrotisch (dood) weefsel bij de beschreven patiënte en er geen specifieke histologische diagnose ${ }^{5}$ kon worden gesteld. Dit zou passen bij het feit dat deze adenomen via de arterie (slagader) van bloed worden voorzien. De necrose van de tumor maakt een toekomstige rol voor selectieve arteriële embolisatie in de behandeling van leveradenomen mogelijk. Dit zou in een geselecteerde groep patiënten verdere inperking van de indicatie voor chirurgie betekenen, hetgeen leidt tot vermindering van morbiditeit en mortaliteit. $\mathrm{Er}$ is nog steeds controverse over de acute behandeling van patiënten met gescheurde leveradenomen. Sommige auteurs adviseren acuut chirurgisch ingrijpen met resectie als primaire behandeling, ondanks een bijbehorend sterftecijfer tot

\footnotetext{
${ }^{4}$ afsluiting van de slagader

${ }^{5}$ van het weefsel
} 
8\%. Om dit sterfterisico te verlagen, in het bijzonder in een acute setting, is selectieve arteriële embolisatie een nieuwe methode voor de behandeling van intraabdominale ${ }^{6}$ bloedingen van leveradenomen. Andere auteurs echter, stellen een meer conservatieve aanpak voor met in de eerste plaats bloeddruk (hemodynamische) stabilisatie, later gevolgd door preventieve partiële leverresectie voor tumoren van meer dan $5 \mathrm{~cm}$ vanwege mogelijk risico op maligne ontaarding.

\section{De acute behandeling met selectieve arteriële embolisatie}

In hoofdstuk 7 wordt aangetoond dat selectieve arteriële embolisatie voor gescheurde en bloedende leveradenomen een veilige en effectieve methode is om hemodynamisch onstabiele patiënten te stabiliseren. Bij geen van de 11 opeenvolgende patiënten was chirurgisch ingrijpen noodzakelijk en slechts een enkele patient moest een extra embolisatie-behandeling ondergaan. In de controleperiode na de behandeling waren alle adenomen kleiner geworden, ook degene die geen tekenen van bloedingen hadden vertoond. Sommige waren zelfs niet meer aantoonbaar op de CT-of MRI-scans. Geconcludeerd wordt dat selectieve arteriële embolisatie een waardevolle behandeling is bij acute bloedingen van leveradenomen en dat deze techniek als de eerste keuze beschouwd moet worden. Hierdoor kan een acute operatie worden vermeden en is een vermindering in de morbiditeit en mortaliteit te verwachten. Wanneer er geen secundaire leveroperatie volgt, is lange termijn controle van deze patiënten nodig.

\section{Onderzoek van alle gerapporteerde leveradenomen in de literatuur}

In hoofdstuk 8 wordt een systematische review van de literatuur gepresenteerd met een focus op het risico van maligne ontaarding van goedaardige leveradenomen in kwaadaardige levertumoren (carcinomen). Op basis van systematisch studieonderzoek naar deze goedaardige leverziekte in de afgelopen 40 jaar zijn 1.568 gemelde leveradenomen gevonden. De totale frequentie van maligne ontaarding was $4,2 \%$ onder alle leveradenomen en $4,5 \%$ onder alle geopereerde leveradenomen. Uit dit onderzoek blijkt dat de kwaadaardige verandering een zeldzame complicatie is van deze goedaardige tumoren. In de literatuur blijft de aanbeveling om leveradenomen groter dan $5 \mathrm{~cm}$ te opereren. Echter, indien slechts bij $4,2 \%$ van alle leveradenomen kwaadaardigheid wordt gevonden, zullen vele operaties onnodig worden verricht. Er zal meer onderzoek nodig zijn om het mechanisme van maligne ontaarding te onderzoeken. Alleen dan kan deze groep van voornamelijk jonge patiënten een mogelijk onnodige leverresectie worden bespaard. Deze chirurgische behandeling heeft immers nog steeds een morbiditeit van $27 \%$ en mortaliteit van

\footnotetext{
${ }^{6}$ in de buikholte
} 
3\%. Veelbelovende methoden om de hoog-risico adenomen te identificeren, zijn de Bordeaux leveradenomen markers, waarvan $\beta$-catenine de belangrijkste is. Door een zorgvuldige selectie voor chirurgische behandeling van alleen die patiënten met leveradenomen met een verhoogd risico voor maligne ontaarding, zullen minder patiënten onnodige leveroperaties ondergaan.

\section{LAPAROSCOPISCHE LEVERCHIRURGIE}

\section{Evaluatie invoering laparoscopische leverchirurgie}

In deel 4 van dit proefschrift, beginnend met hoofdstuk 9, ligt de nadruk op de invoering van een nieuwe chirurgische aanpak voor leverresecties: de minimaal invasieve ofwel laparoscopische techniek. Ook is het effect onderzocht van laparoscopische leverchirurgie en van een multimodaal peri-operatief programma ${ }^{7}$ dat is uitgevoerd. De eerste resultaten van deze nieuwe techniek voor leveroperaties in Nederland zijn geëvalueerd. Hoewel veel expertcentra laparoscopische leverchirurgie uitvoeren, moeten conventionele leverchirurgen een aantal hindernissen nemen voordat ze deze nieuwe operatietechniek beheersen. Met de laparoscoop wordt vanaf grotere afstand, met minder weefselgevoel en twee dimensionaal geopereerd. Een ander nadeel van deze techniek zouden de hoge kosten kunnen zijn. Echter, de introductie van deze nieuwe techniek in Maastricht Universitair Medisch Centrum (MUMC) liet zien dat de kosten van laparoscopische leverresecties gelijk waren aan open operaties. Daarnaast bleek dat de implementatie van een laparoscopisch leverresectie-programma goed uitvoerbaar en veilig was, met beperkt bloedverlies en operatietijd. De morbiditeit en duur van het verblijf in het ziekenhuis verschilden niet.

\section{Enhanced Recovery after Surgery (ERAS) ofwel versneld herstelprogramma}

ERAS, een multimodaal herstelprogramma ter bespoediging van het patiëntherstel na chirurgie, is reeds goedgekeurd door vele centra over de hele wereld en is momenteel steeds meer de standaardzorg. De gunstige effecten ervan zijn bewezen in vele publicaties over darm- en leverchirurgie: er is eerder functioneel herstel en een kortere ziekenhuisopname. Dit multimodale herstelprogramma is evidence based $^{8}$ en combineert een aantal interventies in de peri-operatieve zorg om de stressrespons en orgaanfalen te verminderen met het doel het herstel te verbeteren. Desondanks is zelfs in een klein land als Nederland dit protocol niet in alle

\footnotetext{
${ }^{7}$ programma voor, tijdens en na de operatie bestaande uit meerdere aspecten die herstel bevorderen

${ }^{8}$ gebaseerd op onderzoek / wetenschappelijk bewijs
} 
ziekenhuizen ingevoerd. Hierdoor kon de toegevoegde waarde van een ERASprogramma voor laparoscopische leverresecties bij solide tumoren in Nederland worden geëvalueerd. In hoofdstuk 10 zijn twee groepen patiënten vergeleken: een groep patiënten dat laparoscopische leveroperaties onderging in ERAS-setting en een groep patiënten dat een laparoscopische leveroperatie onderging zonder ERAS-programma maar met traditionele nazorg. Dit multicentre, laparoscopische leverresectie-onderzoek naar versneld herstel is de eerste studie van dergelijke opzet.

De resultaten tonen aan dat een multimodaal herstelprogramma voor laparoscopische leverchirurgie goed werkt en een gelijk aantal complicaties heeft. Tussen de ERAS behandelde groep en de traditioneel behandelde groep werd een verschil van twee dagen waargenomen in functioneel herstel. Het verschil van twee dagen in hospitalisatieduur tussen deze twee groepen was echter niet statistisch significant. Dit is waarschijnlijk te wijten aan het kleine aantal patiënten in deze studie. Desondanks suggereert deze studie dat een multimodaal versneld herstelprogramma (ERAS) voor laparoscopische leverchirurgie haalbaar en veilig is en kan leiden tot versneld functioneel herstel en vermindering van de duur van verblijf in het ziekenhuis. Met deze resultaten kan worden geconcludeerd dat het aanvullende ERAS-effect bij de laparoscopische operatietechniek kan leiden tot een verbetering van het resultaat van leverchirurgie. Het is een uitdaging om het effect van ERAS-programma's op laparoscopische leveroperaties te onderzoeken in een gerandomiseerde gecontroleerde studieopzet, zoals voorgesteld in hoofdstuk 15.

\section{Laparoscopische leverresecties in Nederland}

In hoofdstuk 11 worden de eerste ervaringen met laparoscopische leverresecties in Nederland beschreven met de nadruk op factoren die van invloed zijn op de duur van het ziekenhuisverblijf. Voor dit doel werden alle 30 patiënten die laparoscopische linker laterale segment resectie ${ }^{9}$ ondergingen in Nederland in de jaren 2000 tot en met 2008 vergeleken met een groep van 90 patiënten in dezelfde periode die een open procedure ondergingen voor hetzelfde type leverresectie. Deze multicentre studie toont aan dat de verblijfsduur in het ziekenhuis voor de laparoscopische benadering voor de linker laterale leveroperatie werd verlaagd met twee dagen in vergelijking tot de open procedure. Bovendien werd vastgesteld dat de laparoscopische benadering resulteerde in snellere operaties met minder bloedverlies en vergelijkbare morbiditeit. In een multivariaat regressiemodel ${ }^{10}$ bleken ASA

\footnotetext{
${ }^{9}$ verwijdering van de linker (laterale) leverkwab

${ }^{10}$ een statistische berekenmethode
} 
classificatie $^{11}$, complicaties en ERAS belangrijke prognostische variabelen voor een kortere verblijfduur in het ziekenhuis.

\section{Internationale enquête}

Met laparoscopische chirurgie en verbeterde herstelprogramma's is aangetoond dat het herstel versneld en het ziekenhuisverblijf verkort kan worden. Hoofdstuk 12 omschrijft laparoscopische chirurgie als een uitdagende chirurgische techniek, die vaak wordt ingevoerd zonder duidelijk bewijs ter ondersteuning van haar superioriteit in vergelijking met een open operatie. Dit in tegenstelling tot de perioperatieve zorgprotocollen, die niet gemakkelijk over de hele wereld zijn toegepast maar wel evidence based zijn. In het kader van dit proefschrift werd een enquête uitgevoerd onder de internationale leverchirurgen om het verschil van deze twee chirurgische ontwikkelingen te verduidelijken. De meerderheid van de levercentra werkte zonder een verbeterd peri-operatief zorgprogramma maar voerde wel laparoscopische leveroperaties uit, hoewel de aantallen per centrum laag waren. Aangezien beide werden geassocieerd met een sneller herstel, werd geconcludeerd dat versneld herstelprogramma's meer aandacht moeten krijgen. Bovendien werd de noodzaak van een gerandomiseerde studie bevestigd door deze recente enquête onder de internationale leverchirurgische gemeenschap.

\section{TOEKOMSTPERSPECTIEVEN}

\section{Levervolumetrie}

Een ernstig risico van leveroperaties is leverfalen na de operatie. Dit kan voorkomen als een te groot gedeelte van de lever wordt weggehaald tijdens een operatie. Naarmate er meer grote leverresecties worden uitgevoerd, is het van belang om voor de operatie het te verwachten restant levervolume te meten om dit risico van leverfalen te verminderen. In hoofdstuk $\mathbf{1 3}$ wordt de vergelijking beschreven van twee 'open source software pakketten' ${ }^{12}$ die zijn gebruikt om prospectief ${ }^{13}$ het te verwachten restvolume van de lever te meten. Van 15 opgenomen patiënten werden volumes van de totale lever, de tumor en het toekomst resectiepreparaat preoperatief gemeten met ImageJ en OsiriX ${ }^{\circledR}$ door twee chirurgen en een onderzoeker. De resultaten werden vergeleken met het werkelijke gewicht van de verwijderde weefsels en de afmetingen van de radioloog met de professionele CT-

\footnotetext{
${ }^{11}$ de ASA (American Society of Anesthesiologists): klassering van te opereren patiënten volgens ernst van voorafbestaande ziekte/orgaanlijden.

${ }^{12}$ beschikbare software, in dit geval ImageJ en OsiriX ${ }^{\circledR}$.

${ }^{13}$ Voor de operatie
} 
scanner en de hieraan gekoppelde software iNtuition ${ }^{\circledR}$. Wij vonden dat de lever-CTvolumetrie met ImageJ of OsiriX ${ }^{\circledR}$ betrouwbaar was en nauwkeurig kon worden gebruikt op een Personal Computer door niet-radiologen. Het ImageJ en OsiriX ${ }^{\circledR}$ resultaat is vergelijkbaar met de professionele radiologische software iNtuition ${ }^{\circledR}$.

\section{'SILVER'trial: Selectieve arterlële emboLisatie VErsus Resectie van leveradenomen}

In hoofdstuk 7 hebben wij laten zien dat embolisatie van geruptureerde adenomen latere groei van deze afwijkingen kan voorkomen. Ook werd selectieve arteriële embolisatie gebruikt in een aantal niet-bloedende adenomen. Gedurende de controleperiode hierna groeide geen van deze adenomen en toonde het merendeel zelfs regressie. Bij het afzonderlijk onderzoeken van grootte van bloedende en nietbloedende leveradenomen, werd een statistisch significante verkleining vastgesteld in beide groepen. Het is vanwege deze tumorregressie en de te verwachten verdere verlaging van het risico op ernstige bloedingen en kwaadaardige ontaarding, dat selectieve arteriële embolisatie als een nieuwe behandeling voor grote niet geruptureerde leveradenomen aantrekkelijk is. Aangezien er geen statistisch significante complicaties van deze behandeling werden gemeld, kan arteriële embolisatie van leveradenomen de richting zijn voor verder toekomstig onderzoek. In hoofdstuk 14 wordt een gerandomiseerde gecontroleerde trial voorgesteld met een twee-armig experimenteel ontwerp om deze nieuwe aanpak te onderzoeken bij de behandeling van leveradenomen. Het doel is nagaan in hoeverre minimaal invasieve embolisatie bij niet-geruptureerde leveradenomen leidt tot een vermindering van de morbiditeit en mortaliteit vergeleken met een operatieve benadering. $\mathrm{Na}$ een beschermde, fijne naald biopsie, zullen patiënten met een negatieve $\beta$-cateninestatus ('laag risico adenomen'), die in aanmerking komen voor resectie van hun leveradenoom, worden gerandomiseerd voor ofwel resectie of selectieve arteriële embolisatie. Het primaire eindpunt is de met behandeling gerelateerde morbiditeit en mortaliteit (binnen 90 dagen na de behandeling). Secundaire uitkomsten zijn: functioneel herstel, kwaliteit van leven en, na de behandeling, veranderingen in lichaamsbeeldperceptie, naast directe en indirecte medische kosten. De verwachting is dat deze studie gegevens zal opleveren over de voordelen van selectieve arteriële embolisatie van leveradenomen.

\section{ORANGE II trial: Laparoscopische versus open leverresecties met ERAS programma}

Ten slotte wordt in hoofdstuk 15 een tweede trial voorgesteld om de effecten van ERAS-protocollen bij laparoscopische en open leveroperaties te evalueren. Het is een internationale, multicentrisch, gerandomiseerde studie gebaseerd op de waarnemingen van een sneller herstel en ontslag na laparoscopische leverresectie, 
evenals een sneller herstel en ontslag na een open leverresectie met een ERASherstelprogramma. Ook hier verwachten wij dat deze studie gegevens zal opleveren over de voordelen van laparoscopische chirurgie bij patiënten die een leveroperatie ondergaan binnen een versneld herstelprogramma.

De hoofdstukken 16 en 17 behoeven geen nadere toelichting, daar het de general discussion is in het Engels (hoofdstuk 16) en bovenstaande Nederlandse samenvatting daarvan (hoofdstuk 17). 


\section{Acknowledgements}

All, who contributed to this thesis 'Improving the outcome of liver surgery' have my sincere gratefulness for their collaboration. Although this thesis bears yet one name, it would not have been possible with the help and support of various individuals. Being aware of the danger that exists by listing names of people who directly or indirectly contributed to this work, I have no doubt unintentionally overlooked important contributors, therefore I apologize beforehand.

I would like to express my appreciation to all colleagues and personnel of the Surgery Department in Leiden University Medical Centre, Maastricht University Medical Centre, Haga Hospital formerly known as Red Cross Hospital, Orbis Medical Centre and Atrium Medical Centre. Furthermore, I would like to thank my colleagues of the Dutch Liver Collaborative Group for their collaboration.

Especially, I would like to thank the following people to whom I am indebted for reasons best known to themselves: Lee Bouwman, Marieke de Boer, Paul Breslau, Olivier Busch, Mariel Casparie, Chivo's of MUMC (2007-2009), Robert-Jan Coelen, Ronald van Dam, Kees Dejong, Mechteld Dejong, Simon Dello, Laura van Dommelen, Bas Havekes, Tineke Herremans, Richard van Hillegersberg, Volkert Huurman, Kees Jongsma, Titia Kortbeek, lone Limantoro, Toine Lodewick, Micha Luyer, Steven Olde Damink, Residents of Atrium MC, Maastricht UMC, Orbis MC and last but definitely not least Red Cross Hospital (2003-2006), Sandro Schaapherder, Hans Schipper, Meindert Sosef, Jan Stoot Sr., Onno Terpstra, Ruben Visschers, Jaco Verweij, Bjorn Winkens, Edgar Wong-Lun-Hing, Members of Maatschap Heelkunde Zuid-Limburg.

I am grateful for the continuous love and support of my parents and family, not only during this PhD-project but also for the last 39 years. I would like to thank Philip, Anthony and Emilie for realising my dream to live an extremely happy life with lots of fun. Finally and most importantly I would like to thank Patricia for all her love, understanding and encouragement without which I would not have accomplished what I have today. 



\section{Curriculum Vitae}

The author was born on December $29^{\text {th }}$ in 1972 in Heerlen, the Netherlands. After having graduated from the Bernardinus College in 1991, he started the study of Medicine at the University of Leiden in 1992. During the interim year as he awaited admittance to study Medicine due to the Numerus Fixus system, he completed his first year study of Law. Having successfully passed his medical masters degree in 1997, he moved to California to work as a visiting researcher at Stanford University at the Department of Cardiothoracic Surgery (Prof. B.A. Reitz). Here, he developed his research and surgical skills under guidance of Prof. R.C. Robbins and dr. P.W.H.E. Vriens, with whom he worked on several transplantation projects. In 1998, he returned to the Netherlands to start his clinical internships in combination with studying Law on health care. After obtaining his Medical Practitioner Degree and American Medical Exam part II, he started surgical training in the Leiden University Medical Centre in 2001 (Prof.dr. O.T. Terpstra). He continued training in the Hagahospital, formerly known as the Red Cross Hospital (dr. P.J. Breslau, dr. H. Boutkan). During his residency, he studied acute treatment of life threatening condition of ruptured liver adenomas. This study received the Schoemaker Award in 2007 of the Associations of Surgeons of the Netherlands.

In 2006, the opportunity raised to continue his career in Maastricht, where he finished his residency in General Surgery (Prof.dr. J.W. Greve) and started his advanced training in Gastro-Intestinal Surgery (CHIVO) in Maastricht University Medical Centre (MUMC) (Prof.dr. C.H.C. Dejong). In the MUMC, he gained further experience in advanced abdominal surgery, especially the laparoscopic approach for gastrointestinal and liver surgery. Here he started other research projects. His main interest was treatment of benign liver lesions and improving outcome by means of laparoscopic liver surgery. Furthermore, the perioperative care improvements made by the multimodal Enhanced Recovery After Surgery (ERAS) programmes was one of his interests. He visited the HPB Surgery and Transplantation Department of the Royal Infirmary Edinburgh, Schotland, (Prof. O.J. Garden) by means of the Dutch Association of Gastro-Intestinal Surgery Exchange fellowship in 2007.

Since 2009 the author is a consultant gastrointestinal and oncological surgeon in the Orbis Medical Centre in Sittard. In 2011 the department of surgery of the Orbis Medical Centre merged with the Atrium Medical Centre in Heerlen. As of this date his consultancy expanded to Heerlen. Currently he lives in Maastricht with his loving wife Patricia and their wonderful kids: Philip, Anthony and Emilie. 



\section{List of publications}

Open versus laparoscopic left lateral hepatic sectionectomy within an enhanced recovery ERAS(R) programme (ORANGE II - Trial): study protocol for a randomized controlled trial. van Dam RM, Wong-Lun-Hing EM, van Breukelen GJ, Stoot JHMB, van der Vorst JR, Bemelmans MH, OldeDamink SW, Lassen K, Dejong CH, Study Group OI.

Trials. 2012 May 6;13:54

Laparoscopic liver resection in the Netherlands: how far are we? Stoot JHMB, Wong-Lun-Hing EM, Limantoro I, Visschers R, Busch OR, Van Hillegersberg R, De Jong KM, Rijken AM, Kazemier G, Olde Damink SW, Lodewick TM, Bemelmans MH, van Dam RM, Dejong $\mathrm{CH}$.

Dig Surg. 2012;29(1):70-8

The introduction of a laparoscopic liver surgery programme: a cost analysis of initial experience in a University hospital. Stoot JHMB, van Dam RM, Coelen RJ, Winkens $\mathrm{B}$, Olde Damink SW, Bemelmans $\mathrm{MH}$, Dejong $\mathrm{CH}$.

Scand J Surg. 2012;101(1):32-7.

The First Locally Acquired Human Infection of Echinococcus multilocularis in The Netherlands. van Dommelen L, Stoot JHMB, Cappendijk VC, Abdul Hamid MA, Stelma FF, Kortbeek LM, van der Giessen J, Oude Lashof AM.

J Clin Microbiol. 2012;50(5):1818-20.

Gut and liver handling of interleukin-6 during liver resection in man. Dello SA, Bloemen JG, van de Poll MC, van Dam RM, Stoot JHMB, van den Broek MA, Buurman WA, Bemelmans MH, Olde Damink SW, Dejong $\mathrm{CH}$.

HPB (Oxford). 2011 May;13(5):324-31.

A rare cause of obstructive jaundice and weight loss in Von Recklinghausen's disease. Bukkems SF, Stoot JHMB, Driessen A, Beets-Tan RG, van Nieuwenhoven MA, Masclee AM.

Neth J Med. 2010 Dec;68(12):414-7 
Malignant transformation of hepatocellular adenomas into hepatocellular carcinomas: a systematic review including more than 1600 adenoma cases. Stoot JHMB, Coelen RJS, de Jong MC, Dejong CHC HPB (Oxford) 2010; 12(8): 509-522.

Prospective Volumetric Assessment of the Liver on a Personal Computer by Nonradiologists Prior to Partial Hepatectomy. Dello SAWG, Stoot JHMB, van Stiphout RSA, Bloemen JG, Wigmore SJ, Dejong CHC, van Dam RM.

World J Surg. 2011; 35(2): 386-392.

More than 25 years of surgical treatment of hydatid cysts in a nonendemic area using the "frozen seal" method. Stoot JHMB, Jongsma CK, Limantoro I, Terpstra OT, Breslau PJ.

World J Surg. 2010 Jan;34(1):106-13.

A man with chyloperitoneum: a case report. van Dijk WA, Stoot JHMB.

Acta Chir Belg. 2009;109(5):651.

The effect of a multimodal 'fast track' programme on laparoscopic liver surgery - a multicentre pilot study. Stoot JHMB, Van Dam RM, Busch ORC, Van Hillegersberg R, De Boer M, Olde Damink SWM, Bemelmans MHA, Dejong CHC.

HPB 2009; 11: 140-144

Inflammatory aneurysm of the common iliac artery mimicking appendicitis. Mieog JS, Stoot JHMB, Bosch JJ, Koning OH, Hamming JF.

Vascular. 2008;16(2):116-9.

Symptomatic abdominal aorta aneurysm with inferior vena cava malposition. Evers D, Stoot JHMB, Breslau PJ.

Acta chir belg, 2007; 107, 693-694

Primary retroperitoneal mucinous cystadenoma with mural 'sarcoma like' nodule: A case report and review of the literature. Bakker RFR, Stoot JHMB, Blok P, Merkus JW. Virchows Arch. 2007;451(4):853-7

Life saving therapy for haemorrhaging liver adenomas using selective arterial embolisation. Stoot JHMB, van der Linden E, Terpstra OT, Schaapherder AFM. BJS, 2007; 94:1249-53.

Een kind met geprononceerde schedelnaden. Stoot JHMB, van Meurs AFM.

NTvG, 2007;151:2325 
Life threatening haemorrhage from adenomas in the liver during pregnancy. Stoot JHMB, Van Roosmalen J, Terpstra OT, Schaapherder AFM.

Dig Surg 2006;23:155-155

Necrosis of a large hepatic tumor after hemorrhage and subsequent selective arterial embolization. Huurman VAL, Stoot JHMB, Van Der Linden E, Terpstra OT, Schaapherder AFM.

World J Gastroenterol, 2006;12[37], 6059-6061.

Pre-transplant blood transfusion and cyclosporine $A$ induce long-term hamster cardiac xenograft survival in immunocompetent rats. Vriens PW, Stoot JHMB, van der Steenhoven TJ, Hoyt G, Bouwman E, Robbins RC.

Xenotransplantation 2005;12:63-71

Pre-transplant blood transfusion induces tolerance to hamster cardiac xenografts in athymic nude rats. Vriens PW, Stoot JHMB, Hoyt G, Scheringa M, Bouwman E, Robbins RC.

Xenotransplantation. 2001 Nov;8(4):247-57.

Xeno-tolerance induced by blood transfusion in athymic nude rats. Vriens PW, Stoot JHMB, Bouwman E, Robbins RC.

Transplant Proc. 2001;33(1-2):791

Blood transfusion effect on xenograft survival in athymic nude rats is mediated by humoral factors. Vriens PW, Stoot JHMB, Bouwman E, Robbins RC.

Transplant Proc. 2000 Aug;32(5):1034.

Radionuclide imaging of acute lung transplant rejection with Annexin V. Blankenberg FG, Robbins RC, Stoot JHMB, Vriens PW, Berry GJ, Tait JF, Straus HW.

Chest, 2000; 117:834-840

${ }^{18}$ F-FDG et physiopathologie tumorale. Stoot JHMB, Ribeiro MJS, McCready VR, Van Santen WHC, Bourguignon M, Mazière B, Pauwels EKJ.

Médecine Nucléaire, 1999, 23; 4: 239-247

The mechanism of accumulation of tumour-localising radiopharmaceuticals. Pauwels EKJ, McCready V.R., Stoot JHMB, Van Deurzen DFP.

Eur J Nucl Med 1998, 25: 277-305 
The use of ${ }^{99 m}$ Technetium labeled annexin $V$ for in vivo imaging of apoptosis during cardiac allograft rejection. Vriens PW, Blankenberg FG, Stoot JHMB, Ohtsuki K, Berry GJ, Tait JF, Strauss HW, Robbins RC.

J Thorac Cardiovasc Surg 1998; 116: 844-853

FDG Accumulation and tumor biology. Pauwels EKJ, Ribeiro MJ, Stoot JHMB, McCready VR, Bourguignon $M$, Mazière $B$.

Nucl Med Biol, 1998, 25; 4: 317-322.

\section{Collaborator}

A conservative and minimally invasive approach to necrotizing pancreatitis improves outcome. van Santvoort HC, Bakker OJ, Bollen TL, Besselink MG, Ahmed Ali $\mathrm{U}$, Schrijver AM, Boermeester MA, van Goor $\mathrm{H}$, Dejong $\mathrm{CH}$, van Eijck $\mathrm{CH}$, van Ramshorst B, Schaapherder AF, van der Harst E, Hofker S, Nieuwenhuijs VB, Brink MA, Kruyt PM, Manusama ER, van der Schelling GP, Karsten T, Hesselink EJ, van Laarhoven CJ, Rosman C, Bosscha K, de Wit RJ, Houdijk AP, Cuesta MA, Wahab PJ, Gooszen HG; Dutch Pancreatitis Study Group.

Gastroenterology. 2011;141(4):1254-63.

A step-up approach or open necrosectomy for necrotizing pancreatitis. van Santvoort HC, Besselink MG, Bakker OJ, Hofker HS, Boermeester MA, Dejong CH, van Goor $\mathrm{H}$, Schaapherder AF, van Eijck $\mathrm{CH}$, Bollen TL, van Ramshorst B, Nieuwenhuijs VB, Timmer R, Laméris JS, Kruyt PM, Manusama ER, van der Harst E, van der Schelling GP, Karsten T, Hesselink EJ, van Laarhoven CJ, Rosman C, Bosscha K, de Wit RJ, Houdijk AP, van Leeuwen MS, Buskens E, Gooszen HG; Dutch Pancreatitis Study Group.

N Engl J Med. 2010;362(16):1491-502. 